\title{
ChEMISORPTION aND CATALYTIC REACTIVITY OF COBALT AND SULFUR MONOLAYERS ON ORDERED MOLYBDENUM SURFACES
}

\author{
DISSERTATION \\ Submitted in partial satisfaction of the requirements \\ for the degree of \\ DOCTOR OF PHLISOPHY \\ in Chemistry \\ in the graduate division of the \\ University of California at Berkeley \\ Colette C. Knight \\ Materials Sciences Division \\ Center for Advanced Materials \\ Lawrence Berkeley Laboratory \\ University of Califomia \\ Berkeley, CA 94720 U.S.A.
}




\title{
Chemisorption and Catalytic Reactivity of Cobalt and Sulfur Monolayers on Ordered Molybdenum Surfaces
}

\author{
by \\ Colette Clarissa Knight
}

\begin{abstract}
Complex cobalt / molybdenum sulfide catalysts are modelled by the chemisorption of cobalt and sulfur monolayers on the surfaces of molybdenum single crystals. The growth and structure of these overlayers on the flat (100), stepped (910) and (911) and kinked $(28,4,1)$ surfaces of molybdenum were investigated. The growth mode of cobalt overlayers on clean and sulfur covered molybdenum surfaces was characterized using Auger electron spectroscopy and $\mathrm{CO}$ chemisorption. These investigations reveal that cobalt first grows as a flat monolayer on clean molybdenum surfaces. Subsequently, cobalt multilayers form three dimensional islands. When cobalt is deposited on sulfur covered surfaces, the sulfur overlayer migrates to the top of the cobalt overlayer. This topmost sulfur overlayer significantly reduces the $\mathrm{CO}$ adsorption capacity of these surfaces relative to the clean and cobalt covered surfaces.

While the growth mode of cobalt overlayers are similar on the flat and stepped surfaces, we demonstrate that the number and type of ordered cobalt and sulfur structures that form on the flat and stepped surfaces are different. In the case of cobalt, an ordered (3X 1) structure is formed on the Mo(910) and $(28,4,1)$ surfaces. This surface structure does not develop on the clean (100) surface. Only one of the two possible $(3 \times 1)$ Co
\end{abstract}


domains are formed on the $M o(910)$ and $M o(28,4,1)$ surfaces. These domains have the one side of the $(3 \times 1)$ unit cell parallel to the step edges, suggesting that cobalt preferentially adsorbs at the step edges. The $(3 \times 1)$ structure does not form on the Mo(911) surface indicating that the step orientation can restrict the formation of certain ordered overlayers. For chemisorbed sulfur only a subset of the ordered overlayers that form on the flat $(100)$ surface nucleate on the $(910),(911)$ and $(28,4,1)$ surfaces. Ordered sulfur overlayers also show preferential formation of domains that maximize the number of sulfur-step atom bonds. The adsorption and ordering of sulfur overlayers on stepped and kinked molybdenum surfaces lead to a doubling of the step height and terrace width.

Thiophene hydrodiesulfurization (HDS) reactions were performed over molybdenum crystal surfaces modified by the chemisorption of sulfur, cobalt, carbon and sulfur plus cobalt. We found that the stepped and kinked molybdenum surfaces have reactivities up to a factor of two greater than the low Miller index (100) surface. The chemisorption of adsorbates decreased the thiophene HDS reactivity relative to the clean surfaces. Unlike the promotion seen by the addition of cobalt to industrial molybdenum sulfide catalysts, the deposition of cobalt on molybdenum single crystal surfaces did not lead to an increased hydrodesulfurization activity. The lack of enhanced reactivity suggests that this promotional effect is not associated with metallic cobalt and nolybienum. 
To My Parents,

You taught me that the world is my oyster, That whenever I struggle and persevere

I will eventually earn my pearl. 


\section{Table of Contents}

List of Figures . . . . . . . . . . . . . . . . . . . . . . vi

List of Tables . . . . . . . . . . . . . . . . . . xii

Acknowledgements . . . . . . . . . . . . . . . xiv

1 Is.troduction

1.1 General concepts of catalysis . . . . . . . . . . . . 1

1.2 A surface suience approach to catalysis . . . . . . . . . . 4

1.3 Hydrodesulfurization . . . . . . . . . . . . . 6

1.4 Promoter role of cobalt . . . . . . . . . . . . . . . 14

1.5 Molybdenum single crystals as model HDS catalysts: strengths and limitations . . . . . . . . . . . . . . 17

\section{Experimental}

2.1 Introduction . . . . . . . . . . . . . . . . . . . . .

2.2 Apparatus . . . . . . . . . . . . . . . 28

2.2.1 Sample manipulator . . . . . . . . . . . . . . 28

2.2.2 High pressure cell and reaction loop . . . . . . . . 29

2.2 .3 Pumps . . . . . . . . . . . . . . 32

2.2.4 Sulfur Source . . . . . . . . . . . . . 33

2.2.5 Cobalt source . . . . . . . . . . . . . . . . . .33

2.2.6 Retarding field analyzer . . . . . . . . . . . . . 35 
2.2.7 Mass spectrometer . . . . . . . . . . , 35

2.3 Surface analytical techniques

2.3.1 Auger electron spectroscopy . . . . . . . . . . 36

2.3.2 Low energy electron diffraction . . . . . . . . 42

2.3.3 Therrual desorption spectrometry . . . . . . . . . . . . . .

2.4 Sample preparation . . . . . . . . . . . . . . . . . 57

2.4.1 Preparation of single crystals . . . . . . . . . . . . . . . . . . .

2.4.2 Cystal cleaning procedures . . . . . . . . . 60

2.5 High pressure reactions . . . . . . . . . . . . . 60

3 Structures of the clean stepped and kimked Mo(100) surfaces

3.1 Introduction . . . . . . . . . . . . . . . . . . 65

3.2 Diffraction from stepped surfaces . . . . . . . . . . 66

3.3 Characterization of stepped and kinked Mo(100) surfaces . . . 74

3.4 Summary . . . . . . . . . . . . . . . 80

4 Growth of cobalt overlayers on clean, sulfur and carbon covered molybdenum surfaces

4.1 Introduction . . . . . . . . . . . . . . . . . . . . . .

4.2 Thermodynamic and kinetic influences on overlayer morphology . . 83

4.3 Determination of growth modes using Auger spectroscopy . . . 85

4.4 Determination of desorption energetics from thermal desorption experiments . . . . . . . . . . . . . . . 87

4.5 Growth of cobalt overlayers on clean molybdenum surfaces . . . 88

4.5.1 Auger studies . . . . . . . . . . . . . 88

4.5.2 Carbon monoxide desorption from cobalt overlayers on 


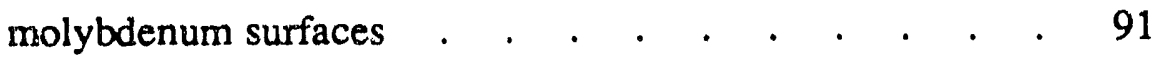

4.6 Growth of cobalt overlayers on adsorbate covered surfaces . . . 94

4.6.1 Auger studies . . . . . . . . . . . . . . . 94

4.6.2 Carbon monoxide thermal desorption . . . . . . 98

4.7 Desorption of cobalt overlayers from Mo(100) surfaces . . . . 100

4.8 Desorption of cobalt overlayers from $\mathrm{Mo}(28,4,1)$ and $\mathrm{Mo}(911)$ surfaces . . . . . . . . . . . . . . . 103

4.9 Summary . . . . . . . . . . . . . . . . . . 111

5 Structures of adsorbate overlayers on $\operatorname{Mo}(100)$, $M o(910)$,

$\operatorname{Mo}(911)$ and $M o(28,4,1)$ surfaces

5.1 Introduction . . . . . . . . . . . . . . . . 114

5.2 Diffraction from overlayers on vicinal surfaces . . . . . . 117

5.3 Sulfur overlayers on molybdenum surfaces $\quad . \quad$. . . . . . 121

5.4 Cobalt overlayers on molybdenum surfaces $\quad . \quad$. . . . . . $\quad .134$

5.5 Cobalt overlayers on sulfur covered molybdenum surfaces . . . 137

5.6 Summary . . . . . . . . . . . . . . . 144

6 Thiophene hydrodesulfurization over molybdenum surfaces

6.1 Introduction . . . . . . . . . . . . . . . 147

6.2 HDS over flat and vicinal molybdenum surfaces . . . . . 149

6.3 Thiophene HDS over sulfur and carbon covered molybdenum surfaces 154

6.4 Effect of cobalt on thiophene HDS over molybdenum surfaces . . 156

6.5 Summary . . . . . . . . . . . . . . . 161 


\section{List of Figures}

Figure 1.1: Top views of low Miller index surfaces of molybdenum. . . . . 3

Figure 1.2: Reaction for thiophene hydrodesulfurization over molybdenum single crystal surfaces。 . . . . . . . . . . . . . . . 9

Figure 1.3: Schematics of a) edge and basal bonding and b) structure of $\mathrm{MoS}_{2}$. .

Figure 1.4: The different cobalt phases coexisting on supported HDS catalysts. $\quad 12$

Figure 2.1: Spectrum of backscattered electrons from a solid after bombardment with $2 \mathrm{KeV}$ electrons. . . . . . . . . 24

Figure 2.2: Universal curve of electron mean free path in solids. . . . . 24

Figure 2.3: Photograph of ultra high vacuum system (taken in 1988). Since that time a new manipulator and cobalt source have added. . . . 27

Figure 2.4: Photograph of UHV / high pressure cell apparatus used in the experiments. The high pressure cell is shown in both the open (upper picture) and closed (lower picture) positions. . . . . . 28

Figure 2.5: Photographs of the sample holder. . . . . . . . . . 30

Figure 2.6: Schematic of high pressure cell and sealing surface of manipulator. . 31

Figure 2.7: Schematic of electrochernical sulfur source. . . . . . . . 34

Figure 2.8: Schematic of Auger process. . . . . . . . . . . . . . . . 38

Figure 2.9: Schematic of analyzer and signal amplification circuit used in the Auger experiment. . . . . . . . . . . . . . 40

Figure 2.10: Carbon Auger lineshapes for (a) a graphite overlayer on tungsten 
and $\mathrm{W}_{2} \mathrm{C}$ and (b) a carbidic overlayer on molybdenum. . . . . 41

Figure 2.11: Conservation of linear momentum parallel to the surface in a LEED experiment. . . . . . . . . . . . . . 44

Figure 2.12: Ewald sphere construction for low energy electron diffraction from surfaces. $k_{0}=$ initial wave vector, $k_{g}=$ final wave vector and $\mathrm{g}=$ any surface reciprocal vector. . . . . . . . . . 47

Figure 2.13: Schematic of LEED experiment. . . . . . . . . . . . 49

Figure 2.14: One-dimensional potential energy curves for (a) non activated dissociated adsorption through a physisorbed state and (b) activated chemisorption. . . . . . . . . . . . . . . . . . . . . . 52

Figure 2.15: Redhead's plot of first order activation energies of desorption as a function of the temperature at the maximum rate of desorption for heating rates $(\beta)$ of $1 \mathrm{~K} / \mathrm{s}, 20 \mathrm{~K} / \mathrm{s}, 50 \mathrm{~K} / \mathrm{s}$, $100 \mathrm{~K} / \mathrm{s}$ and $1000 \mathrm{~K} / \mathrm{s}$. . . . . . . . . . . . . 55

Figure 2.16: Schematic of thermal desorption experiment. . . . . . . . 56

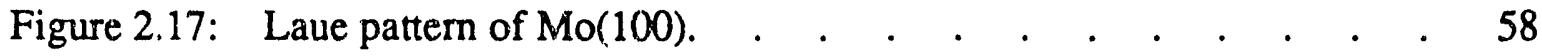

Figure 2.18: Gas chromatograph of compounds detected in thiophene HDS over Mo single crystal surfaces. . . . . . . . . . . . 62

Figure 3.1: Top views of (a) a low Miller index surface, (b) a corresponding stepped and (c) a corresponding kinked surface. The darker circles represent the step and kink atoms.

Figure 3.2: Schematics showing that a high Miller index surface with a given macroscopic orientation can have (a) monoatomic steps (b) diatomic steps or even (c) facets. . . . . . . . . . . . . . . . . 68

Figure 3.3: Constructive and destructive interference at a stepped surface. . . 70 
Figure 3.4: Ewald construction for stepped surfaces. . . . . . . . 71

Figure 3.5: Diffraction patterns of the clean vicinal surfaces. Schematic representations of the diffraction patterns are also shown.

On the schematics, the $(00),(01)$ and (10) beams, $\Delta \mathrm{K}_{01}$ and $\mathrm{K}_{01}$ are labelled. . . . . . . . . . . . . . 75

Figure 3.6: Diagrammatic representations of real-space (a) $\mathrm{Mo}(910)$, (b) Mo(28,4,1) and (c) Mo(911) surfaces. The upward arrow in (a) indicates both the [010] direction in the plane of the terrace and the [190] direction which is parallel to the macroscopic surface. The downward arrow in (b) indicates both the [010] direction in the plane of the terrace and the [170] direction which is parallel to the macroscopic surface. The diagonal arrow in (c) indicates both the [100] direction in the plane of the terrace and the [901] direction parallel to the macroscopic surface. . . . . . . . 79

Figure 4.1: Schematic representations of expected Auger plots of the three distinct growth modes: (a) Frank Van der Merwe, (b) Stranski-Krastanov and (c) Volmer-Weber growth modes. . . . 84

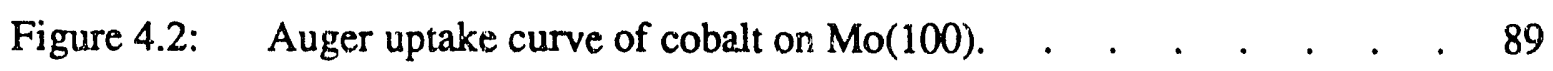

Figure 4.3: CO desorption from cobalt overlayers on $\mathrm{Mo}(28,4,1)$ surfaces. . . . 93

Figure 4.4: Plot of the intensity of the Mo (MNN) $186 \mathrm{eV}, \mathrm{S}(\mathrm{LMM}) 152 \mathrm{eV}$ and $\mathrm{Co}(\mathrm{LMM}) 775 \mathrm{eV}$ Auger transitions as a function of cobalt deposition time on the $M o(100)-c(2 \times 2) S$ surface. . . . . . 95

Figure 4.5: Auger spectra (a) before and (b) after air exposure of the $\mathrm{Co} / \mathrm{Mo}(100)-c(2 \times 2) \mathrm{S}$ surface. . . . . . . . . . . 97 
Figure 4.6: CO desorption from the following surfaces:

(a) $1.3 \mathrm{ML} \mathrm{Co} / \mathrm{Mo}(28,4,1)-\mathrm{c}(2 \times 2) \mathrm{S}$ (annealed at $870 \mathrm{~K})$,

(b) $1.3 \mathrm{ML} \mathrm{Co} / \mathrm{Mo}(28,4,1)-\mathrm{c}(2 \times 2) \mathrm{S}$ (unannealed),

(c) $\mathrm{Mo}(28,4,1)-\mathrm{c}(2 \times 2) \mathrm{S}$ and (d) $1.3 \mathrm{ML} \mathrm{Co} / \mathrm{Mo}(28,4,1)$.

99

Figure 4.7: Thermal desorption spectra of cobalt from (c) clean $\operatorname{Mo}(100)$ and

(b) $\mathrm{Mo}(100)-\mathrm{c}(2 \times 2) \mathrm{S}$ surfaces.

Figure 4.8: Plots of $\ln$ (desorption rate) against $1 / \mathrm{T}$ for the initial desorption

of cobalt multilayers on (a) clean $M o(100)$ and

(b) $\mathrm{Mo}(100)-c(2 \times 2) S$ surfaces. . . . . . . . . . . . 102

Figure 4.9: Cobalt desorption spectra from clean $\operatorname{Mo}(28,4,1)$ surfaces. . . 104

Figure 4.10: Cobalt desorption spectra from $\mathrm{Mo}(28,4,1)-(1 \times 1) \mathrm{C}$ surfaces. $\quad 105$

Figure 4.11: Cobalt desorption spectra from clean Mo(911) surfaces. . . . 106

Figure 4.12: Effect of pre-annealing on cobalt desorption from $\operatorname{Mo}(28,4,1)$ surfaces. . . . . . . . . . . . . . . . 108

Figure 4.13: Cobalt desorption from $\mathrm{Mo}(28,4,1)-\mathrm{c}(2 \times 2) \mathrm{S}$ surfaces. $\quad$. . 110

Figure 5.1: A $\quad(2 \times 2)$ overlayer on a square unit cell showing (a) correlations both perpendicular and parallel to the step edges and (b) only perpendicular correlations. In (c) there are no adsorbate correlations between adjacent terraces.

Figure 5.2: $\quad$ A $(2 \times 1)$ superlattice (large dots) on a square lattice (small dots) showing different (a) rotational domains and (b) translational domains.

Figure 5.3: Diffraction patterns and proposed real space models for (a) $c(2 \times 2),\left|\begin{array}{ll}2 & i \\ 1 & 1\end{array}\right|, c(4 \times 2)$ and $p(2 \times 1)$ sulfur overlayers on $\mathrm{Mo}(100)$ surfaces. 
Figure 5.4: Models of $\mathrm{Mo}(100)-\mathrm{p}(2 \times 1) \mathrm{S}$ surface showing the

(a) bridge-hollow and (b) asymmetric site models. . . . . . . 124

Figure 5.5: Diffraction patterns of $c(2 \times 2) S$ overlayers on vicinal surfaces. . 127

Figure 5.6: Real space models of (a) the $M o(910)-c(2 \times 2) S$ surface

showing correlated sulfur ordering between neighboring

terraces, (b) the $\mathrm{Mo}(910)-\mathrm{c}(2 \times 2) \mathrm{S}$ surface showing

uncorrelated sulfur ordering between neighboring terraces

((b) appears to be the correct modei) and, (c) the

Mo(911) - $c(2 \times 2) S$ surface showing that the step direction

forces correlations between sulfur atoms on different terraces. . . 128

Figure 5.7 Diffraction patterns of $\mathrm{p}(2 \times 1) \mathrm{S}$ overlayer on (a) $\mathrm{Mo}(100)$,

(b) $M o(910)$ and (c) $M o(28,4,1)$ surfaces. . . . . . . . . . 130

Figure 5.8: Diffraction patterns of $\mathrm{Mo}(28,4,1)-(1 \times 1) \mathrm{S}$ surface. . . . . 132

Figure 5.9: Diffraction patterns of (a) clean $M O(910)$ and

(b) Mo(910) - (3 X 1)Co surfaces showing that only the domains

with the longer side of the unit cell (triple periodicity axis)

perpendicular to the step edges are formed.

Figure 5.10: Diffraction patterns of cobalt overlayers on the

$\mathrm{Mo}(100)-c(2 \times 2) S$ surface, (a) $\theta_{\mathrm{Co}_{0}}=0 \mathrm{ML}$, (b) $\theta_{\mathrm{Co}} \sim 1 \mathrm{ML}$

and (c) $\theta_{\mathrm{Co}} \sim 4 \mathrm{ML}$

Figure 5.11: Diffraction patterns of cobalt overlayers on the

$\mathrm{Mo}(100)-c(4 \times 2) S$ surface, (a) $\theta_{\mathrm{Co}_{0}}=0 \mathrm{ML}$ and

(b) $\theta_{\mathrm{Co}} \sim 1 \mathrm{ML}$.

Figure 5.12: Diffraction patterns of cobalt overlayers on the

$\mathrm{Mo}(100)-\mathrm{p}(2 \times 1) \mathrm{S}$ surface, (a) $\theta_{\mathrm{Co}_{0}}=0 \mathrm{ML}$ and

(b) $\theta_{\mathrm{Co}}-1 \mathrm{ML}$. 
Figure 6.1: Product accumulation curves for thiophene HDS over Mo(910) surfaces. $\mathrm{P}_{\mathrm{Th}} \sim 3$ Torr, $\mathrm{P}_{\mathrm{H}_{2}}-780$ Torr, $\mathrm{T}=613 \mathrm{~K}$.

Figure 6.2: Initial thiophene HDS rates over Mo(100), Mo(910), $\mathrm{Mo}(911)$ and $\mathrm{Mo}(28,4,1)$ surfaces.

$\mathrm{P}_{\mathrm{Th}} \sim 3$ Torr, $\mathrm{P}_{\mathrm{H}_{2}} \sim 780$ Torr, $\mathrm{T}=613 \mathrm{~K}$. . . . . . .

Figure 6.3: Temperature dependence of thiophene HDS over Mo(910) siufaces, $P_{T h}-3$ Torr, $P_{\mathrm{H}_{2}} \sim 750$ Torr.

Figure 6.4: Thiophene HDS rate as a function of the initial carbon coverage or $\operatorname{Mo}(28,4,1)$ surfaces. The rates are quoted per unit surface area of the initially clean $\operatorname{Mo}(28,4,1)$ suriace. $\mathrm{P}_{\mathrm{Th}} \sim 3 \mathrm{Torr}, \mathrm{P}_{\mathrm{H}_{2}} \sim 780$ Torr, $\mathrm{T}=613 \mathrm{~K}$.

Figure 6.5: Effect of cobalt on the thiophene HDS rate over $\mathrm{Mo}(100)$ surfaces. The observed rates are divided by the rate of the initially clean surface. They are not normalized to account for the fraction of the surface that is cobalt covered. The data point for the cobalt foil was taken from reference 8. $\mathrm{P}_{\mathrm{Th}} \sim 3$ Torr, $\mathrm{P}_{\mathrm{H}_{2}} \sim 780$ Torr, $\mathrm{T}=613 \mathrm{~K} . \quad . \quad .$.

Figure 6.6: Effect of cobalt on the thiophene HDS rate over Mo(910) surfaces. The observed rates are divided by the rate of the initially clean surface. They are not normalized to account for the fraction of the surface that is cobalt covereu. The data point for the cobalt foil was taken from reference 8.

$\mathrm{P}_{\mathrm{Th}} \sim 3$ Torr, $\mathrm{P}_{\mathrm{H}_{2}} \sim 780$ Torr, $\mathrm{T}=613 \mathrm{~K}$.

Figure 6.7: Effect of cobalt on the thiophene HDS rate over $\operatorname{Mo}(28,4,1)$ surfaces. The observed rates are divided by the rate of the initially clean surface. They are not nomalized to account 
for the fraction of the surface that i, cobalt covered. The data point for the cobalt foil was taken from reference 8.

$\mathrm{P}_{\mathrm{Th}} \sim 3$ Torr, $\mathrm{P}_{\mathrm{H}_{2}}-780$ Torr, $\mathrm{T}=613 \mathrm{~K} . \quad$. . . . . . 159 


\section{List of Tables}

Table 1.1: Sulfur levels found in crudes from various locations. . . . . . 8 Table 1.2: Thermodynamic data for thiophene hydrodesulfurization. . . . . 8

Table 1.3: Important features on the surface of hydrotreating catalysts. . . . 13

Table 3.1: Calculated entergies at which singlets and doublets are seen for monoatomic and diatomic steps on vicinal Mo(100) surfaces for the symmetrically equivalent $(01),(0 \overline{1}),(10)$ and $(\overline{1} 0)$ beams. . . . . . .

Table 3.2: Energies at which singlets and doublets are observed for the (01) beam for vicinal $\mathrm{Mo}(100)$ surfaces. . . . . . . . . . . 78

Table 3.3: Calculated number of terrace atoms on vicinal $\mathrm{Mo}(100)$ surfaces. $\quad .78$

Table 5.1: Experimental energies at which singlets and doublets are seen for the $(0 \overline{1})$ and equivalent beams for sulfur covered surfaces. The data given was obtained for the $\mathrm{Mo}(28,4,1)$ surface. The calculated energies listed for comparison were obtained by assuming that two atom height steps were present. . . . . 126

Table 5.2: $\quad$ Sulfur Overlayers on Molybdenum Single Crystal Surfaces. . . 133

Table 5.3: Ordered Structures formed by Cobalt Deposition on Clean and Sulfur Covered Molybdenum Surfaces. . . . . . . . . . 143 
The search for truth seemed easy at first, but ran into difficulty.

It is dark,

and a deep sea, with turbulent undercurrents

and surmounting waves, stands between us.

Those in a calm harbor,

how can they know what we must go through?

Hafez (Persian poet), 1350 


\section{Acknowledgements}

Even a way of a thousand miles starts with a step ${ }^{1}$.

Without the help of many people, the work reported in this thesis could never have been done. I would like to thank Professor Gabor A. Somorjai as well as all of the members of the Somorjai group for their very helpful discussions and explanations over the past four and a half years. Thank you Gabor for your guidance and allowing ine the freedom to learn and grow from my scientific mistakes. Other people who deserve special mention for their help and friendship include Matthias Wuttig, Ulrich Starke, Sabrina Fu; Mark Bussell, Michael Quinlan, Robert Hwang, Istvan Böszörményi, Jim Powers and Gerard Vurens.

I would especially like to acknowledge Mark Bussell for his patience in teaching me to operate the ultrahigh vacuum chamber used in this thesis. Pin Chen patiently showed me how to orient and polish single crystals. Matthias Wuttig critically reviewed most of my thesis. Thank you Matthias for your supportive friendship and your thoughtful suggestions. I am deeply grateful to Ulrich Starke who took the time to answer all of my questions about diffraction from stepped surfaces as well as shared my frustrations of drawing stepped surfaces using the SARCH program. Through the highs and lows of graduate school, I could count on Sabrina Fu to be there when I needed a friend. For that Sabrina, you have my deepest gratitude. Michael Quinlan always found the time to answer my day to day questions about surface science, catalysis and chemistry in general. He also reviewed most of chapters 4 and 6.

The support of all of the technical staff has been invaluable over the past few years. Gloria Osterloh and Brigid Tung were caring and helpful secretaries who made dealing with the UCB and LBL administrations, and life in general in the Somorjai group more pleasant. Bob Wright and Dan Colomb built many pieces of equipment and always were a 
constant and able source of help. In the electronic shop, Jim Severns and Bob Ybarra were especially helpful as were John Holthuis and John Jacobson in the materials processing shop.

On a more personal note, I acknowledge those dearest to me: my parents for having conficience in me, my relatives, my friends, my mentors and my homeland which provided the foundation of my education, the Republic of Trinidad and Tobago, West Indies.

I also thank AT\&T Bell Laboratories for financial support during my graduate work. This work was supported by the Director, Office of Energy Research, Office of Basic Energy Sciences, Materials Sciences Division, U.S. Department of Energy under contract No. DE-AC 03-76SF00098.

Berkeley, March, 1992

1. Chinese saying 


\section{Chapter One Introduction}

\subsection{General Concepts of Catalysis}

The classical definition of a catalyst is a substance which accelerates the rate of a reaction and can be recover'd chemically unchanged at the end of the reaction ${ }^{1}$. An alternative definition is a material in the presence of which a lower activation energy route is opened to a reaction. Catalysis is divided into homogeneous and heterogeneous catalysis. In homogeneous catalysis, the reactants and the catalyst are in the same phase. In heterogeneous catalysis, the catalyst is generally a solid surface and the reactants are in gaseous or liquid phase. Therefore the reaction is confined to the two dimensional surface of the catalyst. This thesis focuses on heterogeneous catalysis. Important concepts in catalysis are catalyst activity, structure sensitivity or insensitivity, selectivity and promotion or conversely poisoning. The activity of a catalyst is a measure of the extent that a catalyst accelerates the rate of a reaction. It is measured by the turnover frequency of the catalyst which is the number of product molecules produced per catalytic site per unit time. Ambiguities in determining the number of catalytic sites lead to ambiguities in defining and comparing turnover frequencies of different catalysts.

In a structure sensitive reaction it is not only the chemical identity of the catalyst that is important but also the structure of the catalytic surface. Previously the structure sensitivity of a reaction was probed by performing the reaction over catalysts ${ }^{2}$ with mean sizes of their particles generally ranging between 1 and $10 \mathrm{~nm}$. If the rate of the reaction 
changed with the mean catalyst particle size, the reaction was classified as being a structure sensitive reaction. In a structure insensitiv 2 reaction, the rate of the catalyzed reaction was independent of the particle size. Often the mean metal particle size was changed by sintering the catalysts, which can lead to unknown and uncharacterised changes in the surface structure ${ }^{3}$. On the different crystallographic faces of single crystals, the configuration of the exposed surface atoms are very different. This is demonstrated schematically in figure 1.1, where the top view of common low Miller index surfaces of a material with a body centered cubic unit cell (molybdenum for example) is shown. By performing catalytic reactions over such single crystal surfaces, we have a direct means of studying the effect of surface structure on catalytic activity and hence the structure sensitivity of a reaction ${ }^{2,4.5,6}$.

In many reactions involving complex molecules different types of bonds can be broken. In the hydrodesulfurization of thiophene for example, both carbon-carbon and carbon-sulfur bonds can be broken. Often it is desirable that specific types of bonds are broken so that the desired product is formed most abundantly. Examples of reactions involved ia carbon-carbon and carbon-sulfur bond cleavage are shown below.

$$
\begin{array}{lrl}
\text { hydrogenolysis } & \mathrm{RCH}_{2} \mathrm{CH}_{2} \mathrm{R}^{\prime}+\mathrm{H}_{2} \longrightarrow & \longrightarrow \\
\text { hydrodesulfurizaton } & \mathrm{R}-\mathrm{SH}+\mathrm{HCH}_{3} \longrightarrow \mathrm{R}^{\prime} \mathrm{CH}_{3}
\end{array}
$$

In the hydrogenolysis reaction shown, sulfur is not removed from the stream of organic molecules, but hydrogen is consumed. Since hydrogen is an expensive reactant, this reaction is very undesirable in industry. Therefore a catalyst with a high selectivity towards breaking a specific type of bond (in this case carbon-sulfur bonds) is chosen. The selectivity of a catalyst is a measure of the ability to catalyze the formation of desired products compared to undesired products. Additives can be introduced during the 

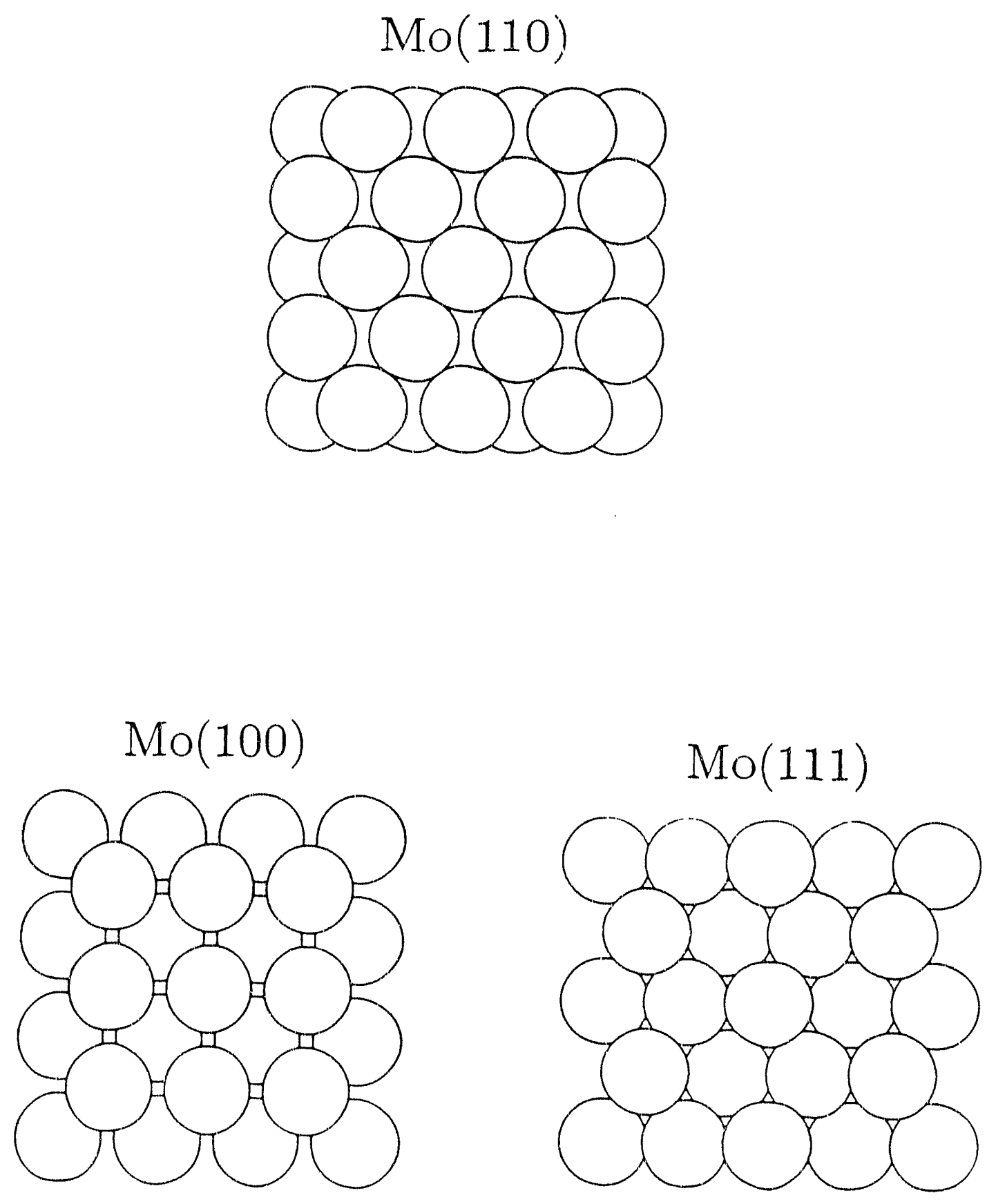

XBL $878-3472$

Figure 1.1: Top views of low Miller index surfaces of molybdenum. 
preparation of a catalyst. These acditives can markedly affect the activity, selectivity and lifetime of the catalyst. Additives that have a beneficial effect on the behavior of catalysts are called promoters while those that adversely affect the catalytic surface are termed poisons.

\subsection{A Surface Science Approach to Catalysis}

Heterogeneous catalysis is a surface phenomenon and therefore the tools of surface science are well suited to study model catalytic surfaces. Catalysis, at present is an empirical art that strongly relies on experience. If one wants to begin to understand catalysis on the atomic level, one has to understand the intermediates involved in reactions and the effects of surface structure and composition. This knowledge would enable the building of taylor made catalysts. A surface science approach to catalysis is based on the premise that knowledge of atomic composition and structure gained in UHV environments can be correlated with macroscopic parameters measured under catalytic conditions. Such an approach must allow UHV surface characterization and high pressure catalytic reactions to be performed in the same apparatus. An in situ cell capable of being sealed and pressurized with an atmosphere of gases was incorporated into the UHV. chamber used in this work. This combined UHV / catalytic system is shown in figures 2.3 and 2.4. The high pressure cell and reaction loop is described in section 2.2.2. Careful surface science experiments can allow the determination of the structure and composition of model surfaces, before, after and in principle even during chemical reactions.

In a thorough catalytic study, it is imperative to know the composition and chemical environment of elements in the surface region. Auger electron spectroscopy (AES) can be readily used to determine the surface elemental composition and $x$-ray 
photoelectron spectroscopy (XPS) ${ }^{7}$ is a ready probe of the chemical environment. $\hat{A}$ complete picture of the catalytic surface must also include a description of both the long and short range order of the surface as well as surface imperfections. The periodic arrangement of groups of atoms (unit cells) constitute the long range order of a surface. By observation of the low energy electron diffraction (LEED) $)^{7,8}$ pattern, the symmetry and size of the surface unit cell and thus the long range order can be determined. From analysis of the intensity of diffraction spots and comparison with theoretical intensities simulated for model structures, the bond angles and bond lengths in the surface region are determined. Thus the short range order of the surface can also be probed through diffraction studies.

As noted previously, in a structure sensitive reaction, the bonding arrangement in the surface has a pronounced effect on the extent to which a given structure sensitive reaction is catalyzed. Thus the geometric structure of the surface must be analyzed in order to evaluate the structure sensitive behavior of a reaction. Even when long range order is present on a surface, it is not perfect. Surfaces have imperfections which include substrate dislocations, steps of varying orientation and adsorbate islands of varying size, shape, height and distribution. While LEED readily allows the determination of the long range order, the above surface imperfections are more easily characterized with scanning tunneling microscopy (STM) ${ }^{9}$. Information about substrate dislocations is important since dislocations can serve as nucleation sites for growing islands. Dislocations can directly affect the rate of a catalyzed structure sensitive reaction. Steps also play a role in creating nucleation sites and affecting catalytic reactivity. Additionally, the orientation of the step can determine which ordered structures nucleate and grow on the terrace. The distribution of adsorbate islands provides information on adsorbate-adsorbate and adsorbate-substrate interactions. Also the distribution of islands dictates the availability of different sites for reacting gas molecules to bind to. Information on binding strength of adsorbates to 
substrates can be gained by a mass spectrometric technique called thermal desorption spectrometry.

Chemisorbed species are often present on catalytic surfaces. These can include preadsorbed atoms and molecules deposited on the surface to determine the effect of additives on the reactivity. The additives can either be promoters or poisons.

Alternatively, these chemisorbed species can include fragments formed on the surface during catalysis from decomposition and reaction. High resolution electron energy loss spectroscopy (HREELS) ${ }^{10}$ provides information on the chemical nature of adsorbates and their adsorption sites.

Of the techniques mentioned here, only scanning tunneling microscopy can be performed at atmospheric pressures. The others are used for characterization of the catalytic surfaces before and after reactions are performed. In this thesis low energy electron diffraction, Auger spectroscopy and thermal desorption spectrometry were used. These techniques are reviewed in the second chapter.

\subsection{Hydrodesulfurization}

Hydrodesulfurization ${ }^{11}$ is a catalyzed process in which sulfur is removed from organic molecules in petroleum through reaction with hydrogen. This reaction forms desulfurized organic molecules and liberates hydrogen sulfide.

$$
\begin{aligned}
& \text { sulfur compounds }+\mathrm{H}_{2} \longrightarrow \text { desulfurized products }+\mathrm{H}_{2} \mathrm{~S} \\
& \text { in petroleum }
\end{aligned}
$$

The hydrogen sulfide liberated through this process must be removed safely. It would be 
hazardous to our environment if the hydrogen sulfide was allowed to escape. Industrially it is removed using the Claus process. This process involves the air oxidation of a fraction of the hydrogen sulfide to sulfur dioxide. This sulfur dioxide is then reacted with the remaining hydrogen sulfide yielding elemental sulfur and water.

Hydrodesulfurization is just one of four processes included under the heading of hydrotreating. Hydrotreating is used to remove harmful contaminants from crude oil and includes in addition to hydrodesulfurization $(\mathrm{HDS})$, hydrodenitrogenation $(\mathrm{HDN})^{12}$, hydrodeoxygenation $(\mathrm{HDO})^{13}$ and hydrodemetallation $(\mathrm{HDM})^{14}$. About one third of the total crude oil extracted is hydrotreated to remove sulfur, nitrogen, oxygen and metal contaminants ${ }^{15}$. United States sales for hydrotreating catalysts exceeded seventy million dollars in 1988 and are expected to continue increasing. Japanese sales for hydrodesulfurization catalysts alone exceeded seventy million dollars in $1987^{16}$. Hydrodesulfurization of light petroleum feedstocks is necessary because sulfur poisons the platinum containing catalysts used in reforming. While in the case of light petroleum fractions that give rise to gasolines, the main incentive for hydrodesulfurization is economic (protecting the platinum catalysts), in the case of heavy petroleum fractions, the main incentive is environmental ${ }^{17}$. Diesel and jet fuels are derived from heavy petroleum fractions. The combustion of sulfur containing fuels is one of the sources of sulfur dioxide pollution in the atmosphere. This problem is most severe in western Europe, the eastern United States and Japan. Sulfur dioxide has been implicated as a precursor to a $a \cdot$ id rain formation. Acid rain is known to have devastating effects on lakes, forests, other natural ecosystems and buildings. Reduction of the sulfur content of diesel and jet fuels must be achieved to reduce acid rain formation. A major source of sulfur dioxide emissions is the burning of coal in power plants.

The sulfur content of various crudes are shown in table $1.1^{18}$. The major sulfur contanning contaminants in crude oil in order of decreasing abundance are thiols (R-SH), 
Table 1.1: Sulfur levels found in crudes from various locations.

\begin{tabular}{|c|c|}
\hline Location & $\begin{array}{c}\text { Sulfur content } \\
\text { \% by weight }\end{array}$ \\
\hline Trinidad and Tobago, W.I. & 0.40 \\
Boscan, Venezuela & 5.53 \\
Oficina, Venezuela & 0.59 \\
Poza Rico. Mexico & 1.77 \\
Mantantiales, Chile & $<0.10$ \\
Swanson River, Alaska, U.S.A. & 0.16 \\
Long Beach, California, U.S.A. & 1.29 \\
\end{tabular}

Table 1.2: Thermodynamic data for thiophene hydrodesulfurization.

\begin{tabular}{|l|c|c|c|}
\hline Reaction & $\begin{array}{c}\Delta \mathrm{H}^{\circ} \text { 298 } \\
(\mathrm{kcal} / \mathrm{mol})\end{array}$ & $\begin{array}{c}\Delta \mathrm{S}_{298} \\
(\mathrm{cal} / \mathrm{mol})\end{array}$ & $\begin{array}{c}\Delta \mathrm{G}^{\cdot}{ }^{298} \\
(\mathrm{kcal} / \mathrm{mol})\end{array}$ \\
\hline $\mathrm{C}_{4} \mathrm{H}_{4} \mathrm{~S}+\mathrm{n} \mathrm{H}_{2} \longrightarrow$ butadiene $+\mathrm{H}_{2} \mathrm{~S}$ & -5.9 & -13.3 & -1.9 \\
$\mathrm{C}_{4} \mathrm{H}_{4} \mathrm{~S}+\mathrm{n} \mathrm{H}_{2} \longrightarrow$ 1-butene $+\mathrm{H}_{2} \mathrm{~S}$ & -32.2 & -37.6 & -21.0 \\
$\mathrm{C}_{4} \mathrm{H}_{4} \mathrm{~S}+\mathrm{nH}_{2} \longrightarrow \mathrm{t}-2$-butene $+\mathrm{H}_{2} \mathrm{~S}$ & -35.0 & -40.2 & -22.9 \\
$\mathrm{C}_{4} \mathrm{H}_{4} \mathrm{~S}+\mathrm{nH}_{2} \longrightarrow$ c-2-butene $+\mathrm{H}_{2} \mathrm{~S}$ & -34.0 & -39.2 & -22.2 \\
$\mathrm{C}_{4} \mathrm{H}_{4} \mathrm{~S}+\mathrm{nH}_{2} \longrightarrow$ butane $+\mathrm{H}_{2} \mathrm{~S}$ & -62.4 & -68.2 & -41.9 \\
\hline
\end{tabular}




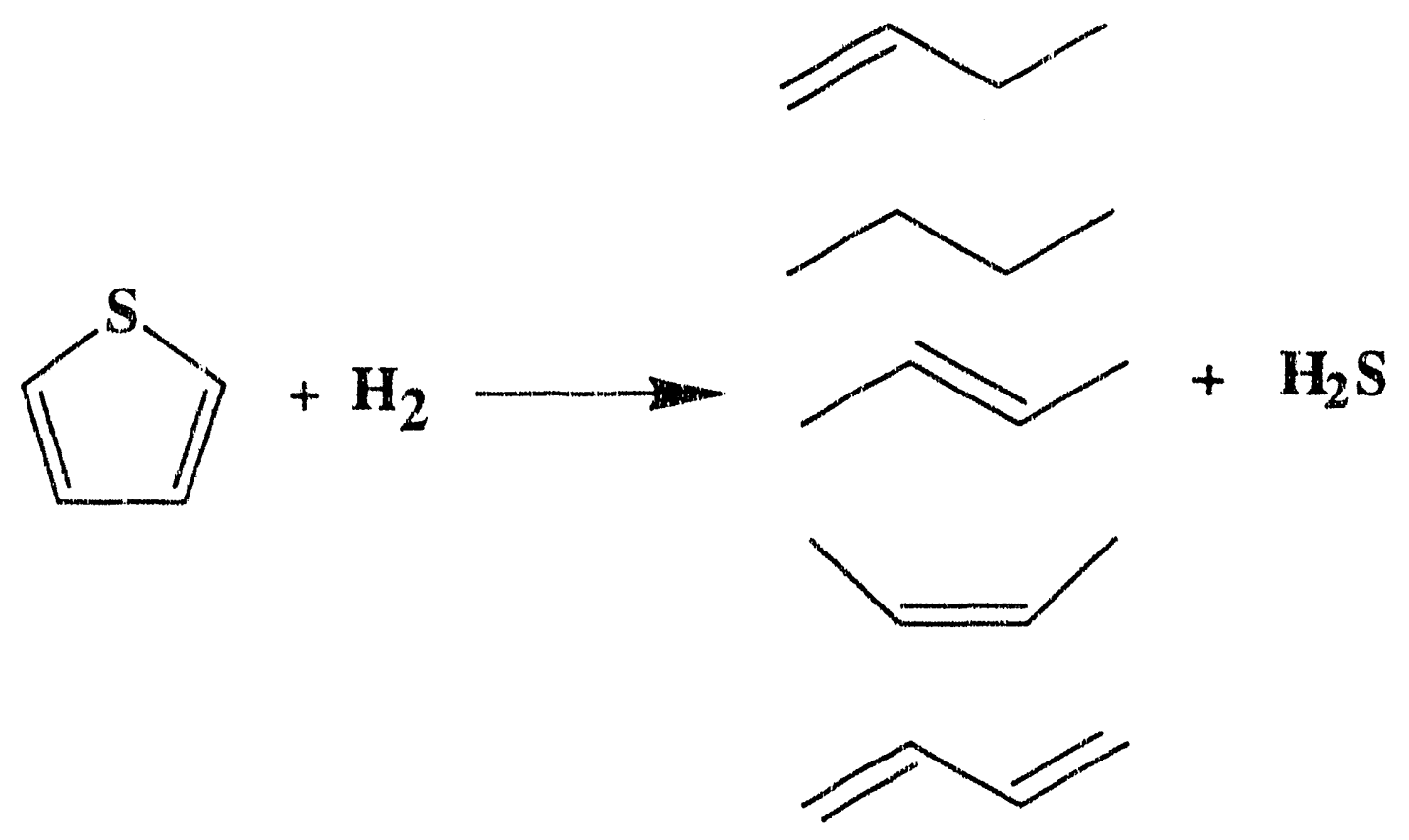

Figure 1.2: Reaction for thiophene hydrodesulfurization over molybdenum single crystal surfaces. 
sulfides (R-S-R'), disulfides (R-S-S-R') and thiophenes. Of the contaminants above, the thiophene derivatives are the most difficult to desulfurize. In this thesis, thiophene itself was chosen as the test molecule. Thiophene is a convenient test molecule since it has a sufficiently high vapor pressure $(\sim 80$ Tort) that it can be introduced into the reaction loop and kept in vapor form without heating the entire loop. The reactions involved in thiophene hydrodesulfurization and the thermodynamic data for these reactions are shown in figure 1.2 and table 1.2 respectively.

Industrial hydrodesulfurization catalysts can be prepared by pore volume impregnation of $\gamma-\mathrm{Al}_{2} \mathrm{O}_{3}$ with aqueous solutions of ammonium heptamolybdate $\left(\left(\mathrm{NH}_{4}\right)_{6} \mathrm{MO}_{7} \mathrm{O}_{24}\right)$ and cobalt nitrate $\left(\mathrm{Co}\left(\mathrm{NO}_{3}\right)_{2}\right)$. Through drying and calcination steps, the ammonium heptamolybdate is converted to molybdenum trioxide $\left(\mathrm{MoO}_{3}\right)$ and cobalt is converted into an oxidic forn $(\mathrm{CoO})$. A typical industrial composition is $4 \% \mathrm{CoO}$ and $12 \% \mathrm{MoO}_{3}$ on the support. This oxidic precursor is sulfided to form the activated catalyst by using for example $\mathrm{H}_{2} \mathrm{~S} / \mathrm{H}_{2}$ or a liquid feed of sulfur containing molecules and hydrogen. On these sulfided catalysts, it is generally accepted that molybdenum is present as molybdenum sulfide crystallites ${ }^{19}$. As shown schematically in figure 1.3 , these crystallites can lie with their basal plane either parallel or perpendicular to the support ${ }^{20,21}$. It is suggested that these crystallites are attached to the support through oxysulfided molybdenum species. The situation is more complex in the case of cobalt as schematically depicted in figure.1.4. Cobalt aluminate species are found. These are formed by the trapping of cobalt ions in the alumina support ${ }^{22}$. Different cobalt sulfides are also seen on a working catalyst. $\mathrm{Cog}_{9} \mathrm{~S}_{8}, \operatorname{CoS}_{1+\mathrm{x}}$, a species proposed to be similar to the $\beta-\operatorname{CoS}_{1.097}$ phase, a sulfide species where cobalt has a distorted tetrahedrical coordination ${ }^{19}$ and a Co-Mo- $\mathrm{S}^{23}$ species in which cobalt decorates the $\mathrm{MOS}_{2}$ edges have all been reported. The known temary phase $\left(\mathrm{CoMo}_{2} \mathrm{~S}_{4}\right)$ is usually not present. The species found on the surface of these catalysts are summarized in table $1.3^{15}$. While molybdenum is a necessary 


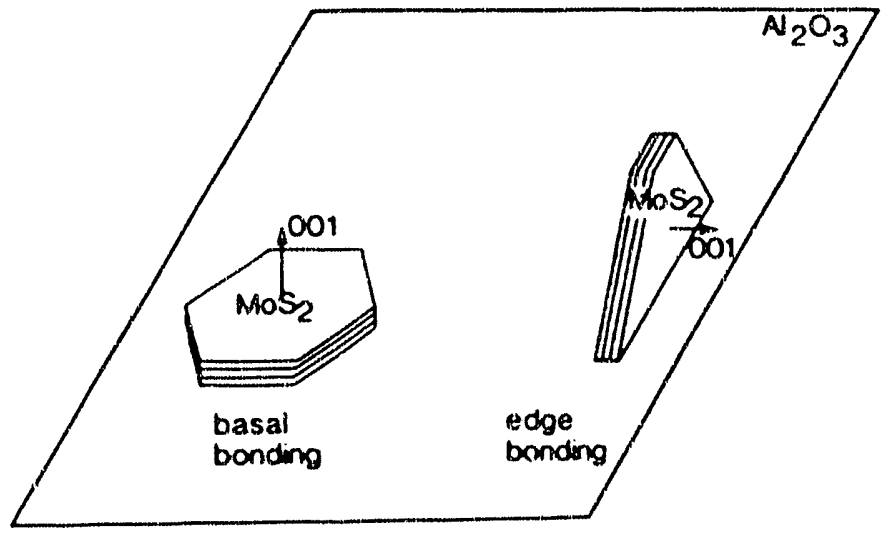

(a)
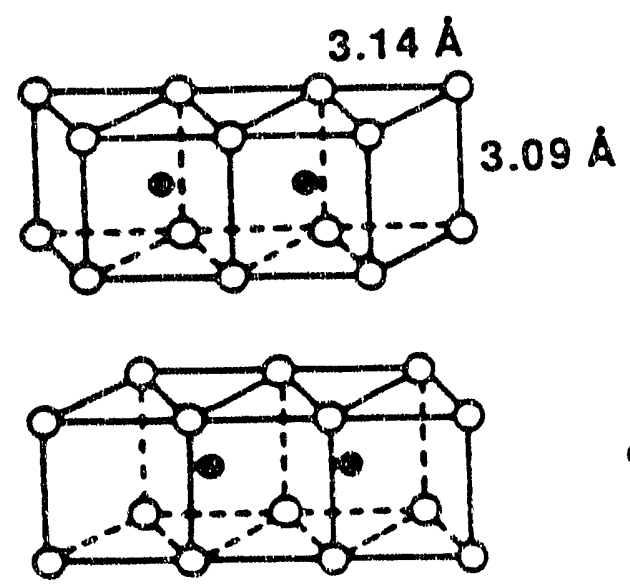

OS

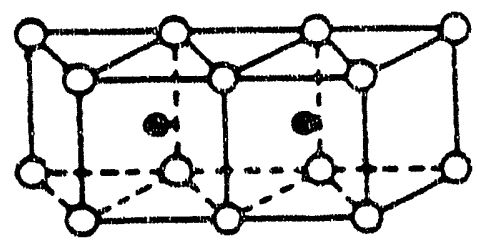

(b)

Figure 1.3: Schematics of a) edge and basal bonding and b) structure of $\mathrm{MoS}_{2}$. 


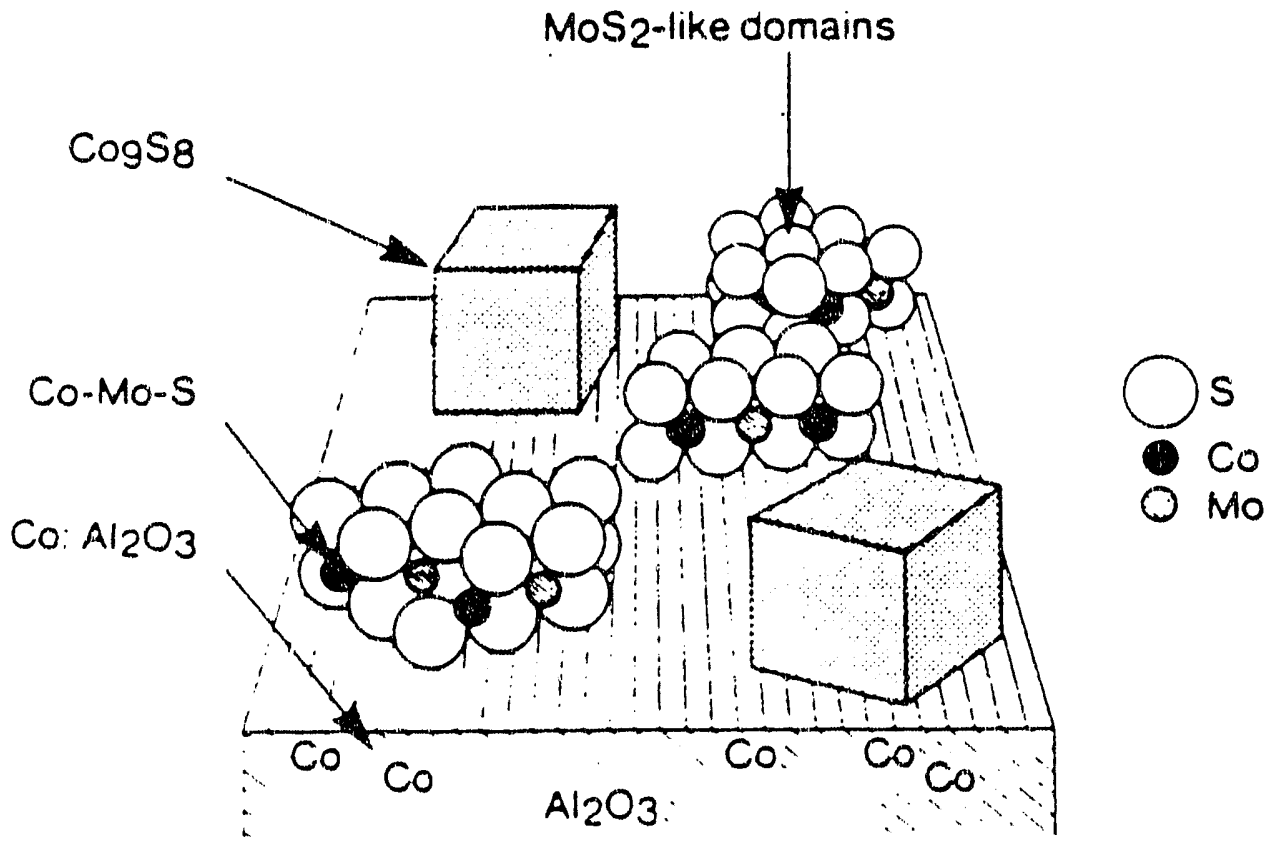

Figure 1.4: The different cobalt phases coexisting on supported HDS catalysts. 
Table 1.3: Important features on the surface of hydrotreating catalysts.

\section{Molybdenum species}

$\mathrm{MoS}_{2}$ poorly or well crystallized

Coordination of superficial Mo atoms

Oxysulfided Mo species

[ $\mathrm{MoO}_{3}$ : normally absent]

$\left[\mathrm{Al}_{2}\left(\mathrm{MoO}_{4}\right)_{3}:\right.$ normally absent]

Cobalt species

$\mathrm{Cog}_{8} \mathrm{~S}_{8}$

$\operatorname{CoS}_{1+\mathrm{x}}\left(\beta-\operatorname{CoS}_{1.097}\right.$ or similar $)$

$\operatorname{CoS}_{\mathrm{x}}$ with tetrahedral distorted coordination

$\mathrm{Co}$ in $\mathrm{Al}_{2} \mathrm{O}_{3}$

Mixed Co-Mo-S species

"Co-Mo-S" phase

[CoMoO $4:$ normally absent]

[CoMon $\mathrm{S}_{4}$ : normally absent]

Coordination of Co on surface?

Other surface functions

SH

Acidity; $\mathrm{OH}$

Deposits

Carbonaceous deposits

Polysulfide species

Metal deposits (sulfides) 
constituent of hydrodesulfurization catalysts, cobalt is not. Cobalt alone does not have a significant activity. Since combinations of cobalt and molybdenum have higher activities than molybdenum alone, cobalt is called a promoter. Alternately, tungsten can be used in the place of molybdenum and nickel in the place of cobalt. Hydrodesulfurization reactions are thermodynamically favored (with exothermic heats of formation between 10 and 20 $\mathrm{kcal}$ per mole of hydrogen consumed) under the conditions used in industry. Industrial operating temperatures and pressures are typically between 573 and $723 \mathrm{~K}$ and 20 to 200 atmospheres respectively. The more severe processing conditions are used for the heavy petroleum fractions.

Catalyst poisoning generally results either from coke deposition or plugging of the pores of the catalysts with solid metal sulfides. Vanadium and nickel sulfides are the major culprits. Heavy petroleum fractions can sometimes contain significant amounts of these metal contaminants and as a result deactivation of these catalysts can occur after less than one year of use. For comparison, catalysts used for light petroleum fractions can have lifetimes of about ten years. Even though coke deposition both plugs the pores of the catalyst and poisons the catalytic surface, the reaction temperature can be increased to maintain a reasonable activity. Periodically, coke is burnt off the catalyst to regenerate it. The deposition of metal sulfides is irreversible. The technology involved in the hydrodesulfurization of light petroleum fractions is well developed in industry. The cost of the catalyst accounts for less than $10 \%$ of the processing costs. There is therefore little economic incentive to improve these catalysts. This is not the case for the technology involved in sulfur removal from heavy petroleum fractions. As political and ervironmental forces and the neut to use heavier sources such as tar sands, oil shales, coal and biomass increase, improved catalysts to meet these new demands will have to be developed. 


\subsection{Promoter Role of Cobalt}

Although there have been numerous studies aimed at elucidating the promoter role of cobalt, this question is still wide open. Understanding the role of cobalt is the major motivation for the work done in this thesis. A major problem is the determination of the number of active sites on the catalyst surface. If the number of active sites could be determined, some of the proposed models could be eliminated. In the early 1980's, some workers reported a correlation between the oxygen adsorption capacity of the unpromoted molybdenum sulfide $\left(\mathrm{MoS}_{2}\right)$ catalysts and the hydrodesulfurization reactivity ${ }^{24}$. However a later study indicated that oxygen adsorption on these catalysts is only stable at low temperatures. At temperatures above $195 \mathrm{~K}$, the oxygen capacity increases, but this increase has been shown to be due to the slow oxidation of molybdenum sulfide. In especially the cobalt promoted catalysts there was bulk oxidation of cobalt phases. The degree of oxidation depended on the chemisorption temperature ${ }^{25}$. Other studies have also discredited the use of oxygen in determining the number of catalytically active sites ${ }^{26}$. Preliminary studies have suggested that preferential adsorption of $\mathrm{CO}$ on $\mathrm{MoS}_{2}$ (rather than the alumina support) at $273 \mathrm{~K}$ might be used to determine the active surface area ${ }^{27}$, but this approach awaits further careful confirmation. $\mathrm{CO}$ has the advantage that it does not alter the catalyst.

Some of the suggestions to explain the promoter role of cobalt are described briefly. Topsøe and coworkers showed by infrared and volumetric studies of NO adsorption that on cobalt promoted molybdenum sulfide catalysts, cobalt is located on the molybdenum ions on the edges of the molybdenum sulfide crystallites ${ }^{23}$. They showed quite convincingly that when the cobalt coverage was increased, the infrared intensity due to adsorption of NO on cobalt increased, while the intensity due to adsorption of NO on molybdenum decreased. It must be noted that NO oxidizes these sulfides, and as a result 
changes the catalysts, possibly complicating the chemisorption data. A later extended $x$-ray absorption fine structure (EXAFS) study further clarified that although the cobalt edge atoms were located in the same plane as the molybdenum atoms, they were not on perfect edge molybdenum sites, but somewhat displaced ${ }^{28}$. The higher turnover frequencies measured for promoted catalysts were attributed to the presence of cobalt on these sites in so called Co-Mo-S structures. Chianelli and coworkers have suggested that there is an electronic interaction between cobalt and molybdenum that beneficially influences the catalytic activity by increasing the electron density in the highest occupied molecular orbital of molybdenum ${ }^{29}$.

Prins and coworkers ${ }^{30}$ have suggested that for carbon.(instead of $\gamma-\mathrm{Al}_{2} \mathrm{O}_{3}$ ) supported catalysts, there is an increased dispersion of cobalt sulfide $\left(\mathrm{Co}_{9} \mathrm{~S}_{8}\right)$ (proposed to be the phase responsible for the higher turnover frequency) over molybdenum sulfide (proposed in this model to act as a support). In these carbon supported systems, cobalt has the same Mössbauer signature as it does in the Co-Mo-S phase, suggesting that it is in a similar environment ${ }^{31,32}$. Other workers have shown that catalysts containing mostly cobalt sulfide have much lower turnover frequencies than cobalt promoted molybdenum catalysts. These observations have been used to argue against cobalt sulfide being the active phase. However in these catalysts the dispersion of cobalt sulfide is not reported to be exceptionally high as it is in the case of Prins and coworkers. This discrepancy points once again to the need to determine the number of catalytically active sites in hydrodesulfurization catalysts.

Other explanations have been proposed including the role of cobalt as a textural promoter $^{33}$. As a textural promoter, it has been proposed that cobalt ions induce a surface reconstruction of the edges of the molybdenum sulfide layers. This reconstruction supposedly increases the number of molybdenum cations exposed. These cations are the accepted catalytic sites for the unpromoted catalyst and therefore an increase in their 
numbers would lead to an enhanced activity. But in industriy, a ten to thirty fold enhancement can be seen for the promoted catalysts over their non-promoted counterparts. It is highly questionable as whether any such reconstruction could lead to a ten to thirty fold increase in the number of exposed molybdenum cations.

There have been no previous studies aimed at understanding the effect of cobalt on the more tractable model single crystal catalysts. In this thesis, the surface interactions between cobalt and / or sulfur or carbon overlayers on molybdenum single crystal surfaces are investigated by depositing known amounts of these adsorbates on the molybdenum surface from the vapor phase. Thiophene hydrodesulfurization reactions are performed over these different surfaces to correlate the surface structure and composition with the measured catalytic activity.

\subsection{Molybdenum Single Crystals as Model HDS Catalysts: Strengths and Limitations}

In this thesis, sulfur, carbon and cobalt overlayers were deposited on molybdenum single crystal surfaces. These surfaces were used as model catalysts in the study of the effect of cobalt on thiophene hydrodesulfurization. The validity of molybdenum single crystals as model catalytic surfaces must be addressed. Comparisons between the use of surface science and single crystals, and the techniques used to study "real" catalysts must also be addressed. We must not lose sight of the inadequacies of our model catalytic surfaces. In the industrial catalyst neither molybdenum nor cobalt have oxidation numbers of zero as they do in our model system. Chemisorbed sulfur on molybdenum should not be considered to be molybdenum sulfide, and one cannot assume that the interaction of cobalt with molybdenum metal will be similar to the interaction of cobalt with 
molybdenum sulfide. In studies of ammonia synthesis, where iron is the actual catalyst used in industry, the use of metal single crystals is easier to justify.

Another problem encountered when applying surface science to catalytic studies is the inherent pressure gap between the two fields. Surface preparation and characterization is done at pressures of $10^{-9}$ Torr or less, while catalytic tests are run at pressures of one atmosphere or more. Nevertheless, the use of clean and modified single crystals can provide interesting and potentially useful information ${ }^{3,34}$. The structure and composition of these model surfaces can be readily characterized. The relationship between the catalytic activity and the initial surface composition and structure can be determined. Hydrodesulfurization catalysts are very complex and indeed if we are to understand them we must use simplified systems. Molybdenum sulfide has a layered structure with weak Van der Waals forces between the basal sulfur planes (figure 1.3). Cleavage generally occurs between the basal planes. Molybdenum sulfide itself was not used in this thesis because previous work in our group suggested that the single crystals which were obtainable exposed only the basal plane which was found to be inactive ${ }^{35}$. This led to the use of molybdenum metal single crystals on which sulfur was deposited ${ }^{36,37}$.

There are techniques available for the study of "real" catalysts under reaction conditions. However these techniques are not without their problems. Infrared and Mössbauer emission spectroscopy have been successfully used to study "real" hydrodesulfurization catalysts. By pressing samples into disks, actual high surface area catalysts can be studied with infrared spectroscopy. Surface groups and adsorbed molecules on the surface of the catalyst can be studied under industrial reaction conditions (high pressures and temperatures) providing an appropriate cell is available ${ }^{38}$. The adsorption of probe molecules such as $\mathrm{CO}$ and NO can be studied. Such probe molecules can provide valuable information about the nature and environment of atoms and ions exposed on the catalytic surface. Indeed, it was infrared studies of NO adsorption on 
cobalt promoted molybdenum sulfide catalysts that Topsøe et al..$^{23}$ used to show the location of the promoter ions on the edges of the molybdenum sulfide crystallites. However, oxide supports such as $\gamma-\mathrm{Al}_{2} \mathrm{O}_{3}$ strongly adsorh infrared frequencies, making it impossible to take spectra below $1000-1100 \mathrm{~cm}^{-1}$ for oxide supported systems. Because of the inherent complexity of these systems, infrared studies do not always yield conclusive and unambiguous information about adsorbates and the nature of the catalytic surface. Mössbauer spectroscopy has also been extensively used in the study of hydrodesulfurization catalysts. Mössbauer has the capability of showing the coexistence of different cobalt phases but is limited in that it only has application with a handful of elements ${ }^{39,40}$. In situ Mössbauer studies first identified the Co-Mo-S surface phase that the Topsøe group propose to be responsible for the higher activity of promoted catalysts. Mössbauer however, cannot provide detailed structural information about this non-bulklike Co-Mo-S phase.

\subsection{Organization of Thesis}

The work performed in this thesis has atternpted, using the tools of surface science, to correlate the thiophene hydrodesulfurization activity over clean and adsorbate covered molybdenum single crystal surfaces with the initial structure and composition of these surfaces. In order to do so, the effect of surface steps and of adsorbates such as cobalt, sulfur and carbon have been investigated. This thesis is organized in the following manner. This chapter has described the motivation of this work as well as general considerations in catalysis, surface science and the intermarriage of these two fields. The combination of surface science and catalysis dictates the experimental set up that can be used. These considerations, the apparatus and the techniques used are discussed in the 
following chapter. The next three chapters provide a systematic study of the structure of the clean surfaces (chapter 3), adsorbate covered surfaces (chapter 5), and the growth of adsorbates (chapter 4) on these surfaces. In chapter 4, carbon monoxide is used to probe the growth of cobalt on clean and sulfur covered molybdenum surfaces. Finally in chapter 6, thiophene hydrodesulfurization over the clean and adsorbate covered, flat and vicinal molybdenum surfaces characterized in earlier chapters are discussed.

\section{References}

[1] I. N. Levine, Physical Chemistry, 2nd ed. McGraw-Hill, New York, (1983).

[2] M. Boudart and G. Djéga-Mariadassou, Kinetics of Heterogeneous Catalytic Reactions, Princeton University Press, Princeton, NJ, (1984).

[3] G. A. Somorjai, Chemistry in Two Dimensions: Surfaces, Cornell University Press, Ithaca NY, (1981).

[4] M. E. Bussell, A. J. Gellman and G. A. Somorjai, J. Catal. 110423 (1988).

[5] M. Assher and G. A. Somorjai, Surf. Sci. 143 L389 (1984).

[6] N. Spencer, R. C. Schoonmaker and G. A. Somorjai, Nature 294643 (1981).

[7] G. Ertl and J. Küppers, Low Energy Electrons and Surface Chemistry, 2nd ed. VCH, Germany, (1985).

[8] M. A. VanHove, W. H. Weinberg and C. -M. Chang, Low-Energy Electron Diffraction, Springer-Verlag, Berlin Heidelberg, (1986).

[9] G. Binnig, H. Rohrer, Ch. Gerber and E. Weibel, Phys. Rev. Lett. 4957 (1982).

[10] H. Ibach and D. L. Mills, Electron Energy Loss Spectroscopy and Surface Vibrations, Academic Press, New York, (1982).

[11] R. Prins, V. H. J. de Beer and G. A. Somorjai, Catal. Rev. Sci. Eng. 311 (1989). 
[12] H. Schulz, M. Schon and W. M. Rahman, Stud. Surf. Sci. Catal. 27201 (1986).

[13] C. L. Lee and D. F. Ollis, J. Catal. 87 325, 332 (1984).

[14] R. A. Ware and J. Wei, J. Catal. 93 100, 122, 135 (1985).

[15] B. Delmon, Proceedings of the Conference on Catalysis in Petroleum Refining, Kuwait, March 5-8, 1989.

[16] Chemical Week, p.20 June 29, 1988, McGraw-Hill.

[17] B. C. Gates, J. R. Katzer, G. C. Schuit, Chemistry of Catalytic Processes, Chap. 5 McGraw-Hill, (1979).

[18] C. M. McKinney, Petroleum Processing Handbook, Chap. 2, McGraw-Hill, (1967).

[19] B. Delmon, Chemistry and Uses of Molybdenum, Proc. Climax 3rd. Int. Conf., Climax Mo Cy. p. 73, Ann Arbor Michigan (1979)

[20] F. Delannay, Appl. Catal. 16135 (1985).

[21] T. F. Hayden and J. A. Dumesic, J. Catal. 103366 (1987).

[22] R. R. Chianelli, Cat. Rev.-Sci. Eng. 26361 (1984).

[23] N.-Y Topsøe and H. Topsøe, J. Catal. 84386 (1983).

[24] S. J. Tauster, T. A. Pecoraro and R. R. Chianelli, J. Catal. 63515 (1980).

[25] W. Zmierczak, G. MuraliDhar and F. E. Massoth, J. Catal. 77432 (1982).

[26] J. Bachelier, J. C. Duchet and D. Cornet, Bull. Soc. Chim. Belg. 901301 (1981).

[27] J. Bachelier, M. J. Tilliette, M. Cornac, J. C. Duchet, J. C. Lavalley and D. Cornet, Bull. Soc. Chim. Belg. 93743 (1984).

[28] H. Topsøe, B. S. Clausen, N.-Y. Topsøe, E. Pederson, W. Niemann, A Müller, H. Bögge, B. Lengeler, J. Chem. Soc., Faraday Trans. 1, 832157 (1987).

[29] S. Chianelli and R. R. Harris, J. Catal. 9817 (1986).

[30] V. H. J. de Beer, J. C. Duchet and R. Prins, J. Catal. 72369 (1981).

[31] S. M. A. M. Bowens, D. C. Koningsberger, V. H. J. de Beer and R. Prins, 
Bull. Soc. Chim. Belg, 96951 (1987).

[32] A. M. van der Kraan, M. W. J. Crajé, E. Gerkema, W. L. T. M. Ramselaar and V. H. J. de Beer, Appl. Catal. L 7 - L 10 (1988),

[33] A. J. A. Konnings, A. Valster, V. H. J. de Beer and R. Prins, J. Catal. 76466 (1982).

[34] M. Boudart, Chemtech, 688 November 1986,.

[35] M. Salmeron, G. A. Somorjai, A. Wold, R. R. Chianelli and K. S. Liang, Chem. Phys. Lett. 90105 (1982).

[36] A. J. Gellman, Ph.D. thesis, University of California, Berkeley (1985).

[37] M. E. Bussell, Ph.D. thesis, University of California, Berkeley (1988).

[38] J. B. Peri, Catalysis- Science and Technology Chap.3, Springer-Verlag Berlin Heidelberg NewYork Tokyo (1984).

[39] C. Wivel, R. Candia, B. Clausen, S. Mørup and H. Topsøe, J. Catal. 68453 (1981).

[40] H. Topsøe, B. Clausen, R. Candia, C. Wivel and S. Mørup, J. Catal 68433 (1981). 


\section{Chapter Two Experimental}

\subsection{Introduction}

In the last chapter, we outlined the basic goal of this thesis which is to obtain a better undustanding of an important catalyst by combining surface analytical techniques with high pressure studies. These studies allow the determination of reaction rates over different surfaces which are previously charaçerized to determine their structure, growth and composition. This combination puts severe constraints on the apparatus that can be used. In the following paragraphs, we will describe some of the considerations which influence the design of the experimental system. The apparatus and its main features are described in section 2.2. In section 2.3, a description of the applied surface analytical techniques is provided while section 2.4 outlines the sample preparation. The procedures involved in high pressure reactions are described in section 2.5 .

Most surface analytical techniques, including low energy electron diffraction (LEED) and Auger electron spectros opy (AES) require the detection of low energy electrons. The intere ction of low energy electrons with solids is shown iru figure $2.1^{1}$. In this figure, the spctrum of backscattered electrons $(N(E))$ from a solid after bombardrnent with $2 \mathrm{KeV}$ electrons is depicted. The spectrum is divided into several characteristic. regions. There is an elastic peak produced by primary electrons that are elastically backscattered. Withirn less than $1 \mathrm{eV}$ of the elastic electrons, are the electrons that have lost energy by excitation of surface vibrations (phonons). Electrons which have lost energy to 


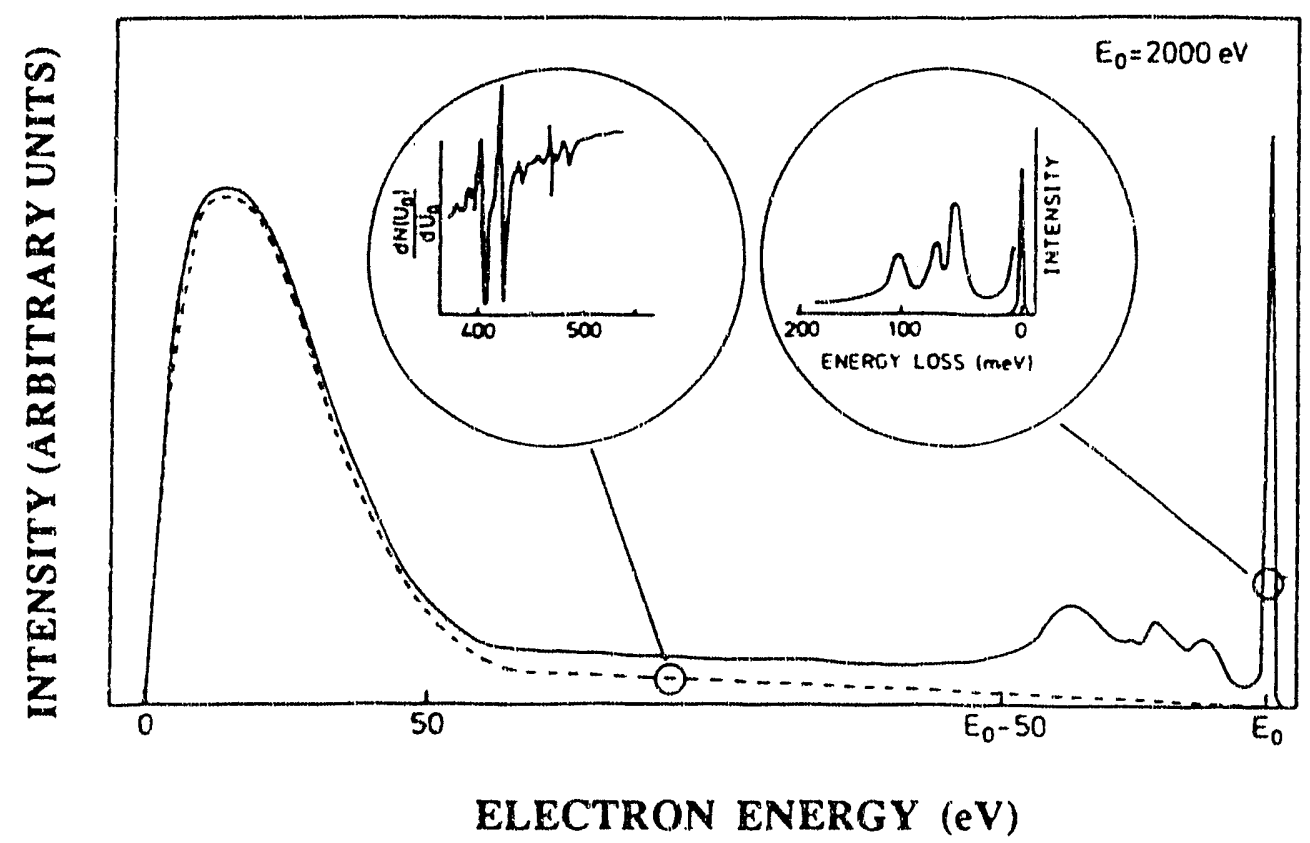

Figure 2.1: Spectrum of backscattered electrons from a solid after bombardment with $2 \mathrm{KeV}$ electrons.

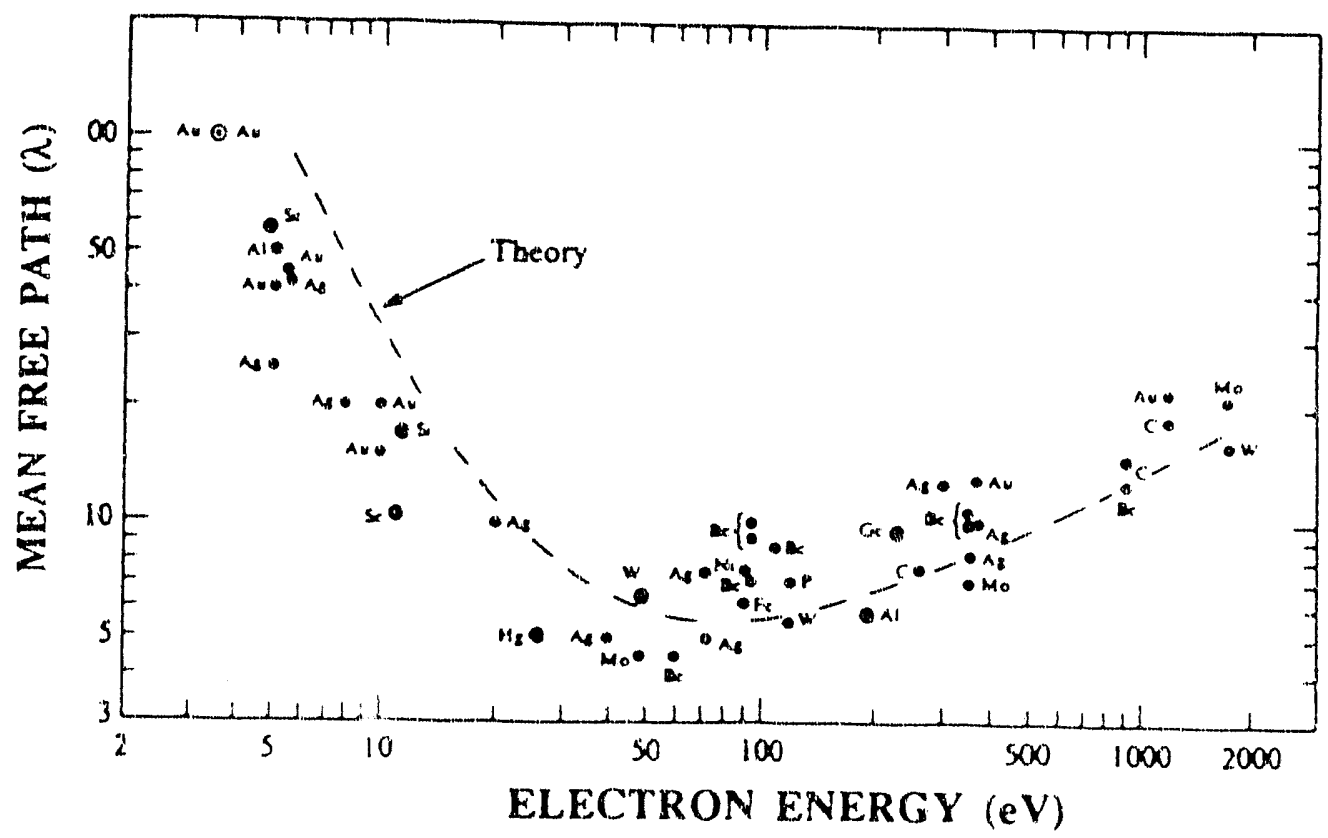

Figure 2.2: Universal curve of electron mean free path in solids. 
electronic excitation appear within $50 \mathrm{eV}$ of the primary energy. Superimposed on a relatively smooth background in the medium energy range, are small peaks caused by emission of Auger electrons. Most of the backscattered electrons are secondary electrons (shown by the dashed line). These electrons dominate the low energy $(<50 \mathrm{eV})$ end of the spectrum. This tern "secondary electrons" describes electrons that were bound in the solid and which have gained a small but sufficient amount of energy to escape after inelastic collisions with the primary electrons.

The universal curve of electron mean free path in figure $2.2^{2}$ shows that electrons with kinetic energies between 15 and $1000 \mathrm{eV}$ have inelastic mean free paths of less than $10 \AA$ in most solids. Clearly, electrons leaving the solid with these kinetic energies have only originated from the top few surface layers. Therefore these electrons provide information exclusively about the surface region and not about the more abundant bulk region. This is an important consideration since the number of surface atoms is of the order of $10^{15}$ atoms $/ \mathrm{cm}^{2}$ compared to $10^{23}$ atoms $/ \mathrm{cm}^{3}$ for the bulk.

The detection of low energy electrons necessitates the use of ultra high vacuum (UHV) conditions. Electrons with these kinetic energies have shor $(\sim 2000 \AA)$ mean free paths in air. Under ulerahigh vacuum pressures $\left(<10^{-9} \mathrm{Torr}\right)$, the mean free path of these electrons is of the order of $10^{5}$ meters. Therefore these electrons can leave the solid and be collected at a detector several centimeters away without colliding with ambient gas molecules.

Ultra high vacuum also allows the preparation of atomically clean surfaces. At pressures around $10^{-6} \mathrm{Torr}$, the kinetic theory of gases indicates that the collision rate of gas molecules (for example nitrogen) is sufficiently high to cover the surface with a monolayer of molecules in one second if every molecule that collides with the surface can stick to it. At pressures of $10^{-9}$ Torr and lower, surfaces can be kept atomically clean for several minutes and possibly hours depending on the sticking coefficient of the gas under 
consideration.

\subsection{Apparatus}

Experiments reported in this thesis were performed using the ultra high vacuum system shown in figures 2.3 and 2.4. The major components of the system are described below.

\subsubsection{Sample Manipulator}

The manipulator consisted of a high precision xyz translator (part \# VZHPT205A) purchased from Vacuum Generators in 1990. This module has the capability of providing a maximum of 25 millimeters of $x$ and $y$ translation and 50 millimeters of $\mathrm{z}$ translation. $\mathrm{A}$ Huntington rotary platform (model \# VF-174-275) allowed the sample to be rotated through the full $360^{\circ}$. A pair of copper feedthroughs were welded to one of the mini flanges on the manipulator. These feedthroughs were used to attach W $5 \% \operatorname{Re} / \mathrm{W} 26 \% \mathrm{Re}$ thermocouple wires.

The actual sample holder was built in the building 62 machine shop under the supervision of Dan Colomb. It consisted of two hollow 0.25 inch copper tubes along with Pt / Pt 10\%Rh thermocouple feedthroughs. The copper tubes were sealed at the bottom and connected to 0.125 inch wide, 0.5 inch long tantalum rods. A short piece $(\sim 0.25$ inch) of twenty mil (.02 inch) rhenium wire was spotwelded to the inside surface of each of the tantalum rods. The molybdenum single crystal was in turn spotwelded between the two rhenium wires. Liquid nitrogen could be forced down the copper tubes allowing the sample to be cooled to $80-90 \mathrm{~K}$ in ten to fifteen minutes. Resistive heating of the crystal 


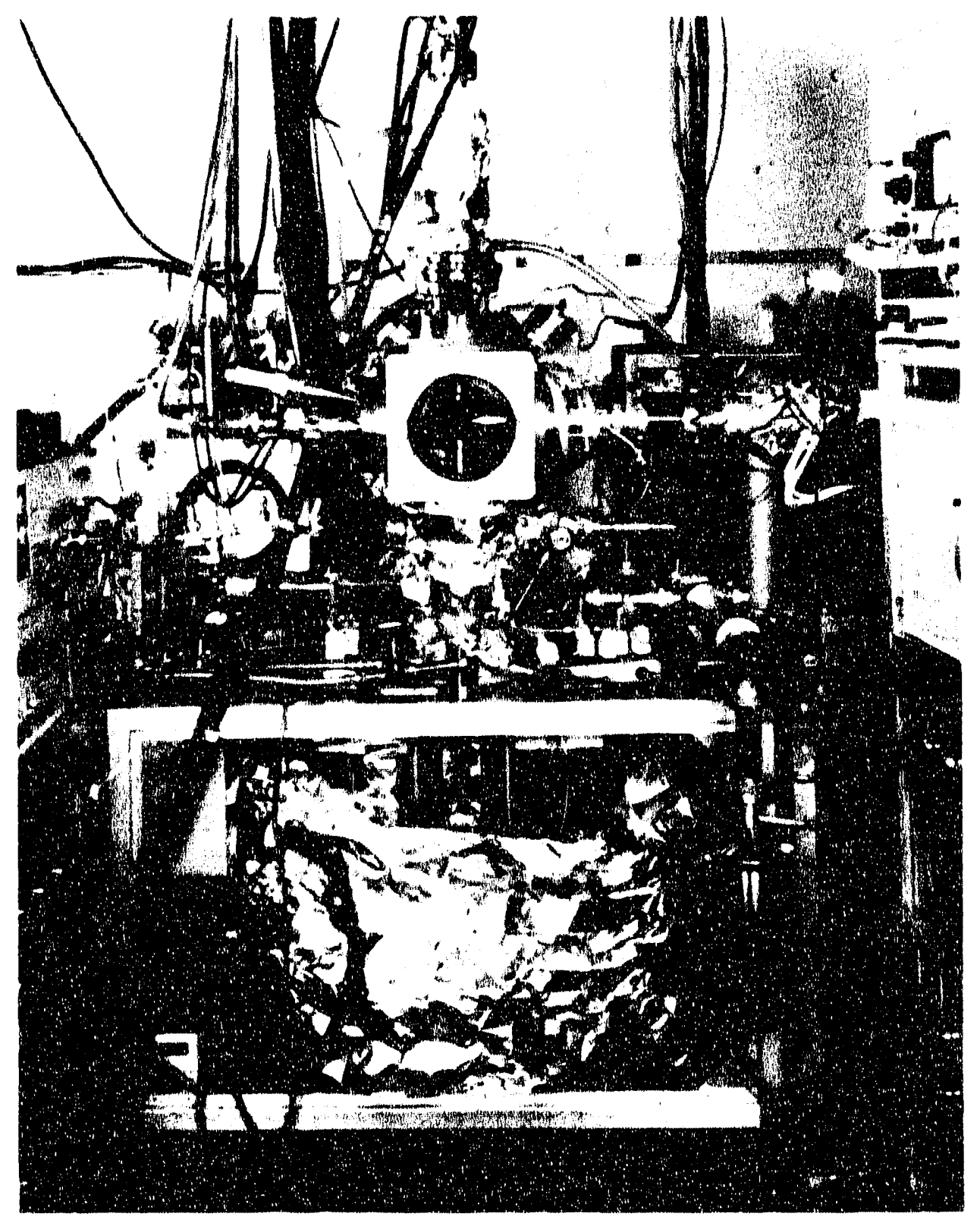

CBB $880-10134 A$

Figure 2.3: Photograph of ultra high vatuum system (taken in 1988). Since that time a new manipulator and cohalt source have been added. 

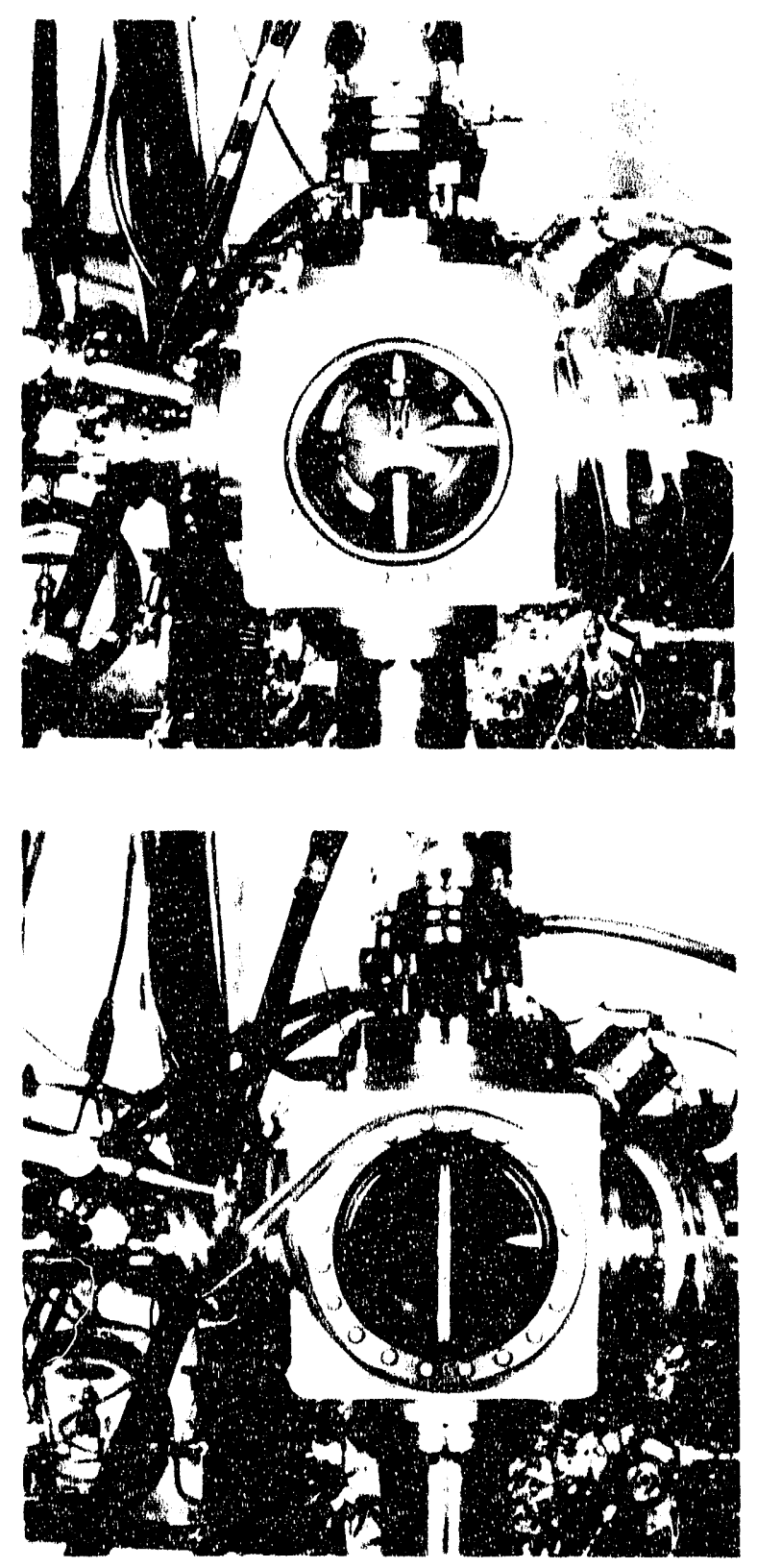

Figure 2.4: Photograph of UHV / high pressure cell apparatus used in the experiments. The high pressure cell is shown in both the open (upper picture) and closed (lower picture) positions. 
up to $1900 \mathrm{~K}$ was also accomplished using these tubes. These feedthroughs were welded into a stainless steel case. The bottom of the latter provided a sealing surface with the high pressure cell. The sample holder must be fully raised against a retainer welded to the ultra high vacuum side of the manipulator flange before forming a seal with the high pressure cell. Various components of the sample holder are shown in figure 2.5.

\subsubsection{High pressure cell and reaction loop}

The high pressure isolation cell was mounted on a linear motion feedthrough. It could be raised until a viton o-ring located in the groove on the top of the high pressure cell sealed by ultra high vacuum tight contact with the lower surface on the sample holder. In figure 2.6, a schematic of the high pressure cell and sealing surface of the manipulator is shown. Once sealed, the high pressure cell could be filled with an atmosphere of reactant gases to perform catalytic reactions. During such reactions, the background pressure varied from $1 \times 10^{-9}$ Torr (no detectable leak) to $>1 \times 10^{-7}$ Torr. Pressures $>1 \times 10^{-7}$ Torr were seen after several $(>50)$ closures of the high pressure cel'. and indicated that the viton o-ring needed to be replaced.

Two gas lines ran through the walls of the high pressure cell. These lines were connected to a closed reaction loop via ultra high vacuum compatible valves (Nupro). The loop was made of 0.25 inch stainless tubing. Included in the loop was a teflon circulation pump (Cole-Parmer) and a gas chromatography sampling valve. Periodically samples could be injected into the gas chromatograph (Perkin-Elmer Model 3920 B) to monitor the progress of the reaction.

The reaction loop was also connected to a mechanical pump and a two inch Varian oil diffusion pump. The low pressure regime $\left(10^{-3}<\mathrm{P}<1\right.$ Torr $)$ was monitored by a thermocouple gauge and the high pressure regime $(1<\mathrm{P}<780$ Torr $)$ was monitored by an 

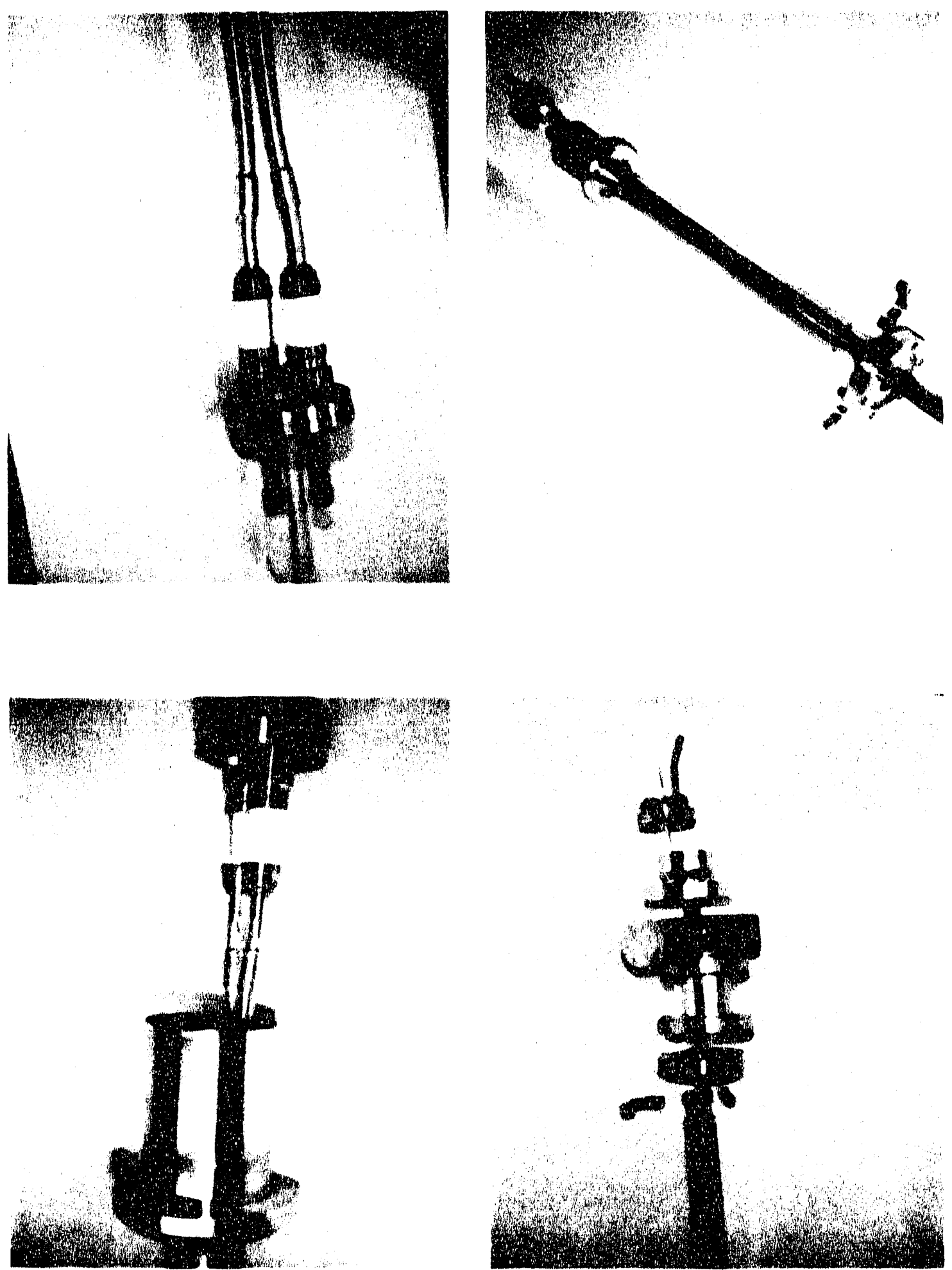

CBB $923-1800$

Figure 2.5: Photographs of the sample holder. 


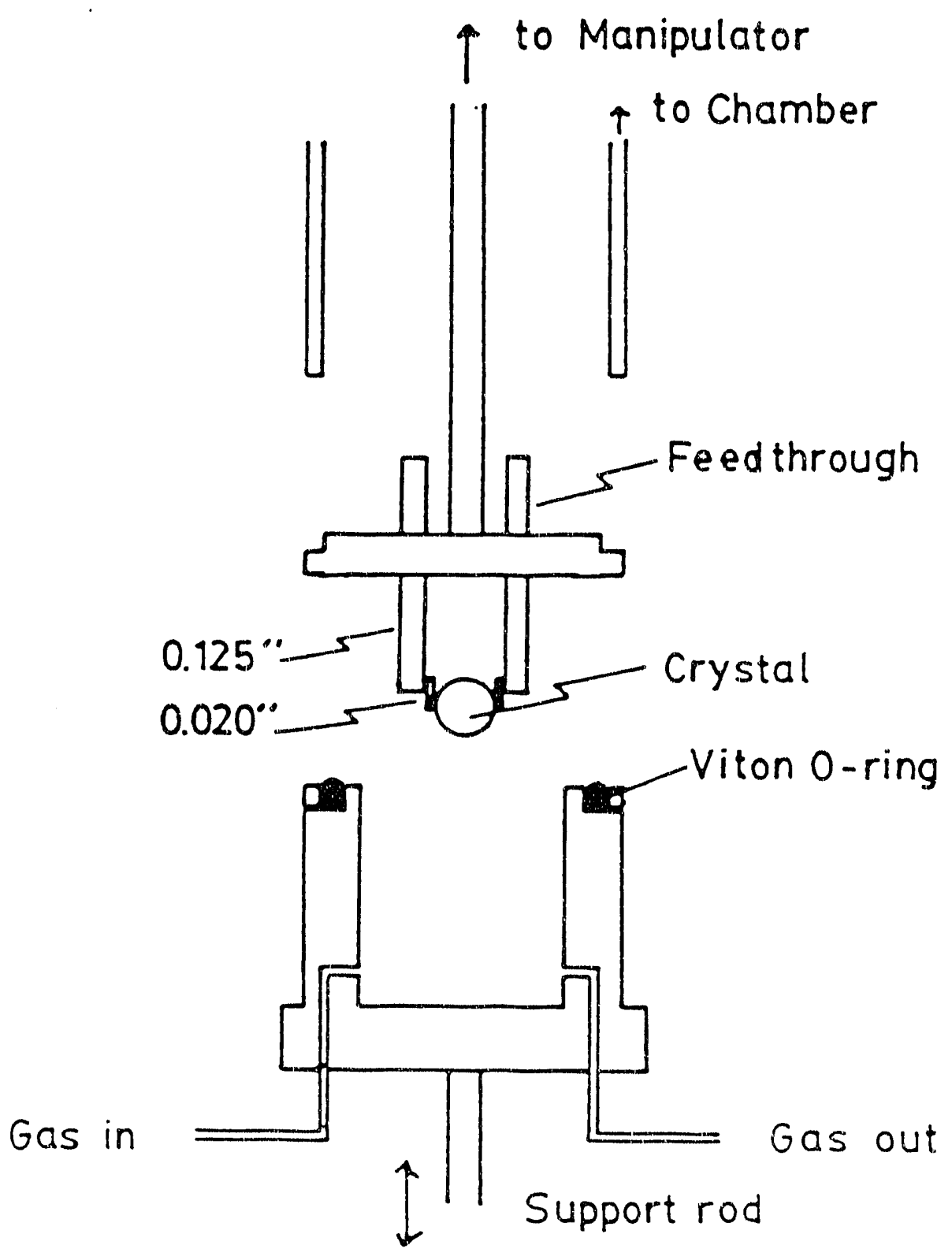

Figure 2.6: Schematic of high pressure cell and sealing surface of manipulator. 
absolute pressure gauge (Wallace \& Tiernan Model \# 62B-4D-0800).

\subsubsection{Pumps}

The chamber was constantly pumped by a Varian ion pump (model \# 923-0000) and a liquid nitrogen trapped six inch oil diffusion pump. In this diffusion pump, santovac oil was used. The ion pump originally had a pumping speed of 400 liters per second and the diffusion pump 1500 liters per second. The base pressure after a twenty four hour bake at $\sim 420 \mathrm{~K}$ was $1 \times 10^{-9}$ Torr. Several factors limited the attainment of a lower pressure. The titanium elements in the ion pump have not been changed for at least ten years and possibly much longer. (Because radioactive $\mathrm{C}^{35} \mathrm{~S}$ and ${ }^{14} \mathrm{C}_{2} \mathrm{H}_{4}$ were pumped ${ }^{3}$, caution is advised when replacing the elements). Also, thiophene is a sticky molecule and so adsorbs on the walls of the high pressure cell during reactions. Constant desorption from the walls place limits on the base pressure. The connection between the gas lines of the high pressure cell in the ultra high vacuum chamber and the external reaction loop was made with swage lok fittings. Swage lok connectors, though convenient, are not reliable at ultra high vacuum pressures. It would have been better if the design allowed the use of copper gaskets. The diffusion pump has only limited efficiency since it is separated from the chamber by a long, curved tube. This configuration limits the pumping efficiency. However, there is no alternative placement for the pump. Both this diffusion pump and the smaller pump used to evacuate the reaction loop were backed by mechanical pumps.

After the chamber had been opened to atmosphere, it was pumped down first using a mechanical pump equipped with a liquid nitrogen cooled trap. This trap served to prevent mechanical pump oil vapors from reaching the chamber. After roughing with the mechanical pump, the chamber was further pumped to 30 milliTorr using a liquid nitrogen cooled sorption pump. Once this pressure was attained, the diffusion purnp was opened. 
As a general practice, the gate valve separating the ion pump from the chamber was not opened until the chamber pressure was lowur than $1 \times 10^{-6}$ Torr. During high pressure $\left(10^{-7}\right.$ Torr) oxygen treatments, the ion pump was sealed from the chamber and only the diffusion pump was used.

\subsubsection{Sulfur source}

Sulfur was deposited using an electrochemical cell described elsewhere ${ }^{4}$ and shown schematically in figure 2.7. This electrochemical cell is composed of a solid silver iodide electrolyte sandwiched between silver and silver sulfide electrodes which form the cathode and anode respectively. When depositing sulfur, the cell is heated to about $420 \mathrm{~K}$ to increase the conductivity of the solid electrolyte and a voltage of $1.5 \mathrm{~V}$ is applied to cause electrolytic decomposition of silver sulfide to $\mathrm{S}_{2}$ and $\mathrm{Ag}$. The sulfur source was positioned such that the sulfur beam was condensed on the single crystal surface.

Ordered overlayers were prepared by first depositing saturation coverages of sulfur on molybdenum surfaces. Then the crystal was heated to various temperatures to desorb a fraction of sulfur and order the remaining sulfur. Ordered overlayers corresponding to coverages of one, three-quarters, two-thirds and half of a monolayer of sulfur were prepared by annealing at approximately $970 \mathrm{~K}, 1220 \mathrm{~K}, 1370 \mathrm{~K}$ and $1620 \mathrm{~K}$ respectively.

At these coverages, $c(2 \times 2),\left|\begin{array}{ll}2 & i \\ 1 & 1\end{array}\right|, c(4 \times 2)$ and $p(2 \times 1)$ structures are seen respectively.

\subsubsection{Cobalt source}

Initially cobalt was deposited on the crystal using a source which consisted of a one millimeter wide strip of five mil thick cobalt foil wrapped around twenty mil tantalum wire. The cobalt source was enclosed in a tantalum cone and equipped with a shutter which could 


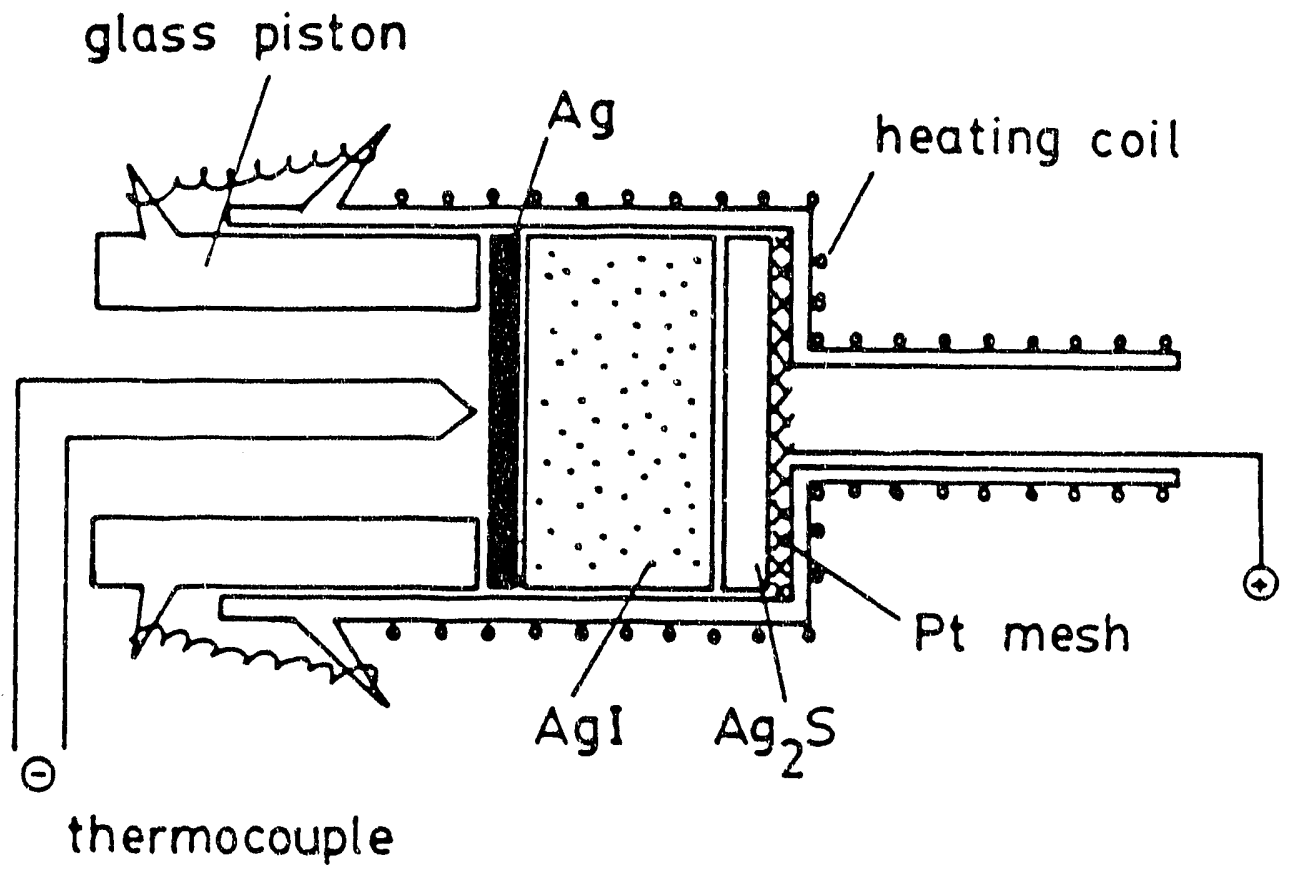

Figure 2.7: Schematic of electrochemical sulfur source. 
be opened to allow the vapor to reach the crystal. Cobalt was vaporized by heating the tantalum wire. However as this source was positioned about ten centimeters from the crystal, most of the cobalt was not deposited on the crystal but on other parts of the chamber. I had severe problems such as the electron gun shorted from metal deposition on the ceramics. Also cobalt was deposited on the LEED grids. In 1989, a new cobalt source was built by Robert Wright in the building 62 machine shop. This source was mounted on bellows and so could be positioned within a few millimeters of the crystal before opening the shutter. In this source cobalt ( $>99.95 \%$ purity) was melted into an alumina $(99.8 \%$ purity) crucible. Ten mil tantalum wire wrapped around the crucible was used to resistively heat it. A Pt / Pt 10\% Rh thermocouple mechanically fixed against the crucible provided an estimate of the temperature of the cobalt. The temp rature of the crucible was kept constant (within $\pm 5^{\circ}$ ) during a given deposition experiment.

\subsubsection{Retarding field analyzer}

A four grid retarding field analyzer (model \# 981-0005) purchased in 1962 was used for Auger spectroscopy and low energy electron diffraction. The screen of the retarding field analyzer was recoated several times during this thesis. The fourth grid was replaced. The electron gun used old style Varian filaments which are now only available from Spectra Mat. The techniques Auger spectroscopy and low energy electron diffraction are described in sections 2.3 .1 and 2.3 .2 respectively.

\subsubsection{Mass Spectrometer}

A UTI mass spectrometer (model $100 \mathrm{C}$ ) was employed. The ionizer was enclosed in a gold collimator. The orifice of the collimator was less than five millimeters in 
diameter, which is smaller than the diameter of the samples used. The mass spectrometer was mounted on bellows and during thermal desorption experiments, the orifice of the collimator was brought to within three millimeters of the centers of the single crystals to minimize the detection of gases desorbing from the support wires or other parts of the manipulator. It would have been desirable to differentially pump the mass spectrometer during such thermal desorption experiments. However, this capability was not available in this system. Thoriated iridium filaments were used. The electron multiplier was replaced in 1989 and at the end of this work a channeltron voltage of $1640 \mathrm{~V}$ provided a gain greater than $10^{4}$.

\subsection{Surface analytical techniques}

The techniques used to characterize the elemental composition and structures present on the surface are described below. These are the techniques of Auger electron spectroscopy and low energy electron diffraction respectively. Thermal desorption spectrometry was also used and is described in Section 2.3.3. In all three cases, a general discussion of the technique is followed by specific information on the parameters used in these experiments. For all of the techniques, references in which they are described in detail are provided.

\subsubsection{Auger electron spectroscopy}

Auger spectroscopy $y^{2,5}$ is probably the most common of the electron based techniques for elemental surface analysis. A core hole in an atom can be created by bombarding the atom with electrons that have higher kinetic energies than the particular 
core level or through excitation with sufficiently energetic $\mathbf{x}$-ray radialion. Such an ionized atom will return to the ground state by one of two ways. An electron from a higher level can be demoted to the core level and the energy released by this process emitted as a photon with a characteristic energy (wavelength). The alternative de-excitation pathway is a radiationless one. In this pathway, an electron from a higher level is again demoted to the core level. However the energy released is given to a second electron. This second electron, the Auger electron, leaves the atom with a characteristic kinetic energy. With increasing core level energy, the Auger electron yields decrease and the probability for $x$-ray radiation increases. In Auger spectroscopy of solids, the initial kinetic energy of the Auger electron is decreased by the work function of the material. In addition, if both the electron filling the core hole and leaving the solid are emitted from the valence band, then the lineshape of the energy distribution is influenced by the band structure of the solid. The kinetic energy of Auger electrons is given below.

$$
\begin{aligned}
& E_{x y z}=E_{x}-E_{y}-E_{z}-\varepsilon_{z}-e \varphi \\
& \text { where } \\
& E_{x y z}=\text { kinetic energy of the Auger electron } \\
& E_{x}=\text { binding energy of the core level electron } \\
& E_{y}=\text { binding energy of the electron that is demoted to the core level } E_{\mathrm{x}} \\
& E_{\mathrm{z}}=\text { binding energy of the Auger electron (that leaves the solid) in the bound state } \\
& \varepsilon_{\mathrm{z}}=\text { correction to account for the presence of a hole in the } Y \text { shell (ie. increased } \\
& \varphi \text { positive potential) when the Auger electron is removed from the } Z \text { shell } \\
& \varphi=\text { work function of the analyzer }
\end{aligned}
$$

The Auger process is shown schematically in figure 2.8 . The kinetic energy of Auger electrons depends on the energy levels of the element from which they are derived 


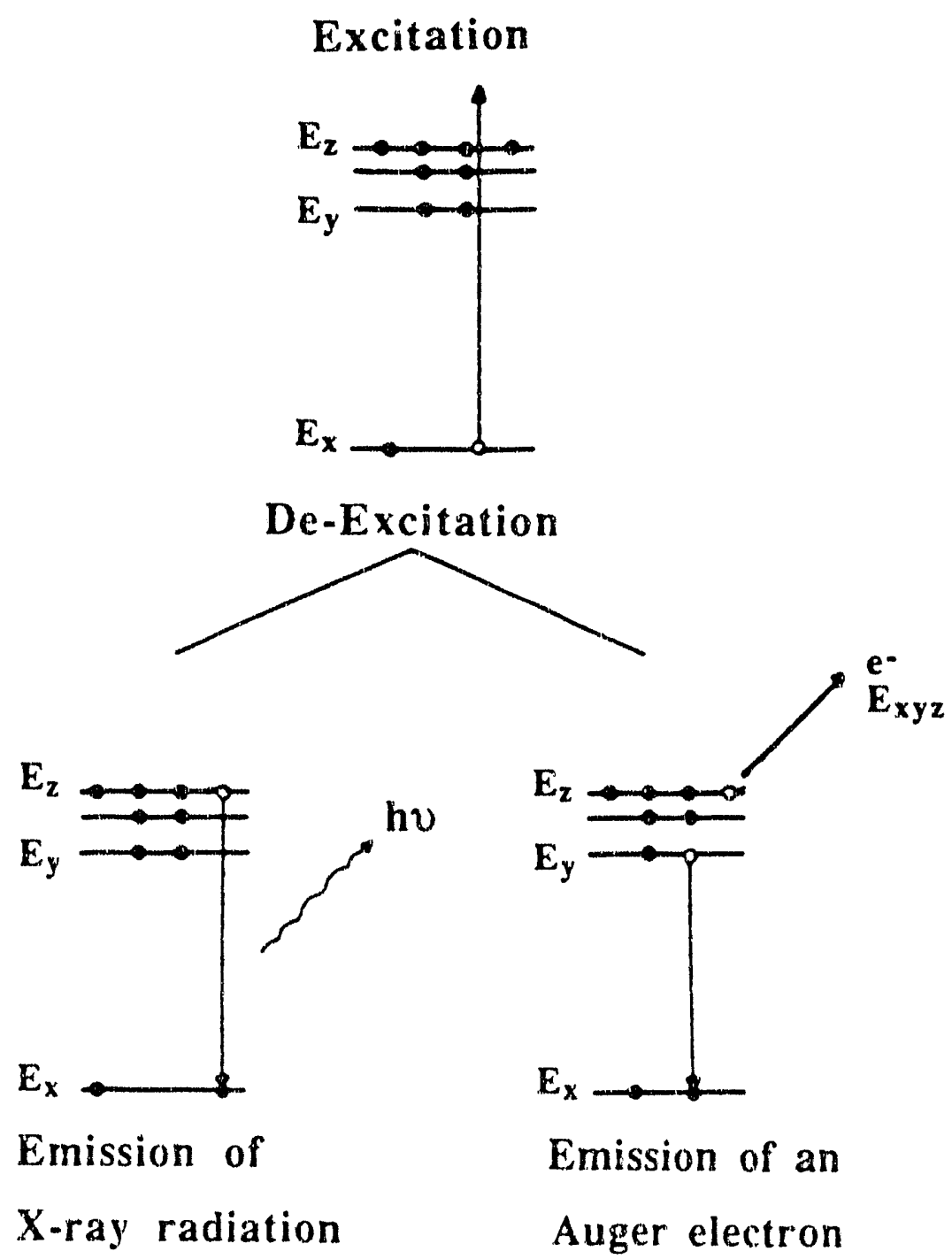

Figure 2.8: Schematic of Auger process. 
and is independent of the means of initial excitation. Since the binding energies of core electrons are characteristic of the atomic identity, measuring the kinetic energy of Auger electrons provides surface elemental information. Energies of Auger electrons for all elements except hydrogen and helium are documented ${ }^{6}$. Hydrogen and helium do not have enough electrons to undergo the Auger process. The kinetic energies of the Auger electrons typically used range from 30 to $1000 \mathrm{eV}$. Electrons with these kinetic energies interact strongly with atoms in a solid and so these electrons have short (2 - 3 atomic layers) mean free paths in solids. This accounts for the surface sensitivity of Auger spectroscopy. As shown in figure 2.1, Auger electrons are present on a large background of secondary electrons. To better reveal their presence, the signal derivative $\mathrm{dN}(\mathrm{E}) / \mathrm{dE}$ is taken.

The kinetic energy of Auger electrons were analyzed using a four grid retarding field analyzer. A schematic of such an analyzer and signal amplification circuit is shown in figure 2.9. In operation, a grazing incidence ( $70^{\circ}$ from the surface normal) electron beam is impinged onto the grounded sample and electrons are backscattered. A variable negative retarding voltage is applied to the second and third grids which are connected to each other. These retarding grids are positioned between the first and fourth grids, both of which are grounded. Located behind the fourth grid is a positively charged fluorescent screen. The backscattered electrons are accelerated towards the screen. However in order to reach the screen and be detected, they must have kinetic energies greater than the negative potential of the retarding grids. The collector current is a function of the retarding potential and is proportional to the number of electrons emitted from the crystal that have energies greater than the negative retarding potential. As mentioned previously, the energy distribution is differentiated to enhance the Auger signal. This is done electronically by superimposing a small amplitude oscillating voltage with frequency $\omega$ on the retarding voltage. It has been shown that when this is done, the second derivative of the signal is proportional to the 


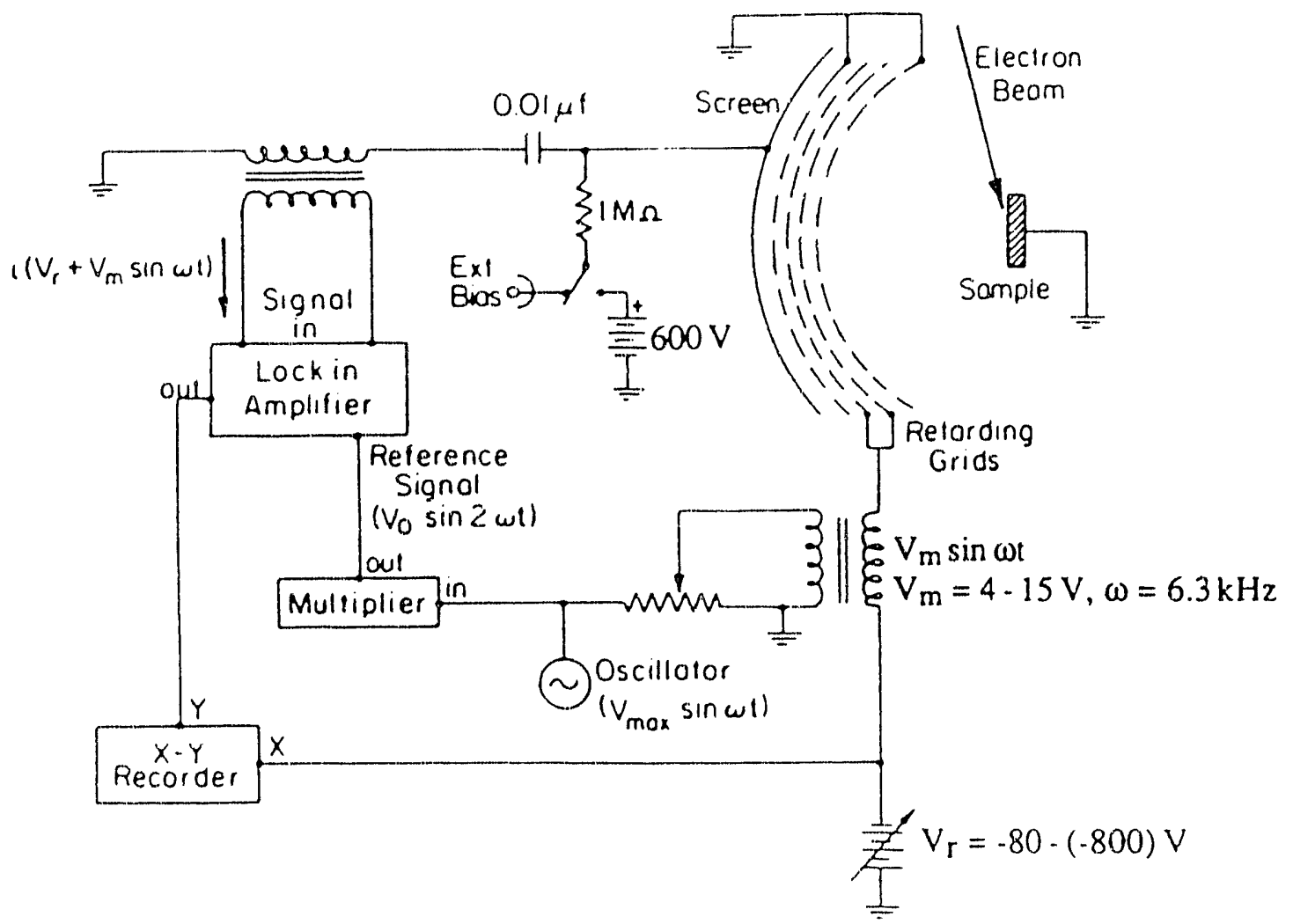

Figure 2.9: Schematic of analyzer and signal amplification circuit used in the Auger experiment. 
(a)

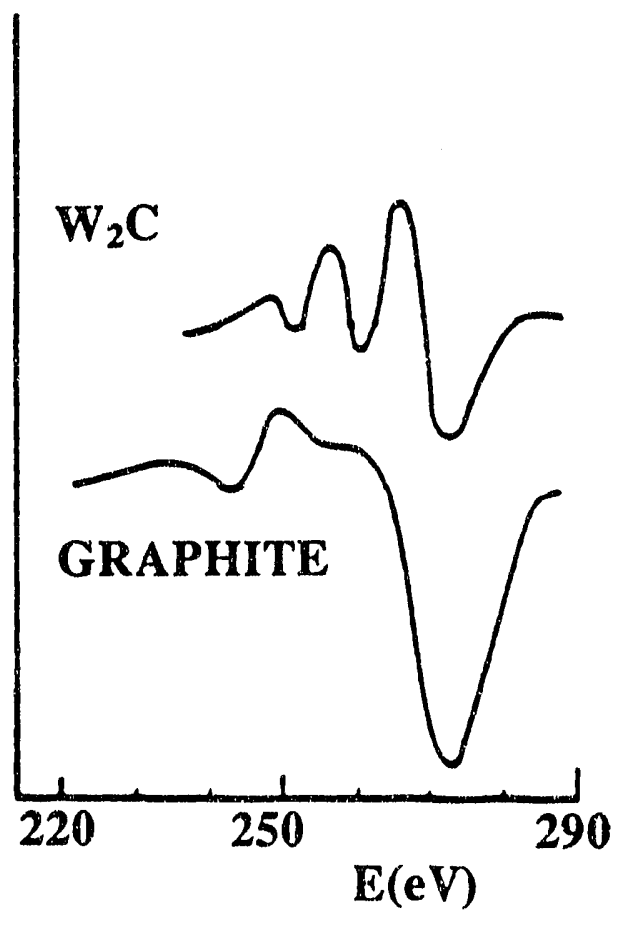

(b)

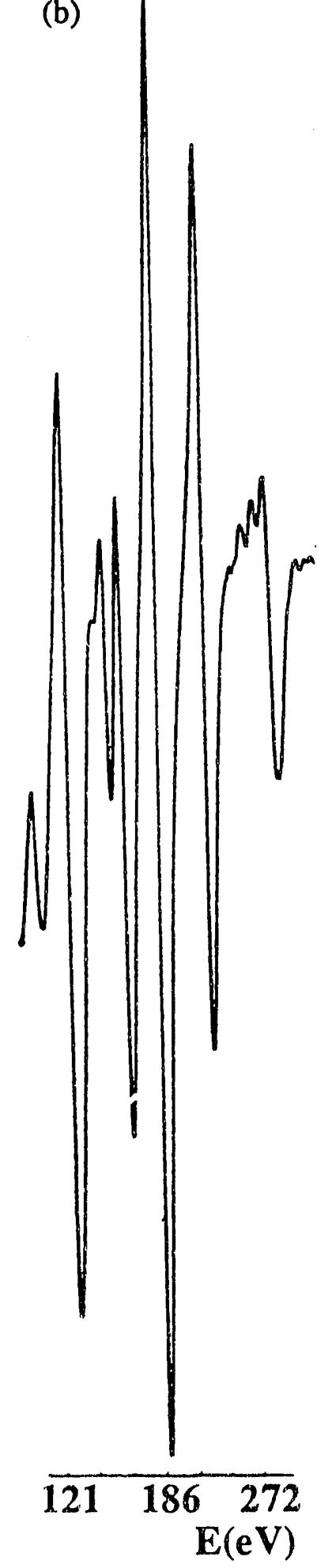

Figure 2.10: Carbon Auger lineshapes for (a) a graphite overlayer on tungsten and $\mathrm{W}_{2} \mathrm{C}$ and (b) a carbidic overlayer on molybdenum. 
component of the collector current with frequency $2 \omega$. Increasing the oscillating voltage increases the signal intensity but decreases the resolution. In this work, electrons with a primary energy of $2 \mathrm{KeV}$ were used along with a crystal current of $\sim 10 \mu \mathrm{A}$. The retarding voltage on the second and third grids was ramped between -80 and $-800 \mathrm{eV}$. The accelerating voltage on the screen was $+600 \mathrm{eV}$. When recording different spectra, the oscillating voltage was varied between 4 and $15 \mathrm{eV}$.

Auger spectroscopy was used to check the cleanliness of the molybdenum single crystals and to estimate the coverages of sulfur, cobalt and carbon. Determination of sulfur coverages is aided by the high cross section of sulfur and the appearance of ordered structures at different sulfur coverages. The determination of cobalt coverages was more difficult. The sensitivity of the retarding field analyzer decreases with increasing kinetic energy of electrons being detected. Therefore the sensitivity to the cobalt Auger electrons $(650-775 \mathrm{eV})$ was considerably less than for the $148 \mathrm{eV}$ sulfur or $186 \mathrm{eV}$ molybdenum Auger electrons detected. Auger spectroscopy also aided in the determination of the type of adsorbed carbon. The carbon KLL Auger transitions involve valence electrons. As mentioned earlier, in such cases the Auger lineshape can help in the determination of the atomic environment. In the case of carbon, the lineshape can help determine whether carbidic or graphitic carbon is present. The different Auger lineshapes of graphitic and carbidic overlayers on tungsten are shown and compared to the lineshapes observed for carbon overlayers on molybdenum in this work (figure 2.10).

\subsubsection{Low energy electron diffraction}

Electron diffraction techniques have their foundation in the wavelike behavior of electrons and their corresponding de Broglie wavelength $\left(\lambda=h / m_{e} v\right)$. De Broglie postulated almost seventy years ago, that moving objects have associated wavelengths $(\lambda)$ 
characteristic of their mass ( $m$ ) and velocity (v). In the equation above $h$ is Planck's constant $\left(6.63 \times 10^{-34} \mathrm{Js}\right)$. The small magnitude of Planck's constant ensures that the wavelengths associated with macroscopic objects are extremely small. For microscopic particles, such as electrons, the wavelengths are of the same order of magnitude as atomic distances. Hence by diffraction of electron waves scattered from surfaces, these electrons can be used as probes of atomic distances. Electrons with energies varying between 10 and $500 \mathrm{eV}$ have wavelengths $\left(\lambda(\AA)=\mathrm{h} /(2 \mathrm{mE}(\mathrm{eV}))^{1 / 2}\right.$ varying between 3.9 and $0.64 \AA$. These wavelengths span the range of most interatomic distances. By fortunate coincidence, these same electrons that can be used to probe atomic distances, have mean free paths of only a few atomic layers in solids (figure 2.2). This ensures that the structural information that they carry is derived from the surface region. Between 1925 - 1927, Davisson and Germer $^{7}$ at Bell Laboratories provided experimental proof of the wave nature of electrons, when they observed electron diffraction by nickel crystals. Today, diffraction of low energy electrons is routinely used to determine the symmetry and size of periodic surface structures. Surface crystallography is also used, though less routinely to determine surface bond lengths, bond angles and adsorption sites.

In low energy electron diffraction ${ }^{5,8}$, a monoenergetic (within $.3 \mathrm{eV}$ ) beam of electrons is impinged onto a clean or adsorbate covered single crystal surface. Generally, the sample is positioned normal to the electron beam. Less than $\sim 1 \%$ of the electrons are elastically backscattered. The angles of emergence of the diffracted beams are determined by energy conservation $\left(E_{\text {initial }}=E_{\text {final }}\right.$ ) and conservation of linear momentum parallel to the surface. This is shown schematically in figure 2.11 . In the case of the (00) beam, the parallel momentum before and after diffractive scattering are exactly equal. For higher order beams, the parallel momentum component of the wavevector after diffractive scattering is the vector sum of the incident parallel momentum and any surface reciprocal lattice vector (eq. 1). Each diffracted beam therefore corresponds to a different reciprocal 


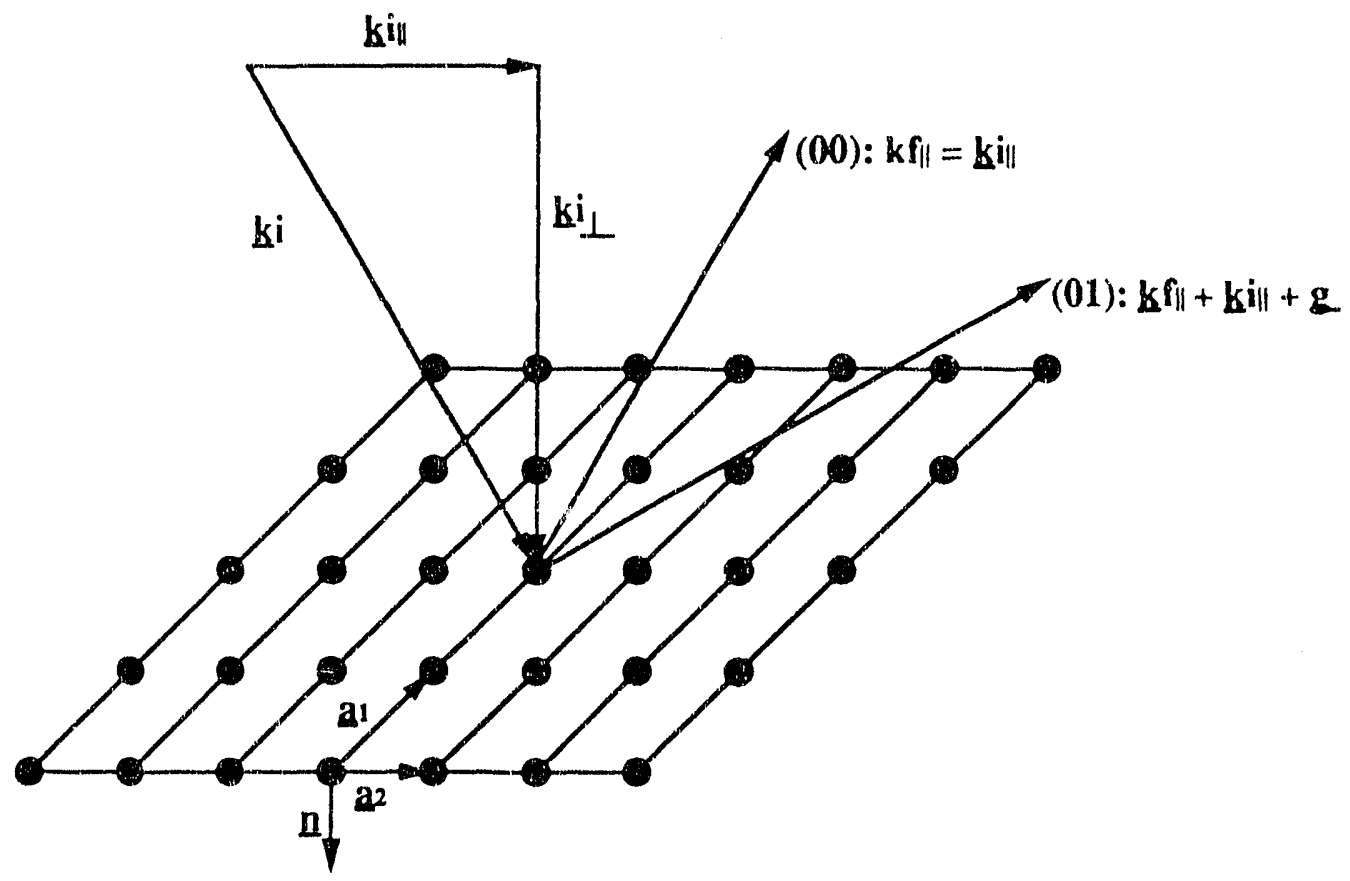

Figure 2.11: Conservation of linear momentum parallel to the surface in a LEED experiment. 
lattice vector.

$$
\text { eq. } \begin{aligned}
1 \quad \underline{k}_{f \mid} & =\underline{k}_{i \|}+g_{\|} \\
& \text {where } \\
\underline{k}_{f \|} & =\text { parallel momentum component of diffracted wave vector } \\
\underline{\mathbf{k}}_{i \|} & =\text { parallel momentum component of incident wave vector } \\
\mathrm{g}_{\|} & =\text {any surface reciprocal lattice vector }
\end{aligned}
$$

Reciprocal lattice vectors have units of length ${ }^{-1}$ and are defined by the relationship below.

$$
\begin{aligned}
\text { eq. } 2 \quad \underline{b}_{j} \cdot \underline{a}_{j} & =2 \pi \delta_{i j} \\
& \text { where } \\
\delta_{i j} & =1 \text { if } \mathrm{i}=\mathrm{j}, \quad 0 \text { if } \mathrm{i} \neq \mathrm{j} \\
\mathbf{a}_{1}, \underline{\mathrm{a}}_{2} & =\text { two dimensional basis vectors of the surface lattice } \\
\underline{\mathrm{b}}_{1}, \underline{\mathrm{b}}_{2} & =\text { two dimensional reciprocal lattice vectors }
\end{aligned}
$$

The unit cells of crystals have both a real space crystal lattice and a reciprocal lattice associated with the structure. The diffraction pattern is an image of the reciprocal lattice. $\mathrm{g}_{\| 1}$ is a reciprocal lattice vector and as such can be written as a linear combination of the appropriate basis vectors.

$$
\text { eq. } 3 \quad g_{\|}=h \underline{b}_{1}+1 \underline{b} \quad h, I \text { integers }
$$

$$
\begin{array}{rll}
\text { by definition of eq. } 2 & \mathrm{~g}_{\|} \cdot \mathrm{a}_{1}=2 \pi \mathrm{h} & \text { eq. } 4 \mathrm{a} \\
& \mathrm{g}_{\|} \cdot \mathrm{a}_{2}=2 \pi \mathrm{l} & \text { eq. } 4 \mathrm{~b} \\
\text { and from eq. } 1 & \mathrm{~g}_{\|}=\underline{\mathrm{k}}_{f_{\|}}-\underline{\mathrm{k}}_{\mathrm{i}} & \text { eq. } 5 \\
& \underline{\mathrm{k}}_{\mathrm{i}}=\underline{\mathrm{k}} \sin \varphi_{\mathrm{i}} & \text { (figure 2.11) }
\end{array}
$$




$$
\begin{aligned}
\text { by definition } & & |\underline{k}|=2 \pi / \lambda \\
\text { therefore } & & \left|k_{i \|}\right|=2 \pi / \lambda \sin \varphi_{i} \\
\text { and similarly } & & \left|k_{f_{\|}}\right|=2 \pi / \lambda \sin \varphi_{f} \\
\text { therefore } & & \left|\underline{g}_{\|}\right|=2 \pi / \lambda\left(\sin \varphi_{f}-\sin \varphi_{i}\right)
\end{aligned}
$$

As stated previously, low energy electron diffraction experiments are generally performed with the primary electron beam normal to the crystal surface ( $\left.\sin \varphi_{i}=0\right)$. Substituting this definition of $g_{\|}$into eq. $4 a$ under normal incidence conditions yields $\left(a_{1} \sin \varphi_{f}=\lambda h\right)$, the well known Bragg requirement for constructive interference of waves scattered from periodic surfaces .

Since it is the energy $\left(E=h^{2} / 2 m \lambda^{2}, m=9.11 \times 10^{-28} \mathrm{~g}\right)$ of these electrons which is measured, this is substituted for the wavelength in the equation below.

$$
\sin \varphi_{\mathrm{f}}=\frac{1}{\mathrm{a}_{1}(\AA)}\left(\frac{150}{\mathrm{E}(\mathrm{eV})}\right)^{1 / 2}
$$

This equation is valid for a one dimensional periodic lattice. An arrangement of lattice points that are periodic in two dimensions can be considered as an assembly of parallel rows of scatterers. Each parallel group of rows can be assigned a direction [h' $k$ ']. The distance between parallel rows is $d_{h '}$ ' . For a periodic two dimensional lattice, interference maxima are seen in directions given below.

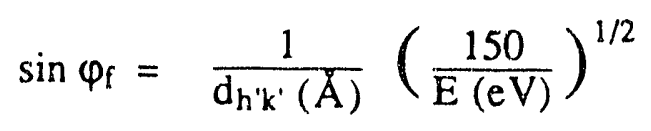

It can be seen that by increasing the electron energy, the angle $\varphi$ at which a given beam appears decreases. Therefore by increasing the energy, the beams move towards the (00) beam and additional beams become visible. The (00) beam corresponds to direct reflection 


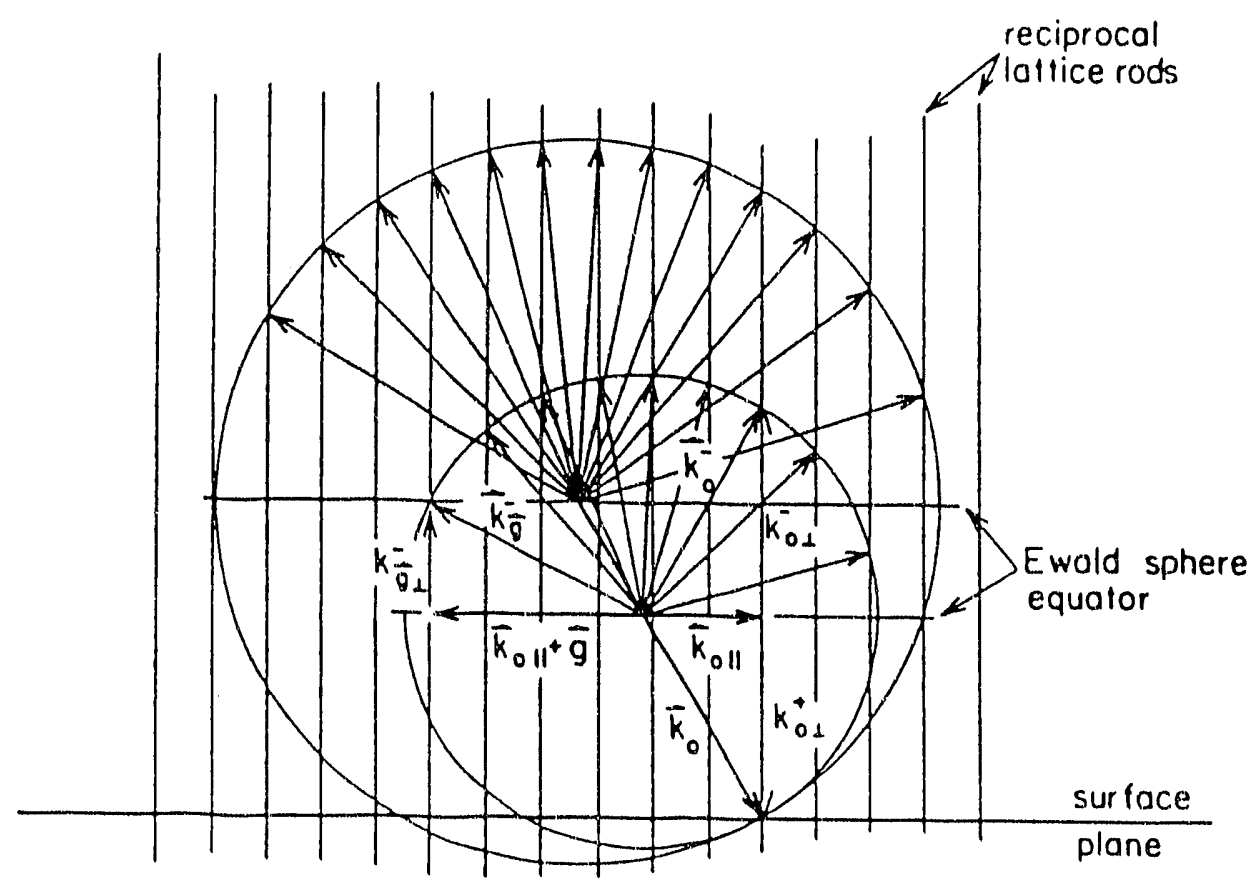

Figure 2.12: Ewald sphere construction for low energy electron diffraction from surfaces. $\underline{k}_{\mathrm{o}}=$ initial wave vector, $\underline{\mathrm{k}}_{\mathrm{g}}=$ final wave vector and $\mathrm{g}=$ any surface reciprocal vector. 
and does not change position with energy.

Alternatively, the angle of emergence of the diffracted beams can be determined by the Ewald sphere construction (figure 2.12). In the real space two dimensional crystal lattice, there are periodic rows of lattice points. In reciprocal space, these periodic rows of points become periodic rows of rods perpendicular to the crystal surface. These rods are shown schematically in this construction. The magnitude of the incident momentum wave vector $(\underline{k})$ is determined by the energy $\left(\lambda \approx 1 / \underline{k}, \underline{k} \approx(2 \mathrm{mE}(\mathrm{eV}))^{1 / 2} / \mathrm{h}\right)$. In the Ewald construction, a sphere of radius $k$ is drawn appropriate for the energy. This ensures that electron energy is conserved. The sphere is located such that it intercepts the (00) rod at we surface. Except at the lowest energies, the Ewald sphere will intercept other reciprocal lattice rods. From the figure, it is seen that these intersection points occur only where $\underline{\mathrm{k}}_{f_{||}}=\underline{\mathrm{k}}_{i||}+\mathrm{g}$. The diffracted beams emerge in the direction of $\underline{\mathrm{k}}_{f_{\| \mid}}$as shown. As the energy is increased, the wave vector $\mathbf{k}$ increase in magnitude and the center of the Ewald sphere shifts. As is also shown in figure 2.12, the angle of emergence of any given higher order beam decreases with increasing energy. Therefore with increasing energy, a given higher order beam will move towards the $(00)$ beam. As the radii of the Ewald sphere increases (with increasing energy), a greater number of reciprocal lattice rods will be intercepted and hence a greater number of beams will become visible.

From the observed diffraction pattern, the relative direction and length of the real space surface unit vectors $\left(\mathbf{a}_{1}, \underline{a}_{2}\right)$ can be determined using the foilowing relationships.

$$
\begin{aligned}
& \underline{\mathrm{b}}_{1} \cdot \underline{\mathrm{a}}_{2}=\underline{\mathrm{b}}_{2} \cdot \underline{\mathrm{a}}_{1}=0 \quad \text { and } \\
& \underline{\mathrm{b}}_{1} \cdot \underline{\mathrm{a}}_{1}=\underline{\mathrm{b}}_{2} \cdot \underline{\mathrm{a}}_{2}=2 \pi
\end{aligned}
$$

The presence of ordered overlayers increases the size of the primitive unit cell relative to the clean surface. This increased periodicity causes the observation of "fractional order" beams 


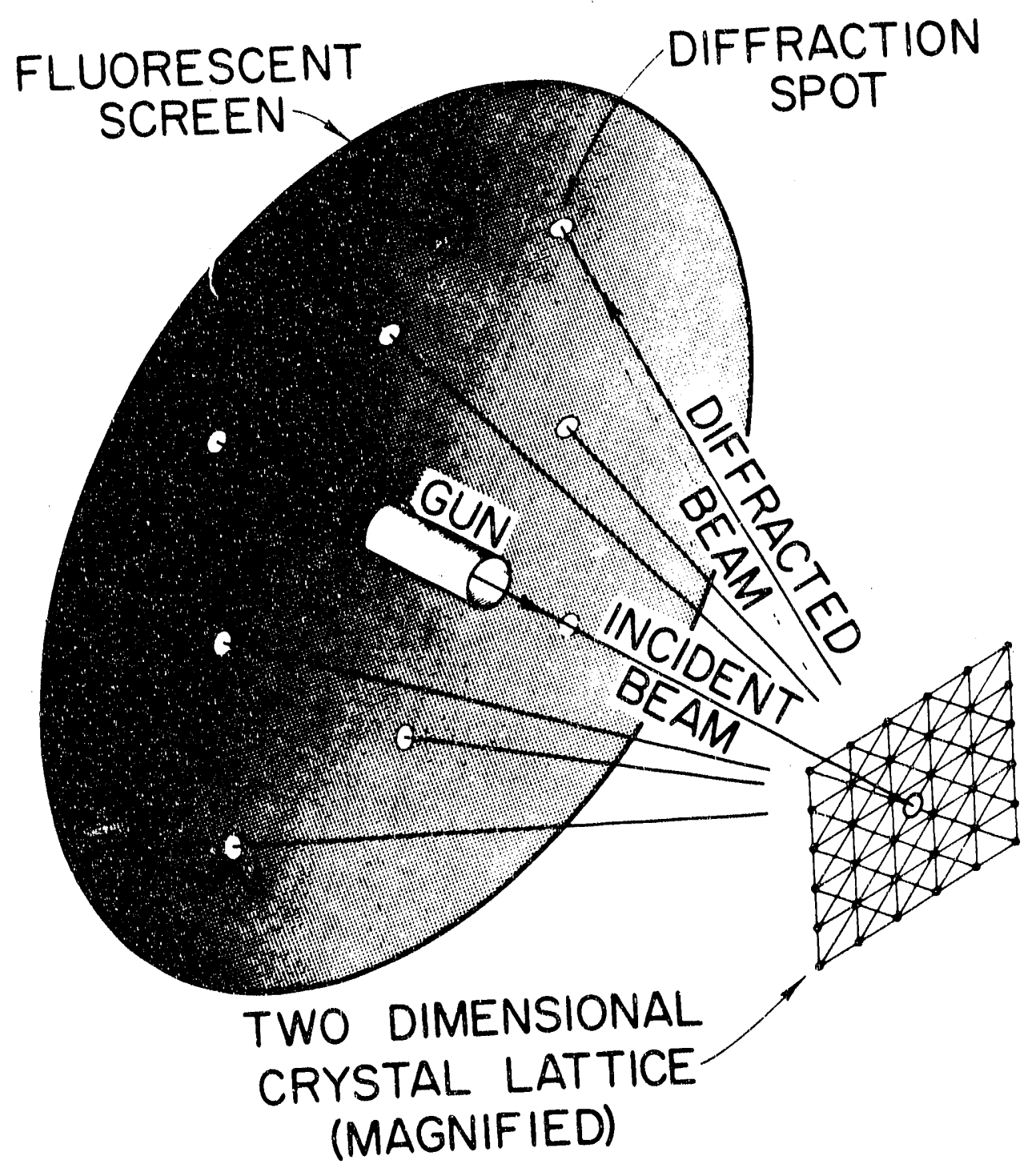

XBB 708-3583

Figure 2.13: Schematic of LEED experiment. 
between the substrate spots. The symmetry and size of the structure relative to the clean surface can be determined simply by observation of the diffraction pattern. However, the derivation of atomic positions from the diffraction pattern is not straightforward because the elastic scattering cross section for low energy electron-atom collisions is very large $\left(\sim 1 \AA^{2}\right)$. Therefore there is a great probability that electrons are diffracted more than once in the first few atomic layers of the crystal. This multiple scattering sends electrons in diffraction directions different from their original path. Multiple scattering does not change the positions of the diffraction beams. It does however affect the intensity of these beams. Nevertheless, by analyzing the energy dependence of the intensity of the diffraction spots and comparing these with theoretical simulations for model structures, atomic configurations for the near surface region can be determined with high precision. These calculations can only be performed on huge modern computers and are very time consuming. They therefore have been applied to less than three hundred different structures.

In this work, low energy electron diffraction was used to determine the symmetry and size of ordered overlayer structures relative to the clean surface and the direction and height of steps on vicinal surfaces. Electrons from the integral gun (of the retarding field analyzer) were bombarded onto the grounded crystal. The electron energies used varied between 50 and $180 \mathrm{eV}$. The first grid was grounded to produce a field free region between the crystal and the retarding field analyzer. A slightly $(<5 \mathrm{eV})$ lower negative voltage than the primary energy of the electrons was applied to the connected second and third grids. This retarding grid ensured that only the elastically scattered primary electrons reached the detector. The fourth grid was again grounded. A high positive voltage $(5-7 \mathrm{KeV})$ on the fluorescent screen accelerated the electrons and excited the screen phosphor upon electron impact. The screen was coated with silver activated zinc sulfide 9 . Applying a high voltage, causes this material to fluoresce with a blue color and show 
brighter spots at points where the diffracted beams intercept the screen. The experime it is shown schematically in figure 2.13 .

\subsubsection{Thermal desorption spectrometry}

A measurement of the remperature range at which molecules desorb from surfaces can provide an estimate of the strength of the molecule-surface chemical bond. Gas molecules with average kinetic energies $E_{k}$, that strike a solid surface can either bounce away from the surface or bond to it. In order to stick, striking molecules must lose energy to the surface that is at least equal to their kinetic energy. This energy can be lost by exciting surface phonons or plasmons. This loss serves to bring the molecule into equilibrium with the surface. As molecules $\left(X_{2}\right)$ approach the surface $(M)$, their potential energy will vary in a manner shown schematically in figure 2.14 . The molecules can lose potential energy and reside in a shallow energy minimum at a distance that is approximately equal to the sum of the Van der Waal radii of the surface atom and adsorbate molecule. In the case of figure $2.14(a)^{10}$, the physisorbed molecule $\mathrm{X}_{2}$ can overcome a srnall activation jarrier to dissociatively chemisorb onto the surface. Alternatively, $X_{2}$ might not physisorb in that precursor state, but directly chemisorb onto the surface forming two $\mathrm{M}-\mathrm{X}$ bonds. By comparison, in the case of figure 2.14(b), the activation energy to chemisorption is significant. Without the energy to overcome such a barrier, the molecule will stay in the physisorbed state. The binding energy of physisorbed molecules is generally less than $15 \mathrm{kcal} / \mathrm{mol}$.

The two dimensional phase approximation ${ }^{11}$ suggests that chernisorbed molecules will diffuse on metal surfaces and sample many adsorption sites before they desorb. In such cases, the molecules will attain their equilibrium distribution on different surface sites.

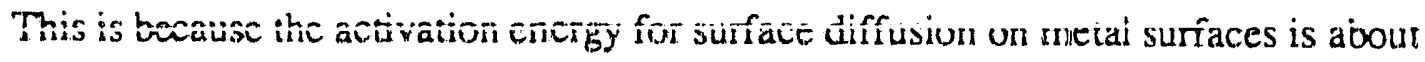




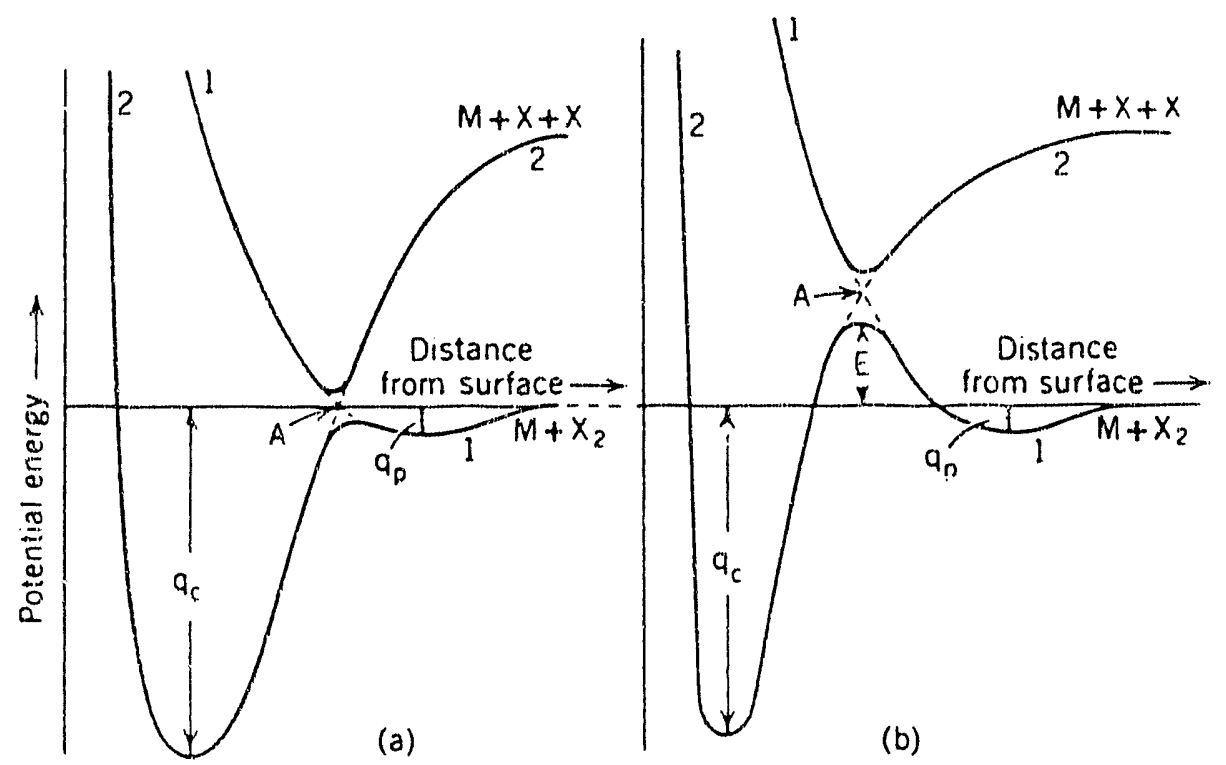

Figure 2.14: One-dimensional potential energy curves for (a) non activated dissociated adsorption through a physisorbed state and (b) activated chemisorption. 
$10-15 \%$ of the activation energy for desorption. Because of the extensive delocalization of metallic electrons, the comugation of the density of states around the atomic cores is very small. This makes it very easy for an adsorbate to diffuse from one metal atom to another. By comparison, on semiconductor surfaces, where the corrugation of the density of states close to the Fermi level is significant, the activation energies for surface diffusion and desorption are comparable. To desorb from a surface, molecules must acquire energy at least equal to the activation energy of desorption $\left(E_{d}\right)$. In the case of figure 2.14(a), the activation energy for desorption from a particular surface site can be approximated to be equal to the activation energy of adsorption on that same site. On clean metal surfaces this is often the case since adsorption is generally a non activated process ${ }^{12}$.

Thermal desorption spectrometry ${ }^{12,13}$ allows the estimation of the activation energies for desorption. It is possible for molecules to diffuse from their adsorption site to another site before desorbing. Therefore thermal desorption experiments do not necessarily provide information about adsorption sites and their energies. In the experiment, a surface is given a timed exposure to a known gas. After the exposure, the temperature of the sample is increased linearly with time. The desorption of molecules and their decomposition fragments are detected with a mass spectrometer. The relationship between the rate of desorption $(R)$, activation energy for desorption $\left(E_{d}\right)$, surface temperature $(T)$ and surface coverage $(\theta)$ is shown below.

$$
\begin{aligned}
& R=A \theta^{n} v_{n} \exp \left(-E_{d} / R T\right) \\
& \text { where } \\
& A=\text { surface area } \\
& n=\text { reaction order } \\
& v_{n}=\text { pre-exponential factor }
\end{aligned}
$$


As the sample temperature is raised, the desorption rate increases because of the exponential term. However as the coverage decreases, the $\theta^{n}$ term will become small, causing the rate to decrease with further increase in temperature. This change in desorption rate will show itself as a peak in the mass spectrum. It has been shown that when a constant heating rate ( $\beta$ ) is used, the activation energy of desorption $\left(E_{d}\right)$ can be related to the temperature at the rate of maximum desorption $\left(T_{p}\right)$. The exact relationship depends on the desorption order. The equations below show the relationship for first and second order desorption kinetics.

$$
\begin{array}{ll}
\frac{E_{d_{1}}}{R T_{p}^{2}}=\frac{v_{1}}{\beta} e^{\left(-E_{d_{1}} / R T_{p}\right)} & n=1 . \\
\frac{E_{d 2}}{R T_{p}^{2}}=\frac{v_{2} \theta}{\beta} e^{\left(-E_{d 2} / R T_{p}\right)} & n=2 .
\end{array}
$$

The activation energy for desorption and the pre-exponential factor can be determined by performing thermal desorption using several heating rates varying over at least two orders of magnitude. However, in this thesis, as is commonly done for first order desorption kinetics, a pre-exponential factor of $10^{13} \mathrm{sec}^{-1}$ is assumed. Using this assumption, the activation energy of desorption was estimated using Redhead's plot of the variation of first order activation energies of desorption with the temperature at maximum rates of desorption ${ }^{13}$. This plot is shown in figure 2.15. In generating this plot, it was assumed that the activation energy of desorption and the pre-exponestial factor are independent of the surface coverage.

In this work, thermal desorption spectrometry was performed using the set up described in section 2.2.7. A schematic of the desorption experiment is shown in figure 2.16. Gases were dosed using pressures generally less than $5 \times 10^{-9}$ Torr. There 


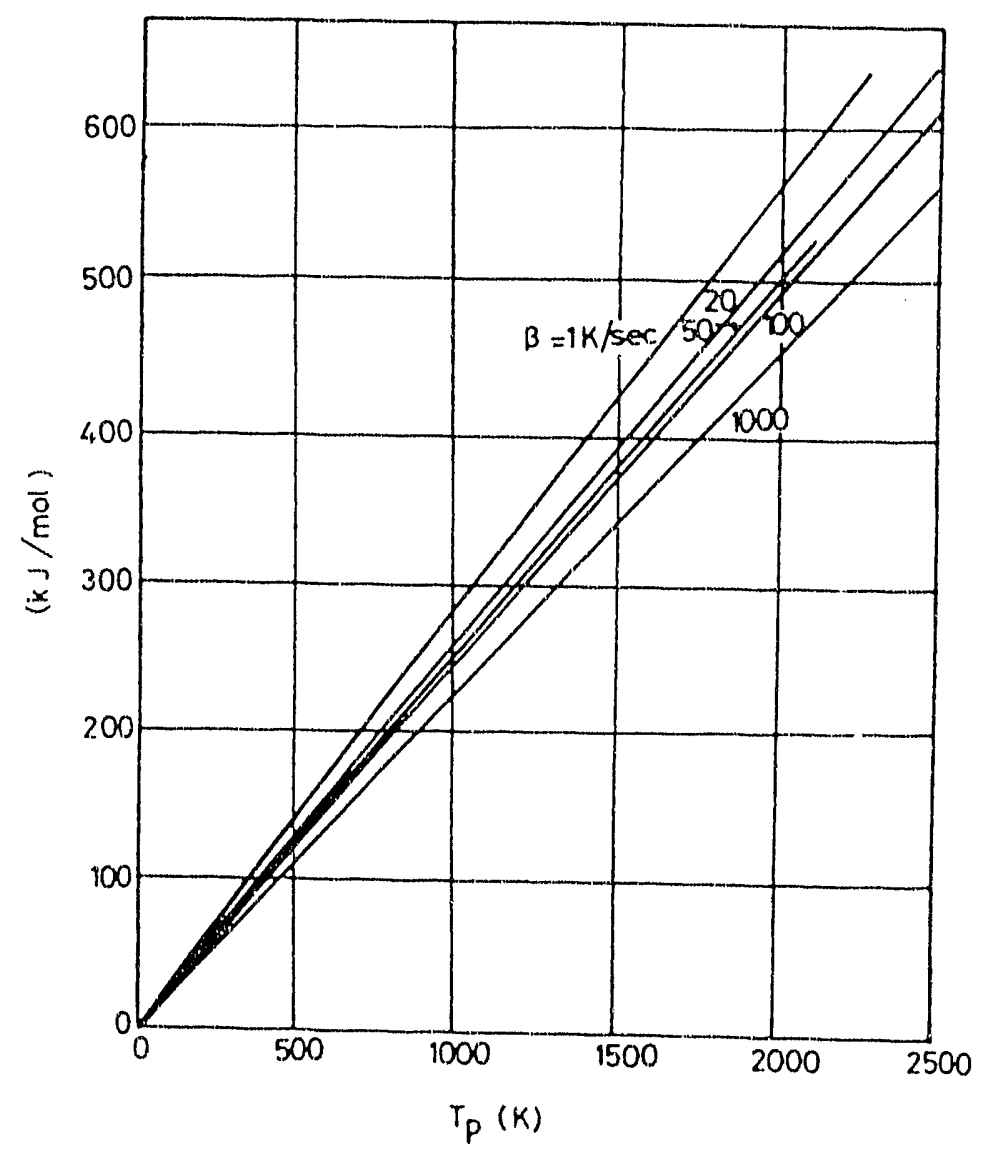

Figure 2.15: Redhead's plot of first order activation energies of desorption as a function of the temperature at the maximum rate of desorption. 


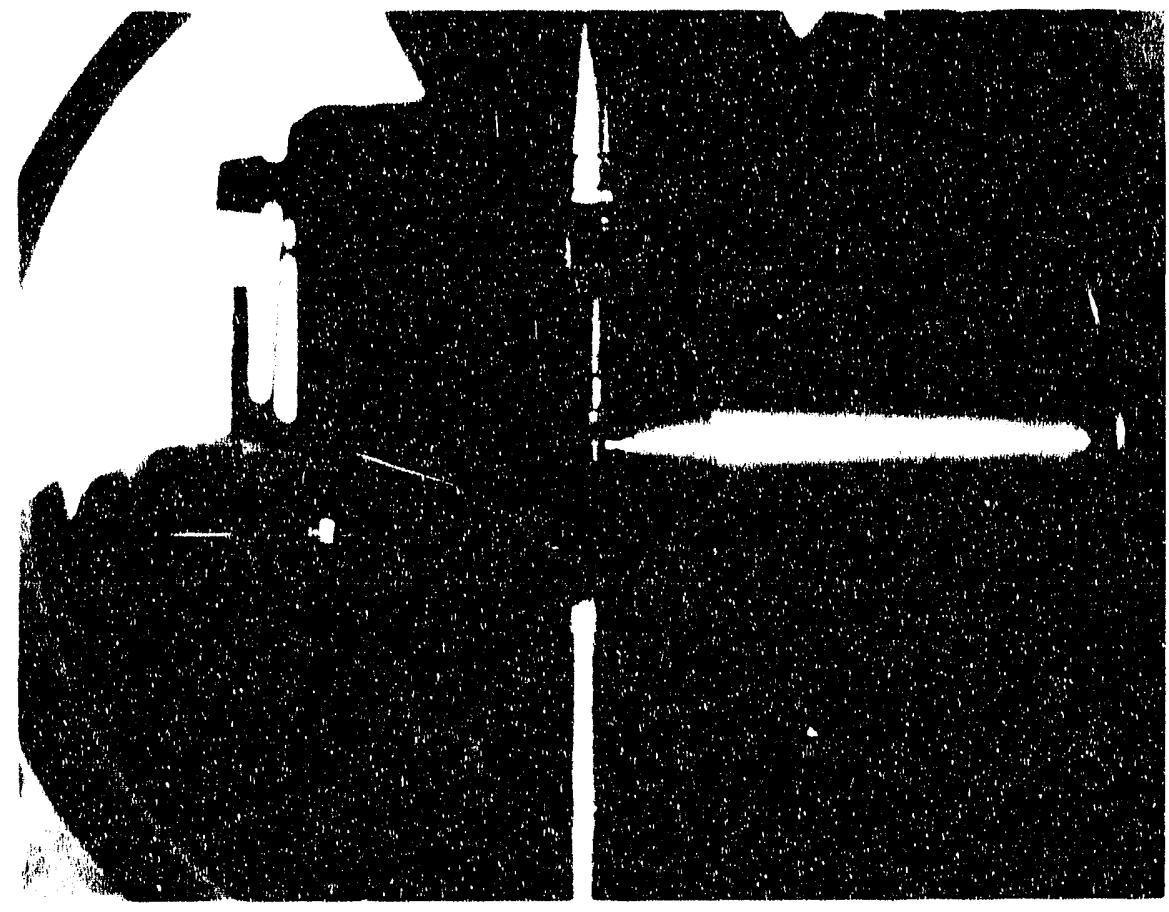

CBB 880-10492B

Figure 2.16: Schematic of thernal desorption experiment. 
was no noticeable rise in the chamber pressure during cobalt deposition. The crystal was heated at a constant rate of $\sim 35 \mathrm{~K} / \mathrm{sec}$. A linear ramp controller (7S3173) built in the building 62 electronic shop was used. This controller was actually linear in millivolts and not temperature. W 5\%Re/W $26 \% \operatorname{Re}$ thermocouples were sometimes used when performing desorption studies which required cooling the crystal below room temperature. Below approximately $230 \mathrm{~K}$, the voltage change with temperature is very small and inaccurate for $\mathrm{Pt} / \mathrm{Pt} 10 \% \mathrm{Rh}$ thermocouples.

\subsection{Sample preparation}

Cobalt, sulfur and carbon overlayers were deposited separately and in various combinations on different crystallographic surfaces of molybdenum single crystals. The preparation of these single crystals and the methods used to remove contaminants from their near surface region are described below.

\subsubsection{Preparation of single crystals}

From the unoriented single crystal rod, molybdenum single crystals exposing (100), (910), (911) and $(28,4,1)$ surfaces were cut and polished. Before orienting the rod, it was necessary to polish the surface until only one micron scratches were present. This was necessary in order to obtain sharp spots in the Laue diffraction pattern. The rod was placed in an appropriate holder to which it was physically and electrically connected using a conductive mixture of copper powder and duco cement. The rod in the holder was placed in a goniometer. Using the Laue machine in either buildings 2 or 62 , the rod was oriented so that the desired surface was parallel to the $\mathrm{x}$-ray beam. The crystals were oriented to 


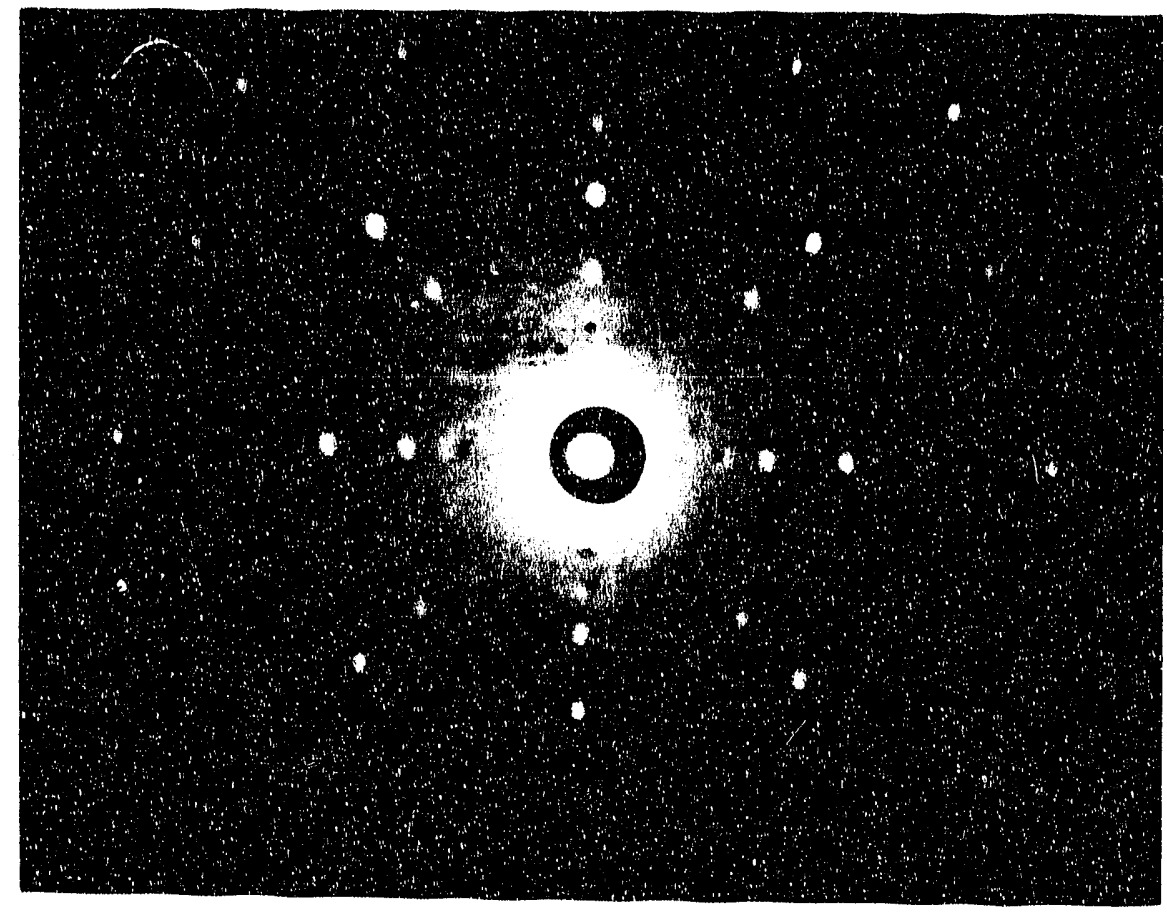

XBB 923-1802

Figure 2.17: Latke diffraction pattern of Mo(l(K)). 
within $1^{\circ}$ of the desired plane. A Laue diffraction pattern of the Mo(100) surface is shown in figure 2.17. This pattern shows a characteristic fourfold symmetry. To orient the other surfaces (all vicinal (100) surfaces), first the (100) plane was found and then the rod was turned by the necessary angle to bring the desired plane parallel to the $x$-ray beam. For example, turning a rod which has the $(100)$ surface parallel to the $x$-ray beam by $6.3^{\circ}$ (in either the $\mathrm{x}$ or $\mathrm{y}$ direction) towards the (110) plane (which is $45^{\circ}$ away from the (100) surface) will bring the (910) surface parallel to the $x$-ray source and therefore to the front of the goniometer. Following orientation of the rod, 1.5 millimeter thick slices were cut parallel to the front of the goniometer. Charles Covey in the building 62 student shop cut the slices using an electrical discharge machine. After cutting, the orientation of the crystals was rechecked. If the orientation was no longer within $1^{\bullet}$ of the desired face, the crystal was reoriented. The goniometer was then placed on the facing wheel in the metallography laboratory and the surface ground at the angle necessary to correct the orientation using twenty micron silicon carbide paper.

Once the orientation was correct, the crystals were mounted in Buehler transoptic powder. They were polished by sequentially using 15 and then 10 micron $\mathrm{SiC}$ paper, followed by 6 micron diamond paste on texmet and water slurries of 1,0.3 and finally 0.05 micron alumina on microcloth. The $\mathrm{SiC}$ paper and texmet were well lubricated with polishing oil. Surfaces were polished until few and if possible no scratches were visible. The crystal was removed after soaking overnight in acetone. It was remounted to polish the second side. Finally, the crystals were electropolished to remove the upper layers which might have been stressed from mechanical polishing and contaminated with polishing material. The electropolishing solution used contained twenty-five millimeters of concentrated sulfuric acid dissolved in one hundred and seventy-five millimeters of methanol. A strip of molybdenum foil was spotwelded to the edge of the crystal. This was placed in the center of a cylindrical stainless mesh which served as the anode. Successful 
results were obtained using a current of $\sim 2.2 \mathrm{~A}$ and a voltage of $\sim 30 \mathrm{~V}$. The electropolishing was performed for one minute at room temperature.

\subsubsection{Crystal cleaning procedures}

Carbon, sulfur and oxygen contaminants were seen by Auger spectroscopy on all new crystals. The major and most tenacious impurity was carbon, which could be removed by repeated heating in $5 \times 10^{-7}$ Torr of oxygen at $1600 \mathrm{~K}$. The oxygen was then pumped away and the crystal rapidly heated to $1900 \mathrm{~K}$ to desorb CO and to remove surface oxygen. The desorption of oxygen however, is not complete until $>2000 \mathrm{~K}$. When using $\mathrm{Pt} / \mathrm{Pt} 10 \% \mathrm{Rh}$ thermocouples, it was not possible to heat above $1900 \mathrm{~K}$ without melting the thermocouple. During periods when catalytic reactions were done, $\mathrm{Pt} / \mathrm{Pt} 10 \% \mathrm{Rh}$ thermocouples were used. During some thermal desorption experiments, W 5\%Re / W $26 \% \operatorname{Re}$ thermocouples were used. W 5\%Re / W $26 \% \operatorname{Re}$ thermocouples could not be used all the time since they become brittle in the high hydrogen pressures (1 atm) used in catalytic reactions. In the process of heating to $1900 \mathrm{~K}$, sulfur is desorbed. After ridding the surface of carbon, oxygen left on the surface was removed by dosing with $2 \times 10^{-8}$ Torr of ethylene followed by heating to $1900 \mathrm{~K}$. Generally to clean the surface of both carbon and oxygen, alternate treatments of oxygen and ethylene were required.

Cobalt could be desorbed from the crystal by heating above $1700 \mathrm{~K}$.

\subsection{High pressure reactions}

After characterization of the single crystals, they were enclosed in the high pressure cell described in section 2.2.2. Then the reactant gases (typically 2 - 3 Torr of thiophene 
and 780 Torr of hydrogen) were introduced into the high pressure cell and reaction loop. Thiophene was introduced first. Before initial use, thiophene was fractionally distilled in a nitrogen atmosphere over metallic sodium. The middle fraction of the distillate (boiling point $198 \mathrm{~K}$ ) was collected in an air tight vial containing molecular sieves with nominal pore diameter of $3 \AA$. These molecular sieves are suitable for dehydrating organic liquids. This fraction was subsequently freeze thawed to degas the thiophene and remove the nitrogen and air in the vial. The freeze thaw process was repeated until the residual gas pressure above the thiophene at $\sim 77 \mathrm{~K}$ was less than $10^{-3}$ Torr. Hydrogen was passed through a liquid nitrogen trap to remove less volatile contaminants (oxygen, water). Once hydrogen was intr duced in the reaction loop before thiophene. During the reaction, the 0.125 inch tantalum rods dissolved hydrogen and cracks were formed all through them. After the cell was pumped down and opened, the crystal was heated. As this was being done, hydrogen desorbed from the tantalum rods to the extent that the chamber pressure rose above $10^{-3}$ Torr. Apparently, when thiophene is introduced first, it adsorbs on the tantalum rods and prevents the permeation of hydrogen.

The reactant gases are circulated with a teflon pump (flow rate $\sim 50 \mathrm{cc} / \mathrm{min}$ ) for about five minutes. The crystal is then heated to the reaction temperature and maintained there using a temperature controller (LBL 7S2162). The reaction temperature was generally $613 \pm 3 \mathrm{~K}$, but it was varied to determine the activation energy of the reaction over different surfaces. Periodically, ( every fifteen minutes), samples of reactant gas mixture were injected into the gas chromatograph to monitor the extent of reaction. A fourty inch, 0.125 inch wide stainless column containing $19 \%$ picric acid on carbowax was capabie of separating the four carbon products produced in the reaction. The column was maintained at $303 \mathrm{~K}$ for four minutes to separate the hydrocarbon products, and then heated to $383 \mathrm{~K}$ at a rate of $32 \mathrm{~K}$ per minute. It was maintained at this temperature for an additional four minutes to cause the elution of thiophene. Typical retention times of the 
Table 2.2: Retention times of compounds

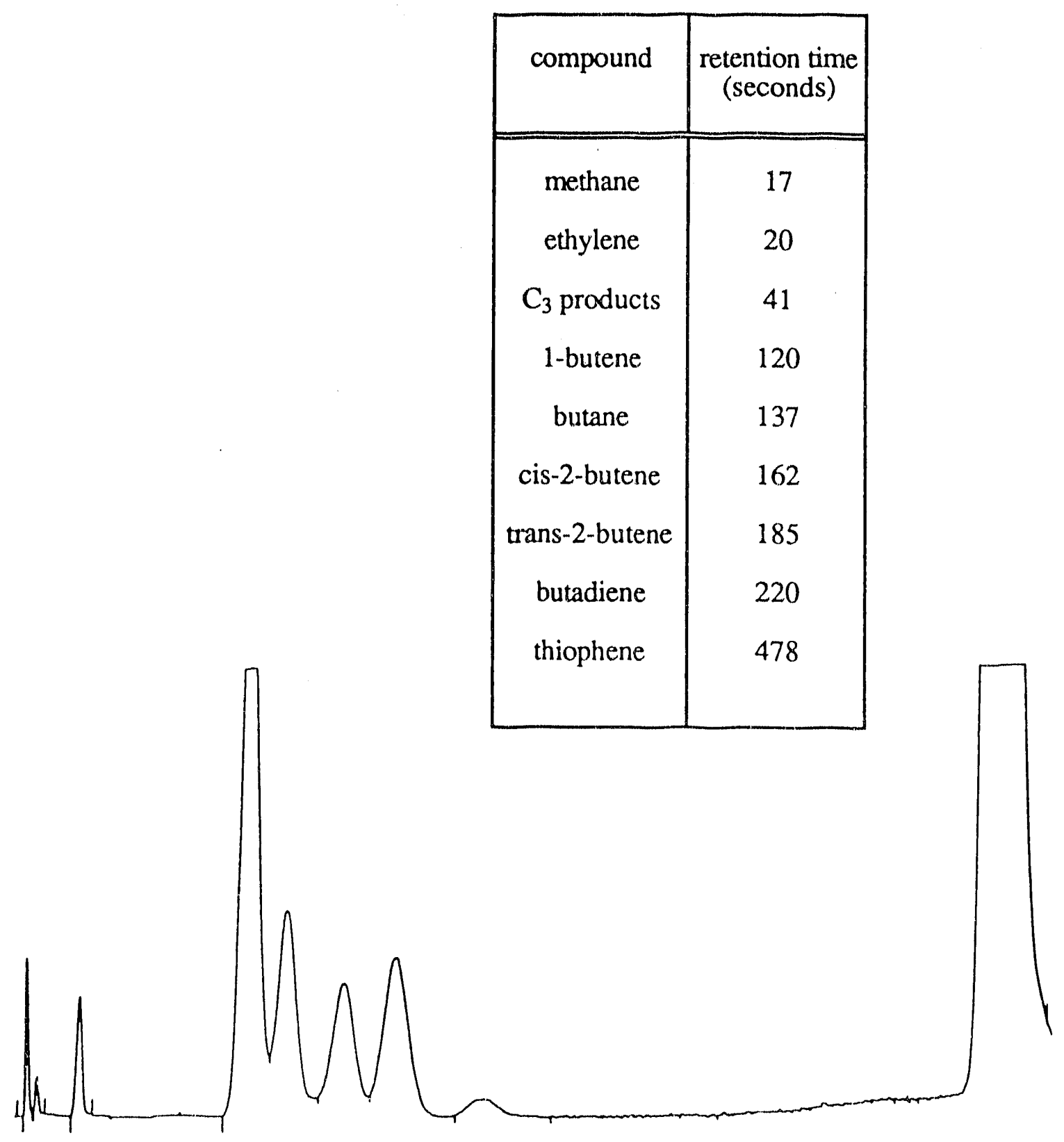

Figure 2.18: Gas chromatograph of compounds detected in thiophene HDS over Mo single crystal surfaces. 
hydrocarbon products are shown in table 2.2. Once separated, the products were detected using a calibrated flame ionization detector and the peaks areas plotted and integrated using a 3392A Hewlett Packard integrator. A gas chromatograph of the compounds detected is shown in figure 2.18 .

\section{References}

[1] H. Ibach in Electron Spectroscopy for Surface Analysis, Springer-Verlag Berlin, Heidelberg, New York (1977).

[2] A. Zangwill, Physics at Surfaces, Cambridge university press, Cambridge, New York, Melbourne, (1988).

[3] M. E. Bussell, Ph.D. thesis, University of California, Berkeley (1988).

[4] C. Wagner, J. Chem. Phys. 211819 (1953).

[5] G. Ertl and J. Küppers, Low Energy Electrons and Surface Chemistry, VCH (1985).

[6] P. W. Palmberg, G. E. Riach, R. E. Weber and N. C. MacDonalc, Handbook of Auger Spectroscopy, Physical Electronics Industries, Inc., Minnesota.

[7] C. Davisson and L. H. Germer, Phys. Rev. 30705 (1927).

[8] M. A. Van Hove, W. H. Weinberg and C. -M. Chan, Low-Energy Electron Diffraction, Springer-Verlag Berlin Heidelberg (1986).

[9] communication with Alan Lyon, Lawrence Berkeley Laboratory

[10] A. Abdo, R. F. Howe, and W. K. Hall, J. Phys. Chem., 82969 (1978).

[11] G. A. Somorjai, Chemistry in Two Dimensions: Surfaces, Cornell university press Ithaca, NY (1981).

12] D. A. King, Surf. Sci. 47384 (1975). 
[13] P. A. Redhead, Vacuum 12203 (1962). 


\section{Chapter 3 Structure of the Clean Vicinal Mo(100) Surfaces}

\subsection{Introduction}

In the previous chapter we described the analytical techniques used in this work to characterize single crystal surfaces. In this chapter, one of these techniques, low energy electron diffraction, is used to determine the structure of stepped and kinked molybdenum surfaces. Section 3.2 describes how information about the step height, step direction and terrace width can be determined from diffraction experiments. These methods are then applied in the following section to determine the structure of the clean vicinal surfaces.

Complex heterogeneous catalysts are composed not only of low Miller index surfaces, but also of high Miller index surfaces that may have high concentrations of atomic seps and kinks. In a structure sensitive reaction, the rate depends on the crystallographic orientation of the catalytic surface and consequently very different reaction rates can bc observed over surfaces witl. different Miller indices ${ }^{1,2}$. There are indeed instances where high Miller index surfaces have been shown to be more important for catalytic activity than their closed packed counterparr Therefore it is importarit to model catalys : using a variety of both low and high index surfaces. Up tn now, single crystal studies modelling the cobalt promoted molybdenum sulfide catalysıs have concentrated on closed packed faces and particularly the $M o(100)$ surface $e^{3,4}$. In this thesis, we have extended single crystal studies to include more highly corrugated surfaces. We have investigated vicinal (stepped $(910)$ and $(911)$ and kinked $(28,4,1)) \mathrm{Mo}(100)$ surfaces. 


\subsection{Diffraction From Stepped Surfaces}

As discussed in section 2.5.1, vicinal (100) surfaces can be prepared by cutting a single crystal rod inclined at a small angle away from the low Miller index (100) face. The resulting high Miller index surfaces are composed of low Miller index terraces separated by steps (figure 3.1(b)). However the macroscopic orientation of the surface does not uniquely define the terrace width and step height since a surface with monoatomic steps and terraces of a given width has the same macroscopic orientation as a surface with diatomic steps and terraces of doubled width. Indeed a surface can facet and still maintain the overall macroscopic orientation. Schematics of these possibilities are shown in figure 3.2. Therefore it is necessary to use diffraction studies to determine the step height, terrace width, and if facetting has occurred. Steps can themselves be composed of either low or high Miller index surfaces. Stepped surfaces in which the steps are high Miller index surfaces (the steps are composed of steps) are called kinked surfaces (figure 3.1(c)). Kink atoms have lower coordination than step atoms which in turn have lower coordination than terrace atoms.

The presence of steps and other defects can be detected by observation of the shape of diffraction beams $s^{6,7,8}$. These defects can be quantitatively characterized using a kinematic approximation (without including mulaple scattering events). This simplification allows the acquisition of quantitative information about the size and defect structure without significant computational effort. Since multiple scattering is not included, peak intensities are not correctly reproduced. Hence, spot profile analysis does not provide information on atornic positions but only on deviations from periodicity. The necessary experimental data are obtained by measuring the spot intensity on the screen as a function of wave vector transfer $\left(I\left(\underline{k}_{\|}\right)\right)$. Of surface defects, steps are the most widely studied using electron diffraction. This is probably because the presence of steps modify the diffraction pattem in 
(a)

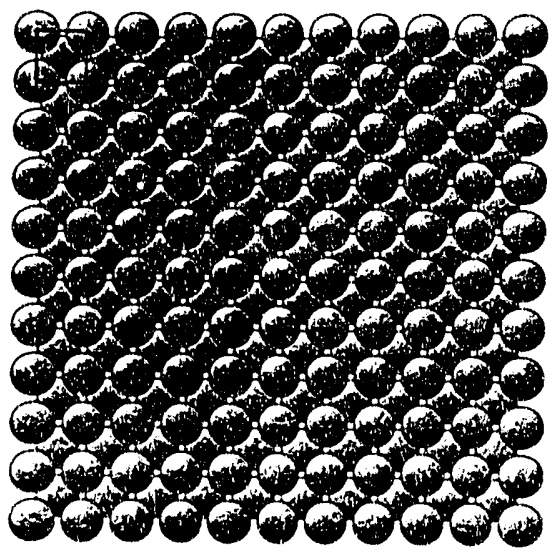

(b)

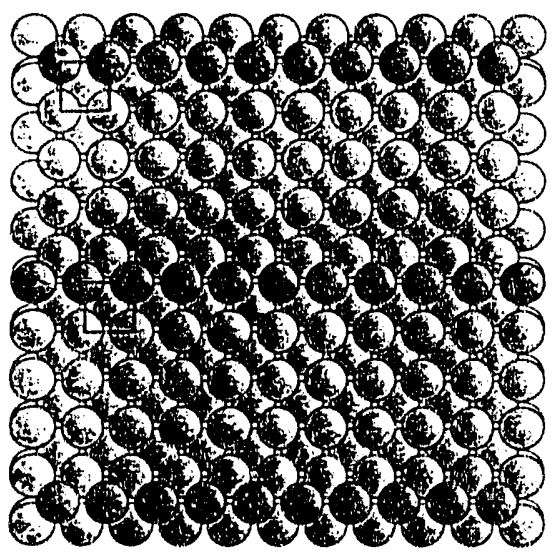

(c)

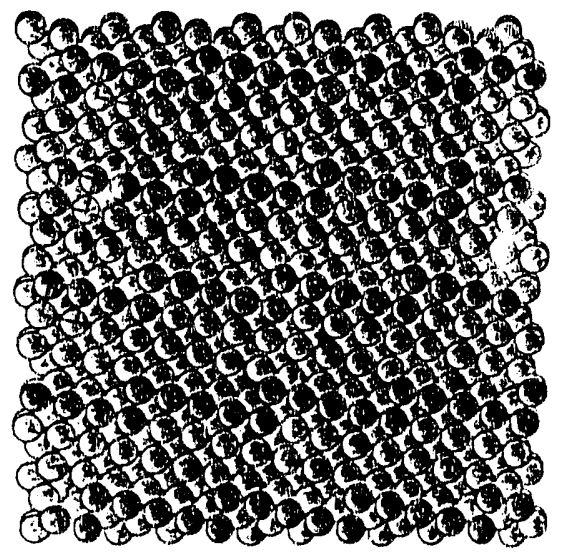

Figure 3.1: Top views of (a) a low Miller index (100) surface, (b) a corresponding stepped (910) and (c) a corresponding kinked $(28,4,1)$ surface. The darker circles represent the step and kink atorns. 


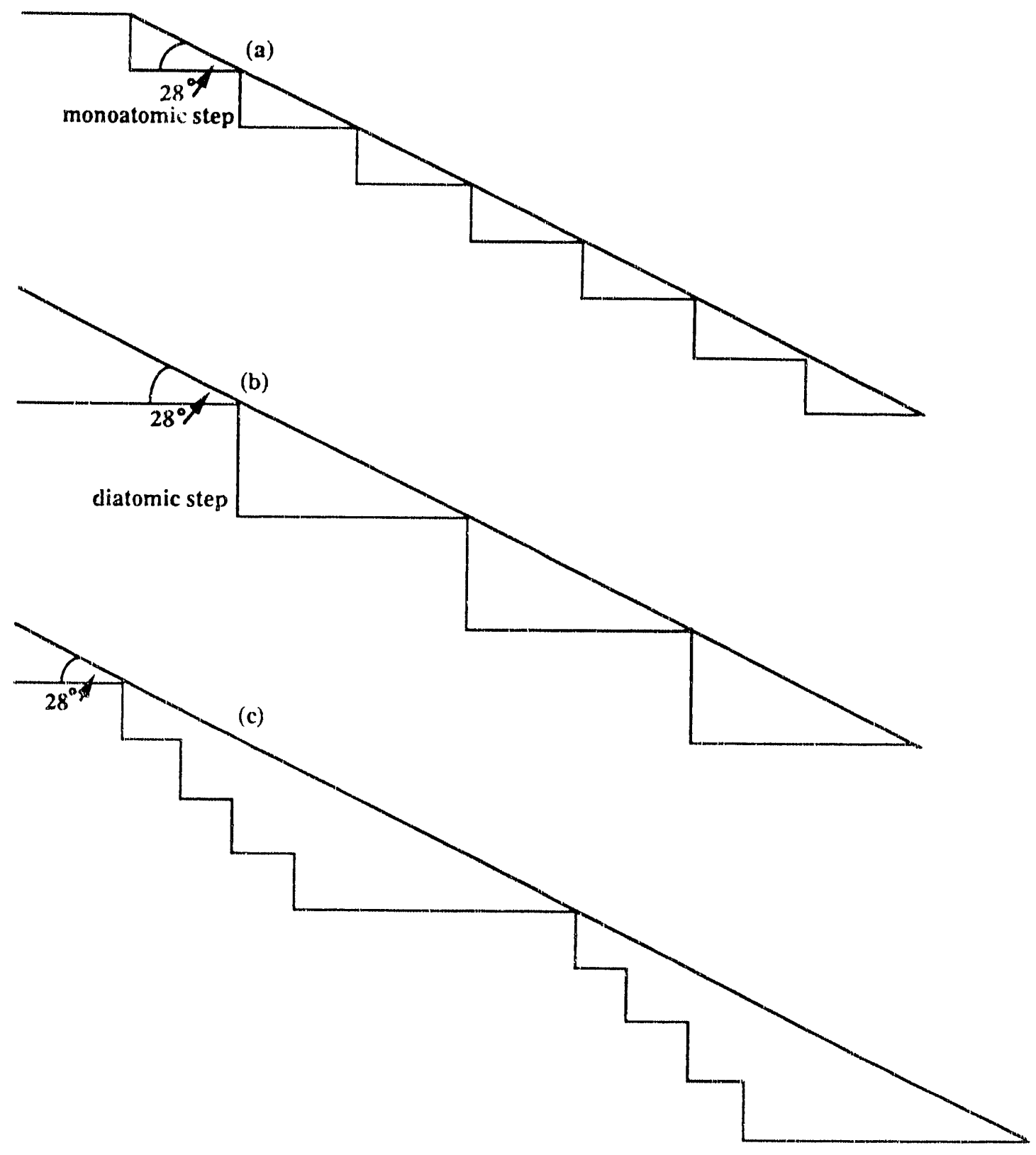

Figure 3.2: Schematics showing that a high Miller index surface with a given macroscopic orientation can have (a) monoatomic steps, (b) diatomic steps or even (c) facets. 
a clearly predictable manner. Diffraction experiments allow the determination of the terrace width, step height and direction, enabling a description of the structure of stepped surfaces.

Regular atomic steps on single crystal surfaces cause LEED beams to have spot profiles that oscillate between doublets and singlets as the primary energy is varied. The voltages at which singlets are seen correspond to constructive interference of electrons scattered from atoms located on adjacent terraces. Doublets are seen at energies where electrons from atoms on neighboring terraces scatter out of phase. Figure 3.3 shows a schematic of constructive and destructive interference at a surface with monoatomic steps. Each non-equivalent set of diffraction beams has characteristic voltages at which the beams appear as doublets.

Spot splitting can also be seen using the Ewald construction shown in figure $3.4^{9}$. The macroscopic surface orientation depends on the periodicity of the steps. In the Ewald construction, reciprocal lattice rods are drawn perpendicular to the macroscopic surface. A large unit cell is associated with the step periodicity, hence these reciprocal rods are closely spaced. The corresponding surface rods due to the terrace periodicity are also drawn. As discussed in section 2.3.2, in the Ewald construction, a sphere is drawn with a radius corresponding to the incident momentum wave vector. The intersections of the sphere with reciprocal lattice rods determine the angles at which diffracted beams appear. The Ewald sphere intercepts many rods of the macroscopic surface, giving rise to many closely spaced, but too weak to be visible, diffraction bearns. However when the Ewald sphere intercepts macroscopic surface rods that lie close to terrace rods, bright diffraction beams are seen in these directions. A given macroscopic rod becomes visible when it's intersection with the Ewald sphere is close to a terrace rod. As the energy is changed, the intersection between the Ewald sphere and the macroscopic surface rod becomes closer to the terrace rod resuiting in an increased intensity due to that macroscopic rod. This intensity reaches a maximum when the location of the macroscopic rod, terace rod and 


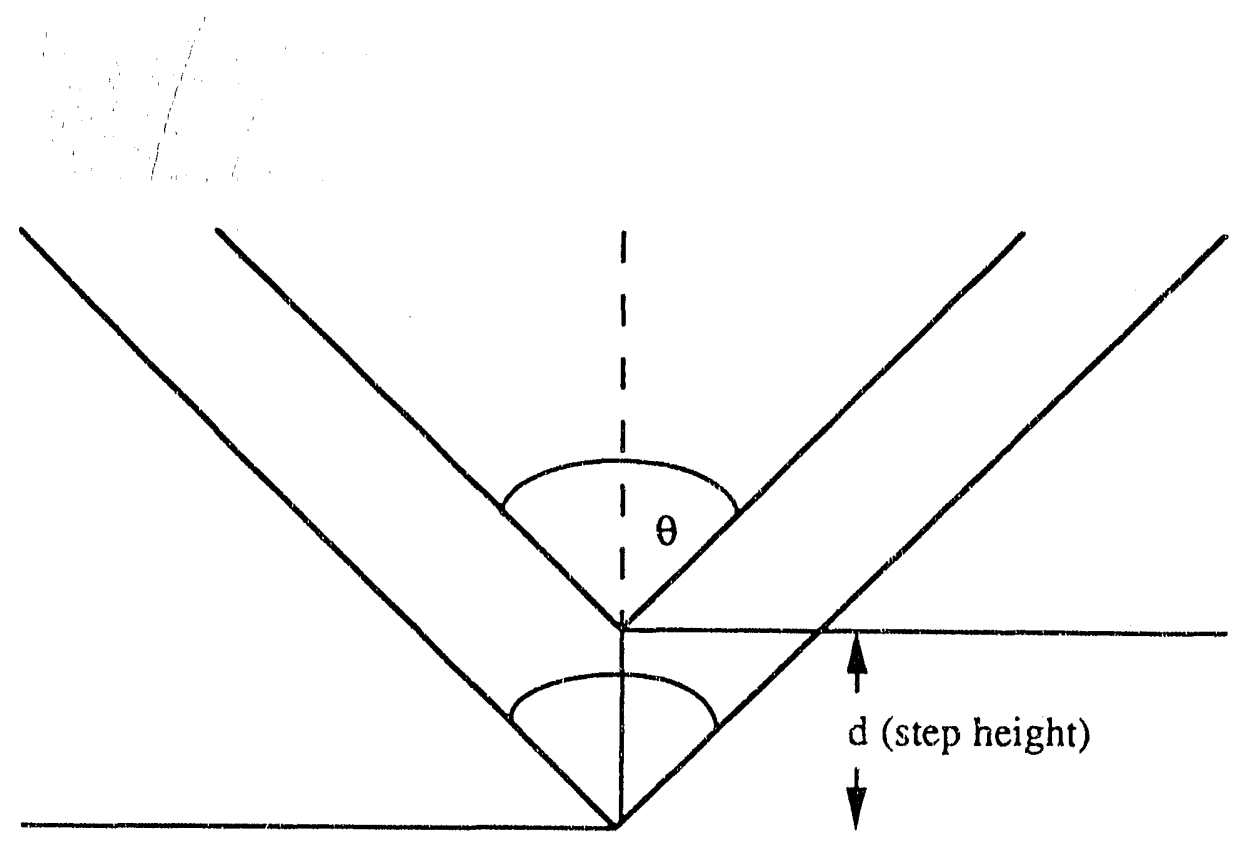

$2 \mathrm{~d} \sin \theta=\mathrm{s} \lambda$

when $s=$ integers, there is constructive interference

(00) beam appears as a singlet

when $\mathrm{s}=$ half integers, there is destructive interference

$(00)$ beam appears as a doublet

for electrons: $\lambda(\AA)=(150 / E(e V))^{1 / 2} \quad(p 46)$

at normal incidence $\theta=90^{\circ}$

Therefore

$E=\frac{150 s^{2}}{4 d^{2}} \quad$ (He) $z$ zler's equation for the $(00)$ beam $)$

(p 72)

Figure 3.3: Constructive and destructive inteference àt à siêpped sur face. 

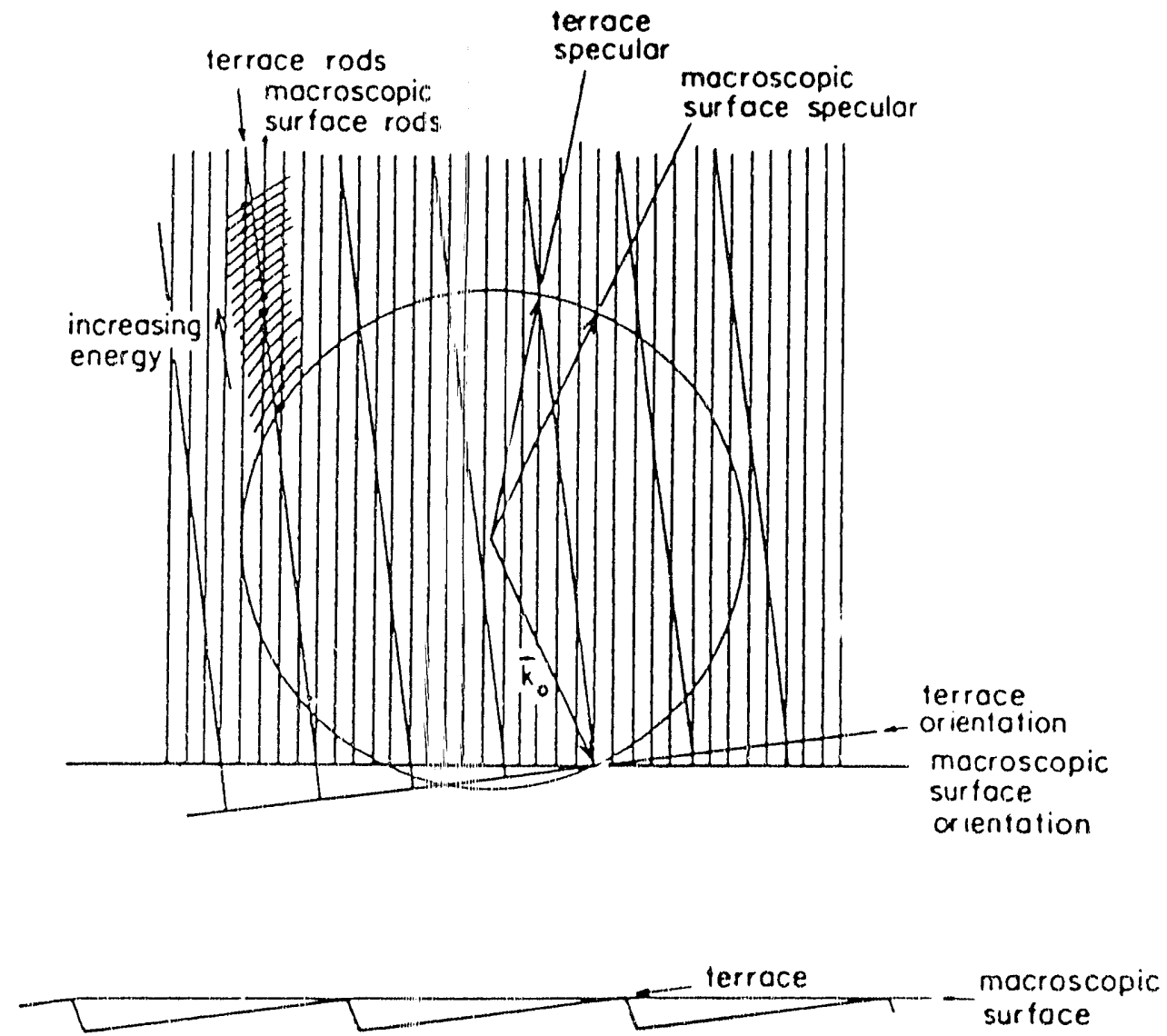

Figure 3.4: Ewald construction for stepped surfaces. 
Ewald sphere exactly coincide in $\mathrm{k}$ space. Since intense beams are only seen in the directions determined by the terrace rods, the general diffraction pattern (minus spot splitting) of vicinal surfaces is similar to those of the corresponding flat surfaces. Since the terrace rods are not thin due to the small terrace width and the macroscopic rods are closely spaced, generally more than one macroscopic rod will lie close to a terrace rod and hence doublets (or in general multiplets) will be seen. At one particular energy the components of a doublet will have equal intensity. At lower energies, one member of the doublet will have higher intensity and at higher energies, the other member will be brighter. The effect of increasing the energy and hence the radius of the Ewald sphere is also shown. By changing the energy, the Ewald sphere will intercept different macroscopic surface rods that are close to a terrace rod, making the diffraction beams due to these rods in turn visible. Hence, at one energy a given beam will appear as a singlet and another as a doublet, while at a different energy the reverse might be seen.

The energies at which singlets and equally intensive doublets are seen have been derived by Henzler ${ }^{6-8}$ using a kinematic approximation. The different consecutive energies at which singlets and equally bright doublets are seen are determined using consecutive whole and half integer values for $s$ in the Henzler equation. From the energy dependence of the spot splitting, the step height can be determined. Henzler's general equation is shown below for normal incidence.

$$
\begin{aligned}
V_{h, k}= & \frac{150}{4 d^{2}}\left(s-h x-k y+\frac{d^{2}}{4 \pi^{2}} \frac{\left(h a^{*}+k b^{*}\right)^{2}}{s-h x-k y}\right)^{2} \\
a^{*}, b^{*}= & \text { surface reciprocal lattice vectors } \\
x, y= & \text { defined such that } 0 \leq x, y \leq 1 \text { give the horizontal shift } \\
& \text { between the terrace unit cell of the upper and lower terrace } \\
h, k= & \text { define the particular ciffraction beam }
\end{aligned}
$$




$$
\begin{aligned}
& d=\text { step height } \\
& s=\text { whole or half integer, } s \geq \frac{\left|h a^{*}+k b^{*}\right| d}{2 \pi}+h x+k y
\end{aligned}
$$

The voltages at which a given beam appears as a singlet or doublet depends $n$ the step height (d), terrace lattice parameter (a) and the horizontal shift between thr wit cell in the upper and lower terraces ( $\mathrm{g}$ ) (figure 3.1). These voltages are independent of the orientation or width of the terrace.

The direction of spot splitting indicates the direction of the step edge periodicity. Since the diffraction pattern is a map of the reciprocal lattice of the surface, the steps run perpendicular to the observed direction of spot splitting . The relative separation between the spots in a doublet depend on the average terrace width. This relationship is shown below.

$$
\begin{aligned}
\frac{\Delta \mathrm{K}_{01}}{\mathrm{~K}_{01}} & =\frac{\mathrm{a}}{\mathrm{w}} \\
\text { where } & \\
\Delta \mathrm{K}_{01} & =\text { distance between split spots in (01) direction } \\
\mathrm{K}_{01} & =\text { distance between integer spot positions in (01) direction } \\
\mathrm{a} & =\text { terrace lattice constant } \\
\mathrm{w} & =\text { average terrace width }
\end{aligned}
$$

If the surface has terraces of randorn width but steps of constant height, diffuse rather than split spots are observed. For these surfaces, the different doublets due to terraces of different widths add up to produce an overall diffuse spot. If there is preferential orientation of the steps, then the diffuse spot will appear elongated in the direction perpendicular to the preferred step direction. In these cases profile analysis of the spot shape (deviations from circular symmetry) provide information on the presence of 
surface defects.

In summary, the step height is determined by the energies at which spot splitting occurs, the step orientation by the direction of spot splitting on the diffraction pattern and the terrace width by the relative separation between spots in a doublet.

\subsection{Characterization of Stepped and Kinked Mo(100) Surfaces}

The diffraction patterns of the clean vicinal surfaces are shown in figure 3.5. The step height was determined for the clean vicinal surfaces using Henzler's equation. For monoatomic steps on vicinal (100) surfaces of body centered cubic materials, the terace unit cell on consecutive terraces is shifted by one half of a lattice constant in both the $\mathrm{x}$ and $y$ direction. Therefore in these cases, $x=y=0.5$. For diatomic steps, there is no shift between the terrace unit cell on consecutive terraces. Therefore for diatomic steps on vicinal $(100)$ body centered cubic materials $x=y=0$. The surface reciprocal lattice vectors of the (100) surface of molybdenum are defined as $a^{*}=b^{*}=2 \pi / a=2 \pi / 3.15 \sim 2$. In the case of monoatomic steps, the step height is $1.57 \AA$, and for diatomic steps, it is $3.15 \AA$. The step height is a multiple of the interplanar distance of the bulk layers that are perpendicular to the terrace. This relationship holds even when there is a relaxation of the topmost surface layers. In these cases, identical relaxations of all terrace layers ensure that he step height remains equal to the appropriate bulk interplanar distance. By substituting the required numbers into Henzler's equation, the energies at which the (01) and symmetrically equivalent beams appear as singlets and doublets for monoatomic and diatomic steps have been calculared. These voltages are given in table 3.1. It is clear from this table that the oscillation between singlets and doublets occur with greater frequency in 

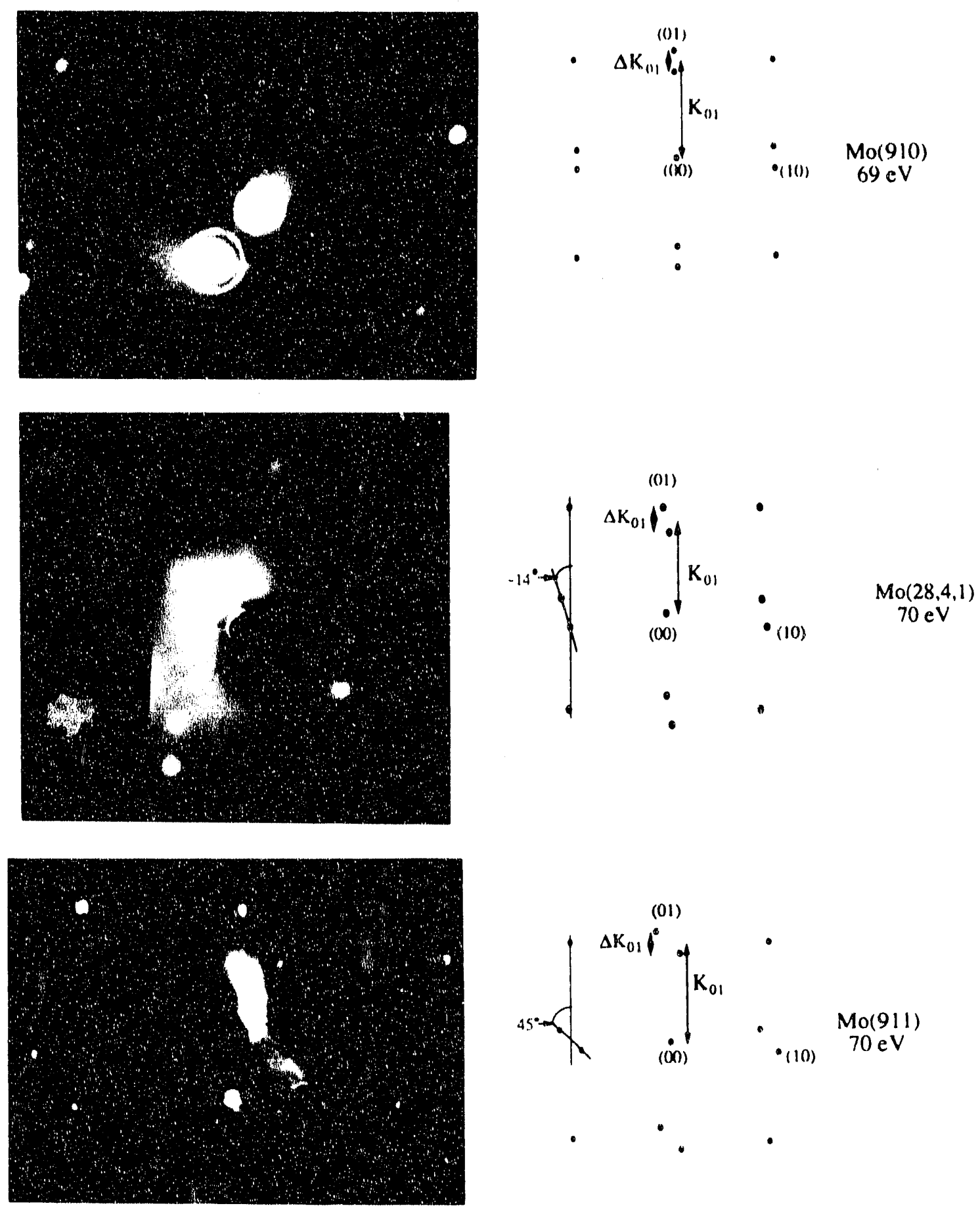

XBB $910-8136 \mathrm{~A}$

Figure 3.5: Diffraction pallerns of the clean vicinal surfaces. Schematic representations of the diffaction patterns are also shown. On the schematics, the (()()), (()1) and (10) beams, $\Delta \mathrm{K}_{(01}$ and $\mathrm{K}_{01}$ are labelled. 
Table 3.1: Calculated energies at which singlets and doublets are seen for monoatomic and diatomic steps on vicinal $\mathrm{Mo}(100)$ surfaces for the symmetrically equivalent $(01),(0 \overline{1}),(10)$ and $(\overline{1} 0)$ beams.

\begin{tabular}{|c|c|c|c|c|}
\hline & \multicolumn{2}{|c|}{ monoatomic steps } & \multicolumn{2}{|c|}{ diatomic steps } \\
\hline$s$ & singlets & doublets & singlets & doublets \\
\hline 1.5 & & 24 & & 18 \\
\hline 2 & 42 & & 24 & \\
\hline 2.5 & & 70 & & 32 \\
\hline 3 & 103 & & 42 & \\
\hline 3.5 & & 144 & & 54 \\
\hline 4 & 194 & & 69 & \\
\hline 4.5 & & 251 & & 85 \\
\hline 5 & & & 105 & \\
\hline 5.5 & & & & 123 \\
\hline 6 & & & 146 & \\
\hline 6.5 & & & & 168 \\
\hline 7 & & & 193 & \\
\hline 7.5 & & & & 221 \\
\hline 8 & & & 251 & \\
\hline 8.5 & & & & 282 \\
\hline
\end{tabular}


the case of diatomic steps. The energies at which singlets and doublets for the (01) and symmetrically equivalent beams are seen for the (910), (911) and $(28,4,1)$ surfaces are given in table 3.2. By comparison with the frequency of oscillation between singlets and doublets, it is apparent that there is better agreement of the experimental data if monoatomic steps are assumed. Since monoatomic steps are present on the three clean vicinal surfaces studied, spot splitting is observed at similar energies for all these surfaces.

The deviations between the experimental and calculated energies arise from several sources. The energies where sharp singlets and equally bright doublets were seen was determined by observation of the diffraction pattern. A more quantitative determination would involve measuring the beam intensities and their full width at half maximum. Also in the calculations, the electron beam was assumed to be normal to the macroscopic surface. This orientation was only approximately realized (within $\sim 10^{\circ}$ ). Finally, Henzler's equation used to determine these energies neglects any relaxation of the step atoms. A downward displacement of the atoms on the step edges of the upper terrace and or an upward displacement of the instep atoms of the lower terrace might change the energies of maximum intensity of singlets or equally bright doublets.

The diffraction patterns of the vicinal surfaces are shown in figure 3.5. From these patterns and the energies of spot splitting, the step height, step orientation and terrace width was determined. For all of the surfaces these values agree with the macroscopic orientation defined by the cutting angle and determined by LAUE diffraction (section 2.4.1). We have determined that monoatomic steps are present on these surfaces. Therefore we can draw the co responding real space models shown in figure 3.6 using the Surface Architect (SARCH) program of Van Hove ${ }^{10}$. For the clean (910) surface, spots are split in the $(01)^{11}$ direction and therefore the steps run in the [001] direction of the crystal. From the diffraction pattern, it was determined that $\Delta \mathrm{K}_{01} / \mathrm{K}_{01}$ is approximately $1 / 4.7$ for the (910) surface. This result means that there are four and a half rows of molybdenum atom on an 
Table 3.2: Energies at which singlets and doublets are observed for the (01) beam for vicinal Mo(100) surfaces.

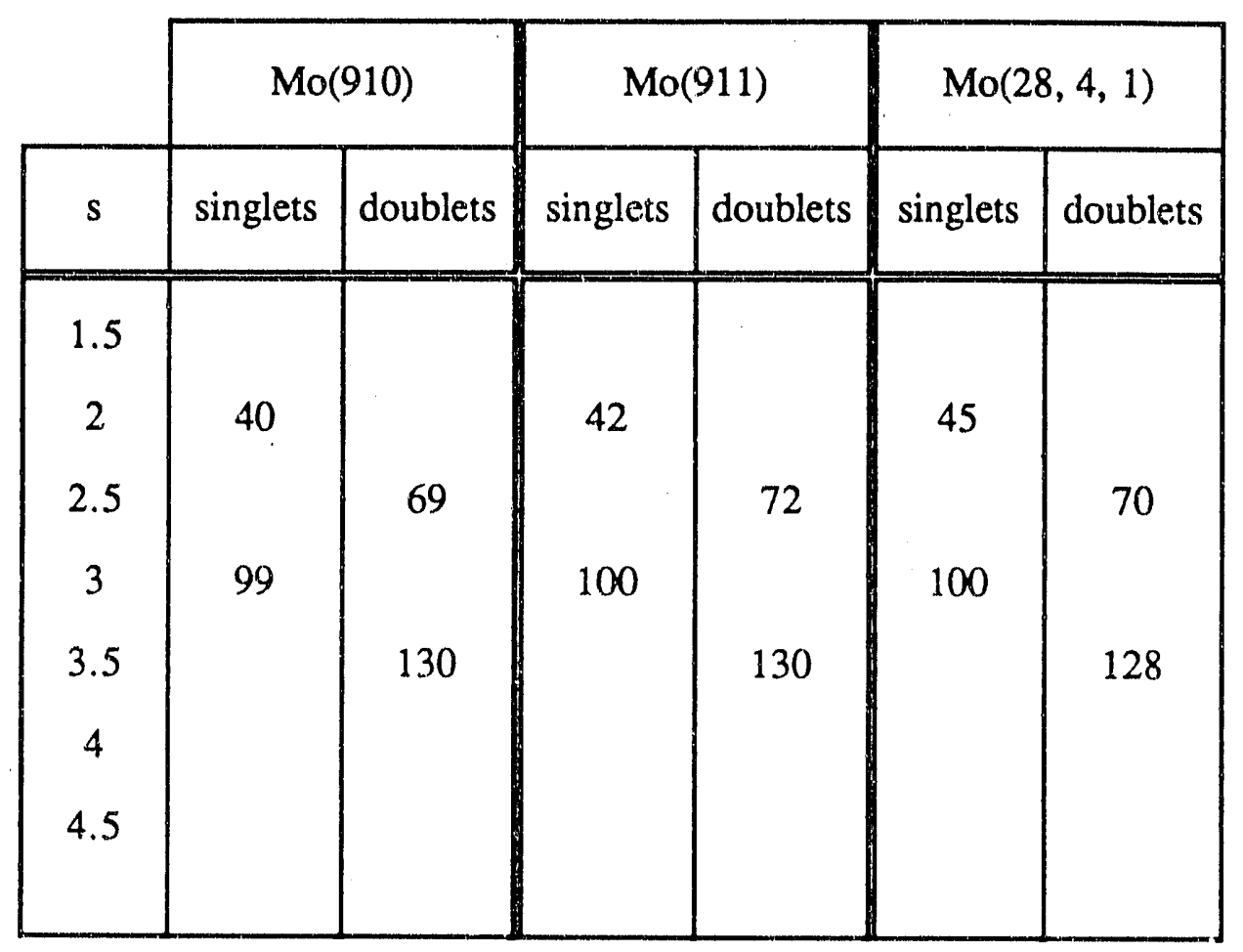

Table 3.3: Calculated number of terrace atoms on vicinal $\mathrm{Mo}(100)$ surfaces.

\begin{tabular}{|c|c|c|c|}
\hline Surface & $\Delta \mathrm{K}_{10} / \mathrm{K}_{10}$ & $\begin{array}{c}\text { exp't. determined \# } \\
\text { of terrace atoms }\end{array}$ & $\begin{array}{c}\text { ideal \# of terrace } \\
\text { atoms }\end{array}$ \\
\hline \hline $\operatorname{Mo}(910)$ & $0.5 / 2.3$ & $\sim 4.6$ & 4.5 \\
$\operatorname{Mo}(911)$ & $0.6 / 2.4$ & $\sim 4$ & alternating 4 and 5 \\
$\operatorname{Mo}(28,4,1)$ & $0.6 / 2.2$ & $\sim 3.6$ & 3.5 \\
\hline
\end{tabular}




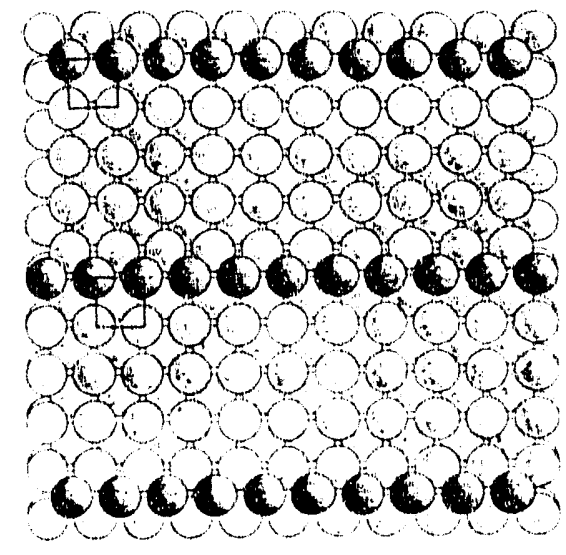

$+\left.[001]\right|_{[010]}[190]$

(a)
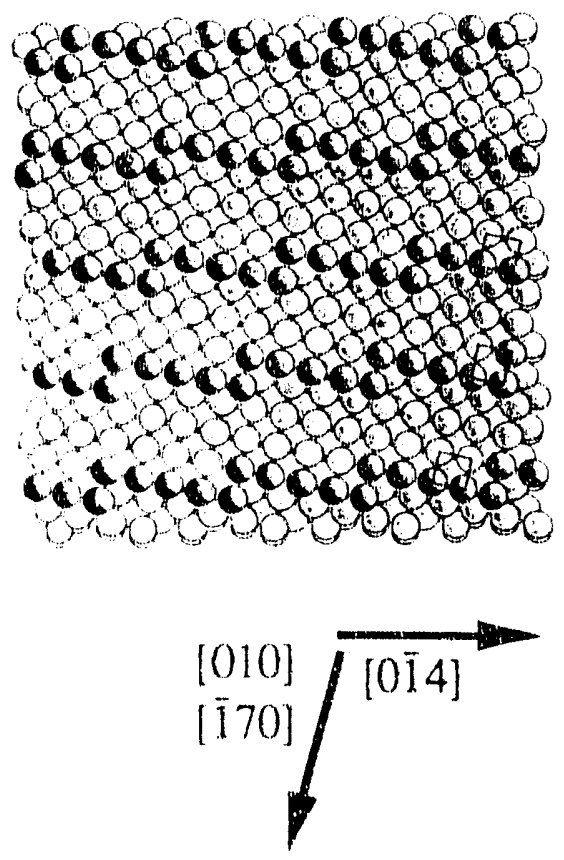

(b)
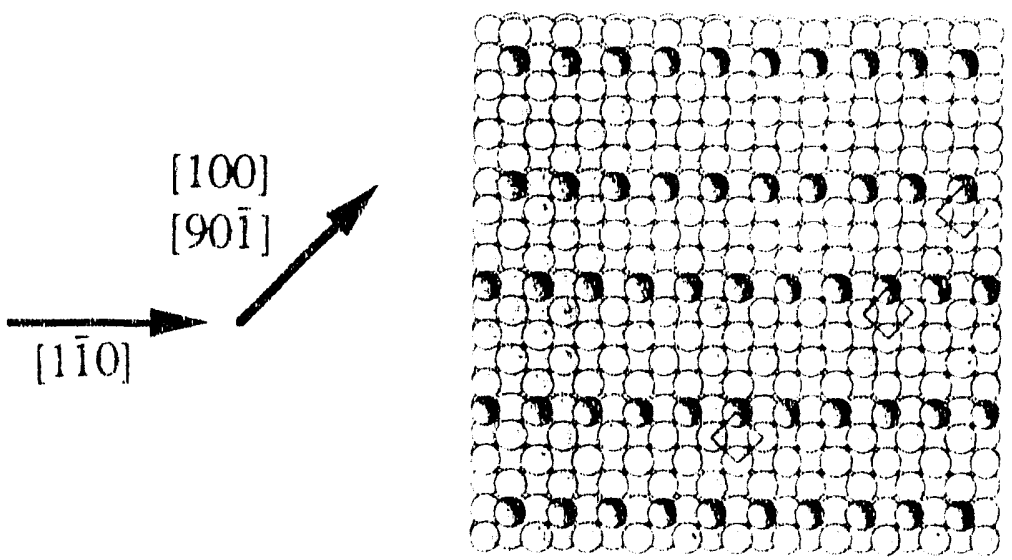

(c)

Figure 3.6: Diagrammatic representations of real-space (a) $\operatorname{Mo}(910)$, (b) $\operatorname{Mo}(28,4,1)$ and (c) Mo(911) surfaces. The upward arrow in (a) indicates both the [010] direction in the plane of the terrace and the [190)] direction which is parallel to the macroscopic surface. The downward arrow in (b) indicates both the [010] direction in the plane of the terrace and the [170] direction which is parallel to the macroscopic surface. The diagonal arrow in (c) indicates both the $[1(0)]$ direction in the plane of the verrace and the $[901]\}$ direction parallel to the macroscopic surface. 
average terrace. There is additionally a shift of half an atomic distance between neighboring terraces (figure $3.6(\mathrm{a})$ ). In the case of the kinked $(28,4,1)$ surface, the spots are split along the direction that is approximately $14^{\circ}$ away from the $(01)$ direction. For the $(28,4,1)$ surface, the size of spot splitting, $\Delta \mathrm{K}_{01} / \mathrm{K}_{01}$ is approximately $1 / 3.6$. This indicates that the average terrace is composed of three and a half rows of molybdenum atoms with a shift of half an atomic distance between neighboring terraces. Consecutive terraces are separated by monoatomic steps running along the [0 14$]$ direction (figure 3.6(b)). For the (911) surface, the spots are split aiong a direction that is $45^{\circ}$ away from the $(01)$ dir action. The size of spot splitting, $\Delta \mathrm{K}_{01} / \mathrm{K}_{01}$ is approximately $1 / 4$. This indicates that the average terrace is composed of about four molybdenum atom rows. Consecutive terraces are separated by monoatomic steps running along the [110] direction. From figure 3.5(c), it can be seen that alternating terraces actually have widiths of four and five molybdenurn atoms. There is a shift of half an atomic distance between the square unit cell on neighboririg terraces. The experimentally determined and calculated terrace widths of the vicinal surfaces used are shown in the table 3.3. Since surface crystallography (pp 43,50 ) has not been performed on these surfaces, the relaxation of atoms in the surface region and especially of atoms at the step edges has not been determined.

\subsection{Summary}

In this chapter, the step height, orientation and terrace width has been characterized for three vicinal $\mathrm{Mo}(100)$ surfaces. The $(910)$ and $(28,4,1)$ surfaces have sieps of similar orientation (figure $3.6(\mathrm{a})$, (b)). The difference between the step orientation on these two surfaces is that on the $(28,4,1)$ surface, steps rumning along the [iol] direction are interrupted every four atoms by monoatomic kinks in the [010] direction of the crystal. On 
the other hand, on the (911) surface, the steps are in a very different crystal direction. The step direction is $45^{\circ}$ away from the step direction on the (910) surface. Since these vicinal surfaces all have terraces of $(100)$ orientation, by comparison with the flat $(100)$ surface, we can investigate the effects of steps on chemisorption and reactivity (chapters $4-6$ ). Addicionally, we will take advantage of the different step directions to determine the effect of step orientation on the various adsorbate superstructucs formed on the terraces (chapter 5).

\section{References}

[1] M. E. Bussell, A. J. Gellman and G. A. Somorjai, J. Catal. 110423 (1988).

[2] M. Assher and G. A. Somorjai, Surf. Sci. 143 L.389 (1984).

[3] M. E. Bussell and G. A. Somorjai, J. Catal. 10693 (1987)

[4] M. E. Bussell, A. J. Gellman and G. A. Somorjai, Catal. Lett. 1195 (1988).

[5] G. A. Somorjai, Chemistry in Two Dimensions: Surfaces, Cornell University Press, Ithaca, NY (1980).

[6] M. Henzler, Surf. Sci. 2212 (1970).

[7] M. Henzler, Appl. Phys. 911 (1976).

[8] M. Henzler, Surf. Sci. 19159 (1970).

19] M. A. Van Hove, W. H. Weinberg and C. -M. Chan, Low-Energy Electron Diffraction, Springer-Verlag, Berlin Heidelberg (1986)

[10] M. A. Van Hove, Surface Architect Prograrn (1990).

[11] The (01) reciprocal space direction given is related to the terrace periodicity, ie. this vector is identical to (01) vector on the flat $(100)$ surface. 


\section{Chapter 4 \\ Growth of Cobalt Overlayers on Clean, Sulfur and Carbon Covered Molybdenum Surfaces}

\subsection{Introduction}

The goal of this chapter is to examine the growth mode of cobalt overlayers on flat and vicinal $\mathrm{Mo}(100)$ surfaces and the influence of adsorbates on the growth mode. The preparation and characterization of the clean vicinal surfaces were discussed in sections

2.4.1 and 3.3. Auger electron spectroscopy and thermal desorption spectrometry are the primary techniques applied in this chapter. In section 4.2 , some thermodynamic and kinetic factors which play roles in determining the growth mode of overlayers are discussed. Section 4.3 describes the use of Auger spectroscopy to determine the growth mode and coverages of overlayers and section 4.4 describes the acquisition of binding energies from thermal desorption experiments. Auger spectroscopy along with carbon monoxide chemisorption are then employed in the following two sections to characterize the growth of cobalt overlayers on clean and adsorbate covered molybdenum surfaces. Finally, sections 4.1 and 4.8 compare thermal desorption spectra of cobalt overlayers from clean and adsorbate covered surfaces flat (4.7) and vicinal (4.8) Mo(100) surfaces. The results are summarized at the end of the chapter. 


\subsection{Thermodynamic and Kinetic Influences on Overlayer Morphology}

There are three common growth modes named after the investigators who first described them: Frank-Van der Merwe growth, Stranski-Krastanov growth and Volmer-Weber growth ${ }^{1,2}$. If the surface free energy of the substrate material is greater than the sum of the interfacial and surface free energy of the overlayer material $\left(\sigma_{\mathrm{s}}>\sigma_{\mathrm{o}}+\sigma_{\mathrm{i}}\right)$, Frank-Van der Merwe growth can be expected. It is thermodynamically favorable for the lower surface free energy overlayer material to completely wet and hence cover the substrate. Ideally, in this growrt/ mode, each layer is fully completed before the next layer is begun. The expected plots are shown schematically in figure 4.1 (a).

There are other cases when it is thermodynamically favorable for the overlayer to nucleate into three dimensional islands upon contact with the substrate. Three dimensional island generally grow when the sum of the interfacial energy and the surface free energy of the overlayer is greater than the surface free energy of the substrate $\left(\sigma_{s}<\sigma_{0}+\sigma_{i}\right)$. This growth mode is referred to as Volmer-Weber growth. In Volmer-Weber growth, islands several layers thick and exposed substrate patches can be simultaneously present on the surface (figure 4.1 (c)). Stranski-Krastariov growth is a compromise between these two extremes (figure 4.1(b)). This growth mode is expected when $\sigma_{\mathrm{s}} \sim \sigma_{\mathrm{o}}+\sigma_{i}$. In this growth mode, the first few monolayars grow in a layer-by-layer fashion. Additional monolayers grow in a Volmer-Weber fashion. Although calculations of the surface free energies of selected metais at $0 \mathrm{~K}$ are reported ${ }^{3}$, and some experimental data is available, interfacial energies are commonly not reported. This limits the use of these criteria in determining which growth mode should be expected.

The interfacial energy is comprised of a chemical and structural component. The chemical component is determined by the heat of solution of the overlayer and substrate 
(a)

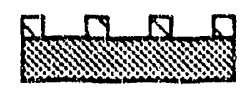

(b)

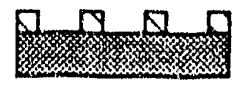

(c)

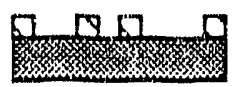

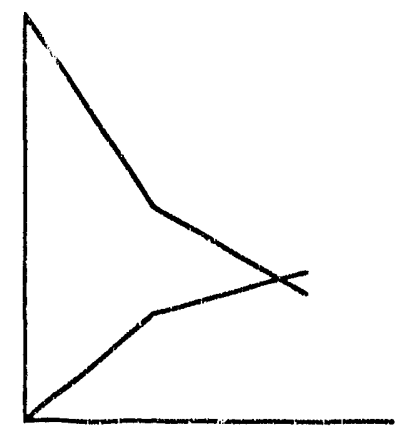
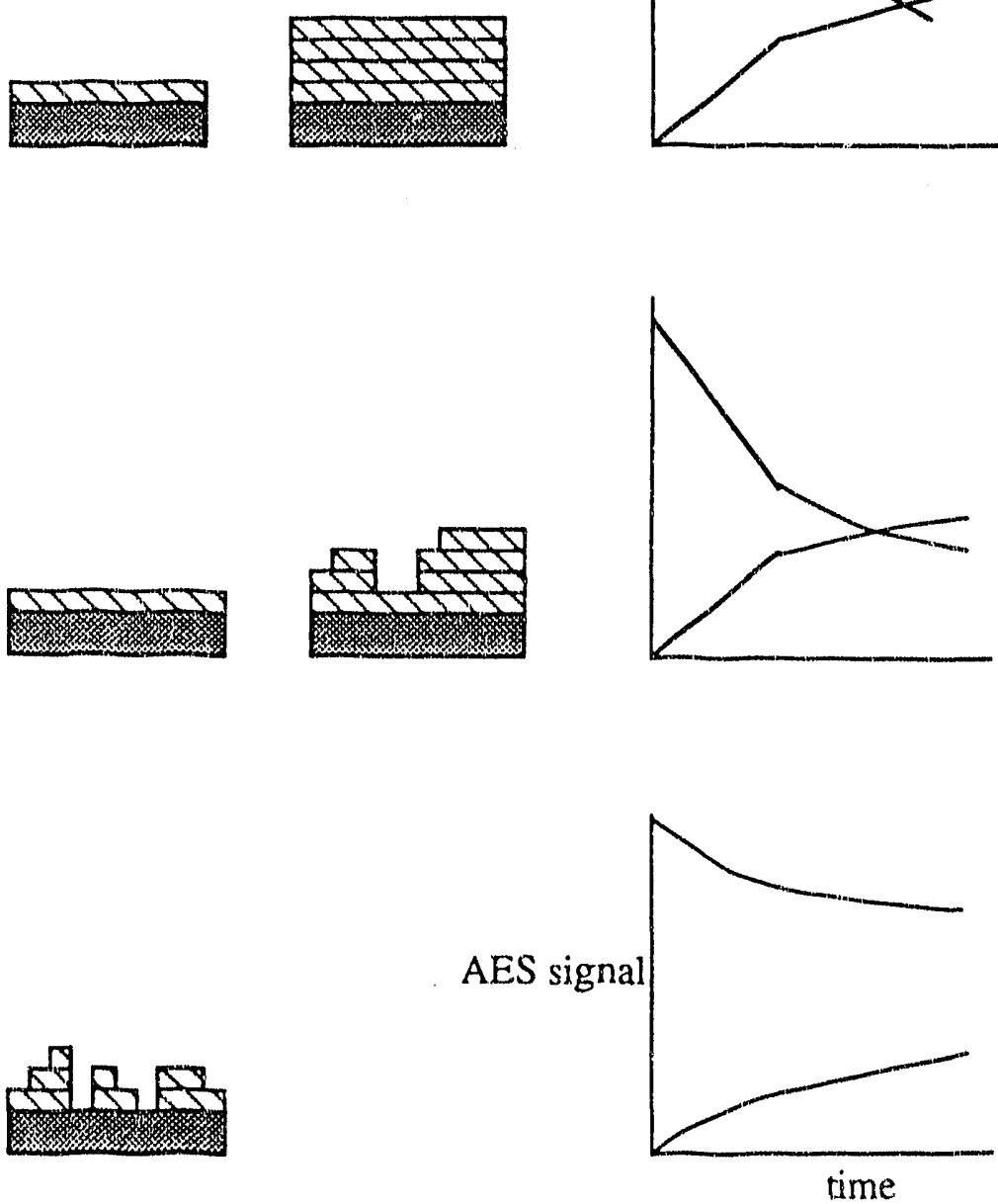

Figure 4.1: Schematic representations of expected Auger plots of the three distinct growth modes: (a) Frank Van der Merwe, (b) Stranski-Krastanov and (c) Volmer-Weber growth modes. 
material. The structural component depends on the lattice misrnatch between the overlayer and substrate crystal structures. In cases where a layer-by-iayer growth mode is seen, often thin metal films grow with pseudomorphic $(1 \times 1)$ structures when the lattice mismatch between the overlayer and substrate crystal structures is less than $10 \%{ }^{4}$. This pseldomorphic growth does not continue indefinitely since with increasing film thickness, the lattice strain in the growing film increases. At a critical thickness which is inversely related to the lattice misinatch betwesin the overlayer and substrate material, misfit dislocations are formed at the interface to relieve the mismatch strain ${ }^{5}$.

Growth is generally governed by kinetic constraints. For heteroepitaxy of a metal overlayer on a metal substrate with similar melting point, the temperature range required for the growth of flat, atomically sharp interfaces is narrow. For deposition rates between 0.1 and $1 \mathrm{ML} / \mathrm{s}$, the substrate must be heated to a temperature that is between $30-40 \%$ of the melting temperature 6 . These elevated temperatures are necessary because at lower remperatures, surface diffusion of the impinging molecules is too slow to allow the ordering into atomically flat layers. In contrast to the structural disorder observed at low substrate temperatures, chemical disorier is observed at the interface at higher temperatures. This chemical disorder results from the possibility of bulk interdiffusion at - higher temperatures.

\subsection{Determination of Growth Modes using Auger Spectroscopy}

If a Frank-Van der Merwe growth mode is experimentally realized and one plots the absolute peak intensities due to Auger transitions of the substrate and overlayer, one should find sequences of linear regions with decreasing slopes. As the overlayer becomes 
thicker, decreasing numberis of Auger electrons of the substrate originate within the escape depth of the surface. Thus the intensity of the substrate Auger peaks decrease. The expected plots were schematically shown in figure 4.1(a).

The linear change in Auger intensities can be understood using the equations below. In layer-by-layer growth, $(n-1)$ adsorbate monolayers are fully completed before the $n$th layer begins to grow. At any coverage $\theta$ in monolayers, such that $(n-1)<\theta<n$, the following expressions can be written.

$$
\theta=\mathrm{n}-1+\varphi \quad \text { where } 0<\varphi<1 \quad \text { eq. i }
$$

This means that a fraction $(\varphi)$ of the surface is covered by $n$ adsorbate layers and the rest of the surface $(1-\varphi)$ is covered with $(n-1)$ adsorbate layers. These two fractions have different magnitudes of contribution to the total Auger signal of the adsorbate. The fraction $\varphi$ contributes $\varphi I_{A n}$ while the remainder of the surface contributes $(1-\varphi) I_{A(n-1)}$ to the total Auger current. The total Auger current $\left(\mathrm{I}_{\mathrm{A}}\right)$ is given below.

$$
\begin{array}{rlr}
I_{A} & =\varphi I_{A n}+(1-\varphi) I_{A(n-1)} & \text { eq. } 2 \\
& =I_{A(n-1)}+\left(I_{A n}-I_{A(n-1)}\right) \varphi & \text { eq. } 3
\end{array}
$$

where

$I_{A n}=$ Auger current due to $n$ adsorbate layers

$I_{A(n-1)}=$ Auger current due to $(n-1)$ adsorbate layers

From eq. 3 , it can be seen that during the completion of the $n$th monolayer, the Auger signal of the adsorbate linearly increases with coverage. The slope is determined by the difference in the Auger current due to the $n$th and $(n-1)$ th layer. As $n$ becomes larger, the difference in the Auger current due to contributions from the $n$th and $(n-1)$ th layer 
decreases and therefore the slope decreases. Similarly, there is a linear decrease in the total Auger current due to the substrate.

The completion of consecutive monolayers manifest themselves by discontinuous changes in the slope of the linear regions. The main problem of determining coverages in this manner is that it is often difficult to find the break points especially at higher coverages. Hence, it is not trivial to correctly assign the completion of various monolayers. This problem is even more severe when higher energy, and therefore less surface sensitive, Auger transitions are considered.

In the case of Volmer-Weber growth, there is an exponential decrease and increase in the intensities of Auger transitions due to the substrate and overlayer respectively. Plots of expected Auger intensities as a function of coverage (deposition time) are shown in figure 4.1(c). A Stranski-Krastanov growth mode manifests itself by a first few linear segments followed by an exponential change in the intensity of Auger peaks.

\subsection{Determination of Desorption Energetics From Thermal Desorption Experiments}

In these thermal desorption experiment, the vaporization rate of an adsorhate is measured as a function of the substrate temperature. We have used desorption studies to probe surface chemistry, determine desorption orders, activation energies for desorption, and heats of sublimation of various adsorbates. The desorption order was determined from the dependence of the temperature at the maximum rate of desorption $\left(T_{p}\right)$ on the initial surface coverage (section 2.3.3). For the case of zero order desorption kinetics, spectra from surfaces with different initial coverages exhibit a common leading edge and $T_{p}$ increases with increasing coverage. A common leading edge indicates that the desorption 
rate is independent of the surface coverage. A plot of In rate against $1 / \mathrm{T}$ has a slope of $-E_{d} / R T$. It has been shown that for non complex sublimation systems, the zero order activation energy for desorption into vacuum is equal to the heat of sublimation obtained under equilibrium conditions.

When first order kinetics are observed, $T_{p}$ is independent of coverage and when second order kinetics are followed, $T_{p}$ decreases with increasing coverage. In this work, when first order kinetics are observed, the activation energies of desorption are determined using Redhead's plot of first order activation energies of desorption as a function of $T_{p}$ (figure 2.15). This plot assumes that the pre-exponential factor is $10^{13} \mathrm{sec}^{-1}$ and that both the pre-exponential factor and desorption energy are coverage independent. These assumptions are sometimes invalid, in which case they will lead to errors in the first order activation energies reported ${ }^{7}$.

\subsection{Growth of Cobalt Overlayers on Clean Molybdenum Surfaces}

\subsubsection{Auger Studies}

The Auger uptake curve of cobalt on molybdenum was used to determine coverages (figure 4.2). The experiment was done in the following manner. Cobalt was deposited on the crystal held at room temperature. After each deposition, the crystal was annealed at $870 \mathrm{~K}$ for one minute. After this annealing treatment a $(1 \times 1)$ diffraction partern was observed (chapter 5). The first monolayer grew in a layer-by-layer fashion as documented by a break in the Auger uptake curve. From figure 4.2, the monolayer coverage is determined to occur at this break. For cobalt coverages greater than one monolayer, the 


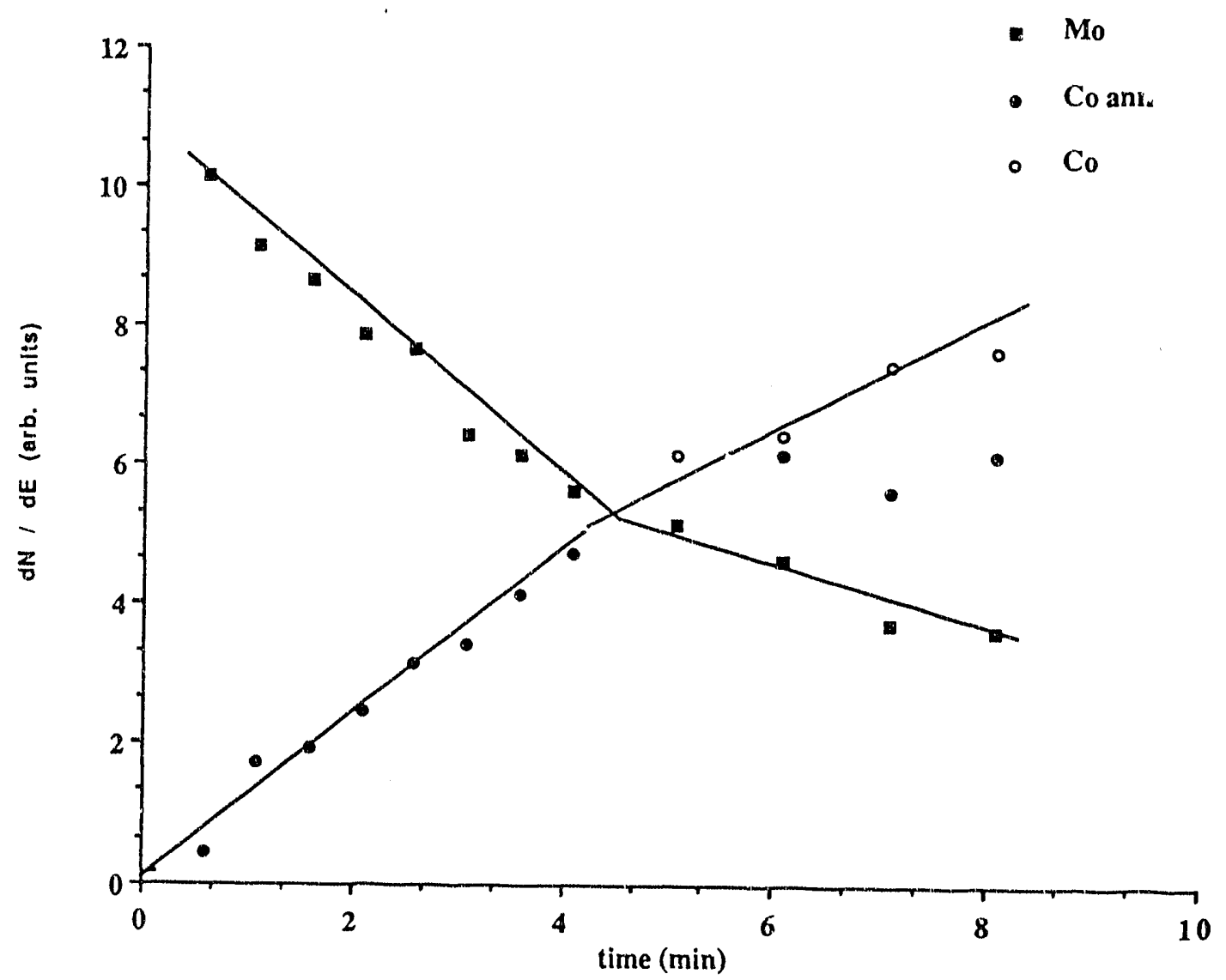

Figure 4.2: Auger uptake curve of cobalt on $\mathrm{Mo}(100)$. 
$\mathrm{Co} /$ Mo Auger ratio decreased significantly after annealing. This decrease is attributed chiefly to the formation of three dimensional cobalt islands at higher cobalt coverages (ie. $\theta_{\mathrm{Co}} \geq 1 \mathrm{ML}$ ). At coverages less than one monolayer, there is no effect of annealing on the $\mathrm{Co} / \mathrm{Mo}$ ratio below the desorption ternperature of cobalt.

A Stranski-Krastanov growth mode is observed for the growth of cobalt on Mo(100). The observed critical thickness is one monolayer. The factor most limiting this critical thickness is the large lattice mismatch of this system (section 5.4). The growth of one monolayer of cobalt grows in a quasi layer-by-layer fashion can be understood using thermodynamic arguments. It is favorable to completely cover the refractory molybdenum surface which has a higher surface free energy $\left(2.91 \mathrm{~J} / \mathrm{m}^{2}\right.$ (calculated at $\left.T=0 \mathrm{~K}\right)$ ) with the lower surface free energy cobalt overlayer $\left(2.52 \mathrm{~J} / \mathrm{m}^{2}\right.$ (calculated at $\left.\left.\mathrm{T}=0 \mathrm{~K}\right)\right)^{3}$. The high surface free energy of metals such as molybdenum or tungsten makes it possible to use these metals as substrates for the epitaxial growth of a few monolayers of group three transition metals. (The iarge difference between the surface free energies of refractory metals and group three transition metals ensures that the interfacial energy term is negligible at least for the first few monolayers.)

The epitaxial cobalt monolayer is strained. Whin multilayers are deposited, the lattice strain increases to the point that the lowering of the surface free energy of the system by covering the molybdenum surface with cobalt can no longer compensate for the lattice strain developed in the pseudomorphic cobalt overlayer. This leads to the breakdown of pseudomorphic growth and resulting island formation. Cobalt overlayers were also deposited on $\mathrm{Mo}(910), \mathrm{Mo}(911)$ and $\mathrm{Mo}(28,4,1)$ surfaces. Using AES, similar growth modes were observed on the vicinal surfaces as on the flat (100) surface. Again, a Stranski-Krastanov growth mode was observed with only the first monolayer growing in a layer-by-layer fashion. Unlike that observed on the flat (100) surface, pseudomorphic (1 x 1) cobalt overlayers were not formed on the vicinal surfaces. The structures observed 
on these surfaces are discussed in chapter 5.

Berlowitz and Goodman ${ }^{8}$ have studied the growth of nickel overlayers on tungsten single crystals. This is a comparable system since tungsten and molybdenum have similar lattice constants $(W=3.16 \AA, M o=3.15 \AA$, on the $(100)$ surfaces) and high surface free energies. Both cobalt and nickel have face centered cubic structures with similar lattice constarits ( $\mathrm{CO}=2.51 \AA, \mathrm{Ni}=2.49 \AA$, on the (111) surface) and surface free energies. Thiy of served that only the first r.onolayer grew in a two dimensional fashion on W(100) and $W(110)$ surfaces following the deposition of nickel and subsequent annealing. Layer-by-layer growth was not observed for multilayers.

\subsubsection{Carbon Monoxide Desorption from Cobalt Overlayers on Molybdenum Surfaces}

CO chemisorption can be used to probe surface chemistry since different surfaces have different $\mathrm{CO}$ binding energies and abilities for $\mathrm{CO}$ dissociation. $\mathrm{CO}$ chemisorption on clean Mo(100) surfaces have been extensively studied and three desorption states have been fourd ${ }^{9-12}$. For the stepped and kinked molybdenum surfaces, we did not find significant changes in the $\mathrm{CO}$ desorption spectra that could be attributed to the presence of steps or kinks. A fraction of the $\mathrm{CO}$ is molecularly desorbed and the desorptiun temperature from this state has been reported to vary from $270 \mathrm{~K}$ to $490 \mathrm{~K}$ on $\mathrm{Mo}(100)$. Dissociatively adsorbed $\mathrm{CO}$ desorbs around $1020 \mathrm{~K}$ and $1240 \mathrm{~K}$ after recombination. It is not understood why two recombination states are seen since HREELS studies report only one metal carbon stretch for both states. There have been comparatively few chemisorption studies on cobalt single crystal surfaces. These surfaces are difficult to prepare because bulk cobalt undergoes a phase transition from a hexagonal close packed structure to a face centered cubic structure around $700 \mathrm{~K}$. Papp has shown usitig ultraviolet photoelectron 
spectmscopy that on the more corrugated $\operatorname{Co}(11 \overline{2} 0)$ surface, both molecular $\mathrm{CO}$ and atomic oxygen from dissociatively adsorbed $\mathrm{CO}$ coexist above $300 \mathrm{~K}$. The adsorbed carbon could not be detected. On the less corrugated $\mathrm{Co}(10 \overline{10})$ and $\mathrm{C} \sim(0001)$ surfaces no dissociation product were observed ${ }^{13}$. Polycrystalline cobalt surfaces dissociate $\mathrm{CO}$ above room temperature 14,15 leaving carbon and oxygen atoms on the surface. In this section, the results of $\mathrm{CO}$ desorption studies from molybdenum surfaces covered with different amounts of cubalt are used to further probe the morphology of the cobalt overlayer. In these experiments, cobalt was svaporated onto molybdenum surfaces. The surface was exposed to a saturation CO dose of $\sim 0.3 \mathrm{~L}\left(1 \mathrm{~L}=10^{-6} \mathrm{Torr} \cdot \mathrm{sec}\right)$ and then heated at a rate of about $30 \mathrm{~K} / \mathrm{sec}$. The rate of $\mathrm{CO}$ desorption was monitored by mass spectrometry. In figure 4.3, typical carbon monoxide desorption traces from the $\mathrm{Mo}(28,4,1)$ surface with various cobalt coverages are shown. The lowest temperature $(\sim 420 \mathrm{~K})$ desorption peak is due to molecular CO desorption. The two higher temperature states, $\beta_{1}$ and $\beta_{2}$ result from $C O$ dissociation followed by recombination of carbon and oxyge? atorns and subsequent desorption. The highest temperature desorption peak and the high temperature shoulder on the $\beta_{1}$ peak are the most sensitive to the cobalt coverage. For cobalt coverages above $1 \mathrm{ML}$, the temperature at the maximum rate of $\mathrm{CO}$ desorption from the $\beta_{2}$ state is about $1160 \mathrm{~K}$. This is significantly lower than the temperature at the maximum rate of $\mathrm{CO}$ desorption from the corresponding state on the clean surface. Cobalt desorbs from the $\beta_{2}$ state on the clean surface around $1320 \mathrm{~K}$. On surfaces covered with one monolayer or more of cobalt CO desorption is complete by $1260 \mathrm{~K}$, well below the temperature at which the rate of cobalt desorption itself becomes significant (figures 4.7 , 4.8 -4.13). The $\beta_{2}$ peak is relatively narrow suggesting that $\mathrm{CO}$ desorbs from one type of site, namely sites provided by the cobalt overlayer. This means that even if cobalt in the second layer has formed three dimensional islands, the first cobalt monolayer is still relatively flat and fully covers the molybdenum surface. 


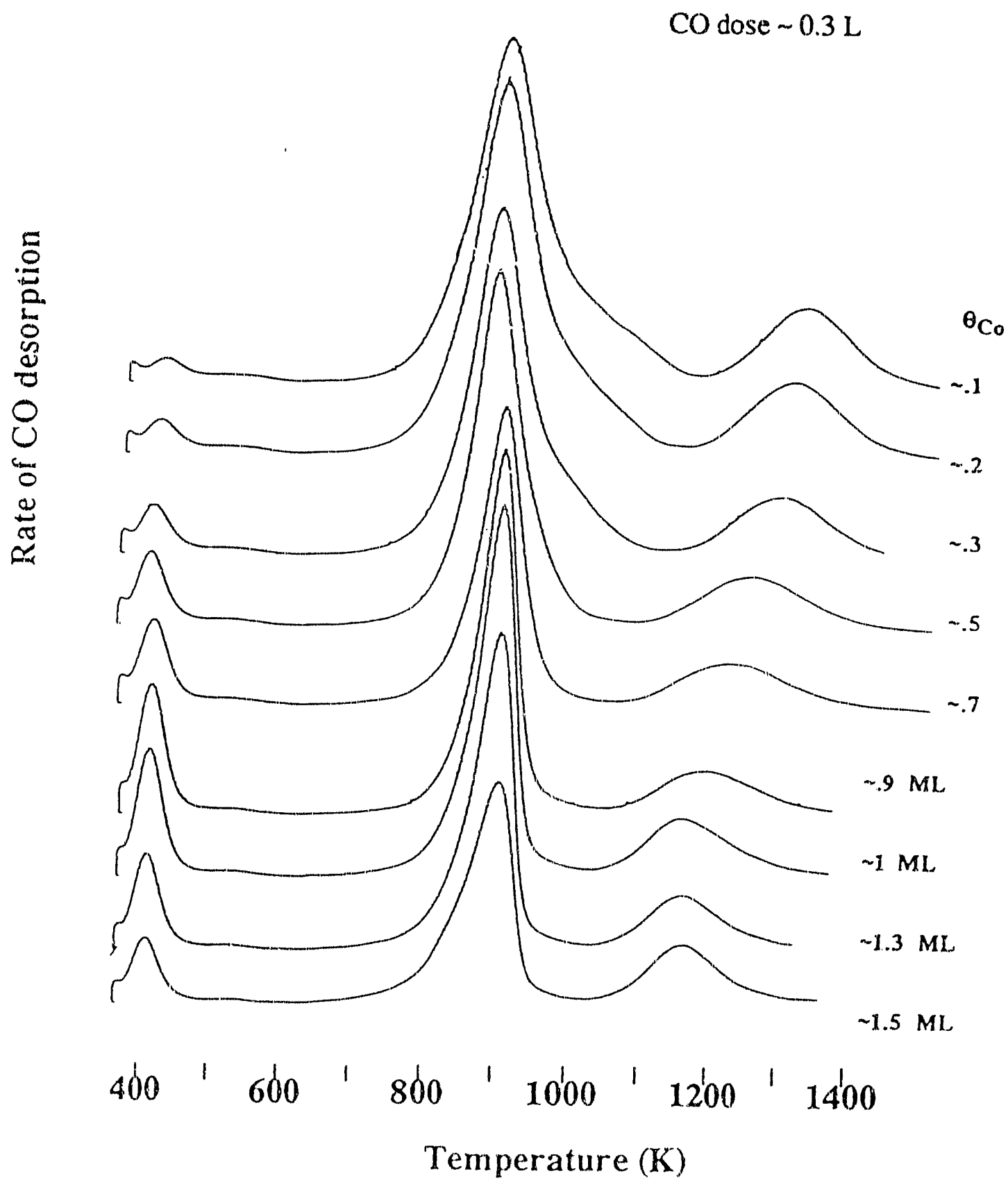

Figure 4.3: $\quad C O$ desorption from cobalt overlayers on $M o(28,4,1)$ surfaces. 
As the coilt coverage is decreased irom $1 \mathrm{ML}$ to $0.1 \mathrm{ML}$, the temperature at the maxinum rate of desorpuvin of the $\beta_{2}$ peak increases and the full width at half of the peak maximum first increases and then decreases. The width of this peak is a maximum at cobalt coverages close to half of a monolayer. One possible explanation of these observations is that in the cobalt submonoliayer regime, $\mathrm{CO}$ is desorbing from two unresolvable states. These states are dup; to exposed cobalt and molybdenum sites. The uppermost deso.ption trace is typical of CO desorption from clean $\operatorname{Mo}(23,4,1)$ surfaces. The increasing desorption temperature can alternatively be attributed to a shift in $\mathrm{CO}$ binding energy with cobalt coverage. It should be noted that at the lowest cobalt coverages, the onset of cobalt desorption begins before carbon monoxide desorption is complete.

As the cobalt coverage decreases, the high temperature shoulder on the $\beta_{1}$ peak characteristic of $\mathrm{CO}$ desorption from clean molybdenum surfaces appears. This shoulder is not seen on surfaces for which the initial cobalt coverage is greater than one monolayer, again indicating that the first cobalt monolayer grows approximately in a layer-by iayer fashion.

\subsection{Growth of Cobalt Overlayers on Adsorbate Covered Surfaces}

\subsubsection{Auger Studies}

Cobalt was deposited on the different sulfur overlayers on flat and vicinal molybdenum surfaces. Figure 4.4 is a plot of the intensity of the $\mathrm{Mo}(\mathrm{MNN}) 186 \mathrm{eV}$, $\mathrm{S}(\mathrm{LMM}) 152 \mathrm{eV}$ and $\mathrm{Co}(\mathrm{LMM}) 775 \mathrm{eV}$ Auger transitions versus cobalt deposition time for 


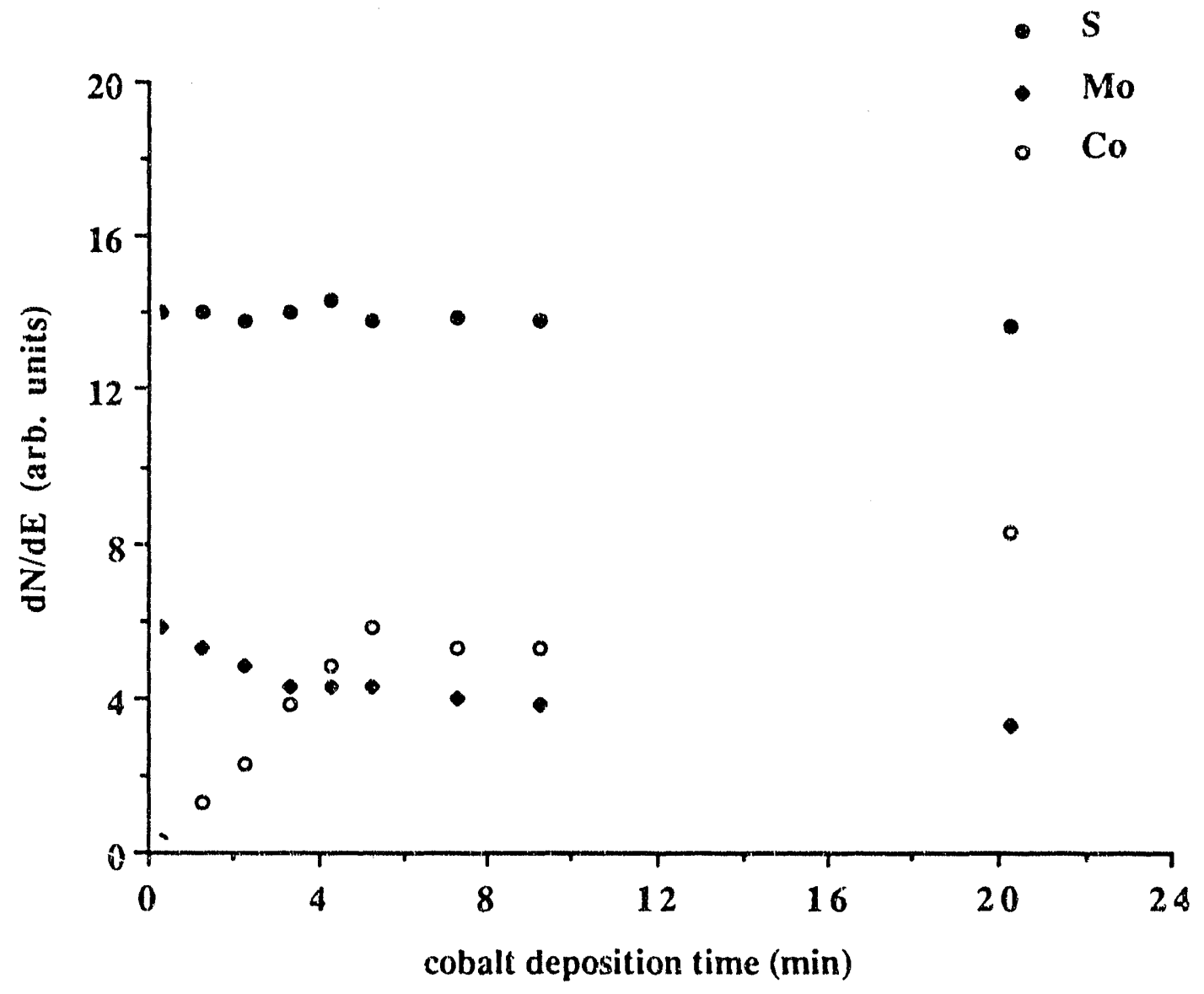

Figure 4.4: Plot of the intensity of the Mo (MNN) $186 \mathrm{eV}, \mathrm{S}$ (LMM) $152 \mathrm{eV}$ and $\mathrm{Co}$ (LMM) $775 \mathrm{eV}$ Auger transitions as a function of cobalt deposition time on the $M o(100)-c(2 \times 2) S$ surface. 
the deposition of cobalt on the $\operatorname{Mo}(100)-c(4 \times 2) S$ surface. After evaporation of more than three monolayers of cobalt, the sulfur Auger intensity is decreased by less than $15 \%$. If a monolayer of cobalt was covering the sulfur overiayer, based on the escape depth of the $\mathrm{S}(\mathrm{LMM}) 152 \mathrm{eV}$ electrons, a $40-60 \%$ attenuation of the signal would be expected. I his lack of attenuation of the sulfur Auger intensity is also seen after the deposition of cobalt on other sulfur covered molybdenum surfaces. This is true even when the crystal is held at $150 \mathrm{~K}$. Observation of diffraction pattems before annealing show that the surfaces are disordered. After annealing at $870 \mathrm{~K}$, various diffraction patterns which are discussed in chapter 5 are observed. The fact that the sulfur Auger intensity does not decrease significantly indicates that sulfur "floats out" on top of the cobalt overlayer. Further proof that the topmost layer is composed of sulfur is provided by the stability of these overlayers. Comparatively little carbon and oxygen accumulated on these surfaces after exposure to one Torr of air at $300 \mathrm{~K}$ for sixteen hours in the high pressure cell. The Auger spectra before and after air exposure are shown in figure 4.5. After similar exposure of either the clean $\mathrm{Mo}(100)$ surface or a cobait covered $\mathrm{Mo}(100)$ surface to air, sigraificant amounts of graphite and oxygen, presumably from adsorption of hydrocarbons, $\mathrm{CO}$ and $\mathrm{CO}_{2}$ are detected on these surfaces. It is known that when $M \circ(100)$ surfaces are covered with one monolayer of sulfur, they remain "clean" after air exposure ${ }^{16}$.

Contrasting results were obtained when cobalt was deposited on carbon covered surfaces $\left(\theta_{C}=1 \mathrm{ML}\right)$. In these experiments a decrease in the iniensity of the $\mathrm{C}(\mathrm{KLL}) 272 \mathrm{eV}$ transition was observed. Carbon overlayers were formed by decomposing ethylene on the molybdenum surfaces. This necessarily leads to approximately equal coverages on both the front and back faces of the crystal. Since cobalt is only deposited on one face, monitoring the back face provides the change in carbon coverage from reaction with background gases. Throughout the experimeict, the carbon coverage on the back face remains constant. Therefore the decrease in carbon Auger 


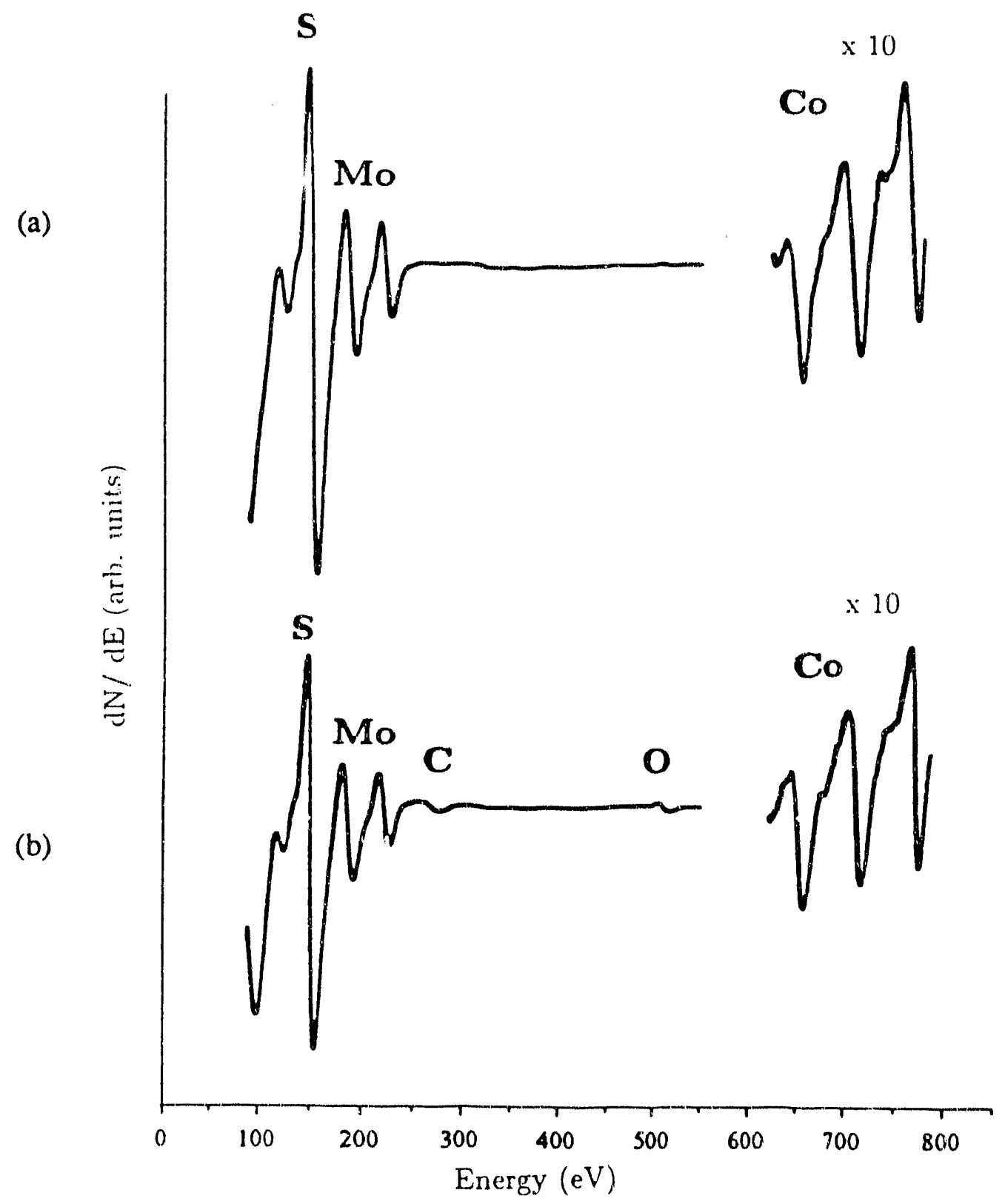

Figure 4.5: Auger spectra (a) before and (b) after air exposure of the $\mathrm{Co} / \mathrm{Mo}(100)-c(2 \times 2) \mathrm{S}$ surface. 
intensity on the front face is due to covering of this overlayer with cobalt and not to reaction with ambient gases. Carbon therefore does not "float out" on top of the growing cobalt overlayer.

The surface segregation behavior of sulfur can be qualitatively understood. In general, $S_{8}$ has a lower surface free energy $\left(0.06 \mathrm{~J} / \mathrm{m}^{2}\right.$ at the melting point $\left.{ }^{17}\right)$ than metals. Even though sulfur adsorbed on a surface will have a higher surface free energy than $S_{8}$, it will still be lower than the surface free energy of either cobalt or molybdenum. Therefore a structure in which sulfur is in the topmost layer is compatible with surface energy considerations. It was not possible to find a value of the surface free energy of carbon in the form of a carbidic overlayer on a metal.

Egelhoff and Steigerwald have studied the role of several adsorbed gases in metal on metal epitax $y^{18}$. Their list of gases included molecularly adsorbed $\mathrm{CO}$ and $\mathrm{H}_{2} \mathrm{O}$ and dissociatively adsorbed $\mathrm{H}, \mathrm{O}, \mathrm{N}, \mathrm{C}$ and $\mathrm{S}$. With the exception for carbon and nitrogen, ihese authors have found that these adsorbed gases generally tend to "float out" on top of the deposited metal overlayer. In the epitaxial growth of copper on $\mathrm{Ni}(100)-\mathrm{c}(2 \times 2) \mathrm{O}$ or nickel on $\mathrm{Cu}(100)-\mathrm{c}(2 \times 2) \mathrm{O}$ at $80 \mathrm{~K}$, the preadsorbed oxygen overlayer "floats out" on top of the growing copper or nickel film respectively.

\subsubsection{Carbon Monoxide Thermal Desorption}

A preadsorbed saturated sulfur overlayer completely blocks sites for $\mathrm{CO}$ chemisorption from molybdenum surfaces ${ }^{19}$. To examine the effect of co-adsorbed cobalt on the sulfur covered surface, one monolayer of cobalt was deposited on the $\operatorname{Mo}(28,4,1)-c(2 \times 2) S$ surface prior to adsorption of $\sim 0.3 \mathrm{~L}$ of $\mathrm{CO}$. The CO capacity of the surfaces is substantially reduced from the CO capacity on either the clean or cobalt covered surfaces. The capacity of the surfaces for $\mathrm{CO}$ chemisorption compares well with 


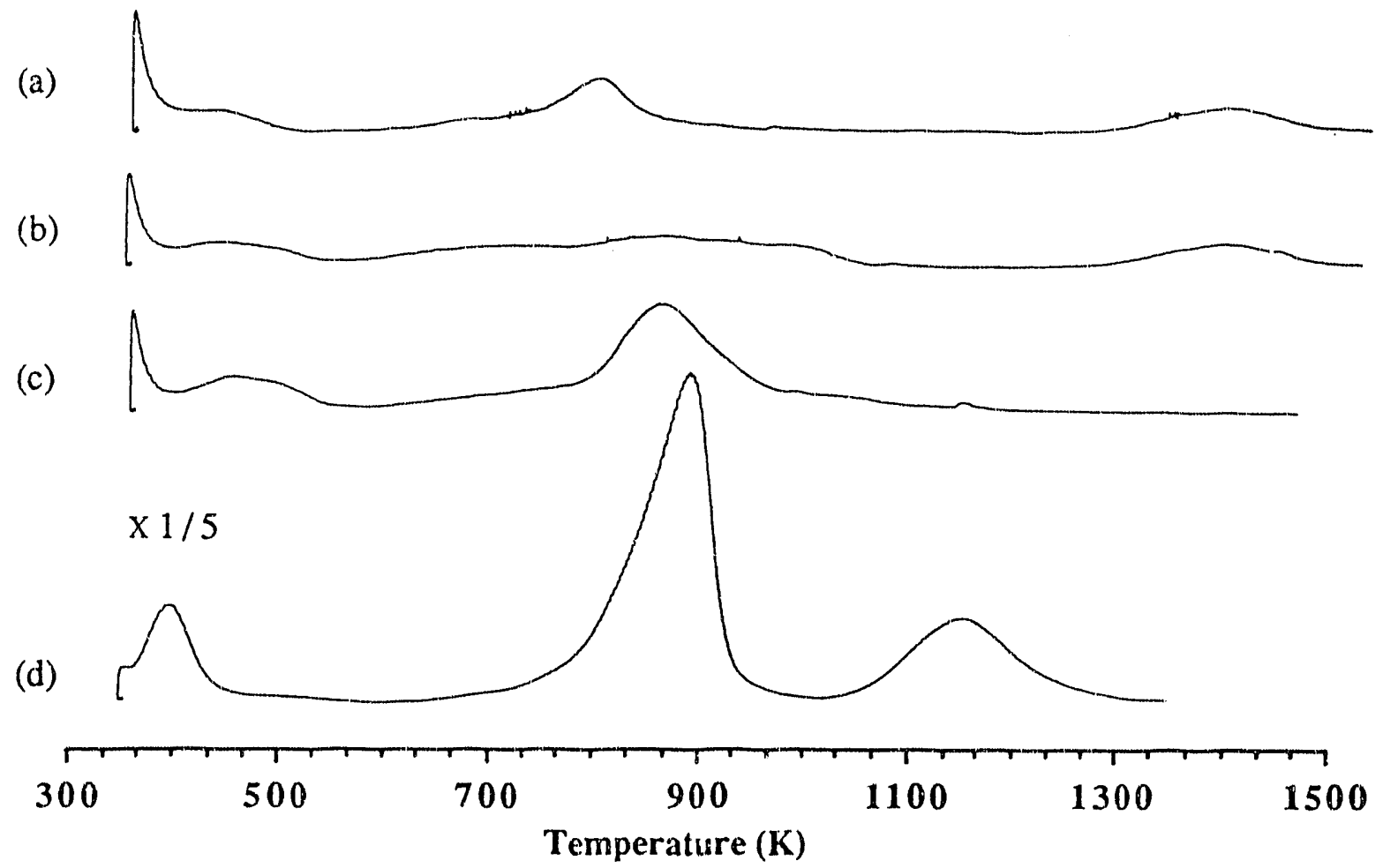

Figure 4.6: $\quad 0.3 \mathrm{~L} \mathrm{CO}$ desorption from the following surfaces:

(a) 1.3 ML Co $/ \mathrm{Mo}(28,4,1)-\mathrm{c}(2 \times 2) \mathrm{S}$ (annealed at $870 \mathrm{~K})$,

(b) 1.3 ML Co/ $\mathrm{Mo}(28,4,1)-c(2 \times 2) S$ (unannealed),

(c) $\operatorname{Mo}(28,4,1)-c(2 \times 2) S$ and

(d) $1.3 \mathrm{ML} \mathrm{Co} / \mathrm{Mo}(28,4,1)$. 
the capacity on sulfur covered surfaces. This corroborates the Auger studies described above which suggest that when cobalt is deposited on sulfur covered surfaces, the sulfur overlayer migrates to the top of the cobalt overlayer.

\subsection{Desorption of Cobalt Overlayers from Mo(100) Surfaces}

Except when otherwise stated, in the following desorption experiments, cobalt is adsorbed on the $\operatorname{Mo}(100)$ crystal at room temperature. On both the clean and carbon covered $\left(\theta_{C}=1 \mathrm{ML}\right) \mathrm{Mo}(100)$ surfaces, the first cobalt monolayer desorbs with first order kinetics. The temperature at the maximum rate of desorption $\left(T_{p}\right)$ is about $1313 \mathrm{~K}$, and in the submonolayer regime, it is independent of the cobalt coverage. Above one monolayer, $T_{p}$ increases and zero order kinetics are observed. Thermal desorption spectra of cobalt from the clean $\operatorname{Mo}(100)$ surface are shown in figure 4.7(a).

From the temperature at the maximum rate of desorption, an activation energy of desorption of about $83 \mathrm{kcal} / \mathrm{mol}$ was determined. Cobalt multilayers desorb from molybdenum single crystal surfaces with zero order kinetics. Therefore, from a plot of $\ln ($ rate) against $1 / T$, the activation energy for desorption which in this case should approximate the heat of sublimation (section 4.4) can be determined. An example of such a plot is shown for cobalt multilayers on the clean Mo(100) surfact (figure 4.8 (a)). The experimentally determined value of $\Delta \mathrm{H}_{\text {subl }}$ of cobalt is about $84 \mathrm{kcal} / \mathrm{mol}$.

On a Mo(100) surface covered with $0.5 \mathrm{ML}$ of sulfur, cobalt desorption is slightly different. At submonolayer coverages, first order desorption kinetics are observed and the temperature at the maximum rate of desorption initially decreases slightly from about $1326 \mathrm{~K}$ at a cobalt coverage of $0.3 \mathrm{ML}$ to $1309 \mathrm{~K}$ at a coverage of about $0.5 \mathrm{ML}$, but then 


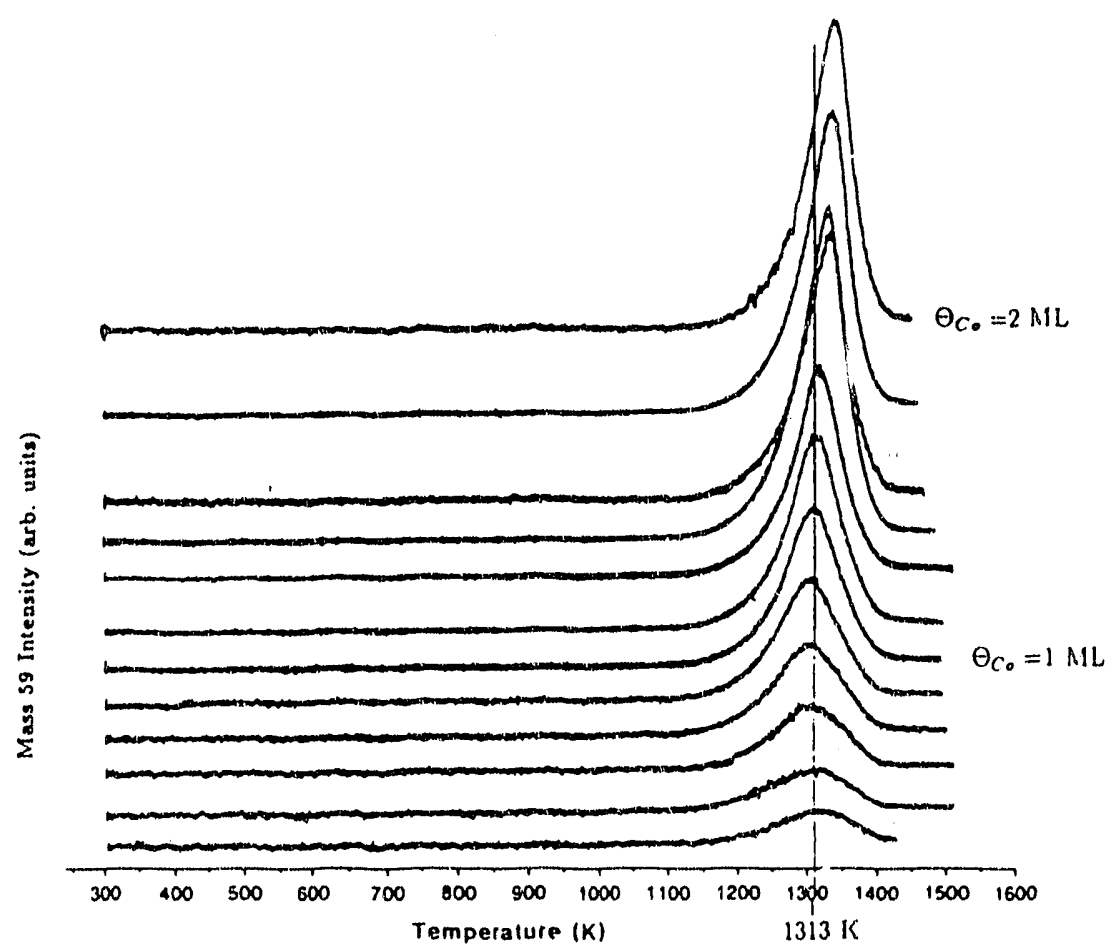

Temperature Programmed Desorption Spectra of Cobalt From Sulfur Covered Mo(100)

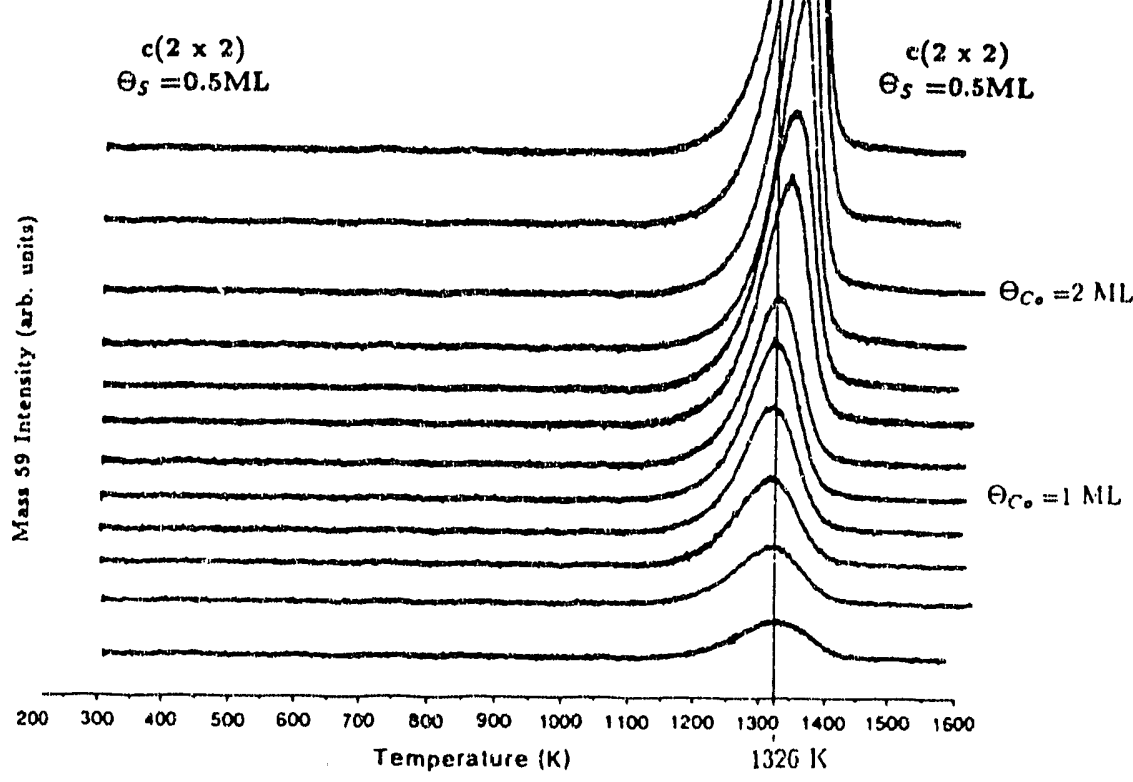

XBL $906-2177$

Figure 4.7: Thermal desorption spectra of cobalt from (a) clean $\operatorname{Mo}(100)$ and (b) $M o(100)-c(2 \times 2) S$ surfaces. 

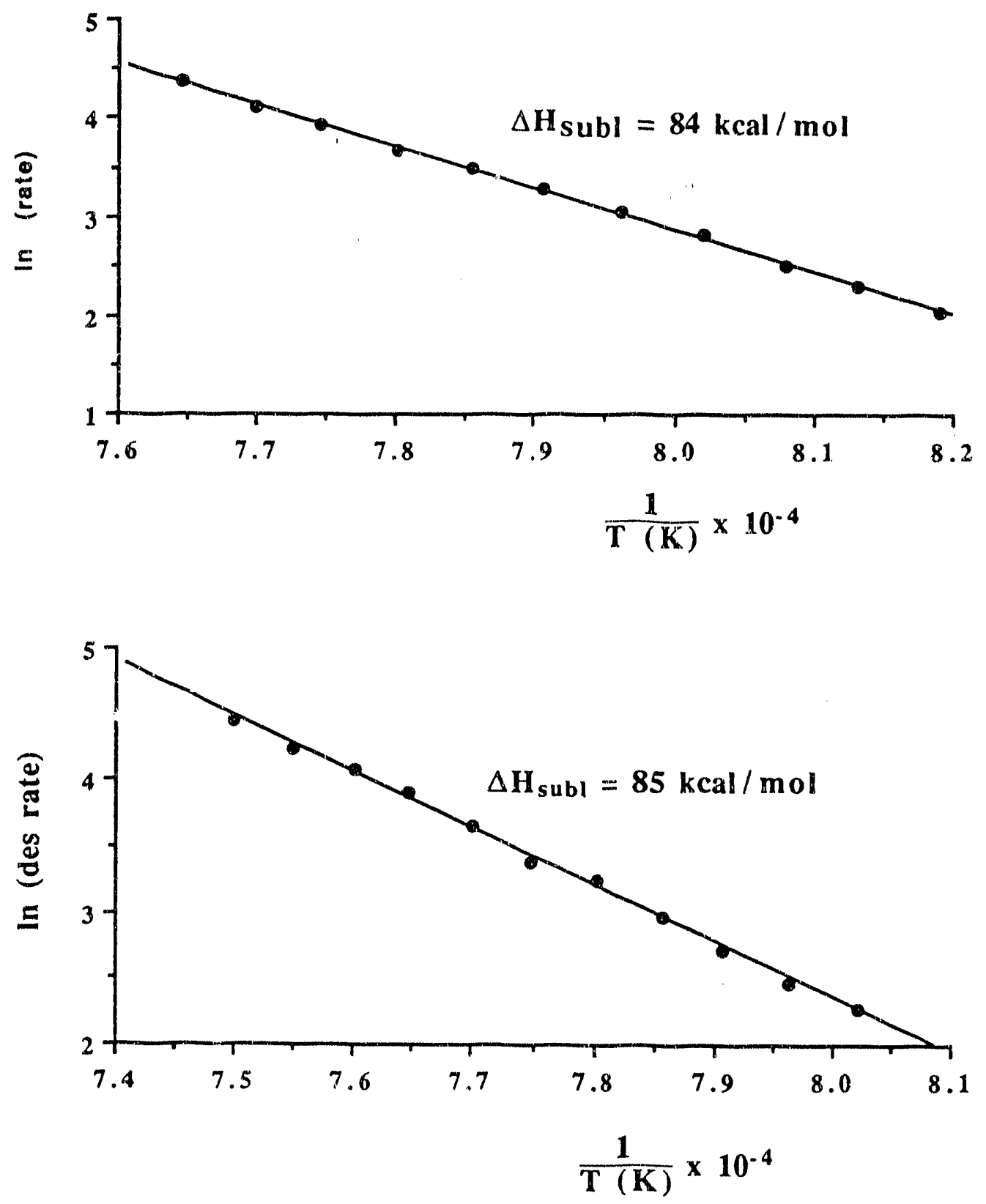

Figure 4.8: Plots of $\ln$ (desurpion rate) against $1 / \mathrm{T}$ for the initial desorption of cobalt multilayers on (a) clean $\mathrm{Mo}(100)$ and (b) $M o(100)$ - $c(2 \times 2) S$ surfaces. 
increases to $1326 \mathrm{~K}$ at a coverage of about $1 \mathrm{ML}$ (figure 4.7 (b)). An activation energy of desorption of cobalt was determined to be about $84 \mathrm{kcal} / \mathrm{mol}$. In section 4.5 , it was shown that when cobalt is deposited on sulfur ccvered molybdenum surfaces, sulfur "floats" on top of the cobalt overlayer. These results now show that this topmost sulfur overlayer does not significantly affect the thermal desorption of cobalt from Mo(100) surfaces. Above one monolayer, zero order desorption kinetics are observed. From a leading edge analysis, $\Delta H_{\text {subl }}$ was determined to be about $85 \mathrm{kcal} / \mathrm{mol}$ (figure 4.7 (b)).

Within experimental error, the first cobalt monolayer is as tightly bound to the molybdenum surface as it is to subsequent cobalt layers. He and Goodman ${ }^{20}$ have looked at ultrathin cobalt films on $\mathrm{Mo}(110)$. Their TDS spectra showed two desorption peaks with activation energies of 82 and $85 \mathrm{kcal} / \mathrm{mol}$ corresponding to multilayer desorption from cobalt clusters and monolayer desorption respectively. Their results also differ from those observed in this work in that they claim that both submonolayer and multilayer desorption exhibit zero order kinetics.

The accepted values for the heat of sublimation is $101 \mathrm{kcal} / \mathrm{mol}$ for face centered cubic cobalt crystals ${ }^{21}$. The lower value obtained in this experiment can be due to several systematic errors which might include inaccuracies in the temperature determination and desorption rate. Also, in many cases, the vaporization rate and consequently the heat of sublimation depends on the surface orientation, defect density and impurity concentration.

\subsection{Desorption of Cobalt Overlayers from Mo(28,4,1) and Mo(911) Surfaces}

Similar to that which is observed on the flat Mo(100) surface, the thermal desorption spectra of cobalt from clean $\mathrm{Mo}(28,4,1)$ and $\mathrm{Mo}(911)$ surfaces are comparable 

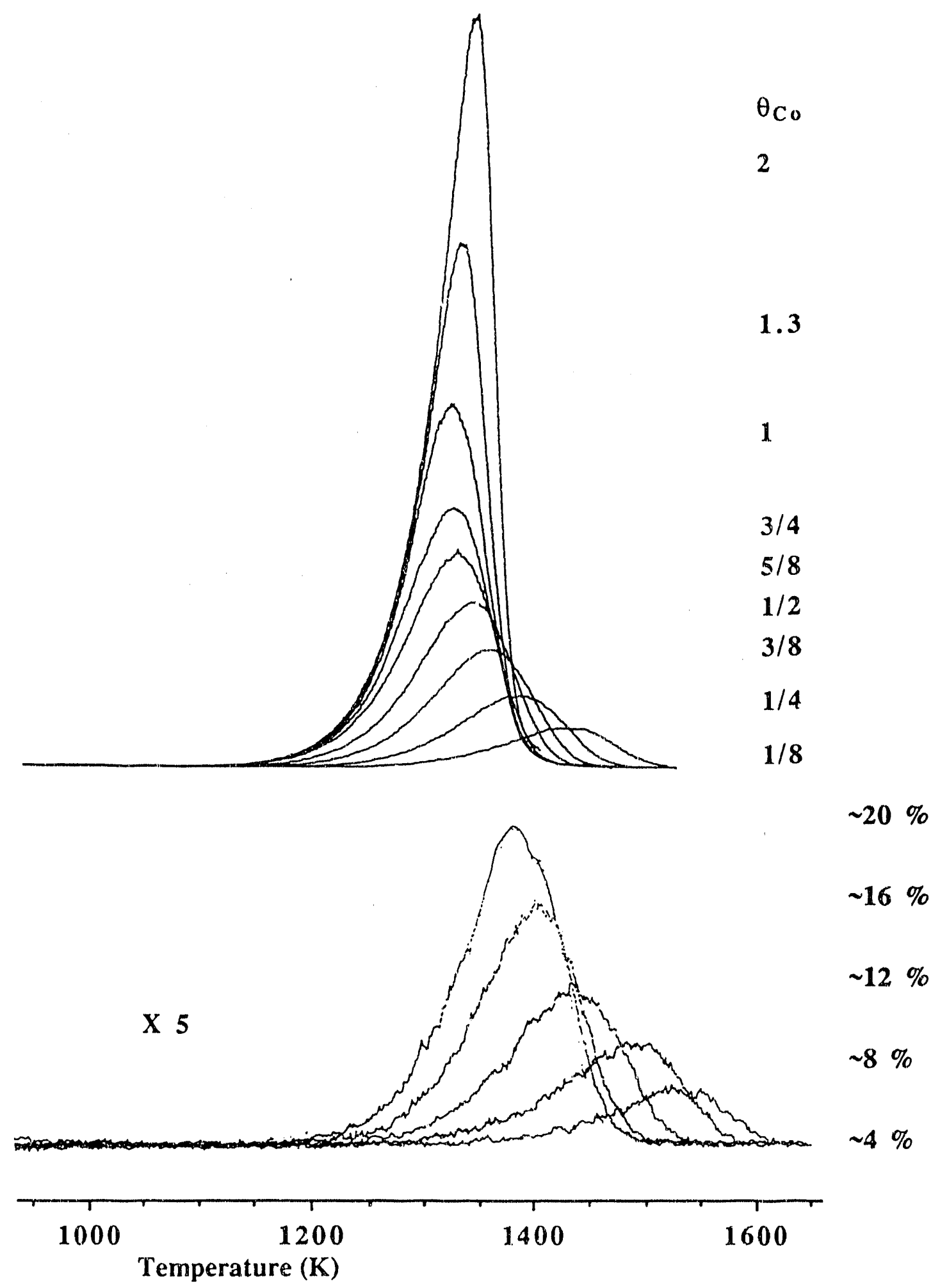

Figure 4.9: Cobalt desorption spectra from clean $\operatorname{Mo}(28,4,1)$ surfaces. 


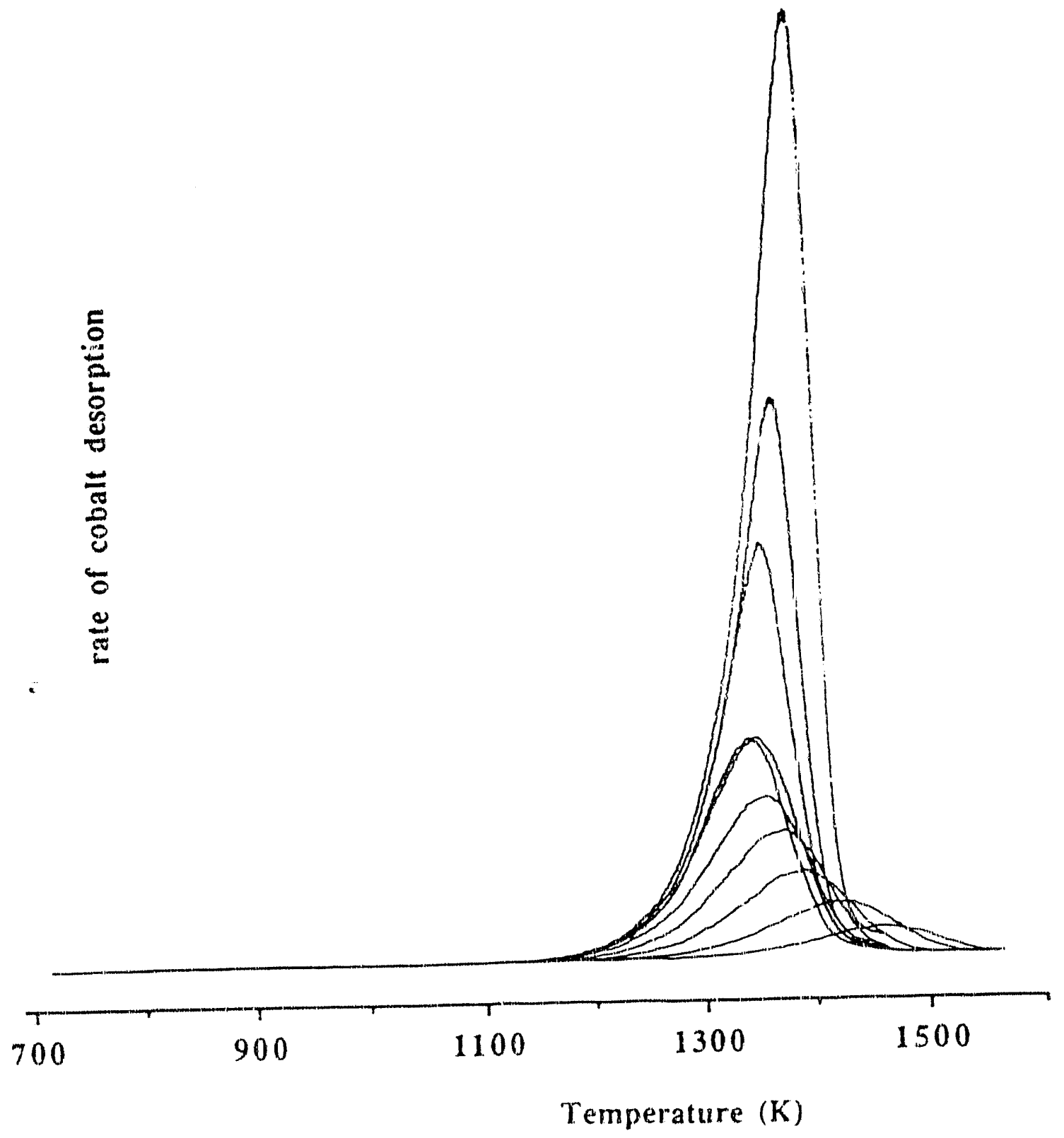

Figure 4.10: Cobalt desorption spectra from $M o(28,4,1)-(1 \times 1)$ C surfaces. $\theta_{C_{0}}=1 / 8,1 / 4,3 / 8,1 / 2,5 / 8,3 / 4,1,2$ and $4 \mathrm{ML}$. 


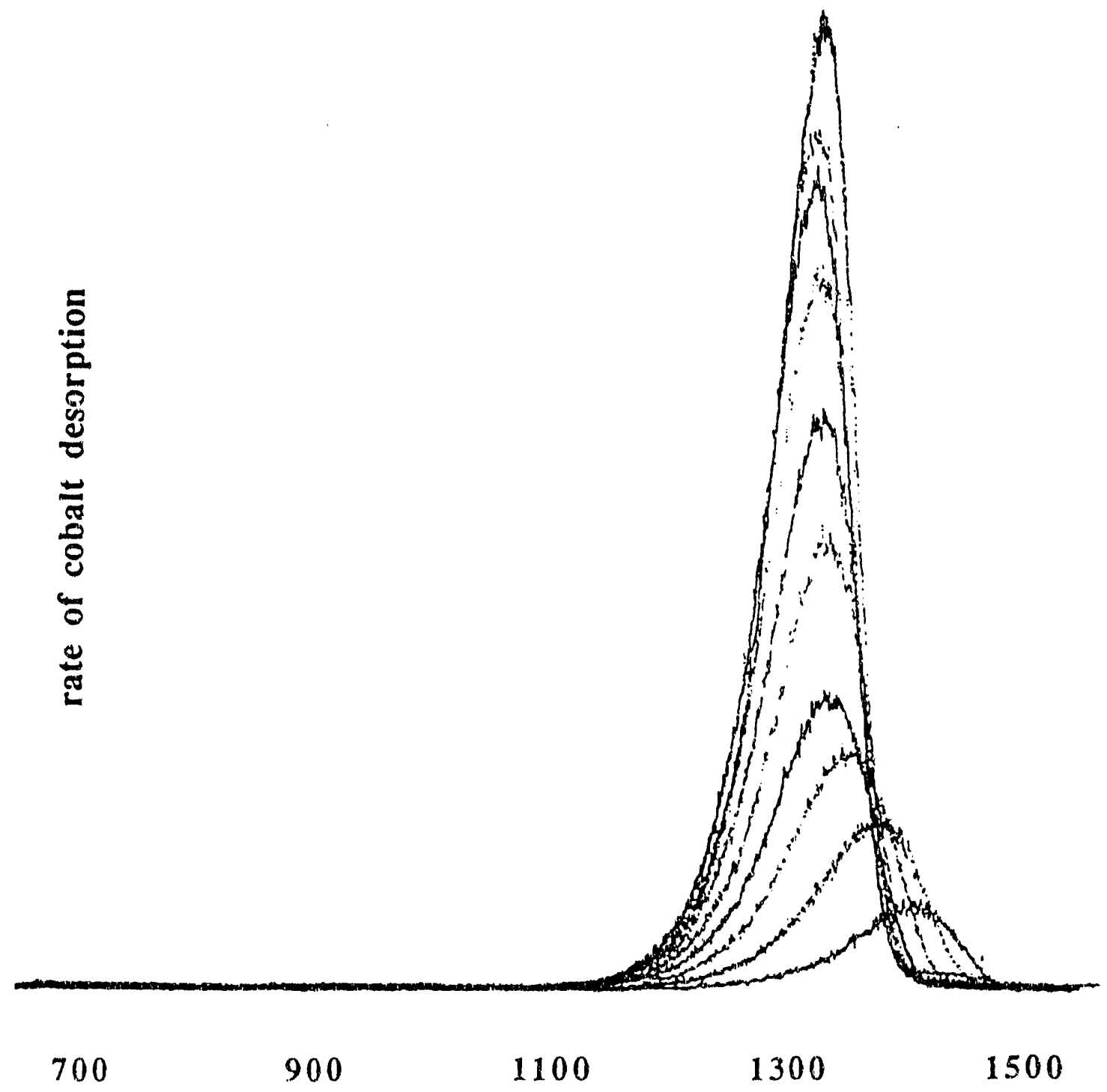

Temperature (K)

Figure 4.11: Cobalt desorption spectra from clean Mo(911) surfaces.

$\theta_{C_{0}}=1 / 8,1 / 4,3 / 8,1 / 2,5 / 8,3 / 4,7 / 8,1,11 / 8$, and $11 / 4 \mathrm{ML}$. 
to the respective desorption spectra from the carbon covered surfaces. These desorption spectra are shown in figures $4.9-4.11$. (Cobalt desorption from the $\mathrm{Mo}(910)$ surface was not investigated). The desorption spectra of cobalt from tho vicinal (100) surfaces are very different from those obtained on the flat $(100)$ surface. On the Mo(28,4,1) surface, a high temperature desorption peak $\left(T_{p} \sim 1530 \mathrm{~K}\right)$ is seen at low coverages $(\sim 0.04 \mathrm{ML})$. As the cobalt coverage increases to one monolayer, the peak desorption temperature decreases to about $1480 \mathrm{~K}, 1430 \mathrm{~K}, 1395 \mathrm{~K}$ and $1375 \mathrm{~K}$ at coverages of approximately $8 \%, 12 \%$, $16 \%$ and $20 \%$ of a monolayer respectively (figure 4.9 ). At a cobalt coverage of about $4 \% \mathrm{ML}, \mathrm{T}_{\mathrm{p}}$ is $\sim 220 \mathrm{~K}$ higher on the kinked $\mathrm{Mo}(28,4,1)$ surface than on the flat $(100)$ surface. In addition, with increasing cobait coverage, the desorption of cobalt is complete at lower temperatures. This is not typical for zero, first or second order desorption kinetics with a fixed activation energy and prefactor. Thie full width of the peaks at half maximum is relatively constant. It is about $160 \mathrm{~K}, 190 \mathrm{~K}, 190 \mathrm{~K}, 170 \mathrm{~K}, 170 \mathrm{~K}$ at coverages of $\sim 4 \%, 8 \%, 12 \%, 16 \%$ and $20 \%$ of a monolayer respectively. Therefore in this coverage regime, the completion of cobalt desorption at lower temperatures with increasing cobalt coverage can not be attributed to the decreasing temperature at the maximum rate of desorption. Between $5 / 8 \mathrm{ML}$ and $1 \mathrm{ML}$, first order desorption kinetics are observed with the temperature at the maximum rate of desorption decreasing slightly from $1330 \mathrm{~K}$ to $1322 \mathrm{~K}$.

The effect of substrate temperature during cobalt deposition was investigated. In a sequence of experiments, low cobalt coverages $\left(1 / 8 \leq \theta_{\mathrm{Co}}(\mathrm{ML}) \leq 1 / 2\right)$ were deposited on the $\mathrm{Mo}(28,4,1)$ surface while the substrate was held at $473 \mathrm{~K}, 573 \mathrm{~K}, 673 \mathrm{~K}$ and $773 \mathrm{~K}$. The molybdenum sample was allowed to cool to about $350 \mathrm{~K}$ before the crystal was linearly heated to desorb the cobalt. In figure 4.12, the observed cobalt desorption traces are shown. Cobalt desorption traces from surfaces initially held at $473 \mathrm{~K}$ or higher exhibit approximate first order desorption kinetics. The temperature at the maximum rate of 


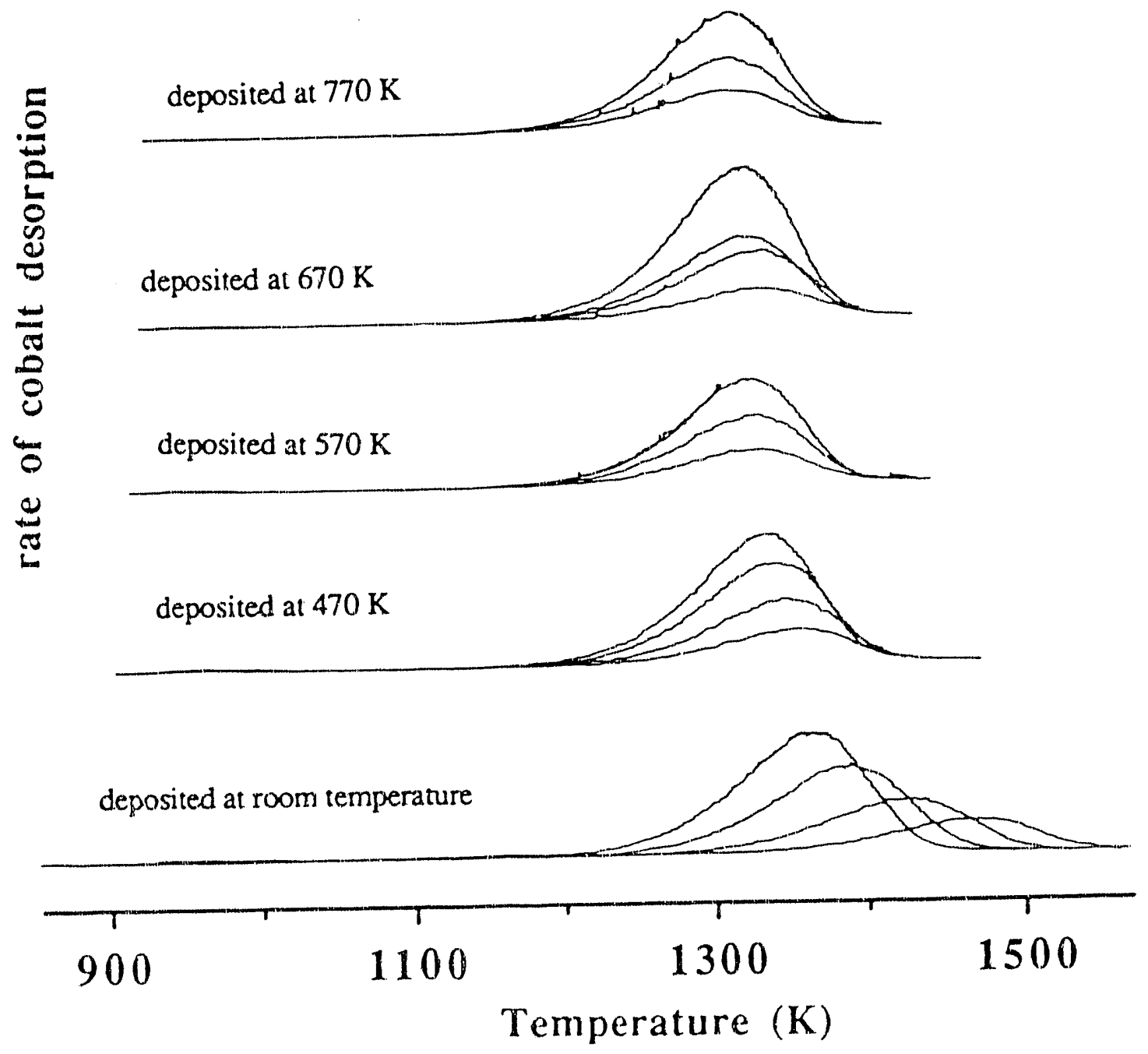

Figure 4.12: Effect of pre-annealing on cobalt desorption from $\operatorname{Mo}(28,4,1)$ surfaces. $\theta_{\mathrm{Co}}=1 / 8,1 / 4,3 / 8$ and $1 / 2 \mathrm{ML}$ 
desorption vary from about $1326 \mathrm{~K}$ for surfaces annealed at $473 \mathrm{~K}$ to about $1309 \mathrm{~K}$ for surfaces annealed at $773 \mathrm{~K}$. These temperatures compare reasonably well with both the temperature at the maximum rate of desorption $(\sim 1330 \mathrm{~K})$ found for cobalt overlayer between coverages of $5 / 8 \mathrm{ML}$ and $1 \mathrm{ML}$ deposited at room temperature and submonolayer cobalt coverages on the flat (100) surface $\left(T_{p}-1313 \mathrm{~K}\right)$.

A preadsorbed $c(2 \times 2) S$ overlayer has a profound effect on cobalt adsorption. Unlike what is observed on the clean and carbon covered $\operatorname{Mo}(28,4,1)$ and $\operatorname{Mo}(911)$ surfaces, on the sulfur covered surface, the cobalt desorption peak shapes are characteristic of first order kinetics (figure 4.13). In the submonolayer regime, $T_{p}$ decreases with increasing cobalt coverage. This suggests lateral repulsions between cobalt and sulfur atoms or a weakening of the Co-Mo bond. This was also seen, though to a much lesser extent on the sulfur covered $\mathrm{Mo}(100)$ surface. The first order activation energy of cobalt desorption decreases from $87 \mathrm{kcal} / \mathrm{mol}$ to $84 \mathrm{kcal} / \mathrm{mol}$ as the cobalt coverage increases from 0.1 ML to 1.0 ML. For cobalt coverages less than $5 / 8 \mathrm{ML}$, the desorption temperature of cobalt is lower on the sulfur covered surfaces than on the corresponding clean $\mathrm{Mo}(911)$ and $\mathrm{Mo}(28,4,1)$ surfaces. However, it is still higher than the desorption temperature of cobalt from the flat $\mathrm{Mo}(100)$ surface.

On the clean and carbon covered vicinal Mo(100) surfaces, $T_{p}$ decreases with increasing cobalt coverages while the full width at half maximum remains constant. On the $\operatorname{Mo}(28,4,1)$ surface, two out of seven atoms are in step or kink positions and on the (911) surface, two out of every nine atoms occupy step positions. We can explain the desorption behavior on the vicinal surfaces by cobalt decoration of the molybdenum step edges. Diffraction results presented in section 5.4 suggest that nucleation of cobalt occurs at step edges. The initial high temperature desorption peaks can be attributed to desorption from steps. Since the atoms coalesce at the steps, their local concentration is higher and lateral repulsions between atoms can exist at low coverages and lead to a considerable decrease in 


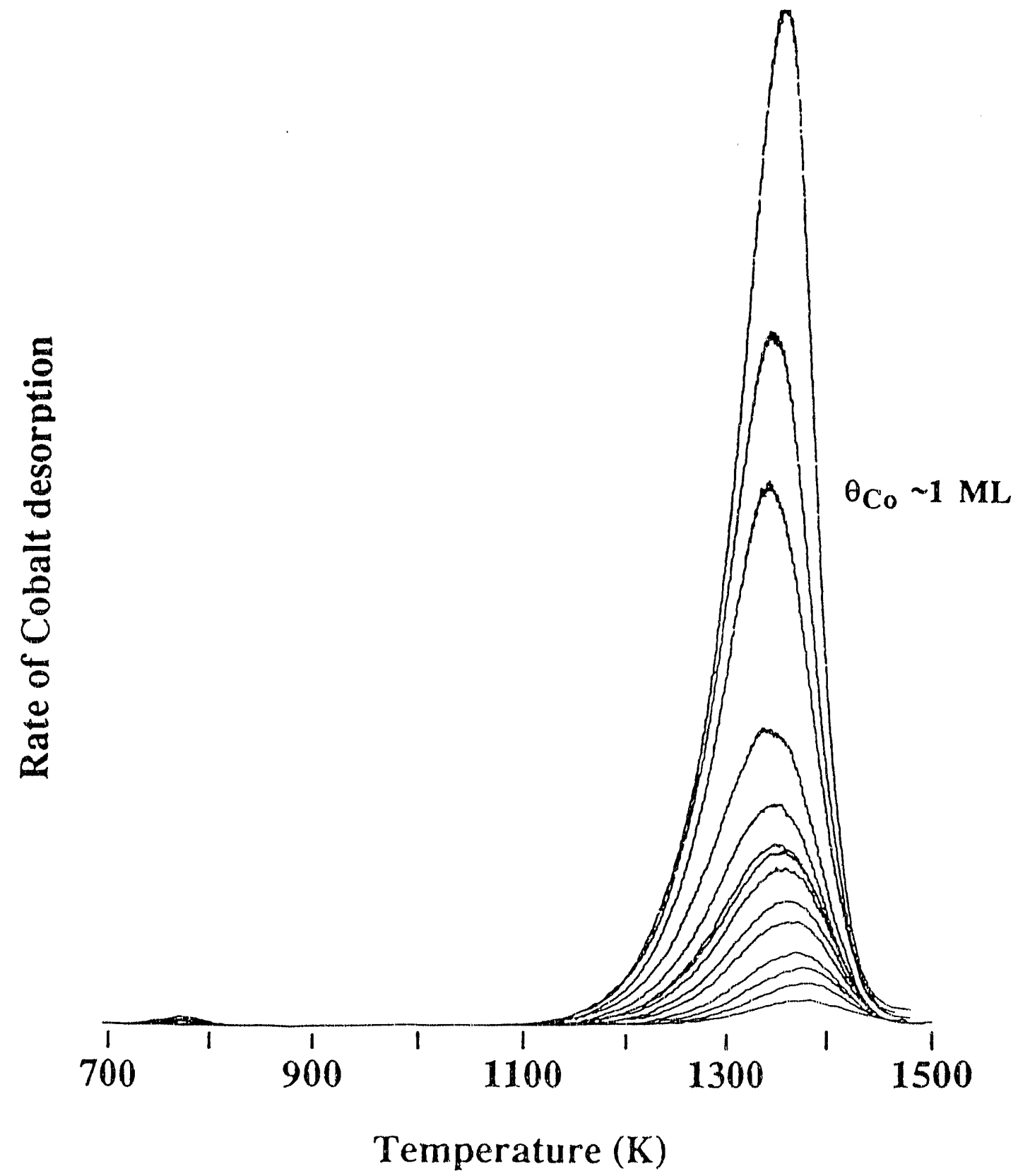

Figure 4.13: Cobalt desorption from $\operatorname{Mo}(28,4,1)-c(2 \times 2) S$ surfaces. 
$\mathrm{T}_{\mathrm{p}}$ with increasing coverage. As the cobalt coverage increases, sufficiently large two dimensional islands are formed on the terraces and the desorption becomes similar to that observed from the flat $(100)$ surface.

Preadsorbed sulfur both leads to a doubling of the terrace width ${ }^{22}$ (section 5.4) and helps in ordering low coverages of cobalt into two dimensional islands. Therefore in the presence of sulfur, first order kinetics are observed. In chapter 5, low energy electron diffraction results are presented which support these claims that sulfur restructures the molybdenum surface and aids in the ordering of cobalt overlayers. Depositing cobalt on a crystal held at $473 \mathrm{~K}$ or above, allows the cobalt overlayer to initially form two dimensional islands. Cobalt then desorbs from these islands with first order kinetics. This shows that the initial adsorption configuration is different depending on whether cobalt is adsorbed on molybdenum surfaces held at room temperature or $473 \mathrm{~K}$ and above. The initial adsorption configuration is stable up to the desorption temperature.

\subsection{Summary}

In this chapter, we have investigated the morphology of cobalt overlayers on molybdenum surfaces. While Auger spectroscopy and $\mathrm{CO}$ chemisorption studies suggest a Frank-Van der Merwe growth mode for cobalt overlayers on both flat and vicinal surfaces, cobalt desorption studies indicate differences between the initial configuration of the cobalt overlayer on flat and vicinal surfaces. Cobalt submonolayers desorb with first order kinetics from the Mo(100) surface. On the vicinal surfaces, below 5 / $8 \mathrm{ML}$, cobalt desorption cannot be simply described by first order kinetics with a constant activation energy of desorption and prefactor. At low cobalt coverages, the temperature of cobalt desorption is significantly higher on the $\operatorname{Mo}(911)$ and $M o(28,4,1)$ surfaces than on the 
Mo(100) surface. In light of the results that will be presented in chapter 5 , these differences might be explained by cobalt decoration of the molybdenum step edges. When cobalt is chemisorbed onto sulfur covered molybdenum surfaces, sulfur migration on top of the cobalt overlayer is seen. While this topmost sulfur overlayer has only a negligible effect on cobalt desorption from $\mathrm{Mo}(100)$ surfaces, it substantially changes cobalt desorption from vicinal surfaces. In the presence of sulfur, cobalt exhibits first order desorption kinetics in the submonolayer regime. For cobalt coverages less than $5 / 8 \mathrm{ML}$, the temperature of cobalt desorption is lower on the sulfur covered $\mathrm{Mo}(911)$ and $\operatorname{Mo}(28,4,1)$ surfaces than on the clean $M o(911)$ and $\operatorname{Mo}(28,4,1)$ surfaces.

\section{References}

[1] A. Zangwill, Physics at Surfaces, Cambridge university press, Cambridge, New York, Melbourne, (1988).

[2] E. Bauer, Appl. Surf. Sci. $11 / 12479$ (1982).

[3] W. R. Tyson and W. A. Miller, Surf. Sci. 62267 (1977).

[4] F. C. Frank and J. H. Van der Merwe, Proc. R. Soc. London Ser. A 198205 (1949); W. A. Jesser and D. Kuhlmann-Wilsdor, Phys. Status Solidi 1995 (1965); J. H. Van der Merwe and C. A. Bell in Epitaxial Growth edited by J. Matthew's.

[5] J. W. Matthews, J. Vac. Sci. Technol. 12126 (1974).

[6] M. H. Yang and C. P. Flynn, Phys. Rev. Lett. 622746 (1989).

[7] A. M. de Jong and J. W. Niemantsverdriet, Vacuum 41232 (1990).

[8] P. J. Berlowitz and D. W. Goodman, Surf. Sci. 187463 (1987).

[9] T. E. Felter and P. J. Estrup, Surf. Sci. 76464 (1978). 
[10] H. Niehus, Surf. Sci. 9288 (1988).

[11] E. I. Ko and R. J. Madix, Surf. Sci. 100 L 505 (1980).

[12] F. Zaera, E. B. Kollin and J. L. Gland, Chem Phys. Lett. 121464 (1985).

[13] H. Papp, Surf. Sci. 149460 (1985).

[14] U. Bardi, P. Tiscione and G. Rovida, Appl. Surf. Sci. 27299 (1980).

[15] M. E. Bridge, C. M. Comrie and R. M. Lambert, Surf. Sci. 67393 (1977).

[16] M. E. Bussell, Ph. D. thesis, University of California, Berkeley (1988).

[17] Handbook of Chemistry and Physics, 68 th edition, CRC Press Inc. (1987).

[18] W. F. Egelhoff and D. A, Steigerwald, J. Vac. Sci. Technol. A7 2167 (1989).

[19] L. J. Clarke, Surf. Sci. 102331 (1981).

[20] J.-W. He and D. W. Goodman, Surf. Sci. 24529 (1991).

[21] JANAF Thermochemical tables, J. Phys. Chem. Ref. Data, Vol 14, Suppl. 1 (1985).

[22] C. C. Knight and G. A. Somorjai, accepted to Surf. Sci. (1992). 


\section{Chapter 5 Structure of Adsorbate Overlayers on Flat and Vicinal Mo(100) Surfaces}

\section{$5.1 \quad$ Introduction}

In chapter 3 , the structure of three clean, vicinal $M o(100)$ surfaces was characterized and in chapter 4, the growth of adsorbates on the flat and vicinal $\operatorname{Mo}(100)$ surfaces was presented. Now, in this chapter we discuss the structure of adsorbate overlayers and adsorbate induced restructuring of vicinal molybdenum surfaces. The chapter is organized in the following manner. Following this introduction, some theoretical considerations on diffraction from overlayers on stepped surfaces are summarized. The next two sections compare the structure of sulfur and cobalt overlayers on flat and vicinal $\mathrm{Mo}(100)$ surfaces. In section 5.5, the structure of cobalt overlayers on sulfur covered molybdenum surfaces is explored. Finally, in section 5.6, an overview is given of the results contained in this chapter.

While there are many detailed experimental and theoretical studies of phase diagrams of oxygen, sulfur, hydrogen, alkali metals and other chemisorbed species on a variety of low Miller index surfaces, there is only a limited number of comparable experimental and theoretical studies of the phase diagram of adsorbates on stepped surfaces. This is unfortunate since these studies should be very interesting and could potentially lead to an increased knowledge about the adsorption of atoms and molecules on solid surfaces. These studies would facilitate a comparison between the number of 
adsorbed phases on flat and vicinal surfaces as well as determine if even different phases exist on flat and vicinal surfaces.

Experimental results on adsorbates on stepped surfaces include the work of Lanzillotto and Bernasek ${ }^{1}$ who investigated the behavior of sulfur on $\mathrm{Pt}_{\mathrm{t}}(\mathrm{S})-[6(111) \times 100]$ using low energy electron diffraction (LEED). They found that sulfur orders in a $p(2 \times 2)$ overlayer at the step edges ar coverages between $0.1-0.15 \mathrm{ML}$. These are lower coverages than that necessary to form the $\mathrm{p}(2 \times 2)$ overlayer on the flat (111) surface. Sokolowski and Pfnür have studied the $\mathrm{p}(2 \times 2) \mathrm{O}^{2}$ and $\mathrm{p}(2 \times 2) \mathrm{S}^{3}$ overlayers formed on a stepped $\mathrm{Ru}(0001)$ surface at coverages of $0.25 \mathrm{ML}$ also using LEED. Despite the similarity of these structures, significant differences exist in the behavior of the fractional order beams. For the oxygen covered surface, both integer and half order beams appear as doublets of identical width at characteristic energies. This result indicates the growth of large $\mathrm{p}(2 \times 2)$ domains and thus shows that long range adsorbate-adsorbate correlations exist between different terraces. By contrast, on the sulfur covered surface the fractional beams are broad, but never split. In this case the growth of sulfur on different terraces is uncorrelated. Schematic representations of a generic $\mathrm{p}(2 \times 2)$ overlayer with and without adsorbate correlations between adjacent terraces are shown in figure 5.1. These examples show the complexity of the phenomena involved in adsorbate ordering on stepped surfaces.

Steps might also have an influence on the growth of overlayer domains. On a flat surface there is usually a statistical distribution of different overlayer domains. Different domains are separated by domain walls and the average domain width is evident from the size of the overlayer diffraction spots. Steps can modify the growth of the overlayer, leading to the formation of a single large domain. Not only can steps influence overlayer growth, but the adsorbate can modify the step structure, ie. restructure the surface. This phenomenon of adsorbate induced restructuring of stepped surfaces has recently received considerable attention. 
(a)

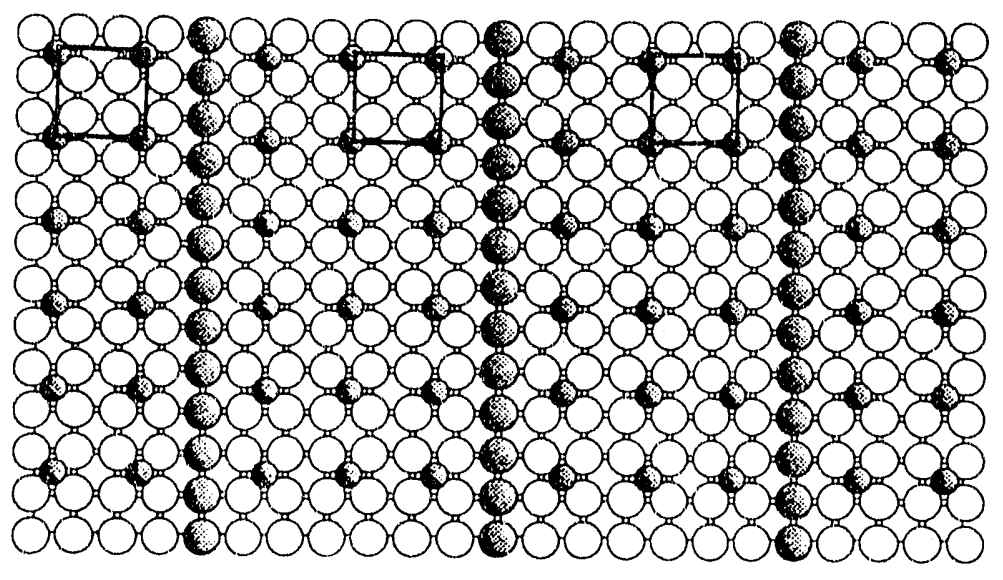

(b)

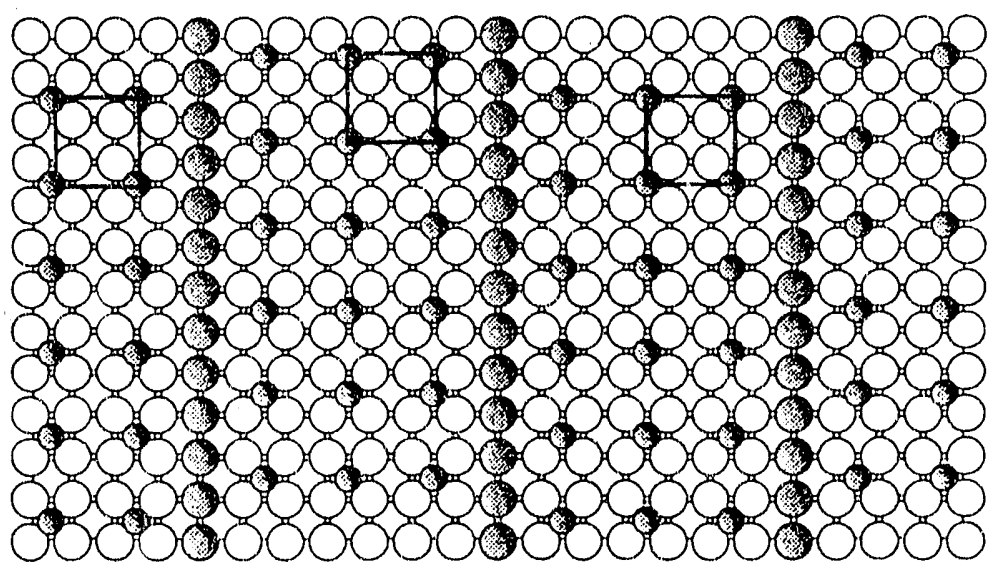

(c)

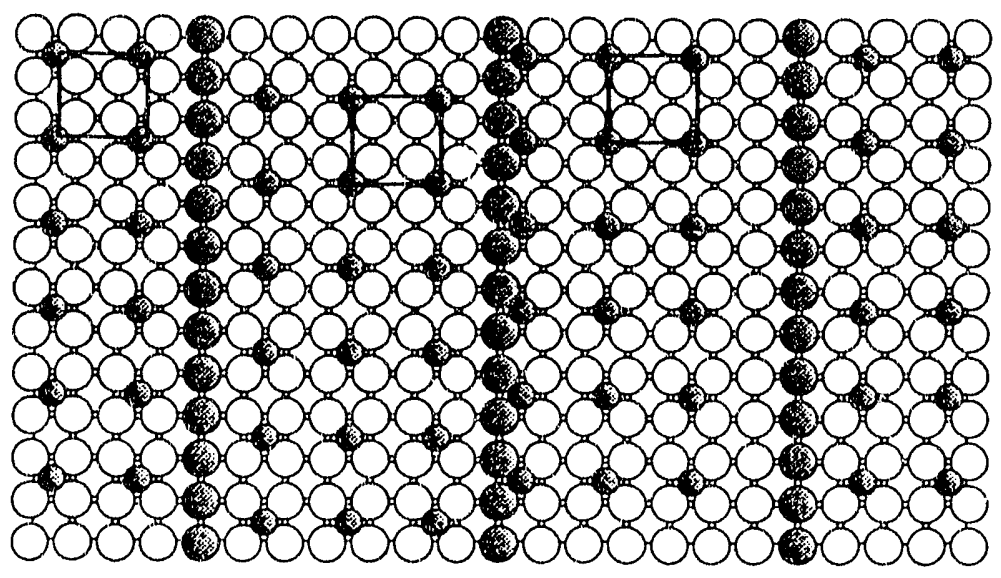

Figure 5.1: A ( $2 \times 2)$ overlayer on a square unit cell showing (a) correlations both perpendicular and parallel to the step edges and (b) only perpendicular correlations. In (c) there are no adsorbate correlations between adjacent terraces. 
Lanzillotto and Bernasek ${ }^{1}$ and Comsa et al. ${ }^{4}$ have shown that both sulfur and oxygen lead to a doubling of the step height on vicinal Pt(111) surfaces. On the other hand, Dowben et al. ${ }^{5}$ have reported that dissociatively adsorbed nitrogen induces reconstruction that leads to an increase in the step density on the $\mathrm{Fe}(\mathrm{S})-[11(100) \times 2(110)]$ surface. Their results indicate that with the smallest surface concentration of nitrogen, terraces six to seven atoms wide and monoatomic height steps are formed, indicating that nitrogen pins the steps. At higher nitrogen coverages, a $c(2 \times 2)$ structure attributed to the surface nitride $\mathrm{Fe}_{4} \mathrm{~N}$ is seen. The authors suggested that by decreasing the step height and the terrace width, the strain caused by the lattice mismatch between the nitride overlayer and (100) terraces of the substrate is reduced.

\subsection{Diffraction from Overlayers on Vicinal Surfaces}

It should be clear from the introduction, that a discussion of diffraction from overlayers on stepped surfaces involves many considerations. These include the influence of steps on ordering of adsorbates, adsorbate induced restructuring of stepped surfaces, the overlayers that grow on the stepped surface and the possibility of preferential nucleation of a given domain. The above effects are easy to determine experimentally. In chapter 3 , it was shown that the average terrace width can be derived from the size of spot splitting while the step height can be determined from the energy dependence of the spot sharpness. If the adsorbate restructures the surface and leads to a new terrace width and step height, this fact can be directly determined from the size of spot splitting of the substrate spots and the energy dependence of the sharpness of the substrate spots. Analysis of the substrate spots alone is thus sufficient to determine if the adsorbate restructures the stepped surface. 
The fractional order beams, on the other hand, show which overlayer structures evolve on the stepped surface. If the terraces on a stepped surface are sufficiently wide, structures similar to those ordered on the nominally flat surface might nucleate on the terraces. However, the step orientation could prevent the ordering of certain overlayer structures. This can occur when nucleation occurs at the step edges, rather than randomly on the terraces, and the overlayer unit vectors run in directions very different from the step direction.

Steps can also be domain selective. In general whenever there is a surface structure which has lower symmetry than the substrate symmetry, different rotational domains of the structure will be present on the surface. For adsorbates which have ordered structures with unit cells larger than the unit cell of the substrate, there is also the possibility to form translational domains. Figure 5.2 shows an example of both types of domains and the corresponding domain walls. The different domains have the same internal atomic configurations. They are thus energetically identical and are present with equal distribution on low Miller index surfaces. If the domains are larger than the coherence length of the electron beam, the different domains will contribute independently to the diffraction pattern. The observed diffraction pattern will be a sum of the individual diffraction patterns that would be seen for the separate domains. The presence of steps reduces the symmetry compared with the corresponding low Miller index surface. Because steps introduce this anisotropy, one particular domain orientation might now be energetically favored on the surface. The presence of domain areas smaller than the coherence length of the electron beam results in the weakening and broadening of fractional order beans and an increased background intensity.

For a clean, ordered stepped surface, there is positional correlation between atomis on different terraces. Therefore there is a well defined vector (figure 3.1) connecting identical lattice sites on adjacent terraces. For adsorbate covered surfaces, nucleation can 
(a)

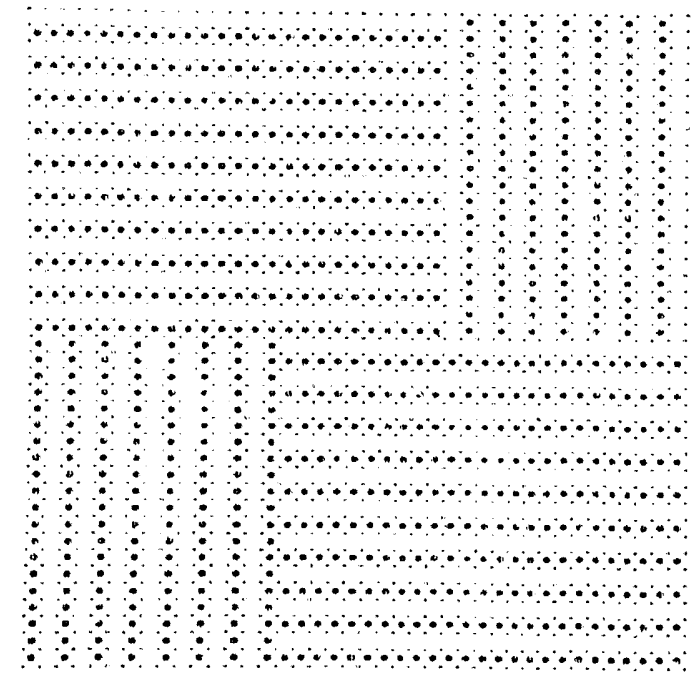

(b)
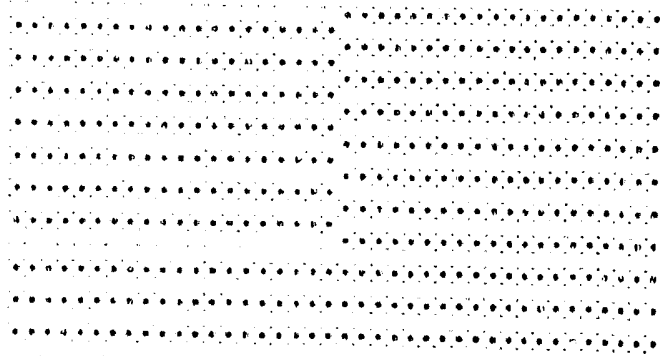

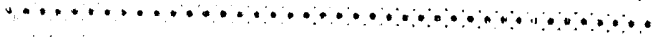

$\ldots \cdots \cdots \cdots \cdots \cdots \cdots \cdots \cdots \cdots \cdots$
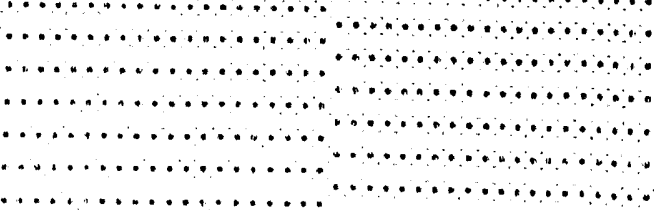

Figure 5.2: A (2×1) superlattice (large dots) on a square lattice (small dots) showing different (a) rotational domains and (b) translational domains. 
occur randomly on terrace sites rather than at step edges. If there is random nucleation, then different rotational domains are randomly formed on adjacent terraces. Alternatively, there can be random translational displacements both parallel and perpendicular to the step edges. In either case, there is no well defined vector connecting identical adsorbate sites on different terraces. Figure 5.1 (c) showed an example for such uncorrelated nucleation. Random translational displacements parallel to the step edges can occur for some overlayer structures even when nucleadion occurs at the step edges. Nucleation at step edges only insures that there are no random translational displacements perpendicular to the step edges if the system has a well defined terrace width. This situation is schematically shown in figure 5.2 (b). Calculations in the kinematic approximation show that interference between non-correlated islands on different terraces has only a negligible effect on the shape of diffraction beamis ${ }^{6}$. As a consequence, no oscillation of overlayer beams between singlets and doublets as a function of electron energy is observed. In general, the overlayer beams will be broadened by the finite size of the overlayer islands.

On the other hand, there are examples such as $\mathrm{O} /$ stepped $\mathrm{Ru}(0001)^{2}$ where there are no random rotational or translational displacements between islands on different terraces. Such a case is shown in figure 5.2 (a). In this case, there are clear positional correlations between overlayer structures on different terraces. It is not understood why such long range correlations exist for particular overlayers. One idea is that these correlations are adsorbate mediated. If the adsorbate nucleates at the step edges and restructures the step edges, this reconstruction can dictate where atoms reside on neighboring terraces. This can thus force positional correlations between overlayer structures on adjacent terraces. Alternatively, the width of the terrace can force adsorbate correlations normal, but not parallel, to the step edges (see figure 5.2 (b)). If a structure grows additionaliy with unit periodicity parallel to the step edges, positional correlations on neighboring terraces will necessarily exist. 
For positionally correlated overlayers, there is a well defined vector connecting identical adsorbate sites on different terraces. Therefore there will be strong constructive and destructive interference from electrons scattered from overlayer atoms on different terraces. Oscillation between doublets and singlets will be seen for both overlayer beams and substrate beams as the electron energy is varied.

In summary, the size of splitting of the substrate spots (or overlayer spots when splitting occurs) characterizes the average terrace width while the step height can be determined from the energy dependence of the sharpness of the substrate (or overlayer) beams. The identification and possible splitting of the overlayer beams shows which overlayers grow on the stepped surface and if the domains of adsorbate atoms on different terraces have positional correlations.

\subsection{Sulfur Overlayers on Molybdenum Surfaces}

Sulfur overlayers on the (100) surface of molybdenum form different structures depending on the coverage and annealing temperature 7,8 . In this work $c(2 \times 2),\left|\begin{array}{ll}2 & 1 \\ 1 & 1\end{array}\right|$, $c(4 \times 2)$ and $p(2 \times 1)$ sulfur structures were seen on the $M o(100)$ substrate. The preparation of these overlayers was described in section 2.2.4. Even though these structures ideally correspond to coverages of one-half, two-thirds, three-quarters and one monolayer, they are observed for ranges between $0.2-0.5,0.6-0.7,0.75-0.9$ and $0.95-1.05 \mathrm{ML}$ respectively. In between these ranges, diffraction spots from two structures are visible. This indicates that at these coverages, the surface is covered with large domains of different, coexisting structures. As the sulfur coverage is increased, the intensity of the diffraction spots from the higher coverage structure increases. This is accompanied by a continuous decrease in the intensity due to the domain with lower sulfur 


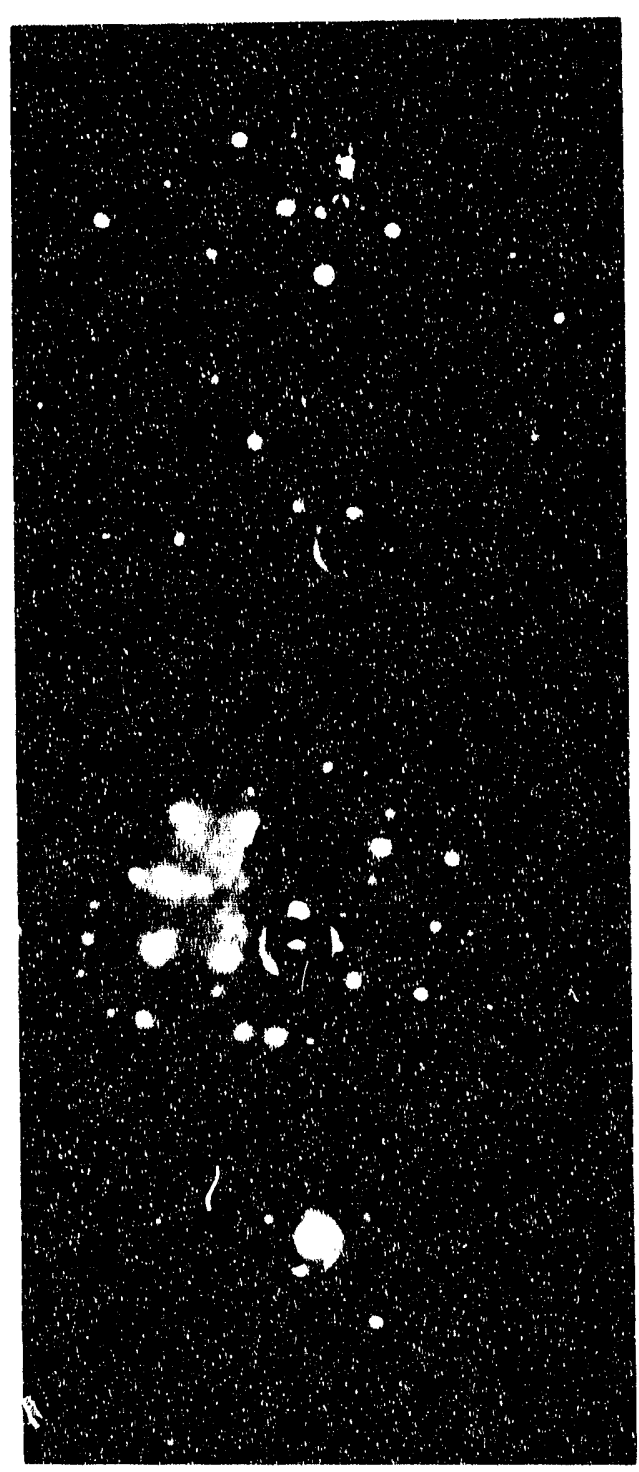

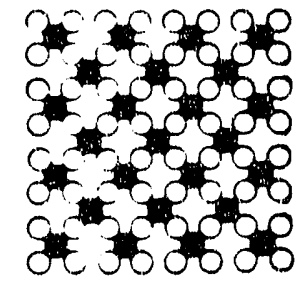
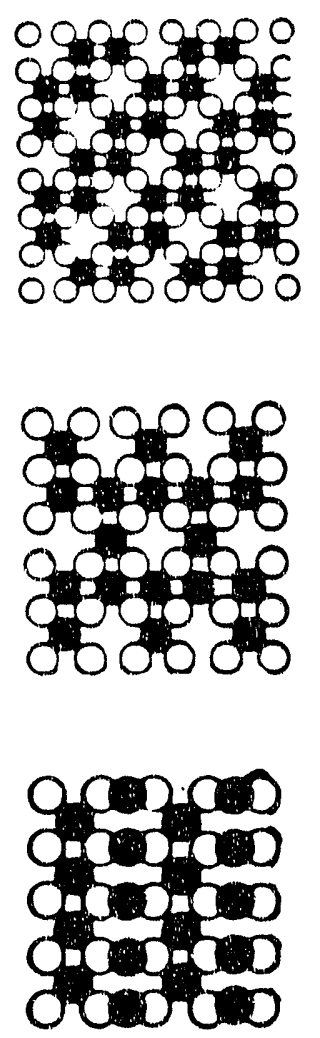

\author{
$140 \mathrm{eV}$ \\ $c(2 \times 2)$ \\ $(-)_{S}=0.5 \mathrm{ML}$
}

$89 \mathrm{eV}$
21
11
$\Theta_{S}=0.67 \mathrm{ML}$

$120 \mathrm{eV}$

$c(4 \times 2)$
$\Theta_{S}=0.75 \mathrm{ML}$

$115 \mathrm{eV}$

$p(2 \times 1)$

$\Theta_{S}=1 \mathrm{ML}$

XBB 910-8013A

Figure 5.3: Diffraction patterns and proposed real space models for $\mathrm{c}(2 \times 2)$,

$\left|\begin{array}{ll}2 & i \\ 1 & 1\end{array}\right|, c(4 \times 2)$ and $p(2 \times 1)$ sulfur overlayers on $M o(100)$ surfaces. 
coverage. Proposed models for these structures and observed diffraction patterns are shown in figure 5.3.

Only the lowest coverage sulfur structure has been solved by dynamical low energy electron diffraction analysis. There is general agreement that for the $c(2 \times 2)$ structure ${ }^{9,10.11}$, sulfur is adsorbed in highly symmetric fourfold sites on the $\mathrm{Mo}(100)$ surface. A scanning tunneling microscopy study of the high coverage (one monolayer) $\mathrm{p}(2 \times 1) \mathrm{S}$ structure suggested that sulfur was adsorbed in asymmetric sites halfway between fourfold and bridge sites ${ }^{12}$. However this structure possesses a glide plane and would result in extinction of the $(0,(n+1) / 2)$ beams when the diffraction pattem is viewed at normal incidence. Such extinctions have never been observed in this work nor have they been reported in any other work. Work in progress in this group is aimed at determining the atomic configurations of this structure using dynamical diffraction analysis ${ }^{13}$. The scanning tunneling microscopy results are also in contradiction with total energy calculations. These computations favor a $\mathrm{p}(2 \times 1)$ structure in which sulfur atoms are adsorbed in symmetric bridge and fourfold sites ${ }^{14}$. The two different structural models proposed for the $M o(100)-p(2 \times 1) S$ surface are shown in figure 5.4. More recent tunneling experiments in the same group suggest that a well ordered $\mathrm{p}(2 \times 1) \mathrm{S}$ structure does not exist on the Mo(100) surface ${ }^{15}$. This is based on their observation that diffraction patterns of their $\mathrm{Mo}(100)-\mathrm{p}(2 \times 1) \mathrm{S}$ surfaces had increased background intensity in quarter order positions from domains of $c(4 \times 2)$ structures. Additionally their STM images of the $M o(100)$ - $p(2 \times 1) S$ and $M o(100)-c(4 \times 2) S$ surfaces indicated that these surfaces had the same unit cell. Since atomic resolution of sulfur atoms was not achieved for these images, this claim awaits further unambiguous experimental support.

On both the stcpped $(910)$ and kinked $(28,4,1)$ surfaces, only the $c(2 \times 2)$ and $p(2 \times 1)$ structures are observed, while ordered $\left|\begin{array}{ll}2 & 1 \\ 1 & 1\end{array}\right|$ and $c(4 \times 2)$ structures can not be detected ${ }^{16}$. On the stepped (911) surface, only the $\mathrm{c}(2 \times 2)$ structure is formed. 


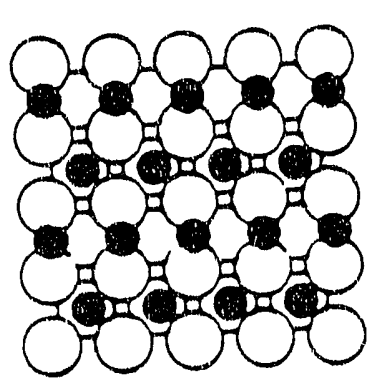

(a)

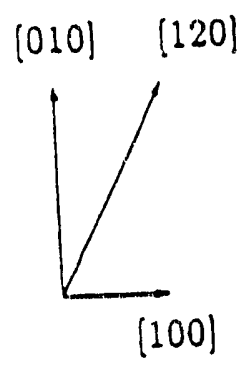

$(100)$

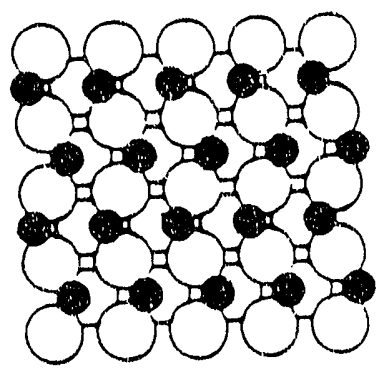

(b)

Figure 5.4: Models of Mo(100) - $\mathrm{p}(2 \times 1) \mathrm{S}$ surface showing the (a) bridge-hollow and (b) asymmetric site models. 
Additionally, on the vicinal surfaces, $\mathrm{c}(2 \times 2)$ diffraction spots are seen at lower coverages (below $0.1 \mathrm{ML}$ ) than on the flat $\mathrm{Mo}(100)$ surface. This indicates that at these low coverages, sulfur atoms coalesce and order along step edges. This implicates step edges as important sites for sulfur nucleation and ordering on the vicinal surfaces.

The main difference between the diffraction pattern of the $c(2 \times 2)$ structures on the flat and vicinal surfaces is that for the latter the integer order substrate spots were observed to oscillate between singlets and doublets as the primary energy was changed. The energies at which the integer order ( $0 \overline{1})$ spot appeared as singlets and doublets are given in table 5.1. The numbers reported are those for the kinked $(28,4,1)$ surface. However they are similar within experimental error $(< \pm 5 \mathrm{eV})$ to the values obtained on the other vicinal surfaces. Typical diffraction patterns of the $c(2 \times 2)$ structure on the stepped (910) and kinked $(28,4,1)$ surfaces are shown in figure 5.5. Directly from the diffraction pattern it can be seen that the spot splitting distance is about one half of the size on the clean surface (compare with figure 3.3). This indicates the presence of terraces that are twice as wide as on the clean surface. On the sulfur covered surfaces, the average terrace widths are nine, nine, and seven atoms on the (910), (911) and $(28,4,1)$ surfaces respectively. Double height steps are present on these sulfur covered surfaces. This is confirmed by the energies at which spot splitting occurs.

Splitting of half order diffraction beams from the Mo(910) $-c(2 \times 2) S$ and Mo $(28,4,1)-c(2 \times 2) S$ surfaces was never observed. This finding suggests uncorrelated adsorbate ordering between neighboring terraces. Even though both sulfur nucleation and the growth of the $c(2 \times 2)$ overlayer seems to originate at the step edges, there exists no adsorbate-mediated mechanism or other mechanism to force overlayers on different terraces to have positional correlations parallel to the step edge. Therefore there are random translational displacements between $\mathrm{c}(2 \times 2) S$ islands on different terraces. A model of the proposed surface is shown in figure 5.6 (b). On the other hand, the half order beams from 
Table 5.1: Experimental energies at which singlets and doublets are seen for the $(0 \overline{1})$ and equivalent beams for sulfur covered surfaces. The data given was obtained for the $\operatorname{Mo}(28,4,1)$ surface. The calculated energies listed for comparison were obtained by assuming that two atom height steps were present.

\begin{tabular}{|c|c|c|c|c|c|c|}
\hline \multirow[b]{3}{*}{$s$} & \multirow{2}{*}{\multicolumn{2}{|c|}{ Calculated }} & \multicolumn{4}{|c|}{ Experimental } \\
\hline & & & \multicolumn{2}{|c|}{$c(2 \times 2) S$} & \multicolumn{2}{|c|}{$(1 \times 1) S$} \\
\hline & singlets & doublets & singlets & doublets & singlets & doublets \\
\hline 1.5 & & 18 & & & & \\
\hline 2 & 24 & & & & & \\
\hline 2.5 & & 32 & & 30 & & \\
\hline 3 & 42 & & 45 & & 42 & \\
\hline 3.5 & & 54 & & 60 & & 58 \\
\hline 4 & 69 & & 72 & & 72 & \\
\hline 4.5 & & 85 & & 96 & & 86 \\
\hline 5 & 105 & & 125 & & 115 & \\
\hline 5.5 & & 123 & & 135 & & 125 \\
\hline 6 & 146 & & 153 & & 150 & \\
\hline 6.5 & & 168 & & & & 160 \\
\hline 7 & 193 & & 180 & & 200 & \\
\hline 7.5 & & 221 & & 220 & & 220 \\
\hline 8 & 251 & & & & 250 & \\
\hline
\end{tabular}


(a)

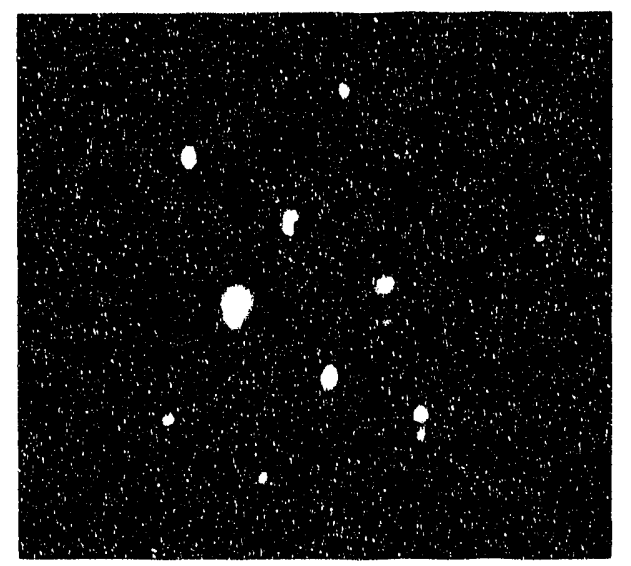

(b)

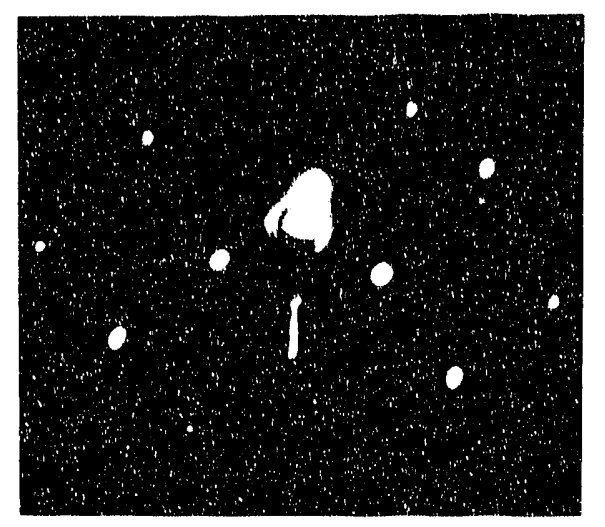

XBB $923-1803$

Figure 5.5: Diffraction patterns of $c(2 \times 2) S$ overlayers on (a) $\mathrm{Mo}(28,4,1), 135 \mathrm{eV}$ and (b) Mo(910), $83 \mathrm{eV}$. 
(a)

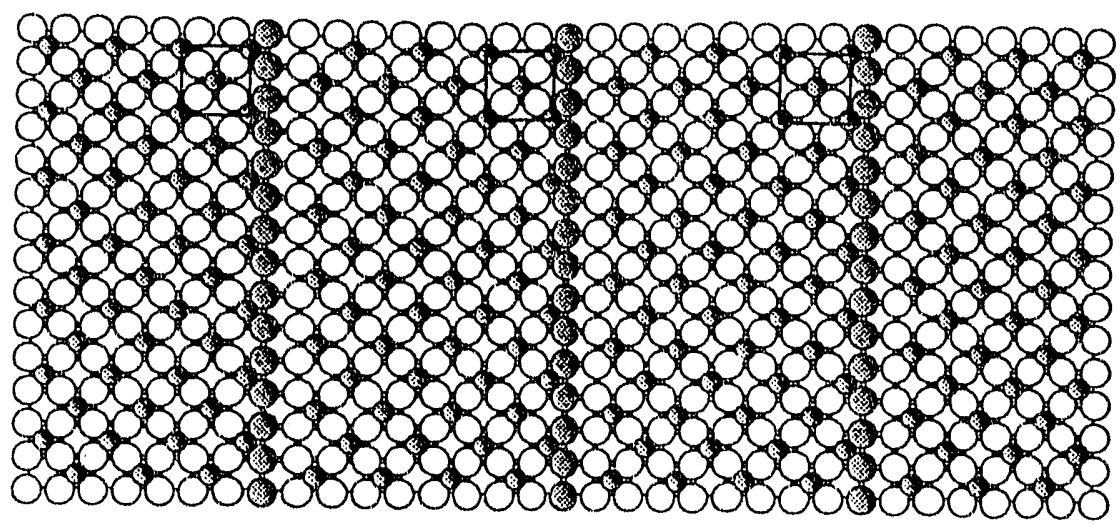

(b)

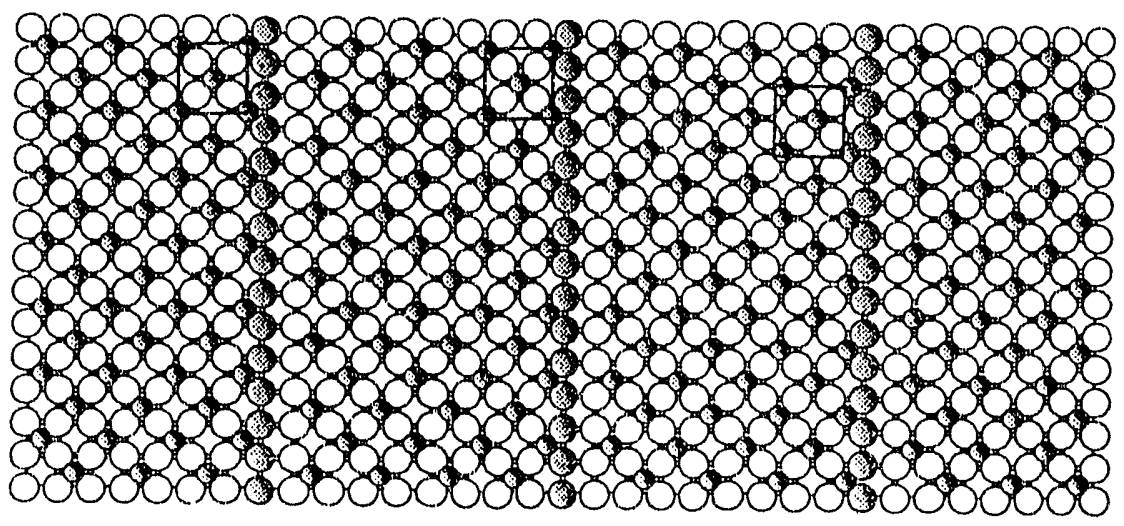

(c)

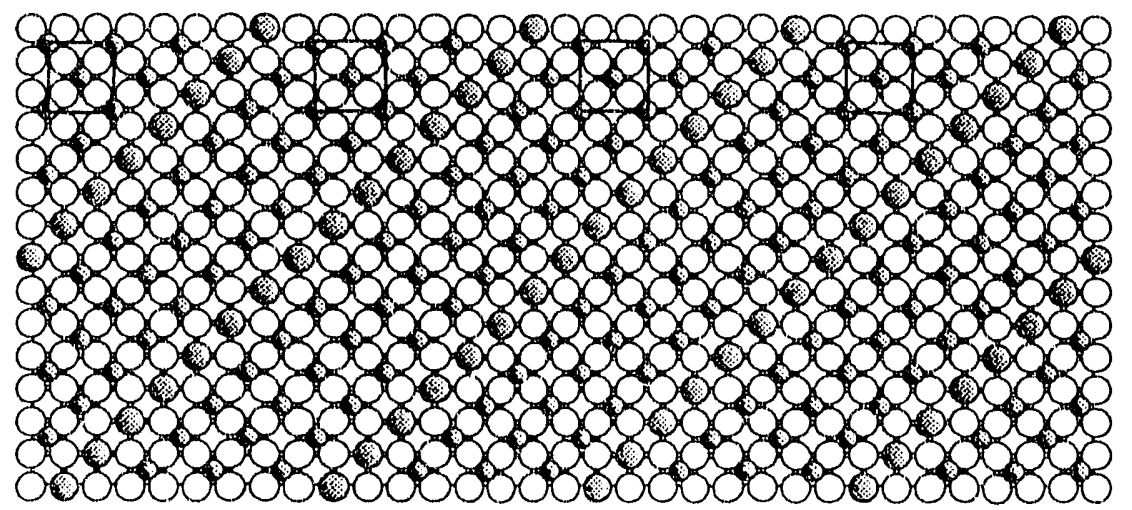

Figure 5.6: Real space models of (a) the $M o(910)-c(2 \times 2) S$ surface showing correlated sulfur ordering between neighboring terraces, (b) the Mo(910) - c(2 × 2)S surface showing uncorrelated sulfur ordering between neighboring terraces ((b) appears to be the correct model) and, (c) the $\operatorname{Mo}(911)-c(2 \times 2) S$ surface showing that the step direction forces correlations between sulfur atoms on different terraces. 
the Mo(911)-c(2 X 2)S surface were split at characteristic energies. A model for this surface is shown in figure 5.0 (c). While adsorbate correlation perpendicular to the step edges is facilitated by sulfur nucleation at these positions, correlation parallel to the step edges is accommodated by the step direction. On this surface, because of the step direction, all of the metal step edges are necessarily decorated with sulfur atoms. This result shows that step directions can in some cases force positional correlations between adsorbate islands on different terraces.

The presence of steps result in preferential orientation of one domain. A comparison of the diffraction patterns of the $\mathrm{p}(2 \times 1) \mathrm{S}$ structures on the flat and vicinal $\mathrm{Mo}(100)$ surfaces indicate that on the vicinal surfaces, one domain is much better ordered (figure 5.7). For the well ordered domain, the diffraction spots are as sharp as the sharpest integer order spots. This indicates the presence of rather large domains, about $100 \AA$ or more. Diffuse streaking is observed in the regions of $\mathrm{k}$ space where diffraction spots from the second domain would be expected, indicating that these domains are definitely much smaller than $100 \AA$. The preferentially ordered domains correspond to those with the longer side of the unit cell perpendicular to the step edges. Within these domains, sulfur atoms can be located on all of those metal atoms which are at the step edges. Preferential ordering of these domains maximizes the number of sulfur-step atom bonds, suggesting a strong interaction between sulfur atoms and metal step edges. This stronger interaction determines which ordered domains grow on the terraces.

On the flat (100) surface, well ordered sulfur structures are observed at coverages of approximately two-thirds and three-quarters of a monolayer. However, despite numerous attempts which included varying the sulfur coverage, annealing temperature and time, no well ordered $c(4 \times 2)$ or $\left|\begin{array}{ll}2 & i \\ 1 & 1\end{array}\right|$ overlayers were seen on these vicinal surfaces in this coverage range. In some experiments, increased diffuse intensities in the quarter order positions expected for the $c(4 \times 2)$ structure was seen. However well defined diffraction 
(a)

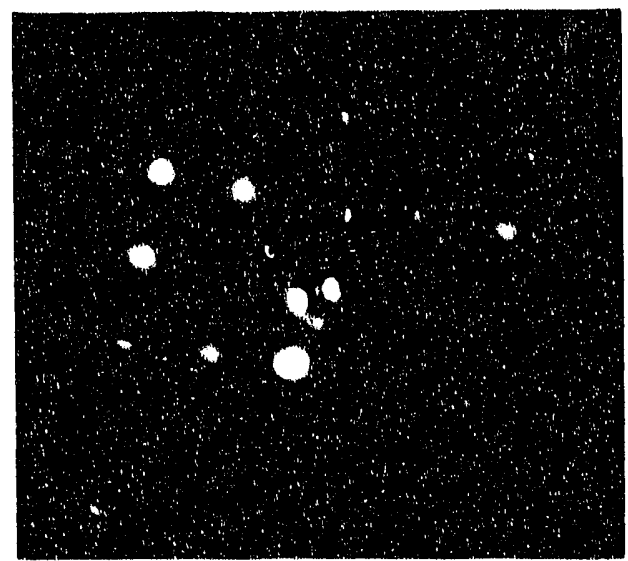

$122 \mathrm{eV}$

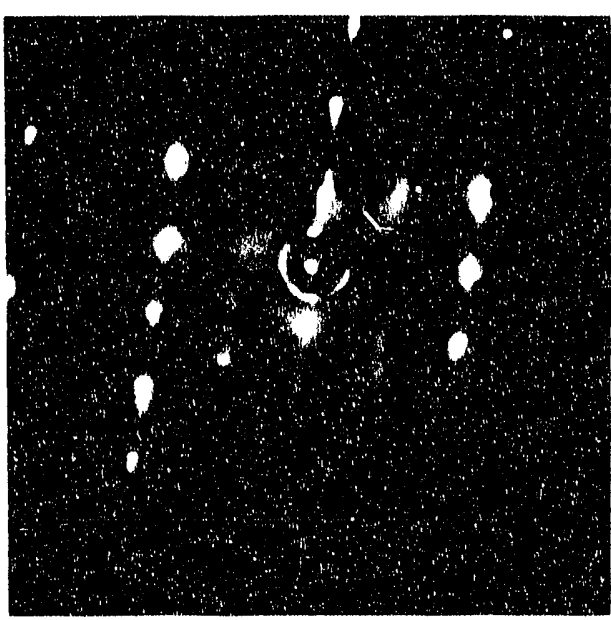

$125 \mathrm{eV}$

(b)

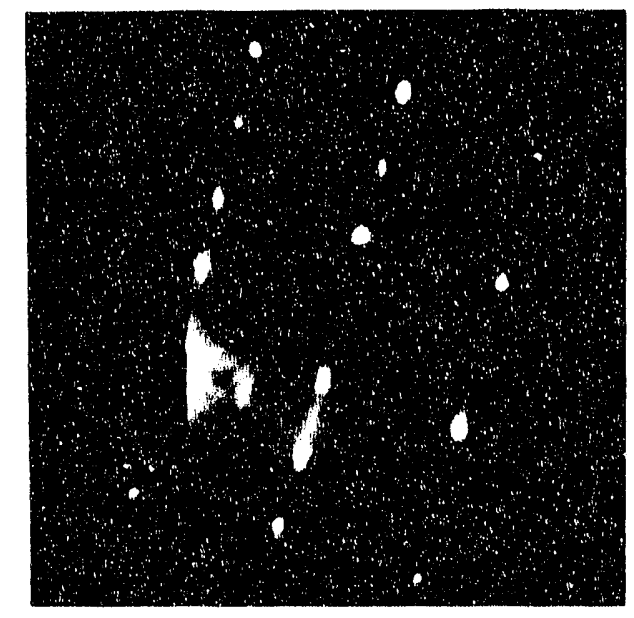

$130 \mathrm{eV}$

(c)

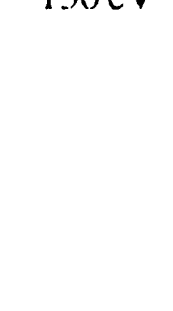

XBB $910-9438 A$

Figure 5.7 Diffaction patterns of $\mathrm{p}(2 \times 1) \mathrm{S}$ overlayer on (a) $\operatorname{Mo}(100)$, (b) Mo(910) and (c) $\operatorname{Mo}(2 \times, 4,1)$ surfiaces. 
beams were never observed. There was no indication at all of $\left|\begin{array}{ll}2 & 1 \\ 1 & 1\end{array}\right|$ spots. It is not possible to arrange either the $\left|\begin{array}{ll}2 & i \\ 1 & 1\end{array}\right|$ or $\mathrm{c}(4 \times 2)$ overlayers on the terraces, and still decorate all of the metal step atoms with adsorbate atoms (see figures 5.3 (b) and (c)). So instead at sulfur coverages between one-half and three-quarters of a monolayer, a poorly ordered (1X 1) diffraction pattern was observed on the vicinal surfaces. The ordering of sulfur atoms along the step edges thus determines which ordered structures are formed on the terraces and which urdered domain grows.

A diffraction pattern of the $M o(28,4,1)-(1 \times 1)$ S surface which shows the split spots is reproduced in figure 5.8. The energies at which integer spots appeared as singlets and doublets for the $c(2 \times 2) S$ and $(1 \times 1) S$ structures on the kinked $(28,4,1)$ surface were given in table 5.1. The agreement in the frequency of oscillations between singlets and doublets between the experimental values and those calculated for double height steps is evidence that the adsorption of sulfur induces the formation of diatomic steps. Observation of the diffraction pattern of the (1X 1) sulfur covered kinked Mo $(28,4,1)$ surface shows that $\Delta \mathrm{K}_{01} / \mathrm{K}_{01}$ is reduced to $1 / 7$. This spot splitting distance is consistent with the presence of diatomic steps separating terraces that are on the average composed of seven molybdenum atoms ${ }^{17}$. This sulfur induced step-doubling is completely reversible on all of the surfaces. Heating the crystal above $1900 \mathrm{~K}$ to desorb the sulfur restores the monoatomic steps characteristic of the clean surfaces. Table 5.2 summarizes the sulfur superstructures formed on the different molybdenum surfaces.

That sulfur induces step-doubling on molybdenum surfaces is corroborated by scanning tunneling microscopy images of sulfur overlayers on $\mathrm{Mo}(100)^{15}$. Nominally flat Mo(100) crystals have large terraces separated predominantly by monoatomic steps of random orientation. After sulfur is adsorbed on a $\mathrm{Mo}(100)$ crystal, predominantly diatomic steps are observed. These tunneling studies show that sulfur induced step-doubling accurs irrespective of the terrace width or step orientation on molybdenum surfaces. 


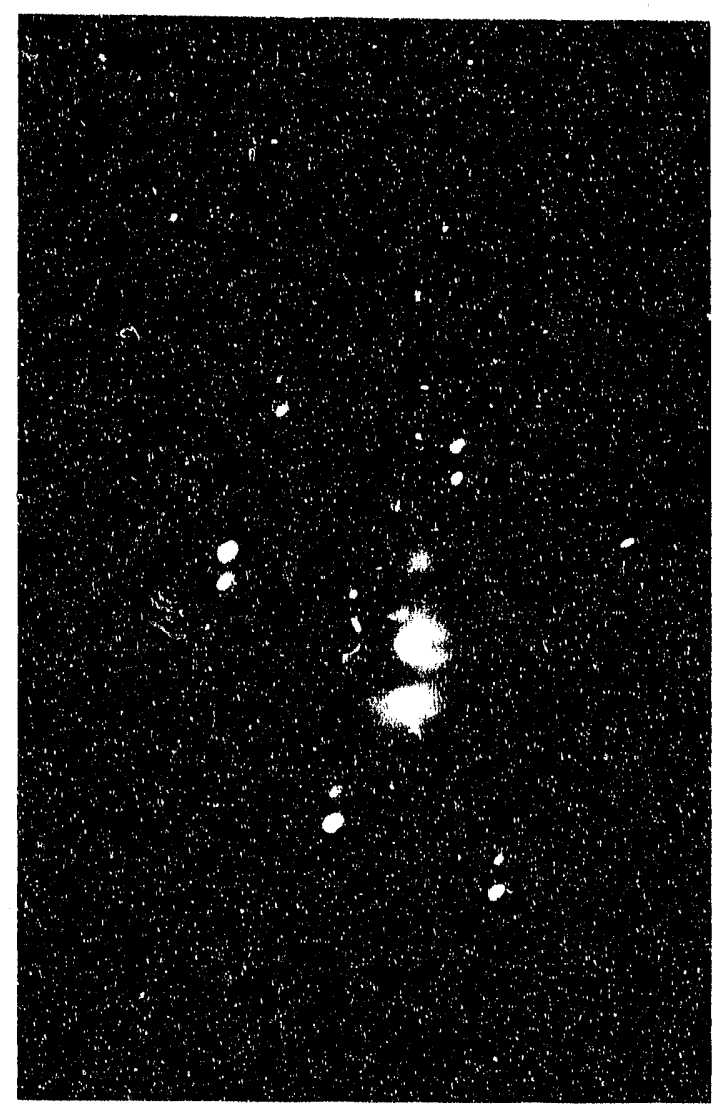

XBB 910-8014A

Figure 5.8: Diffraction pattern of $\operatorname{Mo}(28,4,1)-(1 \times 1)$ S surface. 


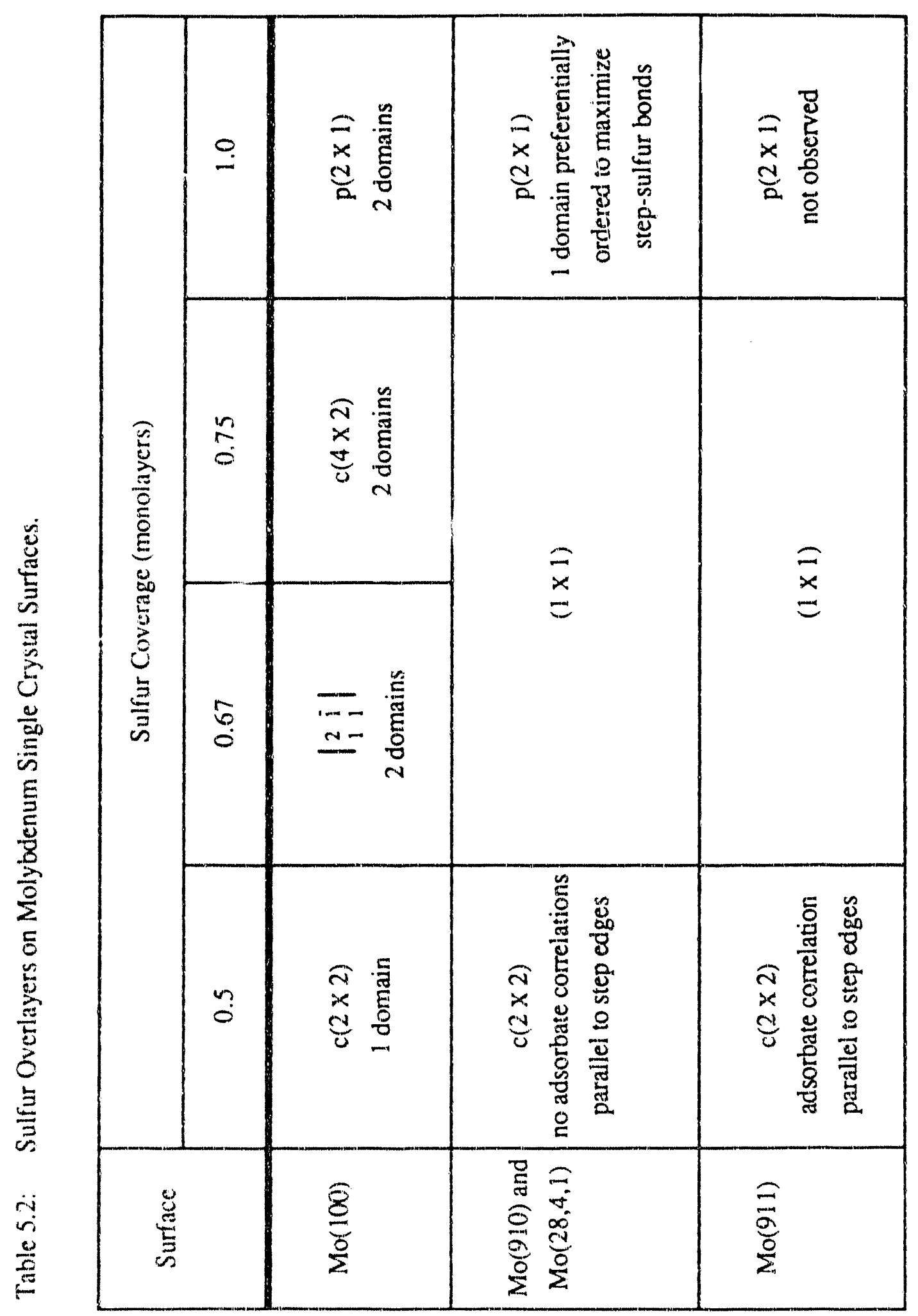


We have found that on the sulfur covered surfaces, double height steps and kinks are present. This is different from results reported on other vicinal body centered cubic (100) surfaces. Dowben el al..$^{5}$ found that nitrogen induced a double step to single step transition on vicinal $\mathrm{Fe}(100)$ surfaces. In the entire coverage range studied $(<0.1-1 \mathrm{ML})$, sulfur induced step-doubling. Our results are thus also different from results of Lanzillotto and Bernasek ${ }^{1}$ on sulfur induced step-doubling of the $\mathrm{Pt}(\mathrm{S})-[6(111) \times(100)]$ surface. While these authors have found that annealing surfaces with low sulfur coverages $(<0.05 \mathrm{ML}$ ) induces step doubling, they report that surface coverages exceeding $0.25 \mathrm{ML}$ leave the platinum surface unreconstructed. A possible explanation for the restructuring observed in this work is that the number of most strongly binding chemisorption sites is increased by such a restructuring.

\subsection{Cobalt Overlayers on Molybdenum Surfaces}

We have determined the structures formed by depositing cobalt overlayers on flat and vicinal $M o(100)$ surfaces. In section 4.2, we have already discussed typical growth modes as well as the factors which determine the particular growth mode of metal overlayers on metal substrates. The deposition of one monolayer of cobalt on the Mo(100) surface at room temperature caused the LEED pattern to deteriorate significantly. After annealing at $870 \mathrm{~K}$ for one minute, a much shapper $(1 \times 1)$ diffraction pattern was observed. The diffraction spots of this $(1 \times 1)$ pattern however were not as sharp as those observed for the clean $\mathrm{Mo}(100)$ surface. These resuits indicate that the first cobalt monolayer grows psuedomorphically on the $\mathrm{Mo}(100)$ surface. Since the diffraction pattern of the cobalt covered surface was not as sharp as that of the clean Mo(100) surface, we know that perfect epitaxial growth continuing the bulk periodicity in the surface plane of the 
substrate was not realized.

When multilayers were deposited and annealed, a diffuse diffraction pattern was observed. This result is consistent with a Stranski-Krastanov growth mode. Considering the lattice mismatch between cobalt and molybdenum this growth mode is not surprising. Molybdenum has a body centered cubic structure and the two dimensional lattice constant of the $(100)$ surface is $3.14 \AA$. Cobalt has hexagonal closed packed and face centered cubic structures $^{18}$. There are no hexagonal closed packed or face centered cubic surfaces with square symmetry that would easily allow one to envision a square cobalt unit cell on the square $\mathrm{Mo}(100)$ surface. However since the lattice constant of the fcc (111) surface of cobalt is $2.51 \AA$ and the lattice constant of the hcp (0001) surface is $2.52 \AA$, a large lattice mismatch can be expected between cobalt overlayers and the Mo(100) surface.

He and Goodman have determined the superstructures formed by deposition and annealing of cobalt overlayers on the $\mathrm{Mo}(110)$ surface $^{19}$. According to their work, annealing cobalt coverages less than three-quarters of a monolayer at $400 \mathrm{~K}$ results in pseudomorphic growth. At higher cobalt coverages and various annealing temperatures, $(9 \times 2)$, hexagonal and $(2 \times 2)$ superstructures are seen. The $(9 \times 2)$ and hexagonal structures are believed to correspond to a distorted $\mathrm{Co}(0001)$ lattice and three dimensional cobalt clusters on Mo(110) respectively. In agreement with what we observe for the growth of cobalt on $\mathrm{Mo}(100)$, these workers have found that only submonolayers grow pseudomorphically on $\mathrm{Mo}(110)$.

The deposition and subsequent annealing at $870 \mathrm{~K}$ of one monolayer of cobalt on the $\mathrm{Mo}(910)$ and $(28,4,1)$ surfaces results in the observation of a sharp ( $3 \times 1)$ diffraction pattern (figure 5.9). It is interesting that a superstructure exists on these stepped surfaces which does not exist on the flat (100) surface. This is different from the behavior of sulfur overlayers on the vicinal surfaces, in that in this case only a subset of the ordered structures formed on the flat surfaces nucleate on the vicinal surfaces. Similar to the $p(2 \times 1) S$ 
(a)

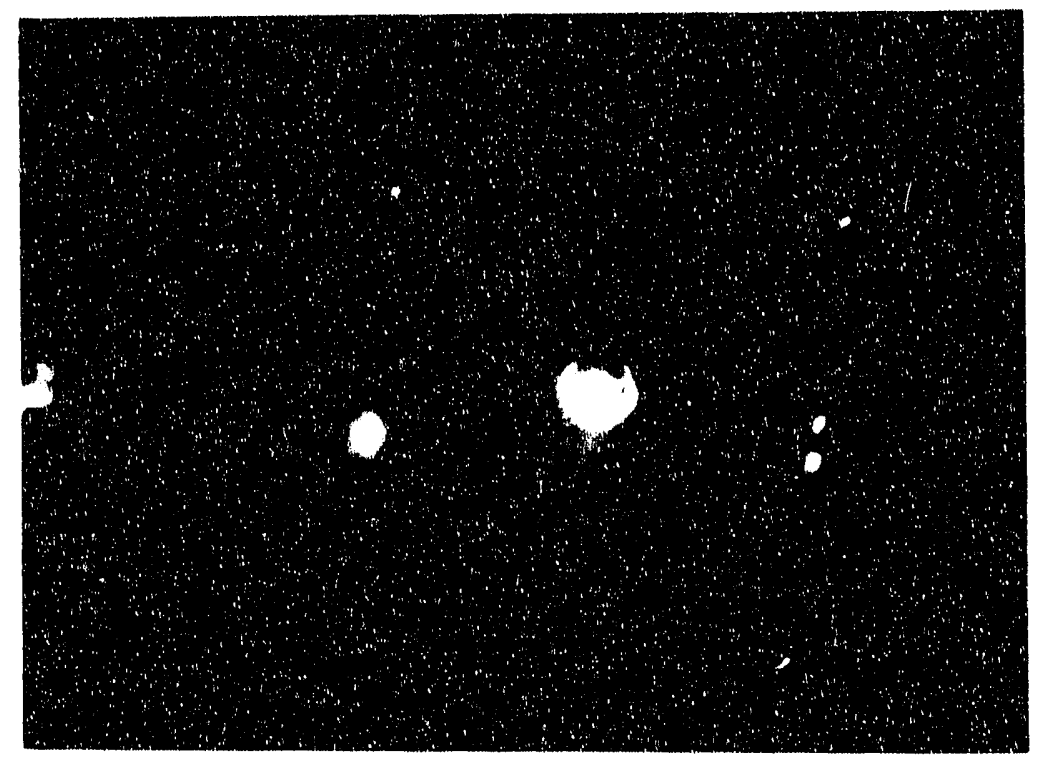

$70 \mathrm{eV}$

(b)

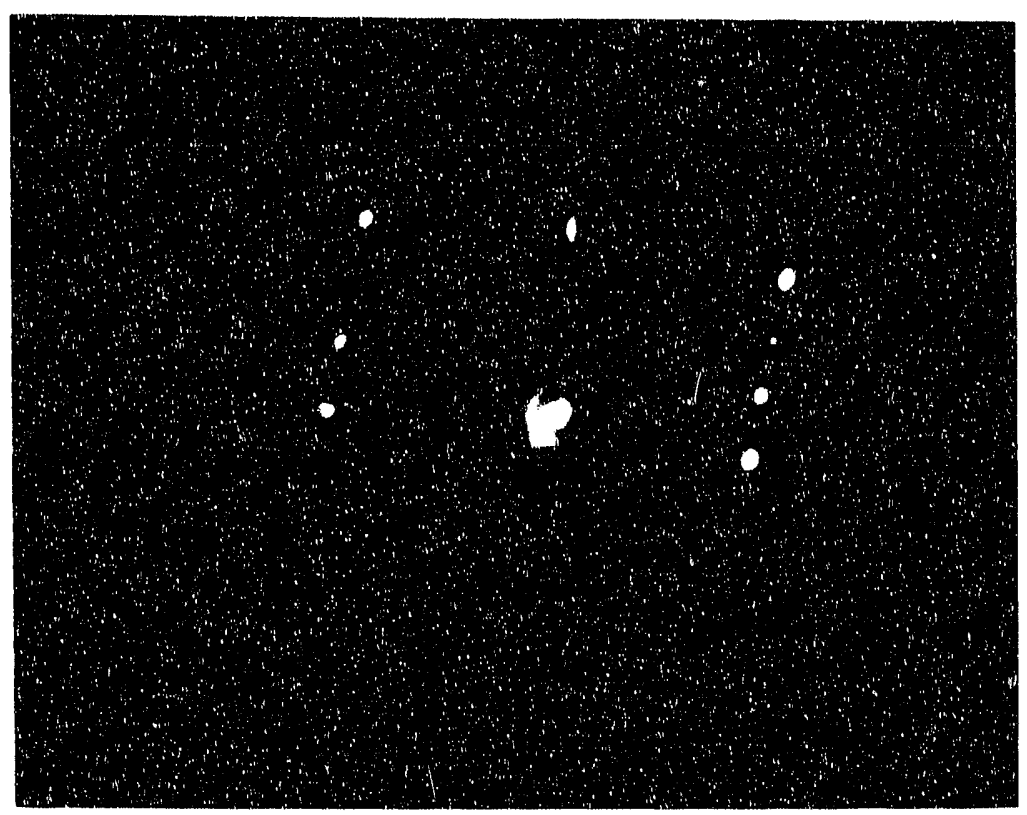

$59 \mathrm{eV}$

CBB 890-10702A

Figure 5.9: Diffraction patterns of (a) clean $M o(910)$ and (b) $M o(910)-(3 \times 1) C o$ surfaces showing that only the domains with the longer side of the unit cell (eriple periodicity axis) perpendicular to the step edges are formed. 
overlayer, the $(3 \times 1)$ Co overlayer is formed on the $\operatorname{Mo}(910)$ and $(28,4,1)$ surfaces but not on the $\mathrm{Mo}(911)$ surface. Furthermore, in agreement with what is observed for the $\mathrm{p}(2 \times 1) \mathrm{S}$ overlayers on vicinal surfaces (section 5.3$)$, for the $(3 \times 1)$ Co superstructure, only the domains with the triple periodicity axis perpendicular to the step edge are ordered. This result again shows that the presence of steps, and in particular different step directions, can dictate which superstructures and in particular which domain of a given superstructure are formed on the terraces. For all of the surfaces, the deposition and subsequent annealing of cobalt multilayers caused the observed diffraction pattern to deteriorate. On the vicinal surfares, the intensity of the $(3 \times 1)$ beams were barely visible at a coverage of approximately three monolayers. This behavior is not surprising in view of the results discussed in chapter 4 that suggest that three dimensional growth occurs at coverages greater than one monolayer.

\subsection{Cobalt Overlayers on Sulfur Covered Molybdenum Surfaces}

Since the goal of this thesis is to model cobalt promoted molybdenum sulfide catalysts, it is relevant to look at coadsorbed sulfur and cobalt overlayers on molybdenum surfaces. Additionally, by coadsorbing cobalt and sulfur, we have the opportunity to evaluate the effect of sulfur on the growth mode of cobalt overlayers on molybdenum surfaces. It has been shown by Tromp and coworkers that for semiconductor interfaces, the introduction of surface active species (surfactants) modify the growth mode without significant levels of incorporation ${ }^{20}$. For example, a preadsorbed monolayer of arsenic changes the growth mode for germanium on $\mathrm{Si}(100)$ from a Stranski-Krastanov growth mode to a layer-by-layer growth mode. For a surfactant to function effectively, it must be 
sufficiently mobile to avoid incorporation at a given growth rate, ie. it must segregate to the surface. We have shown in the previous chapter that sulfur segregates to the top of cobalt overlayers.

Cobalt was deposited on the Mo(100) $-c(2 \times 2) S$ surface. This superstructure corresponds to a sulfur coverage of $0.5 \mathrm{ML}$. After cobalt deposition, the surfaces were annealed at $870 \mathrm{~K}$ for one minute. From figure 5.10 , it can be seen that the overlayer kept this $\mathrm{c}(2 \times 2)$ symmetry after evaporation of about four monolayers of cobalt. With increasing cobalt coverage, the background intensity increased somewhat, but the spots still remained fairly sharp. The fact that ordered structures are seen after multilayers of cobalt are deposited on this sulfur covered molybdenum surface suggests that the underlying cobalt overlayer is relatively well ordered. This contrasts with the results from the clean Mo(100) surface in which case cobalt multilayers clearly formed three dimensional clusters. In this system, sulfur acts as a surfactant increasing the number of cobalt monolayers that grow in a quasi layer by layer fashion $\left(1<\theta_{\mathrm{Co}_{0}}<\sim 4 \mathrm{ML}\right)$. Because of the large lattice mismatch between cobalt and molybdenum, extensive layer-by-layer growth over several monolayers is not expected even in the presence of a surfactant. It will be interesting to evaluate the effectiveness of sulfur and other surfactants such as nitrogen and oxygen for the growth of metal overlayers on metal substrates in cases where the lattice mismatch of the system is small $(<2 \%)$.

The adsorption of cobalt on $M o(100)-c(4 \times 2) S$ and $M o(100)-p(2 \times 1) S$ surfaces was also studied ${ }^{21}$. After one monolayer of cobalt was evaporated and annealed at $870 \mathrm{~K}$ on these sulfur covered surfaces, $(3 \times 1)$ superstructures were seen in both cases. Again, this $(3 \times 1)$ structure was seen for cobalt coverages up to four monolayers. This result again suggests that sulfur improves the quasi layer-by-layer growth of this system. The diffraction patterns corresponding to these surfaces are shown in figures 5.11 and 5.12 . With increasing coverage, the background intensity increases indicating a decreasing 
(a)

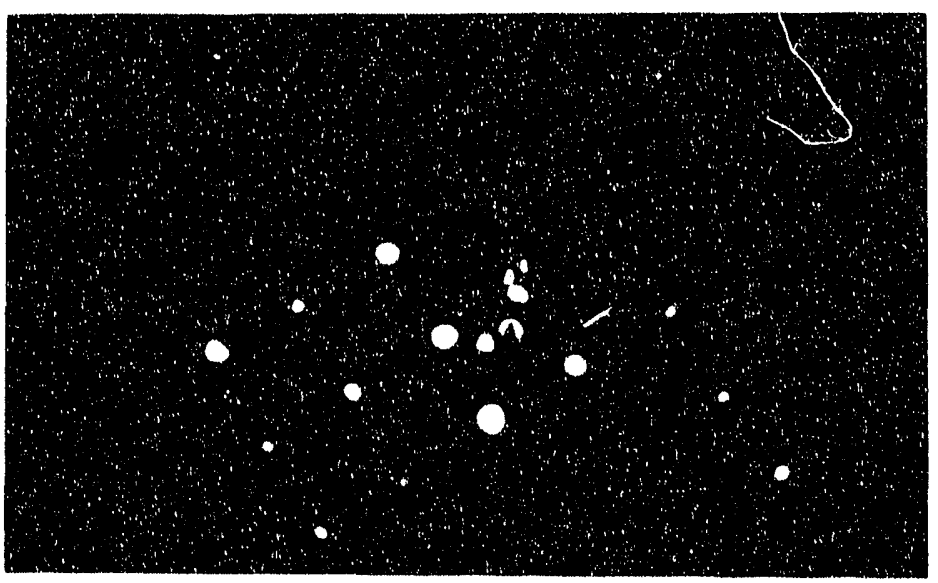

(b)

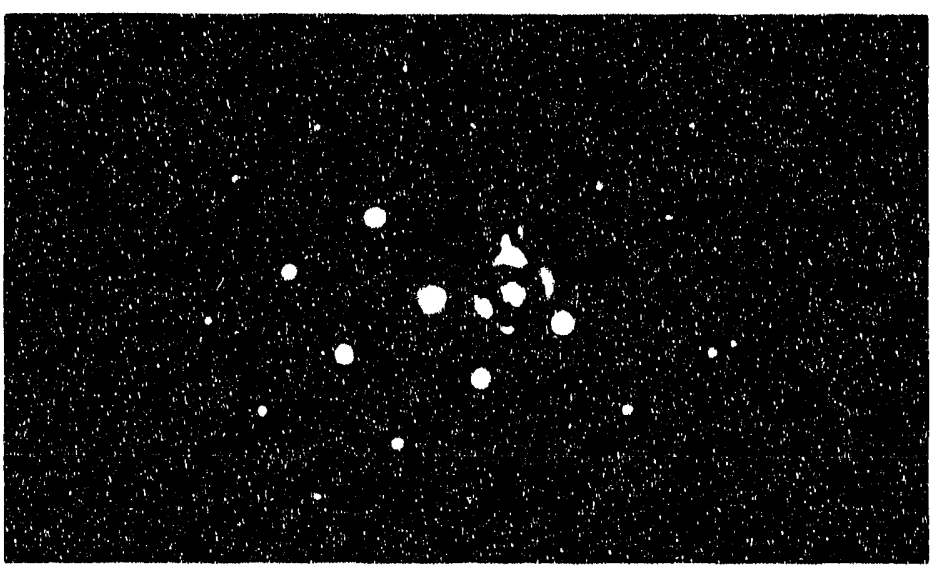

$140 \mathrm{eV}$

(c)

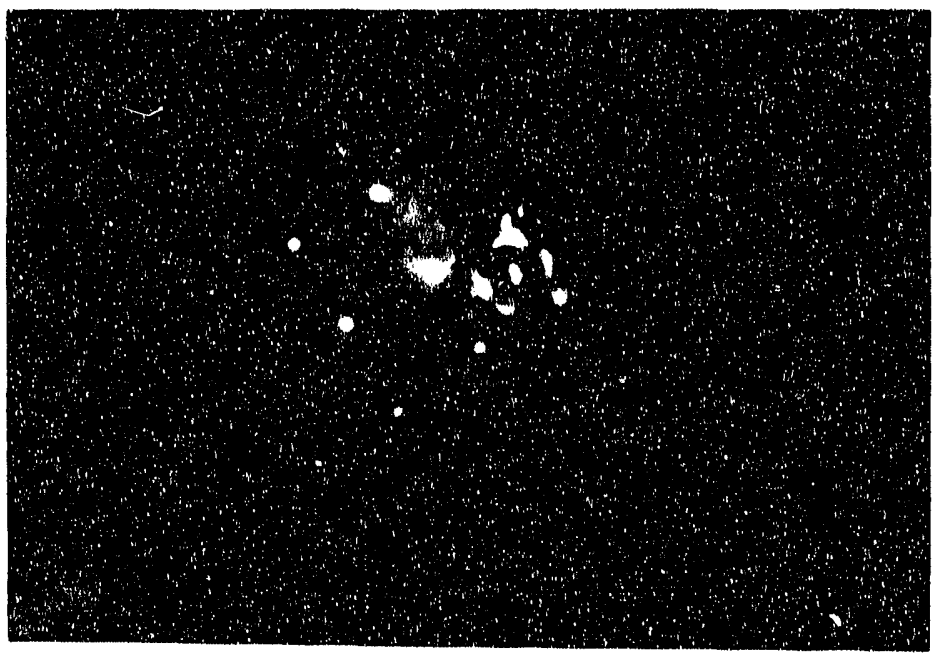

$140 \mathrm{eV}$

CBB 890-10704A

Figure 5.10: Diffaction patterns of cobalt overlayers on the Mo(10)( ) - $c(2 \times 2) \mathrm{S}$ surface, (a) $\theta_{(b)}=() \mathrm{ML}$, (b) $\theta_{\left(c_{1}\right)} \sim 1 \mathrm{ML}$, and (c) $\theta_{(b)} \sim 4 \mathrm{ML}$. 
(a)

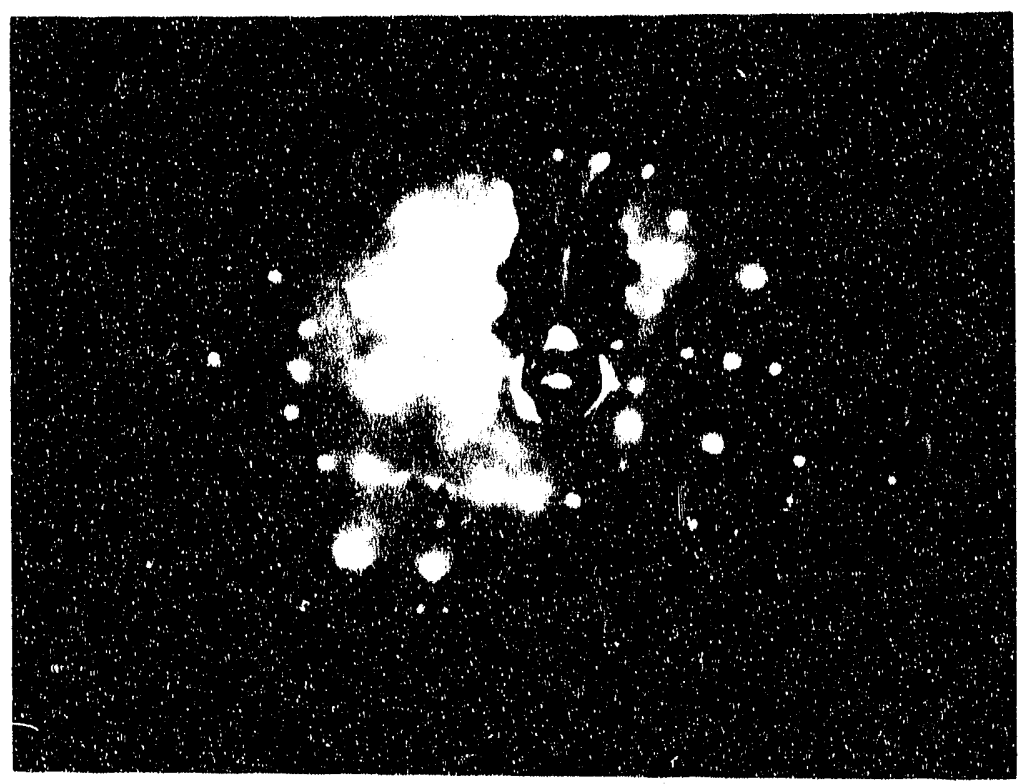

$120 \mathrm{eV}$

(b)

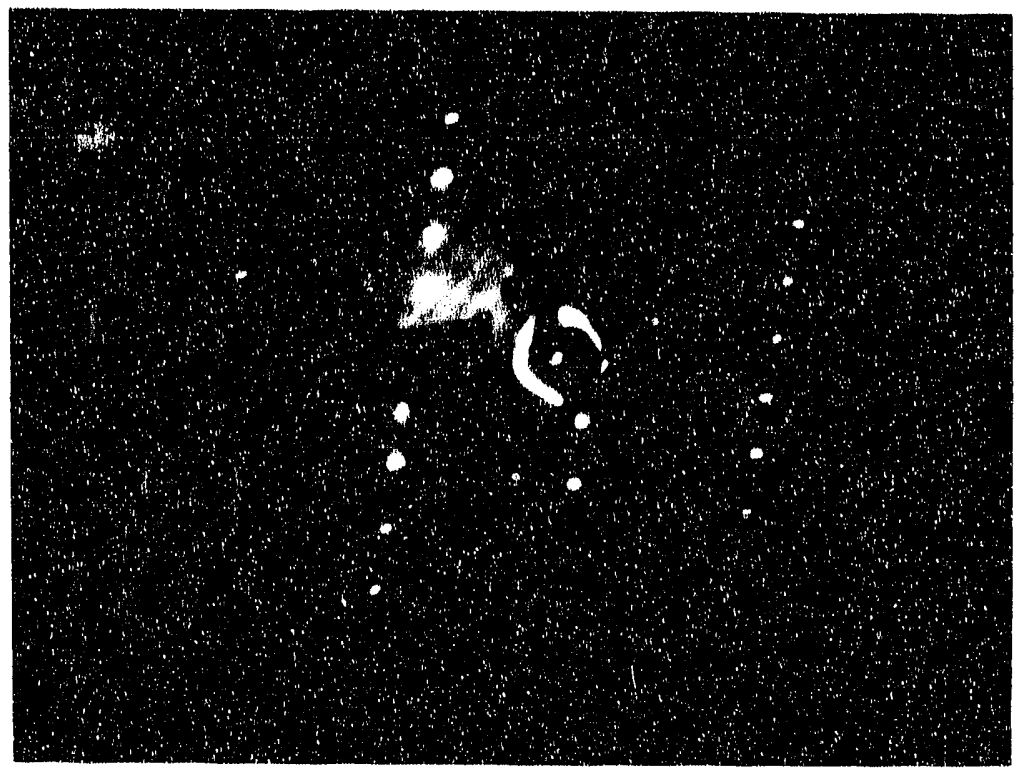

$100 \mathrm{eV}$

CBB 890-10705A

Figure 5.11: Diffraction patterns of cobalt overlayers on the $\operatorname{Mo}(1(0))-c(4 \times 2) S$ surface, (a) $\theta_{C_{0}}=0 \mathrm{ML}$ and (b) $\theta_{C_{0}}-1 \mathrm{ML}$. 
(a)

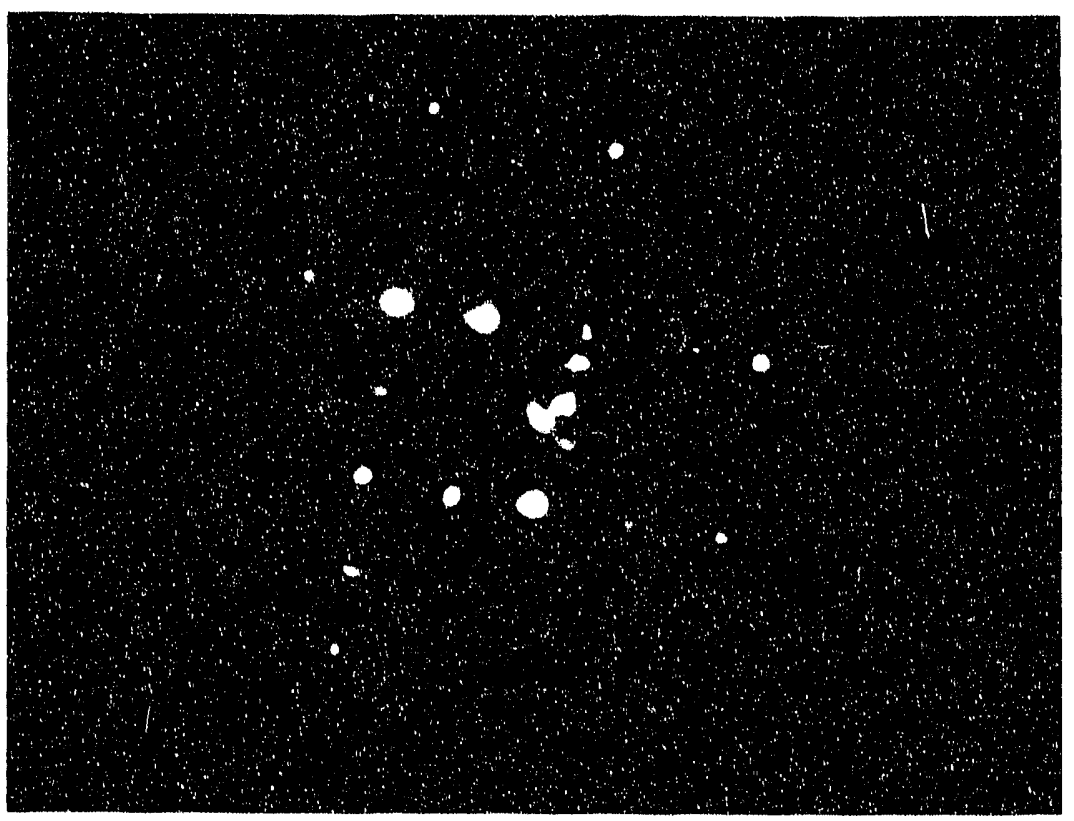

$115 \mathrm{eV}$

(b)

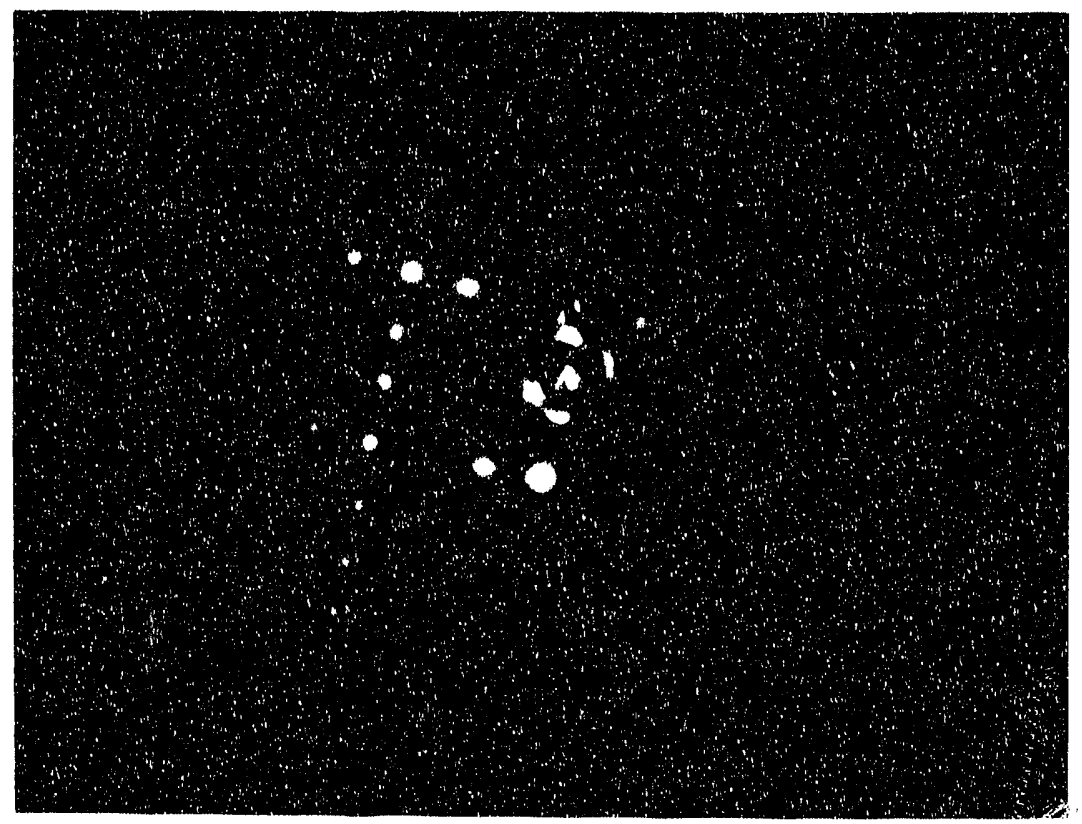

Figure 5.12: Diffraction patterns of cobalt overlayers on the $\mathrm{Mo}(100)-\mathrm{p}(2 \times 1) \mathrm{S}$ surface, (a) $\theta_{\mathrm{Co}}=0 \mathrm{ML}$ and (b) $\theta_{\mathrm{Cu}} \sim 1 \mathrm{ML}$. 
quality of the films. We have not determined the relationship between the $(3 \times 1)$ Co structure formed on the vicinal surfaces (section 5.4) and those formed on the flat $M o(100)-c(4 \times 2) S$ and $M o(100)-p(2 \times 1) S$ surfaces.

The $(3 \times 1)$ structure was seen when one monolayer of cobalt was deposited on a $\mathrm{Mo}(100)$ surface covered with at least $0.75 \mathrm{ML}$ of an ordered sulfur layer. When less than one monolayer of cobalt was deposited and annealed on these higher sulfur covered surfaces, only diffuse $(1 \times 1)$ diffraction patterns were observed. When cobalt was deposited first and the surface annealed at $870 \mathrm{~K}$ to order the cobalt, and then sulfur was deposited, again only ( $1 \times 1)$ spots in a diffuse background were observed. A study of the effect of annealing temperature on this structure showed that the $(3 \times 1)$ spots started to appear dimly after annealing at approximately $720 \mathrm{~K}$. Using an annealing temperature of $770 \mathrm{~K}$, the $(3 \times 1)$ diffraction beams became clearly visible and by $870 \mathrm{~K}$, the annealing temperature generally used, the beams were very sharp. Annealing at or above $940 \mathrm{~K}$, caused the disappearance of these beams and the appearance of diffuse intensity. On the vicinal $\mathrm{Mo}(910)$ and $\mathrm{Mo}(28,4,1)$ surfaces, deposition of cobalt on the $\mathrm{p}(2 \times 1) \mathrm{S}$ structure also resulted in the formation of a ( $3 \times 1)$ structure. This $(3 \times 1)$ structure was ordered only in the same domain as the $\mathrm{p}(2 \times 1) \mathrm{S}$ structure. The ordered structures formed by cobalt deposition on the clean and sulfur covered surfaces are summarized in table 5.3.

\subsection{Summary}

In this chapter, we have reported examples of sulfur induced step-doubling on stepped and kinked molybdenum surfaces. Not only can adsorbates restructure vicinal surfaces, but the presence of steps and kinks on these surfaces play a role in determining which ordered overlayers form on the terraces. We have also demonstrated that the number 
Table 5.3: Ordered Structures formed by Cobalt Deposition on Clean and Sulfur Covered Molybdenum Surfaces.

\begin{tabular}{|c|c|}
\hline Initial Surface & $\begin{array}{r}\text { Ordered Structures Formed } \\
\text { after Cobalt Deposition }\end{array}$ \\
\hline clean $\mathrm{Mo}(100)$ & $(1 \times 1)$ \\
clean $M o(910)$ and $M o(28,4,1)$ & $(3 \times 1) 1$ domain \\
clean $M o(911)$ & $c(2 \times 2)$ \\
$M o(100)-c(2 \times 2) S$ & $(3 \times 1) 2$ domains \\
$M o(100)-c(4 \times 2) S$ & $(3 \times 1) 2$ domains \\
$M o(100)-p(2 \times 1) S$ & $(3 \times 1) 1$ domain \\
$M o(910)-p(2 \times 1) S$ and $M o(28,4,1)-p(2 \times 1) S$ & \\
\hline
\end{tabular}


and type of ordered sulfur and cobalt structures that form on flat and vicinal surfaces are different and furthermore depend on the step direction. While in the case of sulfur, only a subset of the ordered overlayers formed on the flat surface nucleate on the vicinal surfaces, in the case of cobalt an ordered structure that does not form on the flat surface is formed on two of the vicinal surfaces.

On vicinal surfaces, domains of adsorbate islands are interrupted by the presence of steps. In many cases this results in random translational and rotational relationships between adsorbate islands on different terraces. Nucleation at step edges can facilitate adsorbate correlations perpendicular to step edges and result in preferential orientation of certain domains. This was shown for the $\mathrm{p}(2 \times 1) \mathrm{S}$ and $(3 \times 1)$ Co overlayers on Mo(910) and $\operatorname{Mo}(28,4,1)$ surfaces. Additionally, as shown for the $M o(911)-c(2 \times 2) S$ surface, certain step directions can cause positional correlations parallel to step edges between adsorbate islands on different terraces. These effects of steps can potentially have far reaching consequences. It is at least in principle possible to grow overlayers which would otherwise form more than one equivalent domain on flat surfaces in which one large domain is preferentially ordered and correlated by a fortunate pairing of the chemisorption system and step direction.

\section{References}

[1] A-M. Lanzillotto and S. L. Bernasek, J. Chem. Phys. 843553 (1986).

[2] M. Sokolowski and H. Pfnür, Phys. Rev. Lett. 63183 (1989).

[3] M. Sokolowski and H. Pfnür, unpublished work.

[4] G. Comsa, G. Mechtersheimer a.1d B. Poelsema, Surf. Sci. 119159 (1982);

G. Comsa, G. Mechtersheimer and B. Poelsema, Surf. Sci. 119172 (1982). 
[5] P. A. Dowben, M. Grunze and R. G. Jones, Surf. Sci. 109 L519 (1981).

[6] T.-M. Lu, L.-H. Zhao, M. G. Lagally, G.-C. Wang and J. E. Houston, Surf. Sci. 122519 (1982).

[7] D. G. Kelly, Ph.D. Thesis, University of California, Berkeley, (1987).

[8] V. Maurice, L. Peralta, Y. Berthier and J. Oudar, Surf. Sci. 148623 (1984).

[9] L. J. Clarke, Surf. Sci. 91131 (1980).

[10] D. G. Kelly, R. F. Lin, M. A. Van Hove and G. A. Somorjai, Surf. Sci. 22497 (1989).

[11] P. Rous, D. Jentz, D. G. Kelly, R. Q. Hwang, M. A. Van Hove and G. A. Somorjai to be published in the proceeding ICSOS-3, Milwaukee, WI, July 1990.

[12] B. Marchon, P. Bernhardt, M. E. Bussell, G. A. Somorjai, M. Salmeron and W. Siekhaus, Phys. Rev. Lett. 601166 (1988).

[13] D. Jentz, private communication.

[14] X. W. Wang and S. G. Louie, Surf. Sci. 226257 (1990).

[15] Jim Dumphy, private communication.

[16] C. C. Knight and G. A. Somorjai, accepted in Surf. Sci. (1992).

[17] Strictly speaking, the clean unrestructured surfaces have the same periodicity as the restructured surfaces with two atom height steps. This periodicity, 9-fold and 7-fold perpendicular to the step edge of the $\operatorname{Mo}(910)$ and $\operatorname{Mo}(28,4,1)$ surfaces respectively, arises from the fact that consecutive terraces are shifted by half an atomic distance relative to each other on the unrestructured surfaces. This can be seen in figure 3.6. However, calculations in the kinematic approximation show that for the unrestructured surfaces with monoatomic steps, the intensities of the $n / 7$ and $n / 9$ order spots with odd $n$ are very low.

[18] J. M. MacLaren, J. B. Pendry, P. J. Rous, D. K. Saldin, G. A. Somorjai, M. A. Van Hove and D. D. Vvedensky, Surface Crystallographic 
Information.Service: A Handbook of Surface Structures, D. Reidel

Publishing Company, Dordrecht, Holland.

[19] J. -W. He and D. W. Goodman, Surf, Sci. 24529 (1991).

[20] M. Copel, M. C. Reuter, M. Horn von Hoengen and R. M. Tromp, Phys. Rev. B 4211682 (1990); F. K. LeGoues, M. Copel and R. M. Tromp, Phys. Rev B 42, 11690 (1990).

[21] C. C. Knight and G. A. Somorjai, Surf. Sci. 240101 (1990). 


\section{Chapter 6 Thiophene Hydrodesulfurization Over Molybdenum Surfaces}

\subsection{Introduction}

As discussed in chapter 1, one of the motivations for this work is to gain insight into the promoter role of cobalt for molybdenum sulfide hydrodesulfurization (HDS) catalysts. We have attempted to do this by studying the chemisorption and reactivity of cobalt overlayers on model rnolybdenum surfaces. In the previous three chapters, we have examined the growth and structure of cobalt and sulfur monolayers on flat, stepped and kinked molybdenum surfaces. In chapter 5, we showed that the ordered cobalt and sulfur domains are preferentially arranged to maximize adsorbate-step interactions on viciral molybdenum surfaces. In this chapter, we use these surfaces to catalyze the hydrodesulfurization of thiophene. In particular, we aro interested in examining the effect of cobalt on the thiophene HDS rate and product distribution. Sections 2.2.2 and 2.5 outlined how catalytic reactions are performed using the in situ atmospheric pressure cell incorporated into the ultrahigh vacuum chamber. We end the chapter with a summary of the work performed.

Following the exposure of $\mathrm{Mo}(100)$ surfaces to typical reaction gas mixtures of about 3 Torr of thiophene and 780 Torr of hydrogen at $613 \mathrm{~K}$, both carbon $\left(\theta_{C} \sim 0.8 \mathrm{ML}\right)$ and sulfur $\left(\theta_{S} \sim 0.2 \mathrm{ML}\right)$ deposits are left on the metal surface ${ }^{1}$. Radiotracer ${ }^{14} \mathrm{C}$ and ${ }^{35} \mathrm{~S}$ studies show that both of these overlayers are stable on $\mathrm{Mo}(100)$ surfaces under typical 
reaction conditions ${ }^{2,3}$. Indeed the rate of removal of ${ }^{14} \mathrm{C}$ and ${ }^{35} \mathrm{~S}$ was found to be about two orders of magnitude lower than the thiophene HDS rate. Because of the stability of these overlayers, it seems likely that carbon and sulfur deposits play significant roles in the surface chemistry of molybdenum surfaces. Previous work in our group has examined the effect of preadsorbed carbon and sulfur overlayers on thiophene HDS over Mo(100) surfaces. The reaction rates are quoted per unit surface area of the initially clean metal surface. They are not normalized to account for the fact that when adsorbates are deposited on molybdenum surfaces, the exposed molybdenum area available for catalysis decreases. Bussell et al. ${ }^{2}$ found that identical reaction rates were observed over carbon covered surfaces independent of the initial carbon coverage $\left(0<\theta_{C}(\mathrm{ML})<1\right)$. On the other hand, preadsorbed sulfur had a site blocking effect. At a sulfur coverage of about $0.67 \mathrm{ML}$, the measured rate of thiophene HDS was about half that of the initially clean surface ${ }^{3}$. The different effect of carbon and sulfur was attributed to the different sizes of these two adsorbates. Unrelated work by Benziger and Madix ${ }^{4}$ and MacLaren et al. ${ }^{5}$ have suggested that the ability of an adsorbate to poison a catalytic surface is related to the height that it extends above the metal surface. In light of these results, Bussell et al. ${ }^{2}$ suggested that while the smaller size of carbon (atomic radius $=0.77 \AA$ ) allows it to sit in fourfold hollow sites without extending above the molybdenum surface, the larger size of sulfur (atomic radius $=1.04 \AA$ ), causes this adsorbate to extend significantly above the surface molybdenum atoms.

The deactivation of the molybdenum surface after thiophene conversions of about $5 \%$ was attributed to accumulation of $\mathrm{H}_{2} \mathrm{~S}$ in the batch reactor ${ }^{2}$. Examination of the surfaces after evacuation of the reactant gases showed that the surfaces were covered with almost one monolayer of both carbon and sulfur, irrespective of the initial surface composition. Since sulfur preadsorbed in vacuum lowered the observed reactivity while initially clean Mo(100) surfaces maintained their higher activity, it was suggested that this 
additional sulfur was deposited on the surfaces during evacuation of the high pressure cell. It is possible that sulfur is not deposited on the surface during the reaction because of the high coverage of adsorbed hydrogen. Alternatively, if sulfur was deposited during the reaction, it was only weakly bound to the surface. Furthermore, it was suggested that the key feature of thiophene HDS over molybdenum surfaces is that the active site is a molybdenum-carbide site, which selectively breaks carbon-sulfur bonds unlike the initially clean surface that indiscriminately breaks both carbon -carbon and carbon-sulfur bonds ${ }^{1}$.

\subsection{HDS Over Flat and Vicinal Molybdenum Surfaces}

A diagram of the various thiophene HDS reaction paths is shown in figure 1.2. Thiophene HDS reactions were carried out under the same conditions described previously ( $\mathrm{P}_{\mathrm{Th}}=3$ Torr, $\mathrm{P}_{\mathrm{H}_{2}}=780$ Torr). The molybdenum crystals were heated to $613 \mathrm{~K}$, except when the temperature was varied to determine the temperature dependence of the reaction rate. The HDS rate over molybdenum surfaces was determined by measuring the initial rates of product formation using gas chromatography. Because the reaction only proceeds to about $5 \%$ conversion before the onset of deactivation, it is not possible to accurateiy measure the decrease in the thiophene concentration. A typical gas chromatograph of the reaction mixture was shown in figure 2.18 . From such a chromatograph, it can be seen that butenes, butadiene and butane comprise over $95 \%$ of the reaction products. $C_{1}-C_{3}$ products are only produced in minor amounts $(<5 \%)$. Of the $\mathrm{C}_{4}$ products, 1 -butene is formed most abundantly even though table 1.2 shows that butane is the thermodynamically favored product. The product distribution is thus governed by kinetic constraints. Typical product accumulation curves for thiophene $\operatorname{HDS}$ over $M O(28,4,1)$ surfaces are shown in 


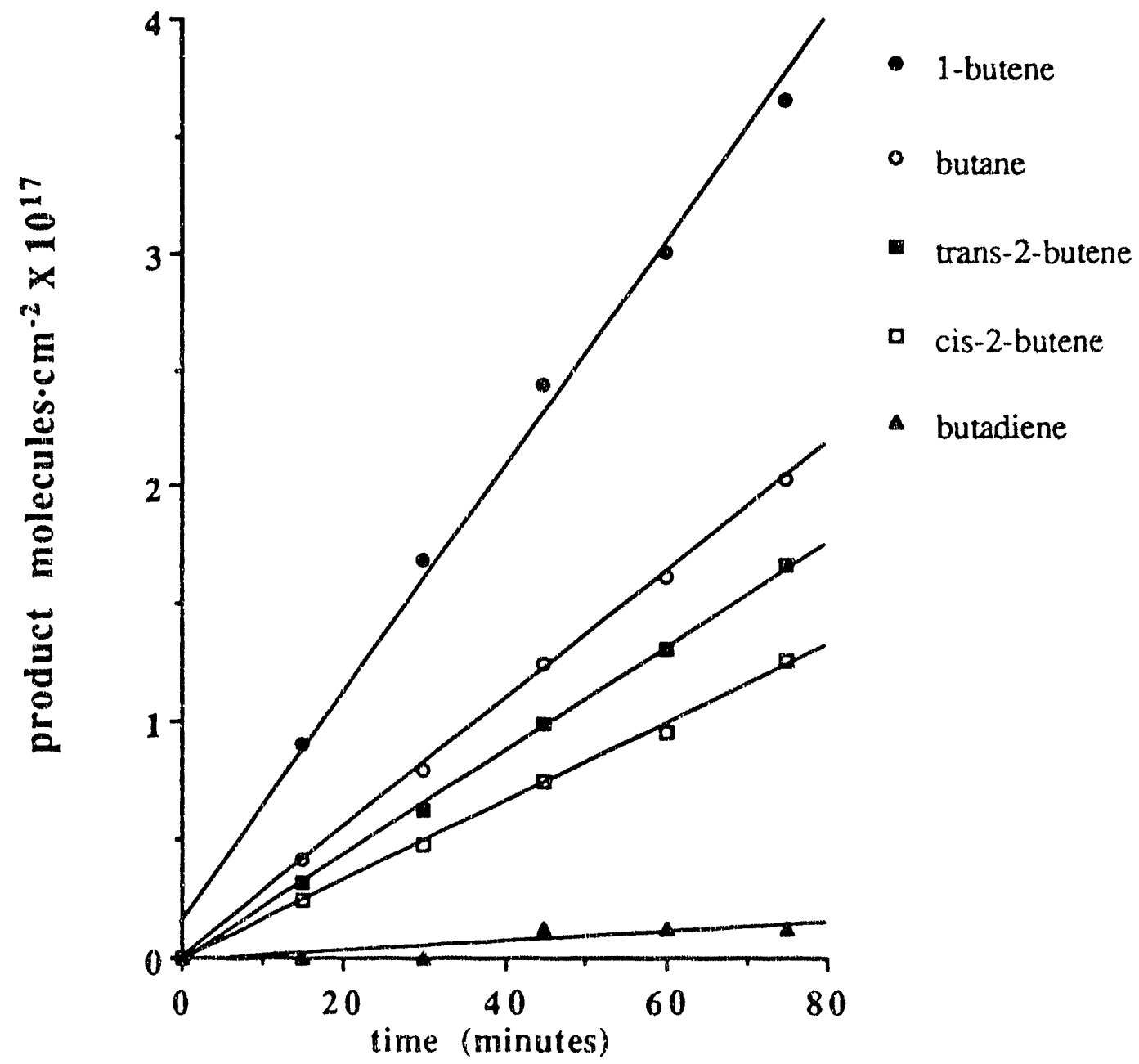

Figure 6.1: Product accumulation curves for thiophene HDS over Mo(910) surfaces. $\mathrm{P}_{\mathrm{Th}} \sim 3$ Torr, $\mathrm{P}_{\mathrm{H}_{2}} \sim 780$ Torr, $\mathrm{T}=613 \mathrm{~K}$. 
figure 6.1.

Gellman $^{6}$ compared the rate of thiophene HDS over the low Miller index (100), (110) and (111) and high Miller index (910) surfaces. It was found that the total initial rate of products formed over the low index surfaces was about $1.1 \times 10^{14}$ molecules $\cdot \mathrm{s}^{-1} \mathrm{~cm}^{-2}$ at $613 \mathrm{~K}$ and a pressure of about 3 Torr of thiophene and 780 Torr of hydrogen. The reaction rate over the $\mathrm{Mo}(910)$ surface was about $50 \%$ higher. In this work, we have measured the thiophene HDS rate over the (100), $(910),(911)$ and $(28,4,1)$ surfaces of molybdenum. The average rates that we have measured over the (100) and (910) surfaces are about $1.5 \times 10^{14}$ and $2.4 \times 10^{14}$ product molecules $\cdot \mathrm{s}^{-1} \cdot \mathrm{cm}^{2}$ using the same conditions. These numbers are in qualitative agreement with previous results. These rates along with those observed over the $(911)$ and $(28,4,1)$ surfaces are compared in figure 6.2 . In general, the vicinal surfaces have reaction rates between 50 and $100 \%$ higher than the flat (100) surface, with the kinked surface having the highest rate $\left(\sim 2.7 \times 10^{14}\right.$ product molecules $\cdot \mathrm{s}^{-1} \cdot \mathrm{cm}^{-2}$ ). An Arrhenius plot of the temperature dependence of the total HDS reactivity in the temperature range of $553 \mathrm{~K}$ to $613 \mathrm{~K}$ over the Mo(910) surface is shown in figure 6.3. An activation energy of about $10 \mathrm{kcal} \cdot \mathrm{mol}^{-1}$ was calculated.

One of the problems encountered was that the observed reaction rate over the same crystal often varied when the crystal was remounted. Occasionally, the rates varied by as much as a factor of two. As described in section 2.2 .1 , the molybdenum samples are spotwelded between rhenium wires and $\mathrm{Pt} / \mathrm{Pt} 10 \% \mathrm{Rh}$ thermocouples are spotwelded to the top edge of the crystals. Periodically these connections to the crystal broke and had to be respotwelded. The different reaction rates measured were attributed to different and uncontrollable heating characteristics of the sample, ie. inhomogeneities in heating that could not be visually detected while heating in UHV. 


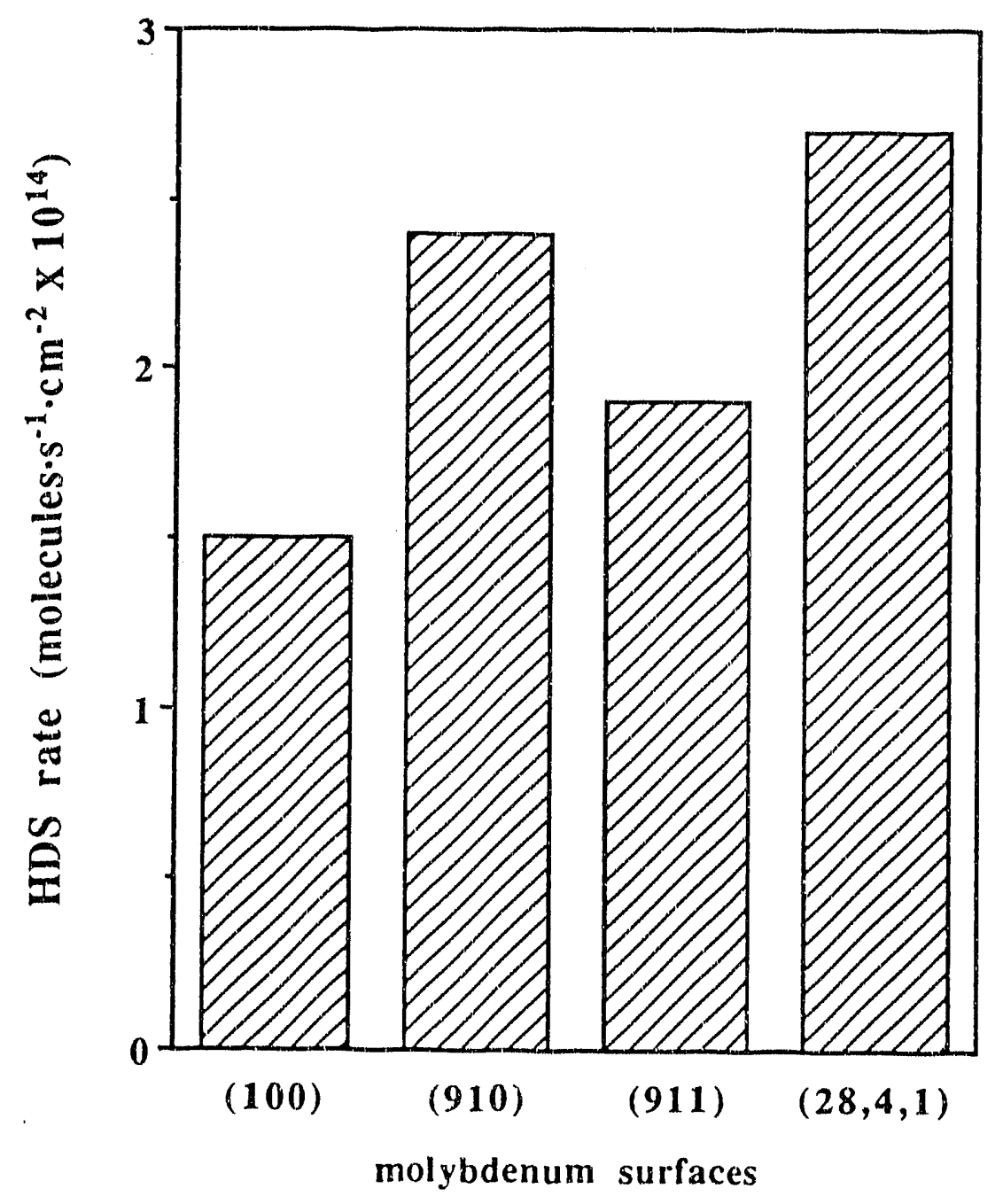

Figure 6.2: Initial thiophene HDS rates over $\mathrm{Mo}(100), \mathrm{Mo}(910), \mathrm{Mo}(911)$ and Mo(28,4,1) surfaces. $\mathrm{P}_{\mathrm{Th}} \sim 3$ Torr, $\mathrm{P}_{\mathrm{H}_{2}} \sim 780$ Torr, $\mathrm{T}=613 \mathrm{~K}$. 


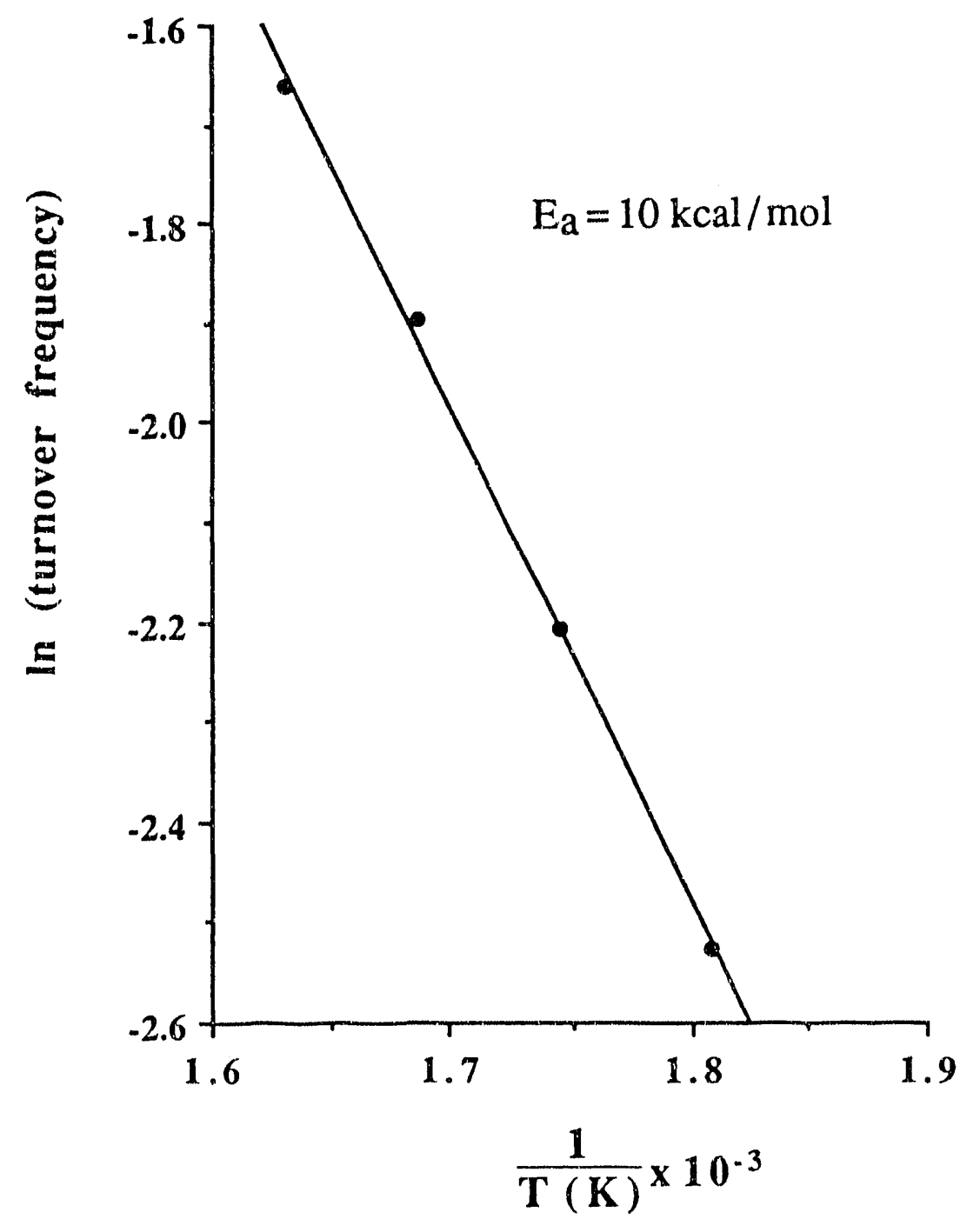

Figure 6.3: Temperature dependence of thiophene HDS over Mo(910) surfaces, $\mathrm{P}_{\mathrm{Th}} \sim 3$ Torr, $\mathrm{P}_{\mathrm{H}_{2}} \sim 780$ Torr. 


\subsection{Thiophene HDS Over Sulfur and Carbon Covered Molybdenum Surfaces}

The effect of sulfur and carbon overlayers on flat and vicinal Mo(100) surfaces was investigated. Sulfur overlayers were prepared and characterized as described in sections 2.2.4 and 5.3. Carbon was deposited by exposing the surfaces to ethylene at $800 \mathrm{~K}$. Exposures typically varied between $5 \times 10^{-9}$ and $1 \times 10^{-8}$ Torr for $30-120 \mathrm{~s}$ depending on the carbon coverage desired. The reactions were carried out at $613 \mathrm{~K}$ using a gas mixture of $\sim 3$ Torr of thiophene and 780 Torr of hydrogen. As found on the flat $\mathrm{Mo}(100)$ surface, preadsorbed sulfur overlayers decreased the reactivity of the vicinal $\mathrm{Mo}(100)$ surfaces.

While clean $\operatorname{Mo}(28,4,1)$ surfaces have reaction rates of about $2.7 \times 10^{14}$ molecules $\cdot \mathrm{s}^{-1} \cdot \mathrm{cm}^{-2}$, the chemisorption of one monolayer of sulfur causes the reaction rate to decrease to about $1.7 \times 10^{14}$ molecules $\cdot \mathrm{s}^{-1} \cdot \mathrm{cm}^{-2}$. This relative decrease in the reaction rate is comparable to the decrease seen on $\mathrm{Mo}(100)$ surfaces with the chemisorption of sulfur. On the other hand, differences were found in the effect of carbon on flat and vicinal Mo(100) surfaces. The observed reaction rate over $M o(100)$ surfaces was found to be independent of the carbon coverage ${ }^{2}$. In this work, we have found that preadsorbed carbon overlayers decrease the rate relative to the clean surface. The carbon Auger lineshapes observed in this work (figure 2.10 (b)) as well as previous work ${ }^{2}$ show that the carbon overlayer is carbidic in nature. Figure 6.4 shows a plot of the thiophene HDS rate as a function of the carbon coverage on the $\operatorname{Mo}(28,4,1)$ surfaces. Although the reason for the different effect of carbon overlayers on flat and vicinal surfaces is not clear, it might be attributed to carbon poisoning of the molybdenum step atoms. It is possible that while these step atoms are responsible for the higher rates measured over vicinal surfaces they are also more susceptible to poisoning by carbon.

The product distribution over the sulfur and carbon covered surfaces was not 


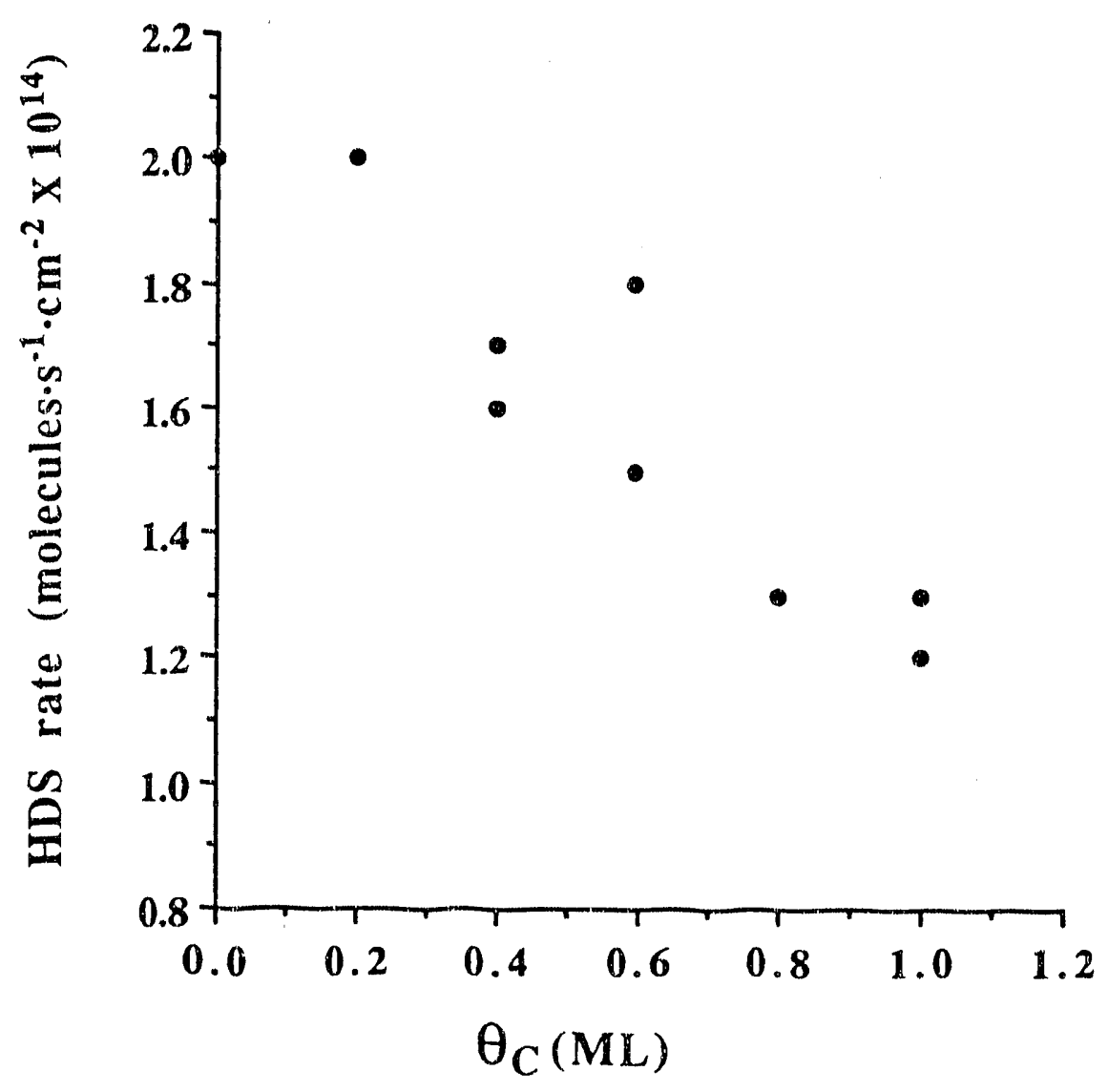

Figure 6.4: Thiophene HDS rate as a function of the initial carbon coverage on $\operatorname{Mo}(28,4,1)$ surfaces. The rates are quoted per unit surface area of the initially clean $\mathrm{Mo}(28,4,1)$ surface. $\mathrm{P}_{\mathrm{Th}} \sim 3$ Torr, $\mathrm{P}_{\mathrm{H}_{2}} \sim 780 \mathrm{Torr}, \mathrm{T}=613 \mathrm{~K}$. 
meaningfully changed relative to the clean surface. The extent of the reaction varied between about 3 and $5 \%$ conversion. At these conversion levels, we found that the selectivity was independent of the extent of the reaction. From figure 6.1 , it can be seen that the product distribution does not change significantly over the seventy-five minute period that the reaction is run. On all of the surfaces, 1-butene is the major product followed by butane, trans-2-butene, cis-2-butene and butadiene (figure 2.18). This result is different from results of previous reports ${ }^{1,6,7}$ in which butane was produced in lesser quantities than trans and cis-2-butene. The actual chromatographs of the reaction mixture of these works confirming that good separation of the products and especially 1-butene and butane was achieved are not available for comparison. Although the absolute product composition varied between different experiments, there were no systematic changes in the product distribution that could be attributed to the adsorbate composition or coverage.

\subsection{Effect of Cobalt on Thiophene HDS over Molybdenum Surfaces}

Cobalt was deposited on the molybdenum surfaces using the cobalt source described in section 2.2.5. Coverages were calibrated using the Auger uptake curve shown in figure 4.2. Figures $6.5-6.7$ show the effect of cobalt overlayers on the reaction rate of thiophene HDS over the $\mathrm{Mo}(100), \mathrm{Mo}(910)$ and $\mathrm{Mo}(28,4,1)$ surfaces. In the case of the $\operatorname{Mo}(100)$ and $\operatorname{Mo}(28,4,1)$ surfaces, a clear decrease in the reaction rate is observed with increasing cobalt coverage. Since cobalt foils have a lower HDS reactivity than molybdenum foils ${ }^{8}$, the decrease in the observed rate can be simply attributed to the blocking of the more active molybdenum sites by cobalt. Cobalt overlayers caused similar decreases in the reaction rate over surfaces with preadsorbed sulfur and carbon layers. 


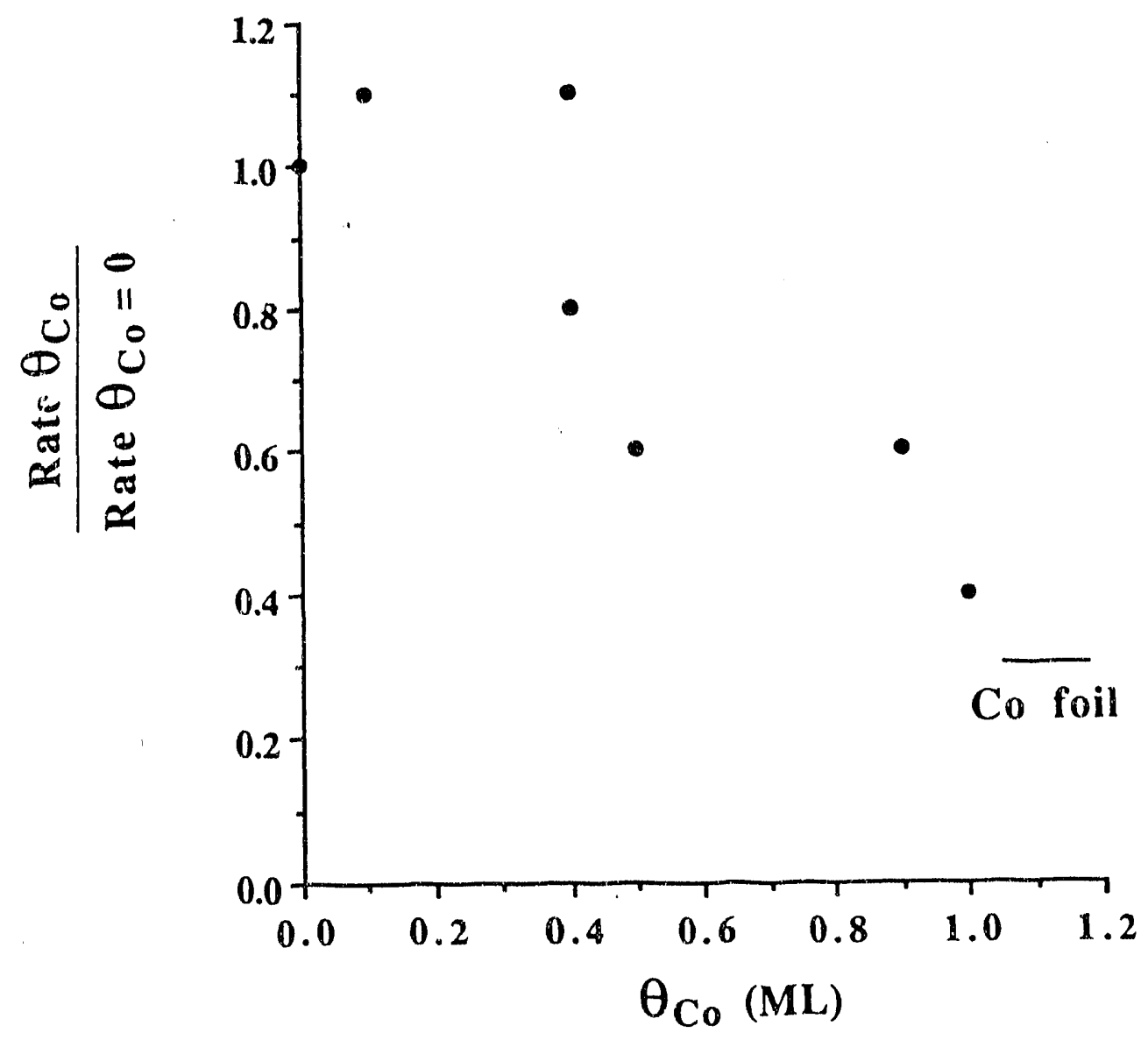

Figure 6.5: Effect of cobalt on the thiophene HDS rate over Mo(100) surfaces. The observed rates are divided by the rate of the initially clean surface. They are not normalized to account for the fraction of the surface that is cobalt covered. The data point for the cobalt foil was taken from reference 8 . $\mathrm{P}_{\mathrm{Th}} \sim 3$ Torr, $\mathrm{P}_{\mathrm{H}_{2}} \sim 780$ Torr, $\mathrm{T}=613 \mathrm{~K}$. 


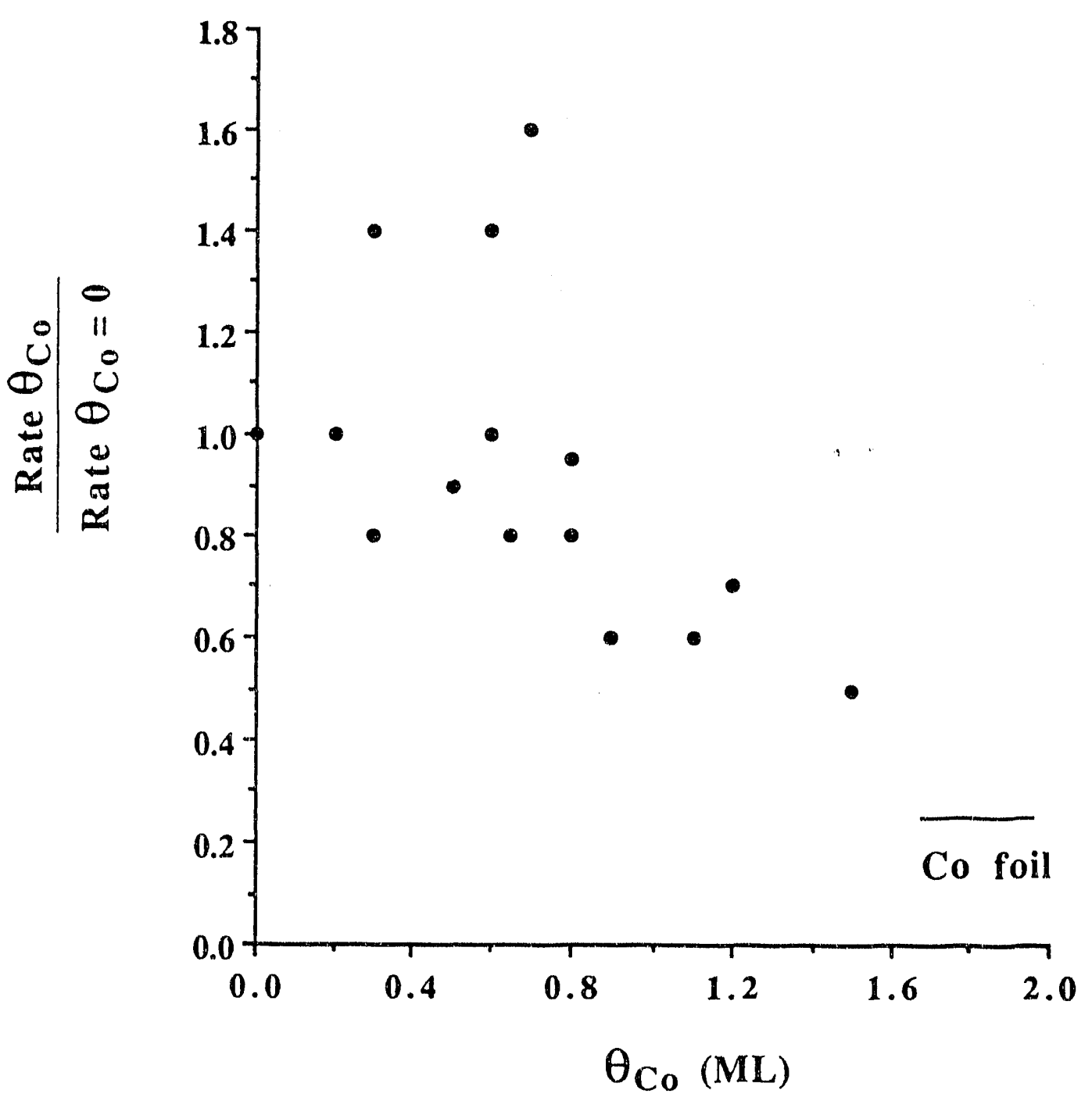

Figure 6.6: Effect of cobalt on the thiophene HDS rate over Mo(910) surfaces. The observed rates are divided by the rate of the initially clean surface. They are not normalized to account for the fraction of the surface that is cobalt covered. The data point for the cobalt foil was taken from reference 8 . $\mathrm{P}_{\mathrm{Th}} \sim 3$ Torr, $\mathrm{P}_{\mathrm{H}_{2}} \sim 780$ Torr, $\mathrm{T}=613 \mathrm{~K}$. 


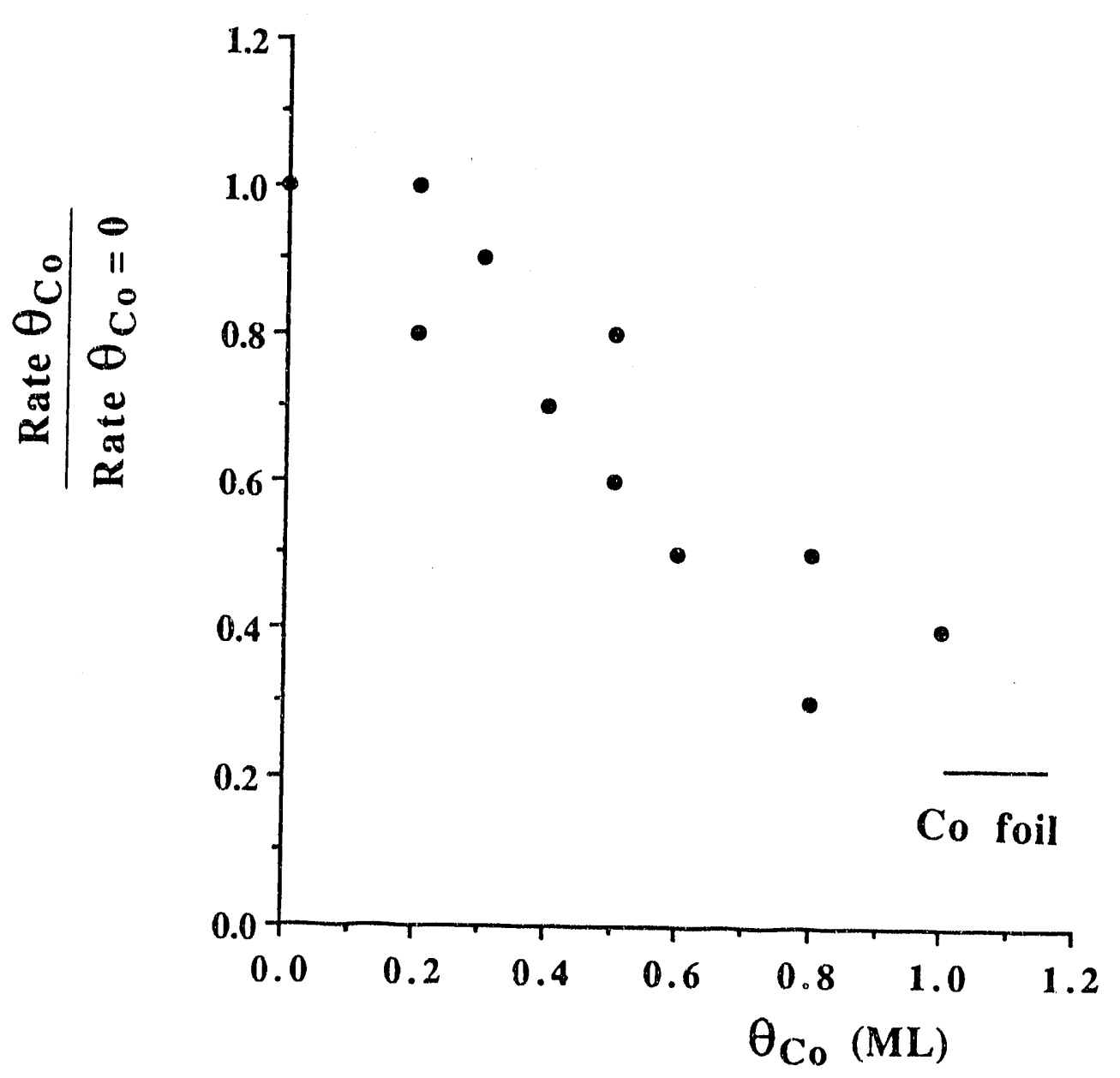

Figure 6.7: Effect of cobalt on the thiophene HDS rate over $\operatorname{Mo}(28,4,1)$ surfaces. The observed rates are divided by the rate of the initially clean surface. They are not normalized to account for the fraction of the surface that is cobalt covered. The data point for the cobalt foil was taken from reference 8 .

$\mathrm{P}_{\mathrm{Th}} \sim 3$ Torr, $\mathrm{P}_{\mathrm{H}_{2}} \sim 780$ Torr, $\mathrm{T}=613 \mathrm{~K}$. 
Clearly, these cobalt overlayers on clean and sulfur covered molybdenum surfaces do not exhibit the promotional effect characteristic of industrial hydrodesulfurization catalysts.

The factors leading to the differences between our model catalytic surfaces and industrial catalysts probably include the differences in sample preparation, reactive phases and operating conditions used. Alumina supported catalysts are prepared by impregnation of $\gamma \mathrm{Al}_{2} \mathrm{O}_{3}$ with aqueous solutions of ammonium heptamolybdate $\left(\left(\mathrm{NH}_{4}\right)_{6} \mathrm{Mo}_{7} \mathrm{O}_{24}\right)$ and cobalt nit ate $\left(\mathrm{Co}\left(\mathrm{NO}_{3}\right)_{2}\right)^{9}$. The catalyst is dried and then calcined at about $800 \mathrm{~K}$ in air to form supported $\mathrm{MoO}_{3}$ and $\mathrm{CoO}$. This precursor is then sulfided, typically in an $\mathrm{H}_{2} \mathrm{~S} / \mathrm{H}_{2}$ atmosphere, to form $\mathrm{MoS}_{2}$ and cobalt sulfides. As discussed in section 1.3, the exact fate of the cobalt is unclear. Not only does the $\mathrm{H}_{2} \mathrm{~S} / \mathrm{H}_{2}$ ratio convert the oxidic precursor to the working sulfide catalysts, but this ratio can also be used to control the sulfur defect concentration.

A key to producing a properly working catalysts is to start with the correct oxide precursor. $\mathrm{MoS}_{2}$ catalysts are prepared by sulfiding $\mathrm{MoO}_{3}$, not $\mathrm{MoO}_{2}$ or molybdenum metal. It seems likely that the preparation of these optimum sulfide catalysts is intimately related to the chemistry and reduction of $\mathrm{Mo}^{+6}$. Furthermore the chemistry occurring on the industrial catalyst is probably characteristic of molybdenum sulfide with the metal in the +4 oxidation state. From studies with electron spin resonance (ESR) spectroscopy, Konings et al. have reported that on cobalt promoted catalysts there is a reduction of $\mathrm{Mo}^{+4}$ to $\mathrm{Mo}^{+3}$ and that the activity of the promoted catalysts is correlated to the intensity of the $\mathrm{Mo}^{+3}$ ESR signal ${ }^{10}$. In either event, the promotional effect of cobalt is not associated with zero valent cobalt or molybdenum. This is borne out by the lack of a promotional effect seen in this work. 


\subsection{Summary}

A surface science approach to catalysis is based on the premise that knowledge of atomic composition and structure gained in UHV environments can be correlated with macroscopic parameters measured under catalytic conditions. In cases where the steady state composition of the surface is low under the reaction conditions, such correlations are possible. However, in the thiophene HDS reaction, the molybdenum surfaces become covered with adsorbate layers. This makes correlations between the initial surface composition and the observed reaction rates difficult.

In the previous two chapters, we have characterized the cobalt and sulfur overlayers formed on flat and vicinal molybdenum surfaces in ultra high vacuum. We have shown that sulfur induces doubling of the step height and that both cobalt and sulfur appear to nucleate at the step edges, thus preferentially ordering into domains that maximize adsorbate-step interactions. However once these surfaces characterized in an UHV environment are exposed to an atmosphere of reactant gases, their surface structure and composition become complicated and neither are they yet characterized. We could not determine the steady state surface composition, if double or single height steps were present, or even if the adsorbaies were still preferentially adsorbed at the step edges. At the end of the reaction, all of the surfaces were covered with sulfur and carbon and their diffraction patterns showed that they were disordered.

Industrially, the addition of cobalt to $\mathrm{MoS}_{2}$ catalysts inc ases the HDS rate of the catalyst by a factor of $10-30^{11}$. We have not found that deposition of cobalt overlayers on clean, sulfur or carbon coverod moly bdenum surfaces leads to an enhanced reactivity for thiophene HDS. On the contrary, a decrease in the reaction rate is seen with increasing cobalt coverage. The lack of enilancement suggests that this promotion is not associated writh metaii: cobait or molythenum. 


\section{References}

[1] M. E. Bussell, Ph.D. thesis, University of California, Berkeley (1988).

[2] M. E. Bussell and G. A. Sornorjai, J. Catal. 10693 (1987).

[3] A. J. Gellman, M. E. Bussell and G. A. Somorjai, J. Catal. 107103 (1987).

[4] J. B. Benziger and R. J. Madix, Surf. Sci. 94119 (1980).

[5] J. M. MacLaren, J. P. Pendry and R. W. Joyner, S 'rf. Sci. 165 L80 (1986).

[6] A. J. Gellman, Ph. D. thesis, University of California, Berkeley (1985).

[7] C. C. Knight, when I used the old integrator also used by M. E. B. and possibly A. J. G. , I also detected lower butane concentrations. As this integrator only provided peak areas without the actual chromatographs, it is not clear how the peak areas were calculated. The present integrator prints out chromatographs with tick marks separating the peaks. It is possible that with the old integrator, the area under closely spaced peaks was improperly calculated.

[8] M. E. Bussell and G. A. Somorjai, Cat. Lett. 31 (1989).

[9] R. Prins, V. H. J. deBeer and G. A. Somorjai, Catal. Rev. Sci. Eng. 311 (1989).

[10] A. J. A. Konings, A. Valster, V. H. J. deBeer and R. Prins, J. Catal. 76466 (1982).

[11] S. Harris and R. R. Chianelli, J. Catal. 9817 (1986). 

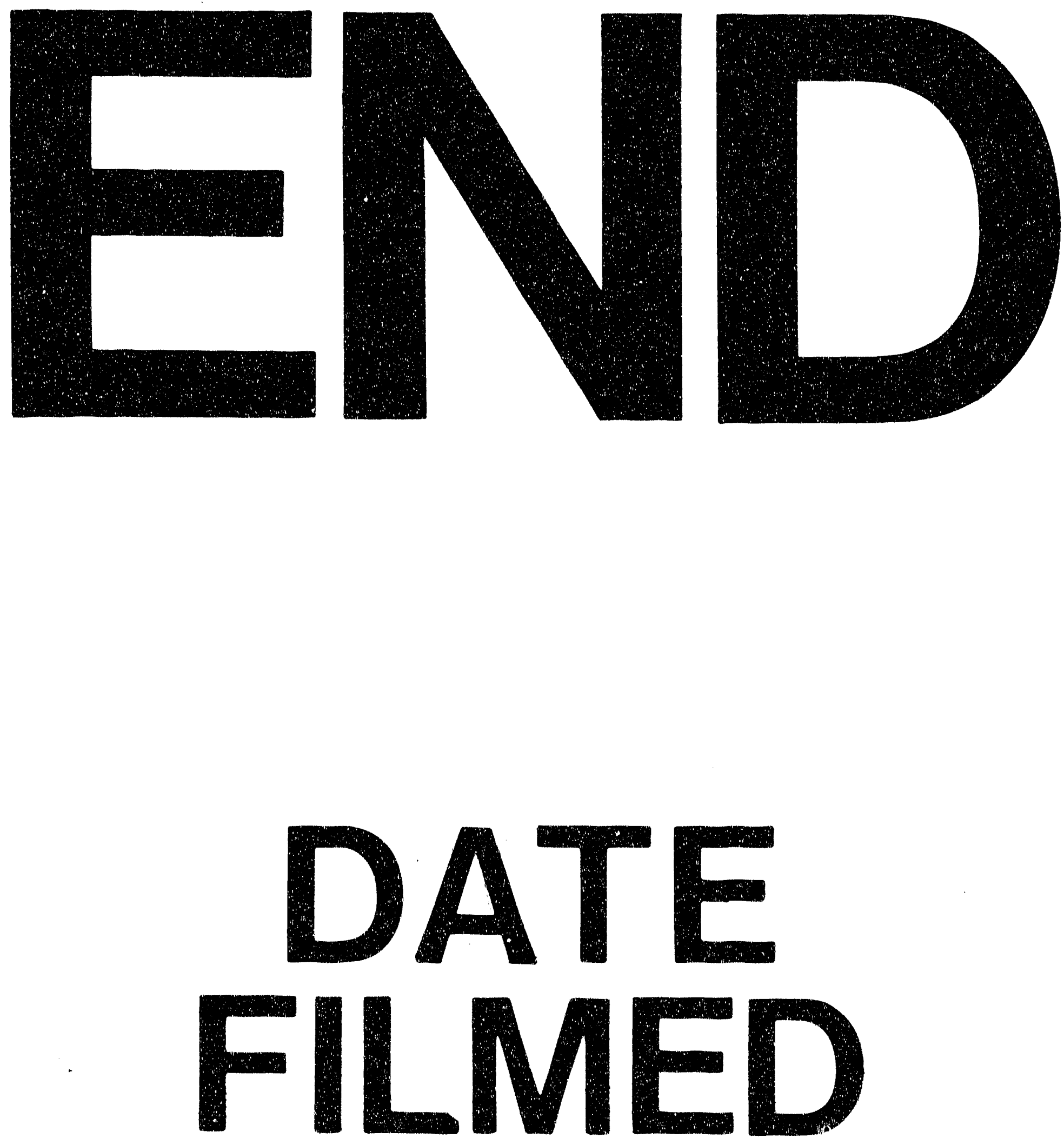

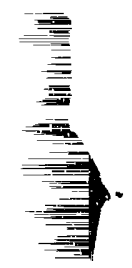

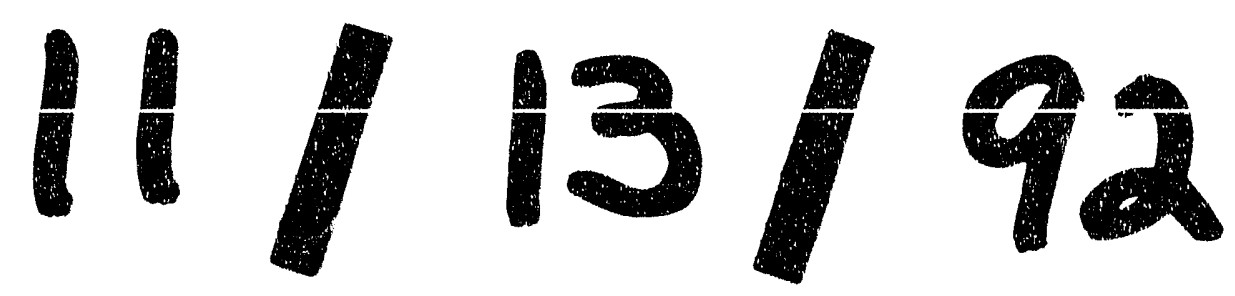


